German Primate Center (DPZ)

Dept. Behavioral Ecology \& Sociobiology/ Anthropology

Determinants of population structure in the world's smallest primate, Microcebus berthae, across its global range in Menabe Central, Western Madagascar

Thesis in the PhD program "Biological Diversity and Ecology" for the degree "Doctor rerum naturalium" (Dr. rer. nat.)

Livia Schäffler

Submitted on September 29th, 2011 
University of Göttingen - Centre for Biodiversity and Ecology (GCBE)

\section{Disputation committee}

Prof. Dr. Peter M. Kappeler, German Primate Center

Prof. Dr. Matthias Schäfer, J.F.Blumenbach Institute of Zoology and Anthropology Prof. Prof. h.c., Dr. Michael Mühlenberg, Centre for Nature Conservation Prof. Dr. Joachim Saborowski, Büsgen Institute of Forest Sciences and Forest Ecology Prof. Dr. Hans-Rolf Gregorius, Büsgen Institute of Forest Sciences and Forest Ecology Prof. Dr. Stefan Scheu, J.F.Blumenbach Institute of Zoology and Anthropology 


\title{
Determinants of population structure in the world's smallest primate, Microcebus berthae, across its global range in Menabe Central, Western Madagascar
}

\begin{abstract}
This study investigated the population ecology of the world's smallest primate, Microcebus berthae, which is locally endemic to Menabe Central, the largest remaining tract of dry deciduous forest in Western Madagascar. Mme. Berthae's mouse lemur is a highly charismatic and therefore a promising flagship species for the protection of this dry forest remnant. The ecological specialist regionally co-occurs with a closely related generalist, the more widely distributed and abundant Microcebus murinus, which is competitively superior to $M$. berthae. Interspecific coexistence cannot be explained by ecological differentiation, as the congeners considerably overlap in feeding niches and microhabitat utilization. The mouse lemurs co-occur with several other members of the family Cheirogaleidae (Cheirogaleus medius, Mirza coquereli, and Phaner pallescens). We examined the ecological structure within this species assemblage across $M$. berthae's global range in order to identify determinants of $M$. berthae's distribution. The spatial distribution and abundance of cheirogaleids was assessed by distance sampling and trapping on various spatial scales and analyzed on the population as well as on the individual level.
\end{abstract}

Results show that $M$. berthae's population is largely limited to pristine habitats and sensitive to human frequentation. Interspecific competition between the mouse lemurs was indicated on the population level, and led to negative interspecific associations in degraded habitat and to habitat partitioning along anthropogenic disturbance gradients during the scarce dry season. In nondegraded habitat, interactions with third agents regulated interspecific competition of Microcebus spp. to a level stabilizing their coexistence. Mirza coquereli represents an intraguild predator of Microcebus spp., which exerts predation pressure disproportionally on M. murinus. Consistent with this notion, $M$. coquereli negatively affected $M$. murinus populations, but was positively associated with $M$. berthae on a regional scale. The species' interspecific distribution across spatial and temporal heterogeneities affirmed $M$. coquereli's stabilizing impact on the coexistence of mouse lemurs.

In local co-occurrence, intense interspecific competition between mouse lemur individuals was not indicated. Mirza coquereli expelled $M$. murinus individuals from their immediate vicinity only during the dry season, whereas it did not impact the distribution of $M$. berthae individuals. During rainy season activity, $C$. medius operated complementarily on the local scale, as individuals were found in negative association with inferior competitor M. murinus, but positively associated with $M$. berthae individuals.

We conclude that interspecific coexistence of Microcebus spp. is stabilized by a complex agentmediated spatial storage effect. Closely related species create refuges from competition for $M$. berthae in productive habitat, whereas anthropogenic environments provide an escape from intraguild predation and exclusive resources to $M$. murinus. Persistence of $M$. berthae does therefore not only rely on the maintenance of forested area in Menabe Central, but also on the preservation of habitat content. Extinction thresholds may be exceeded by habitat degradation as it corrupts fundamental interspecific mechanisms or by loss of a keystone species that stabilizes mouse lemur coexistence. 


\section{INTRODUCTION}

\section{The topic of structure in ecological sciences}

Understanding species distribution and abundance, i.e. "ecological structure" has long been a fundamental question in ecological sciences (Stokstad 2009). Ecological theory proposes two main processes to determine the distribution of species within communities, which are defined as assemblages of co-occurring species that potentially interact with one another (sensu Connell \& Slatyer 1977, Cavernder-Bares \& Wilczek 2003). First, environmental filtering constrains the number of species comprising a community within a particular habitat, as the distribution of a species complies with biotic and abiotic habitat factors required to meet ecological demands. Second, intra- as well as interspecific interactions structure communities. Behavioral characteristics, such the social system, determine population structure intraspecifically. Species distributions that could theoretically be realized based on ecological demands, are further positively or negatively affected by interspecific interactions with co-occurring species (Cornwell et al. 2006). In addition to adaptive processes, species distributions might also be determined by historical or chance effects during colonization (Hubbell 1997; Condit et al. 2002).

\section{Environmental filtering}

Environmental characteristics have long been recognized to determine species' distribution and abundance, as well as community composition, at various scales (Condit et al. 2002, Engelbrecht et al. 2007). Physical stressors, such as latitude, altitude, or aridity, predominantly limit the biogeographic distribution of terrestrial species (Brown et al. 1996). On the regional level, spatial variation in abundance roughly reflects differential habitat suitability, as species pursue the distribution of limiting factors that are ecologically required (e.g. along ecological gradients: Pinkus-Rendón et al. 2006, Engelbrecht et al. 2007). Structural characteristics and intrinsic productivity of the habitat locally determine its maximum carrying capacity, which is defined as maximum population density in equilibrium (Verhulst 1838, Wilson and Bossert 1971, both in Seidl \& Tisdell 1999). However, closer examination of the relationship between carrying capacity and population abundance did not reveal simple "tracking" of most productive habitats, and the deviation of population densities from maximum carrying capacity can depend on interactions with co-occurring species (Roughgarden 1974). 
As ecological demands, and consequently species distributions, differ considerably between taxa, various species compositions emerge from divergent habitats. Some authors propose habitat selection on various levels as the major mechanism structuring ecological communities (Lindenmayer et al. 2000). Communities under identical environmental conditions were found to converge in ecologically important traits, reflecting shared ecological tolerances (e.g. Fukami et al. 2005, Cornwell et al. 2006, Engelbrecht et al. 2007, Kraft et al. 2008). As ecological niches tend to be phylogenetically conserved (Swenson et al. 2006, Lessard et al. 2009), habitat filtering may lead to phylogenetic clustering, i.e. coexisting species are more closely related than expected by chance (Cavender-Bares et al. 2004).

\section{Interspecific interactions}

Coexisting species can alter habitat suitability for one another via direct or indirect interactions that alter individual fitness, and thus mutually influence their abundance and spatial distribution. Even though the intensity of interspecific interactions generally increases with rising population density of the interacting species, density-dependent influence on community structure can also be exerted by rare species (Strauss 1991).

\section{Direct interspecific effects: Competition}

Competition (e.g. Tokeshi et al. 1999, Gotelli \& McCabe 2002, Ziv \& Kotler 2003, Sanderson 2004, López-Gómez \& Mollina-Meyer 2006) and predation (Estes \& Palmisano 1974, Caswell 1978, Glasser 1979, Holt 1984, Holt et al. 1994, Holt \& Polis 1997) have been most intensely investigated as interspecific interactions determining species composition within ecological communities (Tokeshi et al. 1999, Stokstad 2009). Several other interactions have the potential to structure communities, including infection, parasitism, co-evolution, mutualism, and mimicry (Sanderson et al. 2009), but, being beyond the scope of this study, they are not elaborated here.

Organisms can escape competition by ecological differentiation on various levels (Amarasekare 2003, Mészena et al. 2006), e.g. by differential resource utilization (resource partitioning: MacArthur \& Levins 1967, Thornton et al. 2004, Fischer \& Gates 2005, Zapata et al. 2005). Alternatively, organisms can escape competition by temporal niche partitioning (behavior: e.g. Leaver \& Daly 2001, Hattori 2002, Leibold et al. 2004; life history: Herrera et 
al. 1996) or some form of spatial separation (spatial habitat partitioning: e.g. Sachot et al. 2003, Namgail et al. 2004, Pozo \& Wilmer 2004). If species are not sufficiently differentiated in their ecological demands to reduce interspecific competition, coexistence requires explanation. Lotka (1925) and Volterra (1926) proposed a general framework that explains interspecific coexistence of competitors: when the level of interspecific exceeds that of intraspecific competition, the superior species would displace the inferior one by suppressing resources (Hardin 1960, Holt et al. 1994). Numerous empirical studies supported the "Lotka-Volterra" model: some fish species, for instance, coexist only if falling below a critical level of ecological similarity, whereas in case of extensive niche overlap, interspecific competition increases mortality rate in the inferior species (Jensen 1997). Continuous elaboration of the model since its proposal afforded opportunity to further examine mechanisms of interspecific coexistence. Recent work by Murrell \& Law (2003) suggested, for instance, that coexistence rather depends on the relative interaction distance of interspecific vs. intraspecific competition than on their relative strength.

The "Lotka-Volterra-Model" gave rise to the principle of competitive exclusion and the so-called "assembly rules" that were proposed to predict the spatial distribution of coexisting species (e.g. Diamond 1975, May 1981). "Forbidden species pairs", such as competitors, were empirically found in negative association, e.g. in island-living birds (Diamond 1975; statistical re-analyses: Sanderson et al. 2009, but see also Connor \& Simberloff 1979, 1983). A global empirical study matched the predictions of Diamond's model in that fewer species combinations, more species pairs in mutual spatial exclusion ("checkerboard" distribution), and less co-occurrence is observed in natural systems than expected by a null model without any interspecific interactions (Gotelli \& McCabe 2002).

Due to phylogenetic conservation of traits, closely related species are often ecologically similar, and competitive interactions are therefore of particular significance in the organization of taxonomic assemblages (sensu Pianka 1973). Interspecific competition may prevent coexistence of closely related species, and coexisting species are therefore more distantly related than expected by chance (phylogenetic evenness: Sligsby \& Verboom 2006, Lessard et al. 2009). The ecological structure of taxonomic assemblages was intensely investigated and competitive exclusion found in many cases (e.g. seed-eating desert rodents: Brown 1973, Brown \& Liebermann 1973, Bowers \& Brown 1982; snails: Brown 1982; tadpoles: Wilbur 1982). Accordingly, assemblages of more than two congeneric species are 
generally rare (e.g. birds: Kullberg \& Ekman 2000). In primates, congeners coexist relatively rarely, and more closely related taxa, such as sister species, are hardly ever found (Houle 1997).

However, the level of competition among closely related species does not necessarily exceed that between phylogenetically distant organisms (Schoener \& Spiller 1987, Morin et al. 1988, Umapathy \& Kumar 2000, Hickerson et al. 2004). In many assemblages, interspecific competition among congeners was not more pronounced than among rather distantly related species, and sympatric species were even more closely related than expected from random assortment (paleartic and British mammals: Letcher et al. 1994, isopods: Sfenthourakis et al. 2005, dyscids: Vamosi and Vamosi 2007). In addition, mammals with less overlap in space than predicted by chance, were more similar in body size and vice versa. Thus, sympatry among closely related species seemed facilitated by overlapping ecological requirements, whereas distributional overlap in distantly related organisms was reduced by competition (Letcher et al. 1994). This pattern arises due to structuring processes operating simultaneously at different levels: organisms assembling within a community were found to be structured by "trait-based assembly rules", whereas the actual representatives of a trait-based species pool were determined rather by priority effects from early colonization (Fukami et al. 2005). Kraft et al. (2008) proposed two simultaneous nichebased processes structuring communities: convergence of coexisting species due to environmental filtering of organisms adapted to the respective habitat ("unifying environmental filtering") and divergence due to competition leading to ecological differentiation ("competition-based differentiation").

\section{Direct interspecific effects: Predation}

Predation often has a greater impact than resource competition on the size and stability of prey populations, and consequently on community structure (Paine 1966, Sih et al. 1985 in Hart 2007). The intensity of predation influences the degree of overlap and intensity of competition between prey species (Glasser 1979, Holt 1984). Competition and predation should therefore be regarded as complementary mechanisms structuring ecological communities, which can lead to spatial patterns among prey similar to those resulting from competition. Predation does not necessarily make coexistence of prey species more likely, however. In many natural communities, predators were found to stably exclude species, 
whereas in others, predators were crucial in facilitating coexistence of competitively incompatible species (Holt 1984).

\section{Indirect interspecific effects}

Aside from direct interspecific effects, numerous indirect interactions shape ecological structure. Indirect effects are defined as "how and to what degree pairwise species interactions are influenced by the presence and density of other species in the community" (Abrams 1987; in Strauss 1991). The significance of indirect interspecific regimes for ecological structure becomes apparent where species have disappeared that originally structured a community. In consequence of local extinction of North American wolves (Canis lupus), for example, the population of coyotes (Canis latrans) increased to densities now threatening San Joaquin kit foxes (Vulpes macrotis mutica; Linnell \& Strand 2000). Moreover, removal of keystone predators may not only alter community structure within a trophic level (such as among prey), but impacts the entire underlying food web across trophic levels (Estes \& Palmisano 1974; Strauss 1991).

Some authors even consider indirect effects to be the unit interaction of community evolution (Strauss 1991). Interspecific competition, for example, was traditionally considered a direct interaction, but was then interpreted to operate indirectly as it is most frequently mediated by indirect exploitation rather than by direct interference (Schoener 1983, in Strauss 1991). Aside from exploitative competition, apparent competition and intraguild predation are among the most prominent phenomena of indirect interactions (Strauss 1991). Apparent competition generates spatial patterns similar to those resulting from competition (Holt \& Lawton 1994, Bonsall \& Hassell 1997, Bonsall \& Holt 2003). If two prey species do not compete for resources but share a predator, which numerically responds to the size of prey populations, this may result in reduced population growth and eventual disappearance of the more vulnerable prey (Holt 1984, Bonsall \& Holt 2003). Intraguild predation occurs if predator and prey belong to the same trophic guild, so that they affect each other by competition, in addition to the direct effects of predation. Coexistence is stabilized if the prey species in intraguild predation represents the superior competitor in resource exploitation, whereas the predator considerably gains from the consumption of the prey (Polis et al. 1989, Polis \& Holt 1992, Holt \& Polis 1997). 
However, frequency- or density-dependent predation can also indirectly stabilize coexistence among competitors (Amarasekare 2003). Theoretical (Caswell 1978; Glasser 1983) as well as empirical studies (Estes \& Palmisano 1974) have shown that predators can increase diversity of prey species by preventing competitive exclusion among them. As predators usually favor one particular species among a set of potential prey, either via specific preference or density dependence (Holt \& Lawton 1994, Chesson 2000a), they reduce the relative abundance of the preferred prey (Sundell et al. 2003). If the competitively superior prey is affected, predators can indirectly prevent competitive exclusion of an inferior competitor (Paine 1966, Caswell 1978, Holt et al. 1994, Kullberg \& Ekman 2000). Even if predation risk is shared, negative effects of predation on a species can be coupled with an indirect positive effect of a competitor being consumed (Wilbur \& Fauth 1990; Strauss 1991). Moreover, predation can lead to stabilizing coexistence of prey species that outcompete each other in different tasks, such as resource competition and predator avoidance (e.g. Chesson 2000a, b). The species more susceptible to predation occurs in highest densities in predator-free space, whereas the superior competitor's population peaks in most productive habitats. Local aggregation of individuals causes negative intraspecific interactions to increase relative to interspecific interactions and competitive exclusion is prevented (Amarasekare 2003). Stabilizing coexistence may also occur between intraguild-predator and prey, if the prey species is superior in competition for a shared resource, whereas the predator species significantly gains from its consumption of the prey species (Palomares \& Caro 1999, in Linell \& Stand 2000).

\section{Consequences of habitat characteristics for ecological structure}

Interspecific interactions are highly dependent on habitat suitability, which is rarely homogeneous across the total range of a species (Ylönen et al. 2003). As populations track spatial variations across a species' biogeographic range, populations tend to be most abundant in most suitable core areas far from the range boundary (Brown et al. 1995, Swihart et al. 2003, Swihart et al. 2006). If species respond differentially to distinct habitat types, the outcome of interspecific competition may vary considerably. Productive habitats allow for coexistence of ecologically similar species, whereas less productive habitats promote competitive exclusion (Brown \& Liebermann 1973). Superiority of a species in certain habitat types will lead to local displacement of the inferior species (Amarasekare \& 
Nisbet 2001, Amarasekare 2003, Aunapuu \& Oksanen 2003), but coexistence can still be favored on a regional scale by spatial heterogeneity. Refuges allow the inferior species to escape competition (Bonsall \& Holt 2003, Amarasekare 2003, Schreiber \& Kelton 2005, López-Gomez \& Molina-Meyer 2006) or predation (Paine 1974, Wellborn 2002, Ylönen et al. 2003). If competitive rankings are reversed in heterogeneous habitat, each species will be interspecifically excluded from an unfavorable habitat type, and be forced to the more favorable one (spatial storage effect: Chesson 2000b).

Habitat heterogeneity also involves temporal autocorrelation in environmental variables such as climatic conditions (Petchey et al. 1997). Environmental harshness and fluctuating conditions have been proposed to reduce the importance of interspecific interactions, such as competition, and consequently to promote coexistence of even ecologically very similar species. However, closer examination revealed that coexistence is not necessarily facilitated by harsh and fluctuating conditions, but only if spatial or temporal niche space is created by environmental conditions (Chesson \& Huntly 1997).

\section{System stability in view of habitat change}

In addition to intrinsic characteristics, the stability of ecological systems considerably depends on environmental conditions (Reichholf 1993), which is of great concern in view of globally proceeding anthropogenic disturbances. Habitat loss and fragmentation are the most important factors causing extinctions (Fischer \& Lindenmayer 2007, Irwin et al. 2010), and are therefore considered as major threats to biodiversity in general (e.g. Swihart et al. 2001) and to primate persistence in particular (Irwin \& Raharison 2009).

Habitat destruction can influence populations on various levels, such as on individual behavior or the populations' local dynamics and genetic structure. Populations do not necessarily respond linearly to habitat loss and fragmentation, but can decline abruptly over a narrow range of habitat impairment when extinction thresholds are exceeded (With \& King 1999). Likewise, species that are subject to the same disturbances do not always react in a similar manner, even if phylogenetically closely related (Irwin et al. 2010). Asymmetric effects on coexisting competitors or on a predator and its prey may alter the strength or even type of interspecific interactions and potentially result in "higher-order effects". Higher-order effects are distinguished from indirect interactions in that they change 
population dynamics and challenge the community structure more profoundly and in more unpredictable ways (Billick \& Case 1994, Swihart et al. 2001).

Established systems of coexisting species that are subject to profound environmental change may either collapse or reach an alternate state (Tokeshi 1999). Ecological communities were traditional believed to implement one of multiple stable climax states (Clements 1936 in Stokstad 2009, Lewontin 1969 and May 1977, both in Petraitis et al. 2009). According to this notion, communities subjected to environmental change would shift from one alternate stable state to another. Recent experimental work gave evidence for multiple alternate states and rapid shifts from one state to another in intertidal communities, with changes often being irreversible even if original conditions were restored (Petraitis et al. 2009). However, prevailing theory assumes communities rather to represent open dynamic systems than to shift between alternate stable states (e.g. Parrot 2002, Beisner et al. 2003). In such dynamic systems it may take considerable time to reach a new equilibrium after disturbance (Hanski 1997). Due to the variety of factors determining community composition, it cannot be easily predicted whether a successive community will fall into a new state (Stokstad 2009).

\section{Anthropogenic impact on habitat structure and suitability}

In vertebrates, patch occupancy rates increase with primary forest cover, which is characterized by high carrying capacities. Moreover, primary forests have a greater potential than secondary forests to support specialist species (Swihart et al. 2006). Loss of habitat suitability directly threatens the stability of established systems if the ecological needs of community members are no longer satisfied. Community deprivation is considered to be caused by loss of structural complexity and floristic diversity in anthropogenic environments (Pardini et al. 2005, Bisseleua et al. 2009). These habitat features decline along a coarse gradient from near-primary to secondary forest, agroforestry systems, plantations, arable crops, and pastures (Schulze et al. 2004, Basset et al. 2008, Scales \& Marsden 2008) and provide a crude proxy of biodiversity value across land-use intensification gradients (Gardner et al. 2009). Secondary habitats resulting from anthropogenic disturbances may look similar to undisturbed habitat, but essentially differ in structure and plant composition (Brown \& Gurevitch 2004, Irwin et al. 2010). Therefore, many forest-adapted species are excluded from communities that succeed disturbances (Bihn et al. 2008). 
Habitat degradation is often associated with spatial subdivision. Resulting patches differ in content, i.e. in vegetation structure and species composition, as well as in context, i.e. in size and position relative to other patches or to essential habitat features. Interactions of content and context determine the distribution of small mammals in fragmented landscapes (Schweiger et al. 1999, Fischer \& Lindenmayer 2007). In small and isolated fragments, abundance and alpha-diversity are lowered (e.g. Pardini et al. 2005) due to several detrimental effects. First, altered patch content, such as loss of particular species, can change the intensity of interspecific interactions and disrupt ecological structures. Second, the patch context affects movements on different scales, ranging from daily movements, over juvenile dispersal or seasonal migration to large-scale movements such as range shifts in response to climate change (Soulé et al. 2004). In addition to determinative effects, populations in isolated patches are, third, prone to extinction in consequence of environmental, demographic, as well as genetic stochasticity, which is irreversible if adjacent populations are too remote or specific dispersal distances too short to warrant recolonization (Lande 1988, Howe \& Davis 1991, Ims \& Yoccoz 1997, Stacey et al. 1997).

\section{Specific sensitivity to habitat loss and degradation}

Anthropogenic impacts are numerous and affect different species in non-uniform ways, even if phylogenetically closely related (Fischer \& Lindenmayer 2007, Irwin et al. 2010). For some species, retaining tree cover in agricultural landscapes is sufficient to ensure persistence (Harvey et al. 2006, but see Philpott et al. 2008), whereas for others it is required to retain structural and floristic diversity in anthropogenically changed landscapes (Pardini et al. 2005, Bisseleua et al. 2009). Occurrence of North American gray squirrels (Sciurus carolinensis) in an agriculturally fragmented landscape was positively related to the size of remaining fragments, whereas sympatric red squirrels (Tamasciurus hudsonicus) could only persist on patches providing particular resources and were excluded from patches occupied by gray squirrels due to increased competition (Swihart et al. 2007). Isolation of patches additionally affected colonization abilities in some squirrel species (S. carolinensis, Glaucomys volans), in which community structure was rather determined by metapopulation dynamics than by interspecific competition (Swihart \& Nupp 1998).

It becomes apparent that resistance to habitat loss and fragmentation, and therefore the probability of species persistence, differs considerably among coexisting species (Swihart 
et al. 2006). Several characteristics have been identified to make species vulnerable to anthropogenic disturbances (Fischer \& Lindenmayer 2007). Species with a wide biogeographical distribution, providing space for stable core populations, experience low levels of demographic stochasticity (Enquist et al. 1995), and are thus more resistant against anthropogenic disturbances (Swihart et al. 2003). In contrast, species with a narrow biogeographical range that consist predominately of unstable populations are prone to local and, if naturally rare, even global extinction events (Lawes et al. 2000, Nupp \& Swihart 2001).

Even more important for resistance against habitat disturbance in mammals, reptiles and amphibians are broad ecological niches (Swihart et al. 2006). Species capable of exploiting modified habitats were found to remain stable or increase in fragments, whereas those that avoid these habitats often disappeared (Laurance 1991). Accordingly, dietary generalists are less susceptible to habitat degradation than specialists are, as they can cope with an increased variation in food availability and extract some resources even from degraded habitat. Even if a greater flexibility in resource use may be an inferior strategy at a local level, it is advantageous on a regional level as it permits to respond to environmental changes and therefore favors persistence (Swihart et al. 2003). Moreover, generalists across taxonomic orders are capable of using human-altered landscapes for migration, and are therefore less vulnerable to habitat fragmentation. In contrast, specialists are particularly sensitive to fragmentation as they are largely restricted to remnant forest patches, and matrix habitat surrounding them often constitutes a migratory barrier (Sarre et al. 1995, Swihart \& Nupp 1998, Gascon et al. 1999, Bentley et al. 2000, Swihart et al. 2006). As specialists often have evolved in stable, homogeneous landscapes, they might not have developed dispersal strategies during evolutionary history. Strict arboreality, for example, decreases the chance of colonizing new patches in landscapes consisting of forest fragments separated by non-arboreous matrix habitat (Wolff 1999).

Additional behavioral characteristics, such as social organization, may determine a species' resistance to habitat fragmentation: species that use large home ranges or rely on patchy and unpredictable resources theoretically experience a greater risk of extinction in fragmented landscapes (Lawes et al. 2000). South African Samango monkeys (Cercopithecus mitis labiatus), for instance, are unable to colonize small isolated patches. This was explained by their gregarious social organization requiring large habitat patches as well as group 
dynamics that limit dispersal (Lawes et al. 2000). Therefore, individual requirement of territory or home range area represents a dominant characteristic for extinction risk, and dispersal ability accounts for recolonization of habitat patches (Vos et al. 2001).

Specific colonization abilities can also be limited by perceptual ranges, i.e. in the maximum distance from which individuals can perceive remote landscape elements such as most suitable habitat patches in terms of food supply, competition, or predation pressure. Perceptional ranges influence the probability of an individual to successfully disperse to a new patch, and therefore account for species-specific sensitivity to habitat fragmentation (Zollner 2000). The degree to which predators can track the distribution of prey, i.e. their functional or numerical response to prey occurrence, is dependent on numerous factors: aside from ecological constraints (niche breadth) and population growth, sensory capability and mobility determine the distribution of a predator. Behavioral or ecological characteristics of the prey, as well as characteristics of the environment can alter the prey's detectability and determine the tracking ability of predators (Swihart et al. 2001). Predators on a higher trophic level are particularly susceptible to habitat loss, if it changes the distribution and abundance of prey populations in a hardly predictable way (Wolff 1999, Swihart et al. 2003, Estes et al. 2011).

Habitat selection acts on various levels as a major mechanism structuring ecological communities (Lindenmayer et al. 2000). Thus, it is prudent to examine the role of structural habitat variables for community structure before invoking local interspecific regimes (Nupp \& Swihart 2001) that potetially interact with regional habitat selection (Gabor \& Hellgren 2000, Fukami et al. 2005, Kraft et al. 2008).

\section{Indirect effects resulting from habitat loss and fragmentation}

Given the complexity of ecological communities, any species' removal or addition will have indirect effects on multiple levels (Strauss 1991). If key species disappear as a result of habitat loss or degradation, the complex network of interspecific interactions might get out of equilibrium. This instability may threaten the system's identity and integrity (Gregorius 1996) and potentially results in extinctions of additional community members. Therefore, potentially interacting species are to be considered potential factors structuring communities if concerned with the fate of an endangered species (Diamond 1980a, b). Numerous studies have shown that reduction of habitat alters the level of interspecific 
competition and predation pressure (z. B. Hanski \& Ranta 1983, Kozakiewicz 1993, Creel \& Creel 1996, Creel 2001, Antón et al. 2005). Within isolated subpopulations, competitive pressure can increase to a level that causes local interspecific exclusion (Danielson 1992). Again, specialist species are more severely affected than generalists are, as reduced habitat area leaves a specialist with fewer options to maintain competitive superiority or escape predation (Swihart et al. 2003). In a metapopulation model, generalist predators were found less sensitive to habitat destruction than their prey, whereas specialist predators were driven to extinction by lower disturbance levels than their prey. Consequently, generalist predators facing habitat destruction are likely proliferating at the expense of specialists.

Extinction of predators intensifies the impact of habitat loss on regional abundance of prey species, which are consequently threatened with local extinction (Estes et al. 2011). Particularly species that serve only as incidental prey for generalist predators (constituting only minor components of the diet), were found more imperiled than predators by habitat degradation and face the greatest risk of extinction (Swihart et al. 2001). Habitat fragmentation can additionally aggravate the effects that predators exert on prey populations. Whereas vacant ranges in continuous forest would get repopulated over time, recolonization of remote fragments is less likely and predation therefore accelerates extirpation from patches that could otherwise sustain populations (Irwin et al. 2009)

Despite numerous risks, habitat fragmentation also has the potential to promote persistence of subdivided populations (Petchey et al. 1997). By increasing environmental heterogeneity, and thereby altering the stability of species interactions, spatial subdivision was found to create opportunities for coexistence in both, competitive as well as predatorprey systems (Kareiva 1990). White-footed mice (Peromyscus leucopus), for instance, which occupied smaller patches within a fragmented landscape were released from interspecific competition with larger granivores, whereas they have been excluded from larger patches (Nupp \& Swihart 2001).

Predicting what equilibrium state a species assemblage will reach after environmental changes is of crucial interest to conservation biologists. Unfortunately, no consensus was achieved on the relative importance of determining factors despite intense theoretical and empirical investigation of ecological structure. Due to the manifold, potentially co-varying, factors structuring communities, species compositions are highly diverse, and it is therefore hardly feasible to extrapolate from one system to another. In a 
study of plants in similar salt marsh communities, for example, species assemblages were determined by physical stress in California, but instead by competition in Chile (Fariña et al. 2009). It is not even possible to predict if an established system of coexisting species suffering from habitat degradation will regenerate or whether intervention is required to prevent its falling to an undesirable new equilibrium (Stokstad 2009). However, even though general community rules do not provide fine-scale predictions on how species will assemble, conservation biologists still accept some broad principles as "hugely useful and critical for conservation" (Pimm cited in Stokstad 2009, Chase \& Leibold 2003).

\section{Model system: Sympatric cheirogaleids in Menabe Central, Western Madagascar}

\section{Forests of Madagascar}

As biodiversity in the tropics is most severely threatened, some authors propose to concentrate conservation efforts there (Tokeshi 1999). Madagascar obtained global conservation priority due to the island's exceptionally high rates of endemism and considerable anthropogenic threat (Mittermeier et al. 1992, Myers et al. 2000). Lowry et al. (1997) considers all vegetation in Madagascar affected to some extent by anthropogenic disturbances. The western dry deciduous forest, one of the most threatened ecosystems of the world and a Global 200 ecoregion (Dinerstein et al. 1996), has been most severely affected by human activities (Ganzhorn et al. 2001).

Habitat degradation by logging or subsistence agriculture generally reduces species diversity across all taxa, with most pronounced impacts on native and endemic species and characteristic species turnovers from specialists to generalists and from endemics to nonendemics (Irwin et al. 2010). One of the major processes impairing Madagascar's natural forests is habitat fragmentation (Green \& Sussman 1990), and fragment size was identified as primary factor determining the extent of species losses in Madagascar (Irwin \& Raharison 2009).

\section{Lemuriformes (Primates: Strepsirrhini)}

Lemurs, the strepsirhine primates endemic to Madagascar, qualify as model species for the examination of ecological structure in taxonomic assemblages. Lemur communities are characterized by high $\alpha$-diversity and often comprise closely related species. Compared with other primates, lemurs occur in high population densities, and therefore afford opportunity 
to study population and community ecology on a larger spatial scale (e.g. Mittermeier et al. 2008).

Despite high conservation priority and great research attention directed to lemurs, there is a lack of studies assessing lemur ecology and population health across disturbance gradients (Irwin et al. 2010). Studies that investigated lemur responses to anthropogenic habitat alteration found various reactions of lemur species to habitat fragmentation, ranging from positive to negative edge effects. Omnivores were often least affected by edges, whereas insectivores preferred this microhabitat due to high insect abundance (Lehman et al. 2006a). Susceptibility of lemurs to extinction from fragments increased with body mass and degree of frugivory (Irwin \& Raharison 2009), whereas resilience was promoted primarily by behavioral plasticity (Irwin et al. 2010). For the long-term conservation of lemurs, fragment sizes critical for community stability have been identified (e.g. $>1000$ ha for large bodied species: Ganzhorn et al. 2003). Intermediate disturbance levels may temporarily favor certain species and boost biodiversity for two main reasons (Ganzhorn et al. 1997, Irwin et al. 2010, Miller et al. 2011). First, moderate disturbances on a limited scale potentially increase forest productivity. Second, slight disturbances can mitigate the problem of "resource dilution" that exists in highly diverse forests and increases the effort to find essential resources (Ganzhorn et al. 1997). Secondary forests and anthropogenically altered habitats around forests were found to serve as buffer zones as they provide food for smaller, omnivorous lemurs. More intense degradations depriving habitats of essential structures usually cause species extinctions by food shortage or intensified negative interspecific interactions (Ganzhorn et al. 1997). Plantations can extend lemur habitat only if they have a dense understory providing accessible food, as well as travelling and resting facilities (Ganzhorn 1987, Ganzhorn et al. 1999). 


\section{Cheirogaleid assemblage in Menabe Central, Western Madagascar}

A taxonomic assemblage of closely related lemur species that occur sympatrically in dry deciduous forest of Central Western Madagascar qualifies for several reasons as a model system to investigate ecological structure against the backdrop of anthropogenic disturbances.

\section{Menabe Central}

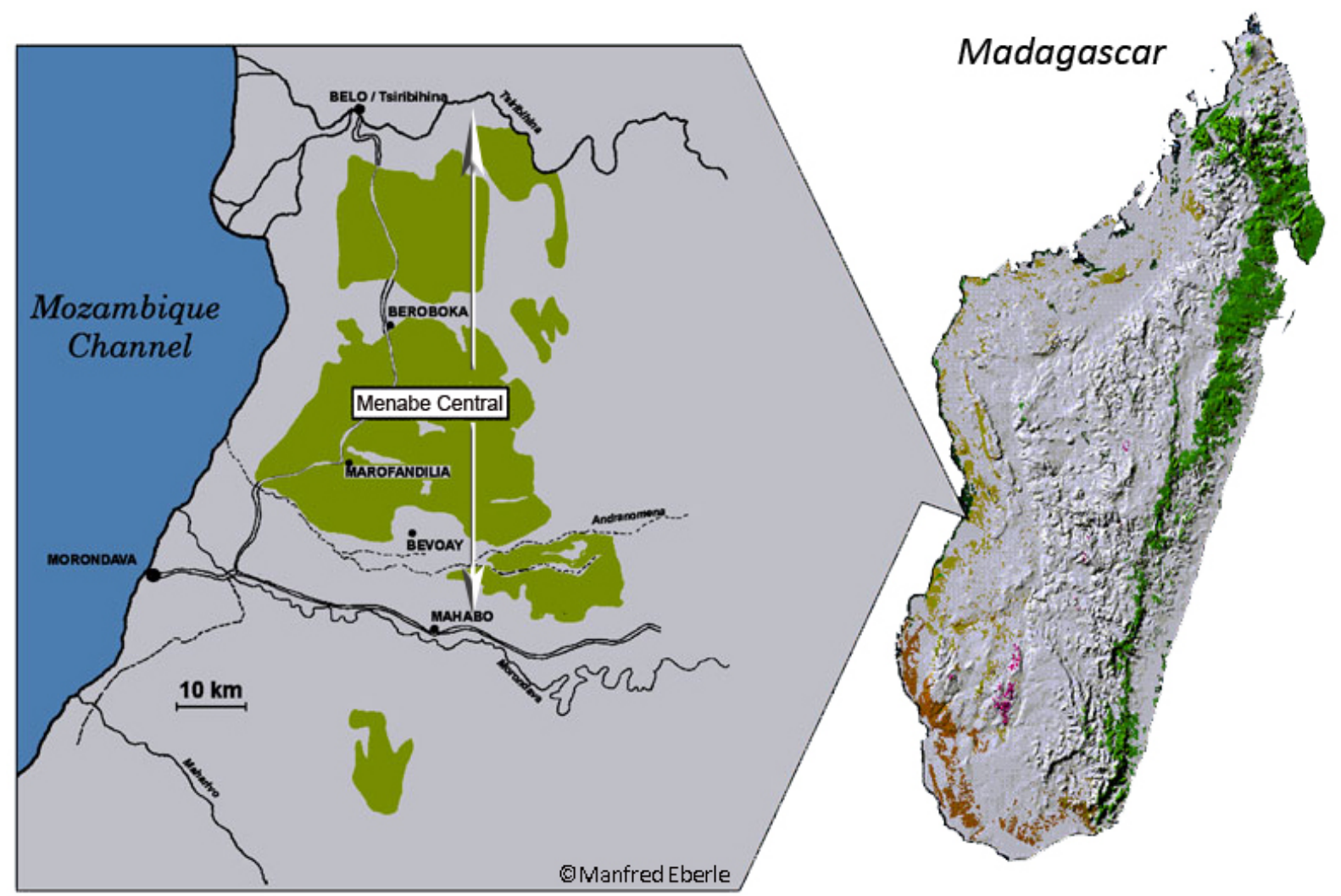

Fig. 1: Geographic position of the region Menabe Central

Menabe Central ranges from the Mozambique Channel to the bottom of the central highlands and is bound north-south wards by the rivers Tsirihibina and Morondava (figure 1). The region is of great significance to conservation for several reasons. With 125,000 hectares in total, Menabe Central retained approximately 65,000 hectares of forested area, which represents the largest remnant of dry deciduous forest in western Madagascar (Smith et al. 1997, Sorg et al. 2003). Menabe Central additionally outranks other forest areas by an exceptionally high rate of local endemism (including four locally endemic vertebrate taxa) and, therefore, has top conservation priority within one of the world's "hottest hotspots" (sensu Myers et al. 2000). 
Dry forest habitat in Menabe Central is subject to both, spatial and temporal heterogeneities. The climate is classified as tropical dry with a distinct dry season of 7-9 months (from March/ April to November)
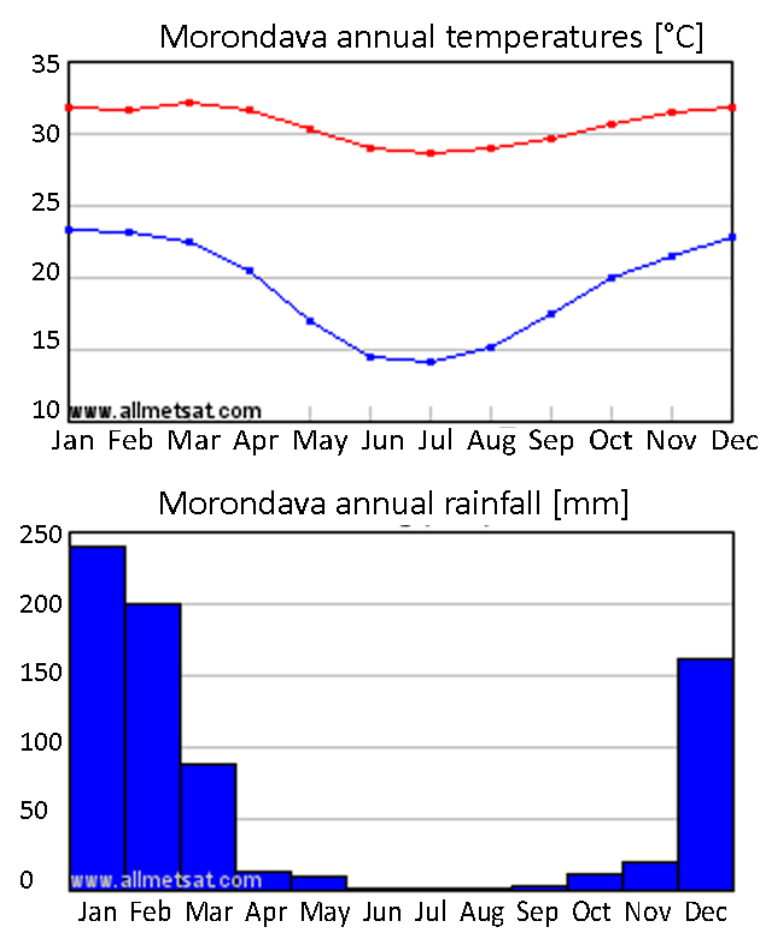
and a hot wet season of 3-5 months. The rainy season is characterized by sequences of wet periods, with heavy rains starting in December and gradually increasing to a maximum in January and February (Sorg \& Rohner 1996; figure 2).

Fig. 2: Climate diagrams for Morondava (from www.allmetsat.com)

The rural human population is comprised of several ethnic groups and concentrates in villages, which are spread out over Menabe Central. Humans frequent the forest in order to hunt for birds or mammals and to collect natural products for subsistence (Smith et al. 1997, Sorg et al. 2003, Réau 2002). Road building associated with oil exploration and logging has increased forest accessibility and facilitates exploitation (Smith et al. 1997). Most severe impacts result from slash-and-burn agriculture ("tavy"), which serves to create areas under crops or land used for zebu pasture. Tavy and illegal logging have reduced forest area at annual rates of $4-5 \%$, impairing habitat extent, connectivity, and quality. Excessive land clearances surrounding the village of Beroboka, originally intended for sisal plantation, have fragmented the forest. The northernmost forest part Ambadira has been segregated from Kirindy Forest over the past decades. At present, the two forest parts are only connected by a narrow corridor of 5-7km width. Anthropogenic disturbances in the environment of the village Marofandilia have been separating the Reserve Spécial d'Andranomena (RSA) in the south from the other forest parts. In areas with cleared forest, the vegetation consists of secondary forest formations, scrub, and savanna (Sorg et al. 2003). 

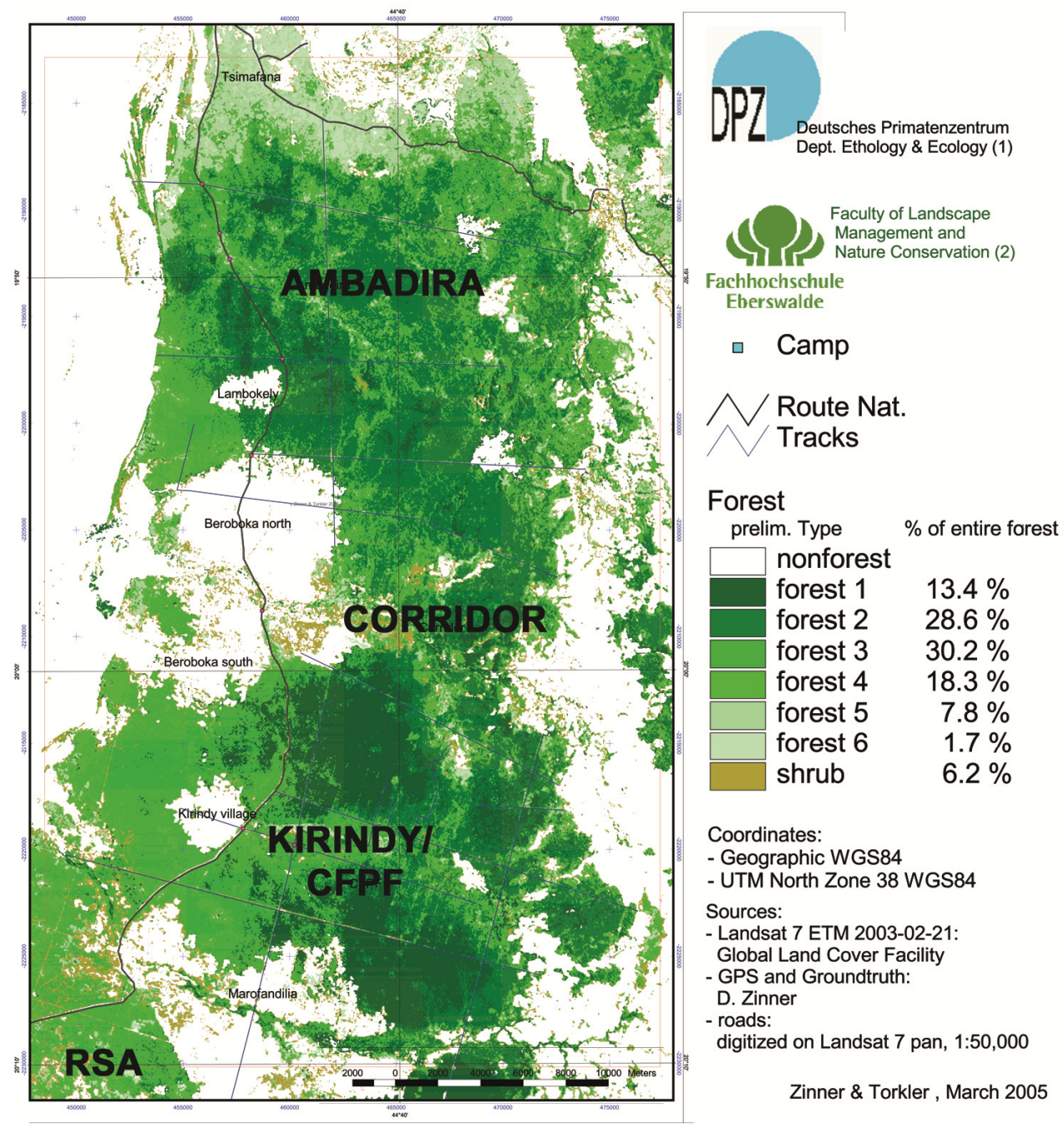

Fig. 3: Forest parts in Menabe Central (RSA only partially depicted)

Varying levels of anthropogenic pressure resulted in a mosaic of different vegetation forms between and within major fragments. Even though no strict primary forest remains in Menabe Central, some fragments still contain considerable areas of largely non-degraded habitat (figures 3 and 4). Ambadira Forest has never been effectively protected, but the region is only moderately accessed by major oil exploration tracks and contains considerable areas of near primary forest under moderate anthropogenic disturbances that promotes lemur species richness (Smith et al. 1997). The corridor, however, is highly frequented and has been continuously losing dry deciduous forest cover to scrublands (figure 74 in appendix, Zinner \& Torkler 2005). In Kirindy Forest, the Centre de Formation Proféssionelle Forestière (CFPF, nowadays CNFEREF) was granted a 12,500 ha forestry concession in 1978 for silvicultural research and selective logging that only slightly affected microhabitat structure (Ganzhorn et al. 1990). Since 1992, the CFPF's activities have been focused on sustainable forest management and ecotourism and a zoological research station was established by the German Primate Center (DPZ; Sorg et al. 2003). The presence of the CFPF and the DPZ has 
limited illegal activities within Kirindy Forest, but forest utilization is widely unrestricted in other parts of Menabe Central. Forestry concessions controlled by the national environment department "Eaux et Forêts", partly bordering the CFPF concession, have been illegally logged and there is evidence of hunting and timber harvesting along many of the roads traversing Menabe Central (Schäffler, pers. obs.). The Reserve Spécial d'Andranomena (RSA), is located in proximity to roads and villages and is therefore prone to clearing and other anthropogenic disturbances (Smith et al. 1997). Despite direct governmental control by the National Association for the Management of Protected Areas (ANGAP), more than two-thirds of the original forest cover has been heavily degraded or lost after the park's creation in 1958 (Randrianandianina et al. 2003).

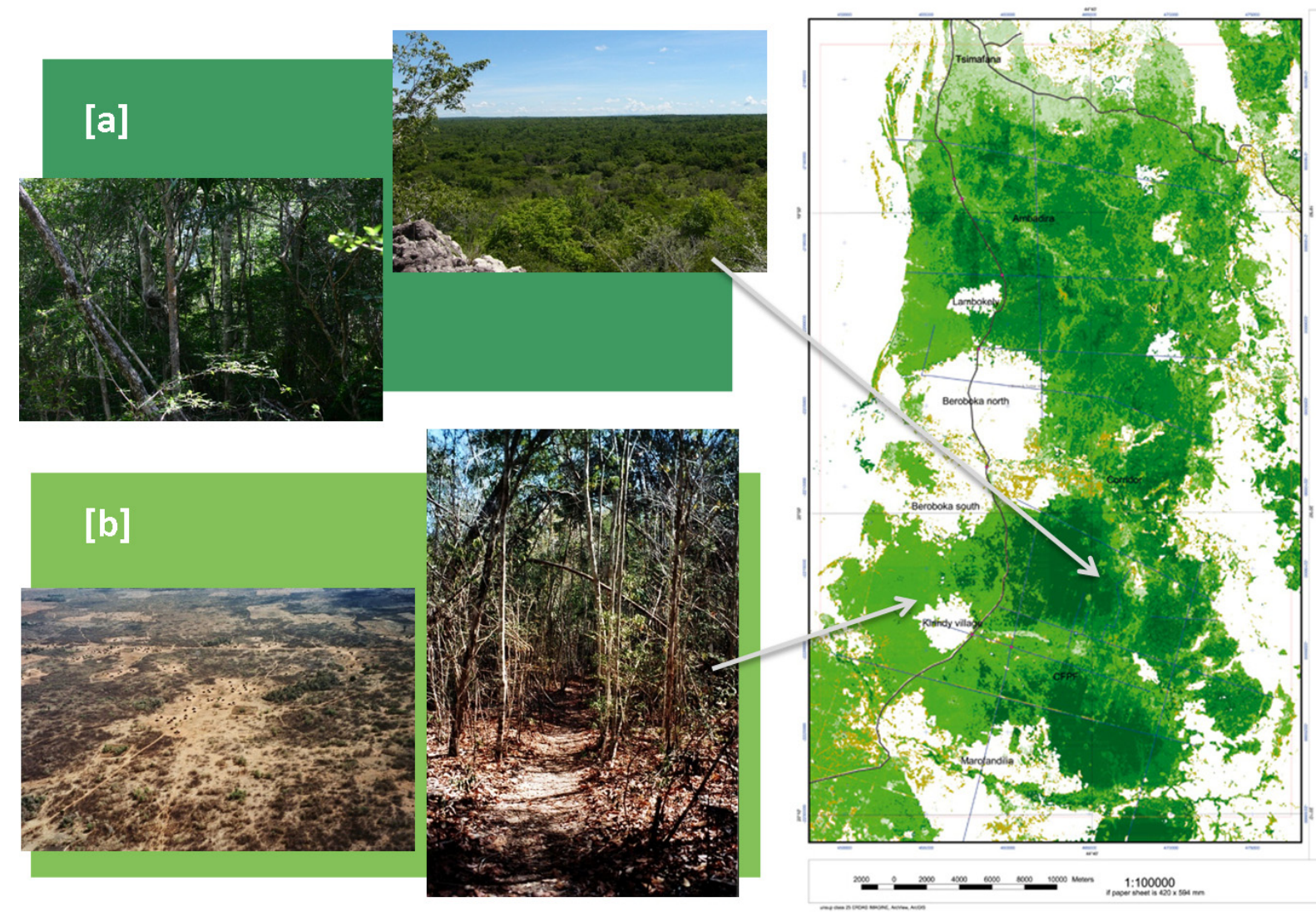

Fig. 4: Forest types resulting from anthropogenic impact in Menabe Central: [a] non-degraded and [b] degraded habitat (pictures: Henning Lahmann, Moritz Rahlfs, Livia Schäffler/ map: Zinner \& Torkler 2005)

\section{Sympatric cheirogaleids}

A taxonomic assemblage of five cheirogaleid lemurs (Lemuriformes: Cheirogaleidae) coexist in the dry deciduous forest of Menabe Central. Among those species is the smallest known primate of the world, Mme. Berthe's mouse lemur (Microcebus berthae (33g); figure 5), which was discovered only in 1992 (Schmid \& Kappeler 1994, Rasoloarison et al. 2000). 


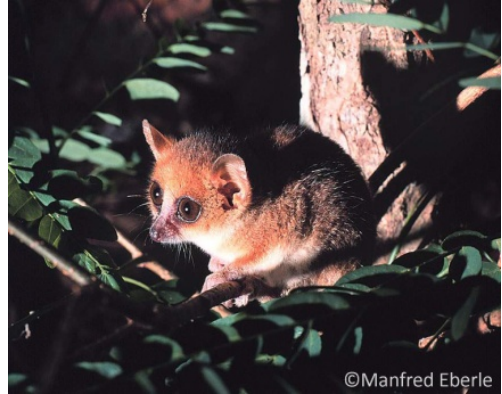

Fig. 5: Microcebus berthae

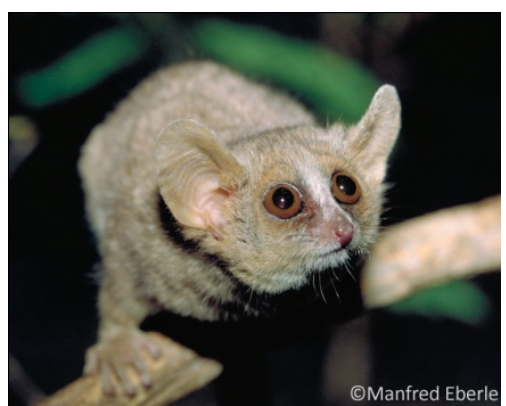

Fig. 6: Microcebus murinus

With its global range confined to Menabe Central (figure 7), M. berthae is considered biogeographically most restricted among all mouse lemurs (Rasoloarison et al. 2000, Schwab \& Ganzhorn 2004). The species' distribution corresponds to the confinement to areas of microendemism that is characteristic for most mouse lemurs (Wilmé et al. 2006).

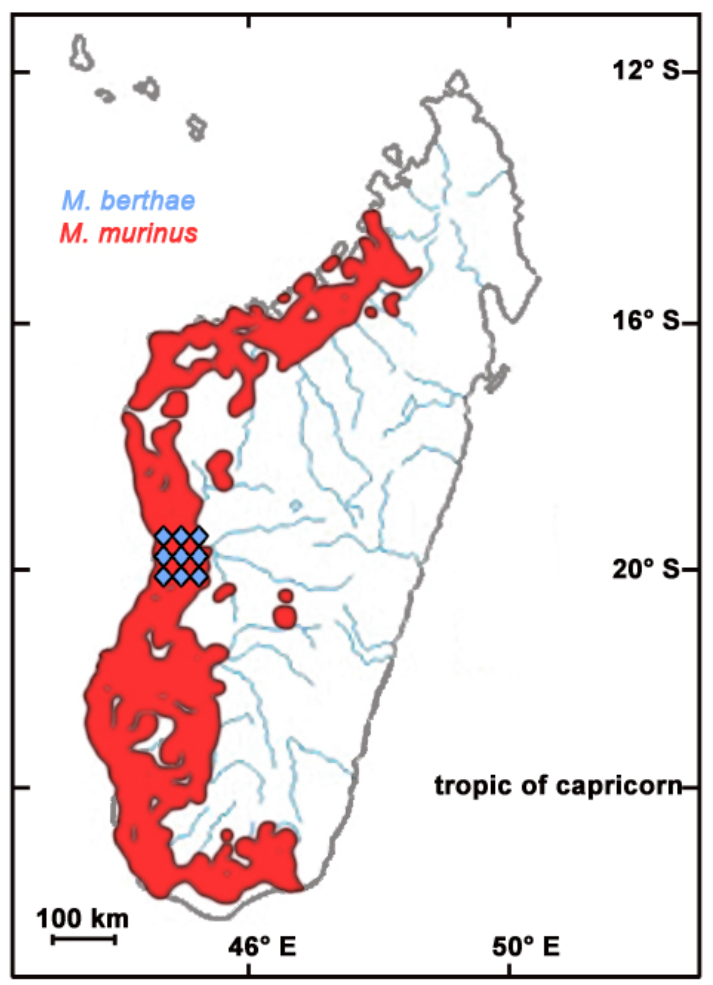

Fig. 7: Global distribution of $M$. berthae (blue) and M. murinus (red)

In Menabe Central, M. berthae coexists with the gray mouse lemur (M. murinus (60g): figure 6; Schmid \& Kappeler 1994, Rasoloarison 2000, Kappeler \& Rasoloarison 2003, Schwab \& Ganzhorn 2004). Microcebus murinus occurs across the entire western and southern part of the island (figure 7) and its biogeographic distribution overlaps with the more restricted ranges of several other mouse lemurs (Schmid \& Kappeler 1994, Weisrock et al. 2010). The congeners belong to different phylogenetic subclades (Yoder et al. 2000), indicating allopatric speciation and secondary coexistence after long periods of independent history. 
Microcebus spp. do not comply with ecological similarity sensu Brown (1984) considering the differences between the mouse lemurs' biogeographic ranges as well as population densities. Spatially limited studies in Kirindy Forest indicated that Microcebus spp. both are inhomogeneously distributed, but differ in population densities (e.g. Ganzhorn 1991, 1994, Eberle \& Kappeler 2002, 2004a, b, 2006, Schwab \& Ganzhorn 2004, Fredsted et al. 2004, 2005, Dammhahn \& Kappeler 2005, 2008, 2009, 2010).

Ecological differences between $M$. berthae and $M$. murinus are also reflected in diverging susceptibilities to anthropogenic disturbances. As a locally endemic species with a small biogeographic range that is subject to anthropogenic disturbances, $M$. berthae meets the conditions for critical conservation (Myers et al. 2000) and was rated endangered in the IUCN list of threatened species in 2008 (Andrainarivo et al. 2008, in IUCN 2010). As proximity to the range boundary and niche breadth substantially determine vertebrates' sensibility to habitat alteration (Swihart et al. 2003), M. berthae is likely more affected by anthropogenic disturbances than generalist $M$. murinus. In Menabe Central, M. berthae was found only on habitat patches larger than 30.000 hectares (Ganzhorn et al. 2003), indicating high susceptibility to forest fragmentation. In contrast, gray mouse lemurs are rated as of "least concern" in the IUCN list of (Andrainarivo et al. 2008, in IUCN 2010) owing to their wide distribution, high abundance and resistance to habitat alteration (Ganzhorn et al. 2003). Microcebus murinus has been observed in all forest types and any fragment exceeding one hectare, including agricultural and village environments (Ganzhorn et al. 2003). In primary forest, M. murinus prefers areas with low tree diversity, presumably to avoid the problem of resource dilution, whereas in secondary forests it occupies ranges characterized by a minimum number of essential tree species (Ganzhorn et al. 1997, cf. Lehman et al. 2006a). However, other characteristics of degraded forest appear to limit habitat value even for disturbance-tolerant $M$. murinus: females outlast the dry season by entering energy-saving torpor (Martin 1972, Petter et al. 1977, Petter-Rousseaux 1980, Schmid 1996, 1999, 2000a, b, 2001, Ganzhorn \& Schmid 1998, Schmid et al. 2000, Schmid \& Speakman 2000) and their capacity to enter daily torpor was found constrained by fewer resting holes and higher ambient temperatures in secondary habitat. A lower body mass and increased mortality risk in secondary compared to primary forest underscores the limited value of degraded habitat to M. murinus (Ganzhorn \& Schmid 1998). 


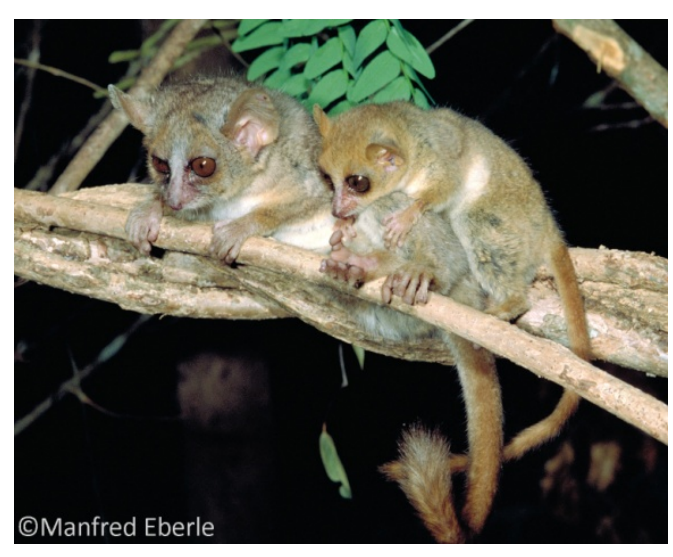

Fig. 8: Body sizes of M. murinus (left) and M. berthae (right)

Interspecific body size ratio of Microcebus spp. slightly exceeds the Hutchinsonian ratio for "limiting similarity" and therefore indicates ecological differentiation (Hutchinson 1959, MacArthur \& Levins 1967; Figure 8). Even though both Microcebus spp. are omnivorous and experience similar seasonal fluctuations in food supply (Schwab \& Ganzhorn 2004), differential feeding niches are reflected in the intraspecific distribution of individuals: Microcebus berthae uses larger home ranges with a mean area of 2.5 hectares almost exclusively, which is associated with scramble competition for dispersed resources. In contrast, M. murinus competes for clumped resources and occupies smaller individual home ranges (1.3 ha) that considerably overlap between individuals (Eberle \& Kappeler 2002, Dammhahn \& Kappeler 2009, 2010).

However, comprehensive examinations of Microcebus spp.'s resource and habitat utilization on a local scale concluded that interspecific coexistence could not be explained merely by ecological differentiation (Dammhahn \& Kappeler 2008). Feeding niche overlap was shown to be high in terms of food categories (fruits, flowers, tree exudates, insects and insect secretions, small vertebrates), but the mouse lemurs differ in the proportions of food categories used. Microcebus berthae relies mainly on insect material and its narrow feeding niche is embraced by the wider niche of $M$. murinus. The gray mouse lemurs' diet includes higher amounts of fruit and gum in addition to animal matter, which are subject to greater seasonal variation (Dammhahn \& Kappeler 2010).

During the dry season, secretions by larvae of the homopteran insect Flatidia coccinea (Homoptera, Fulgoridae) comprises up to $82 \%$ of $M$. berthae's overall diet and is further supplemented by insect material (Dammhahn \& Kappeler 2008, 2010). Microcebus murinus females also rely on homopteran secretions during the scarce dry season when 
other sugary food sources such as fruits and nectar are absent or less abundant (Corbin \& Schmid 1995). Flatidia live in large colonial aggregates and feed on the liana Elachyptera minimiflora. Larvae are present throughout the dry season (April to November) and produce sugar-rich liquid secretions which drip onto leaves and stems, drying into a white solid that can be licked or scraped directly from the vegetation (Hladik et al. 1980). Homopteran secretions were found more abundant along edges than in the forest interior in Kirindy Forest (Corbin \& Schmid 1995). However, the microhabitat utilization of Microcebus individuals did not correspond to the distribution of liana, which are related this essential resource (Dammhahn \& Kappeler 2008).

Mouse lemurs are identical in three-dimensional use of space (Reimann 2002), but they might have divergent microhabitat requirements in consequence of differential resting behavior: Microcebus berthae builds nests from liana, whereas $M$. murinus sleeps in tree holes. However, closer examination of microhabitat utilization by the two mouse lemurs in Kirindy Forest did not indicate specific preferences for structural habitat characteristics related to either nesting material or sleeping sites (Dammhahn \& Kappeler 2008, cf. Rendigs et al. 2003, Reimann et al. 2003).

Microcebus spp. are also subject to a similar top-down control, all facing intense predation pressure from a great variety of predators that range from nocturnal and diurnal raptors, over snakes to carnivorous mammals (Goodman et al. 1993, Goodman 2003, Scheumann et al. 2007). Given this diversity of predators, a variety of behavioral strategies are presumably required to reduce the risk of being captured, e.g. in relation to social organization, daily and seasonal inactivity, concealment and vigilance, escape or mobbing behavior (Goodman et al. 1993a, Scheumann et al. 2007, Karpanthy \& Wright 2007). A predation rate of $25 \%$ in $M$. murinus, which was estimated for Beza Mahafaly and Berenty Reserve has been considered the highest known in primates (Goodman et al. 1993). Long-term capture-mark-recapture studies in Kirindy Forest even documented mortalities of up to $50 \%$ in $M$. murinus and up to 70\% in M. berthae (Dammhahn \& Kappeler 2008).

Predator-mediated coexistence of Microcebus spp. in Kirindy Forest was excluded due to assumed similarity in predation pressure and the same seasonal variations in predation risk (Schwab \& Ganzhorn 2004), but particularly as M. berthae's mortality appeared to exceed that of superior competitor M. murinus (Dammhahn \& Kappeler 2008). 
Given the variety of potential anti-predator strategies and the high selection pressure, mouse lemurs may likely have evolved divergent anti-predator behavior strategies and therefore be differentially affected by various predators. Moreover, not only predators have the potential to stabilize interspecific coexistence, but also third agents that are engaged in competitive or intraguild-predatory interactions. Interactions with third agents have never been examined as a mechanism stabilizing interspecific coexistence of Microcebus spp. In order to narrow this informational gap, we examined the spatial distribution of coexisting cheirogaleids in addition to habitat variables as potential determinants of population structure in mouse lemurs.

The taxonomic assemblage of cheirogaleids in Menabe Central comprises three family members in addition to mouse lemurs: the fat-tailed dwarf lemur (Cheirogaleus medius, Figure 9), Coquerel's dwarf lemur (Mirza coquereli, Figure 10), and the fork-marked lemur (Phaner pallescens, Figure 11). Considerably exceeding Microcebus spp. in body size, those species are referred to as "greater cheirogaleids" in the following. Cheirogaleus medius and M. coquereli might potentially influence the population ecology of Microcebus spp., as they considerably overlap with mouse lemurs in their feeding niches and in three dimensional use of space (Hladik et al. 1980, see also Lahann 2008). In contrast, an impact of $P$. pallescens seems rather unlikely due to pronounced ecological differentiation by various means: Phaner is highly specialized on gum exudates (Combretaceae: Terminalia) and its activity range is vertically separated from those of its family members (Hladik et al. 1980, CharlesDominique \& Petter 1980, Schülke 2003).

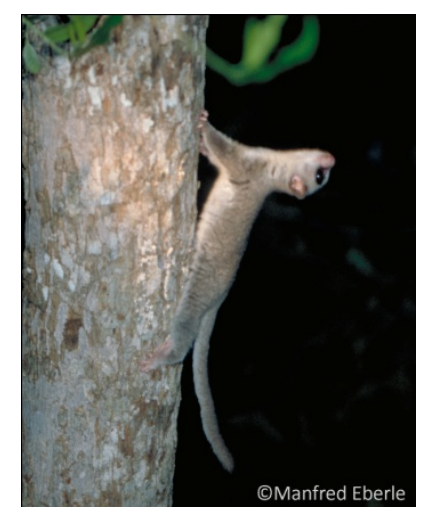

Fig. 9: Cheirogaleus medius

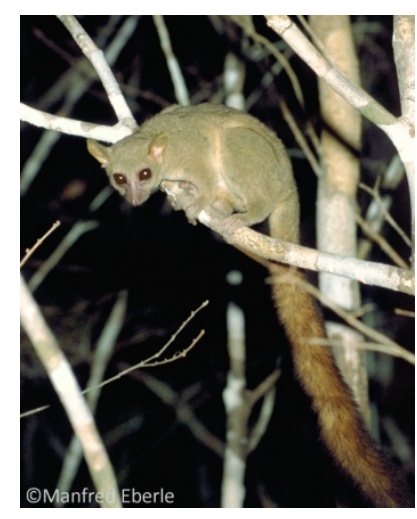

Fig. 10: Mirza coquereli

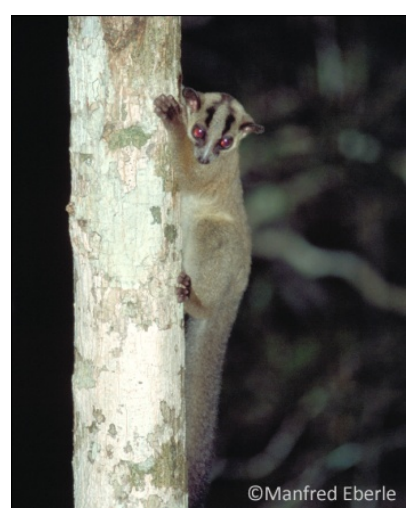

Fig. 11: Phaner pallescens 
Cheirogaleus medius considerably overlaps with Microcebus spp. in its feeding niche as well as in structural habitat requirements (Hladik et al. 1980). The genera were found in negative spatial association in an early survey within Kirindy Forest (Ganzhorn \& Kappeler 1996). Cheirogaleus medius outcompetes M. murinus in the feeding context (Ganzhorn \& Kappeler 1996) and both species use tree holes for resting. In result of competitive superiority $C$. medius has the potential to displace M. murinus (Ganzhorn \& Kappeler 1996) to thereby lower competitive pressure on M. berthae. Schwab \& Ganzhorn (2004) found C. medius to partially displace $M$. murinus on a local scale, whereas positive spatial associations with $M$. berthae indicated relaxed resource competition in consequence of differential habitat utilization patterns. Both sexes disperse in C. medius, even though covered distances are so short that even adjacent populations are genetically substructured (Fredsted et al. 2007). In case of habitat fragmentation, $C$. medius would therefore likely suffer from inbreeding due to limited gene flow between subpopulations and experience a high risk of local extinctions due to demographic chance effects (e.g. Lande 1988). This is in accordance with the fact that C. medius was not found in fragments of dry deciduous forest smaller than 600 hectares (Ganzhorn et al. 2003). However, the species has been recently assessed to be of "least concern" (Andrainarivo et al. 2008, in IUCN 2010), as it is believed to remain widespread and abundant, with none of the major threats resulting in significant range-wide population declines.

All three species interact with $M$. coquereli: primarily feeding on fruits, gum, flowers and young leaves as well as animal matter, the species' considerably overlaps with Microcebus spp. and C. medius in its dietary niche (Goodman et al. 1993, Goodman 2003). During the scarce dry season, $M$. coquereli relies on secretions of homopteran larvae and tree exudates (Pages 1980, Kappeler 2003) and competes with Microcebus spp. for this essential resource. Moreover, there is anecdotal evidence for predation on M. murinus (Goodman 2003, Kappeler 2003, Hart 2007, Schliehe-Diecks et al. 2010) and in confrontation experiments, $M$. coquereli evoked anti-predator responses in $M$. murinus individuals (Rakotonirainy 2003, Fichtel 2009). Predation by M. coquereli on M. berthae is not reported in the literature. Intra-guild predation by omnivorous $M$. coquereli qualifies as a mechanism to stabilize coexistence of Microcebus spp., irrespective of the question whether the prey species is selected by specific preference or in a density-dependent manner. In case of density-dependent predation, $M$. coquereli would capture disproportionally more $M$. 
murinus individuals, which occurs in higher population densities than $M$. berthae. Gray mouse lemurs may also be easier to detect as individuals are clumped in space. Mirza is omnivorous and not specialized on vertebrate prey, so predation pressure on M. murinus should only be significant when resources are scarce, i.e. in degraded habitat and during the dry season (cf. Schliehe-Diecks et al. 2010). In aggressive interactions, M. coquereli appeared competitively inferior to C. medius (Ganzhorn \& Kappeler 1996). Based on phylogenetic diversity, M. coquereli was considered of highest conservation priority (Lehman 2006a). Population reduction of $20-25 \%$ over the past fifteen years was attributed to substantial loss and degradation of habitat across the entire range of the species. In addition, fragmentation increasingly impairs $M$. coquereli and its conservation status was accordingly assessed as "near threatened" in the IUCN Red List (Andrainarivo et al. 2008, in IUCN 2010). On a regional scale, M. coquereli inhabits forest fragments down to a size of 6 hectares (Ganzhorn et al. 2003). Migration between local populations is essential to the species long-term persistence as it allows for recovering from local population declines (Kappeler et al. 2002, Markolf et al. 2008).

In addition to interspecific spatial patterns on the population level, we investigated the distribution of individuals. Interactions take place on the level and decisions to interact with another individual or to evade it are determined by physical recognition. Competitor and predator recognition in Old World primates can be based solely on acoustic signals (e.g. Hauser and Wrangham 1990), but lemurs additionally communicate via visual and olfactory cues. Far-reaching acoustic signals and long-lasting odors enable recognition of other individuals over greater distances than visual cues. Olfactory signals are considered of particular importance to nocturnal arboreal lemurs, as they are less dependent on sighting conditions (Klopfer 1977).

Microcebus spp. use acoustic signals for intra-group coordination, but regulate intergroup distribution also by olfactory cues (Braune et al. 2005). In the context of predator detection, mouse lemurs produce agonistic whistle calls. Highly variable in structure, those calls differ interspecifically (Braune et al. 2001), serve to increase overall attention and to prevent surprise attacks (Schülke 2001), and potentially also specifies the predator (Zimmermann et al. 2000, Rakotonirainy 2003, Scheumann et al. 2007). Even though M. 
murinus in Kirindy Forest produce alarm calls in response to predators (Fichtel 2009), they did not react with anti-predator behavior to plackbacks of alarm calls (Rahlfs \& Fichtel 2010).

Mouse lemurs need to develop recognition abilities for acoustic and visual cues through experience (Deppe 2005, Deppe \& Wright 2009, Bunkus et al. 2005), but they react innately to olfactory cues of predators (presumably based on metabolites of meat digestion: Deppe et al. 2007, Sündermann et al. 2008). Mirza uses olfactory signals in addition to a variety of vocalizations (Kappeler 2003) and C. medius scent-marks its territory and sleeping holes (Fietz 1999, 2003). In sum, Microcebus spp. can be assumed to discriminate between conspecific and heterospecific competitors and to recognize potential predators not only in their immediate vicinity.

\section{Central questions and hypotheses}

The present study aimed to assess $M$. berthae's distribution and abundance within the species' global range, and to identify determinants of ecological structure. In addition to habitat degradation and anthropogenic impact, we considered coexisting cheirogaleids as potential determinants of $M$. berthae's global population structure. Finally, we aimed to elucidate the mechanism stabilizing competitive coexistence of Microcebus spp. despite extensive niche overlap (Dammhahn \& Kappeler 2008, cf. Reimann et al. 2003).

We analyzed intra- and interspecific distribution patterns on various spatial scales, and on the population as well as on the individual level, and accounted for temporal heterogeneities. According to Schweiger et al. (1999), abundance estimates are not the most appropriate way to characterize how species perceive habitat heterogeneity as they are several steps removed from fundamental processes that generate within-population patterns over space and time (birth, death, immigration, emigration). Measures of abundance treat populations as entities and largely ignore divergent reactions of individuals or age-specific behaviors (Wolff 1999) that can fundamentally change interspecific interactions and the likelihood for exclusion (Holt \& Polis 1997). However, the explorative character of this study and the large survey area to be covered justify this phenomenological approach. 
Following major questions were addressed:

1. Is eCOLOGICAL DifFERENTIATION BETWEen MICROCEBUS SPP. REFLeCTED IN THE SPECIFIC REgIONAL DISTRIBUTION AND ABUNDANCE ACROSS HETEROGENEOUS HABITATS?

\section{1a. Is ecological specialization reflected in the regional distribution of $M$. berthae?}

As an endemic specialist with a small global distribution and the entire population located close to its range boundary, $M$. berthae is likely reliant on relatively pristine habitats and sensitive to anthropogenic disturbances

$\mathrm{H}_{1 \text { (Mb.reg): }}$

Prediction $_{1 \mathrm{~A}(\mathrm{Mb} . \mathrm{reg})}$ :

Prediction $_{1 \mathrm{~B}(\mathrm{Mb} . \mathrm{reg})}$ :

Prediction $_{1 \mathrm{C}(\mathrm{Mb} . \mathrm{reg})}$ :
M. berthae is sensitive to anthropogenic disturbances

$M$. berthae's occurrence should be restricted to non-degraded habitat M. berthae's should avoid highly frequented and exploited forest areas around villages

Anthropogenic impact on $M$. berthae is most severe during the dry season, when the forest is readily accessible

1b. Are M. murinus populations homogeneously distributed across heterogeneous habitats?

In contrast, generalist $M$. murinus that have a wide biogeographic distribution covering different forest types, should be less affected by human activities.

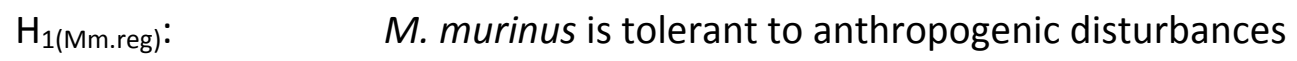

Prediction $_{1 \mathrm{~A}(\mathrm{Mm} . \mathrm{reg})}$ : $\quad$ M. murinus should be homogeneously distributed regardless of forest quality

Prediction $_{1 \mathrm{~B}(\mathrm{Mm} . \mathrm{reg})}$ : M. murinus distribution should not avoid anthropogenic environments

In order to further examine habitat suitability of degraded habitat to M. murinus, we additionally tested the hypothesis that $M$. murinus is sensitive to the reduced availability of resting holes and altered microclimate in secondary forests, which prevent females from hibernating during the dry season (Ganzhorn \& Schmid 1998).

$\mathrm{H}_{2(\mathrm{Mm} \text {.reg) }}$ : $\quad$ Females' capacity to enter energy-saving torpor during the dry season is lowered in degraded habitat

Prediction $_{2(\mathrm{Mm} . \mathrm{reg})}$ : Female dry season capture rates should be higher in degraded than in non-degraded habitat

1c. Are differential competitive strategies reflected in the local intraspecific distribution of Microcebus individuals?

On the local level, we analyzed the local intraspecific distribution of Microcebus individuals in order to reconfirm differential competitive strategies (scramble competition in M. berthae versus contest competition in M. murinus: Dammhahn \& Kappeler 2002, 2009, 2010). 
- Local intraspecific distribution of $M$. berthae individuals

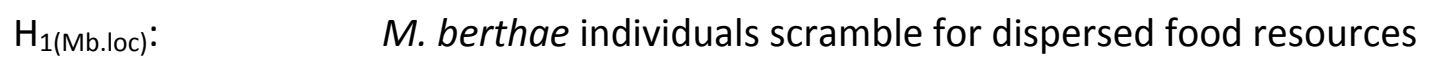

Prediction $_{1(\mathrm{Mb} . \mathrm{loc})}$ : $\quad$ M. berthae should be intraspecifically widely dispersed in space

- Local intraspecific distribution of M. murinus individuals

$\mathrm{H}_{1(\mathrm{Mm} . \mathrm{loc})}$ : $\quad$ Contest competition results from $M$. murinus individuals clustering round clumped resources

Prediction $_{1(\mathrm{Mm} . \mathrm{loc})}$ : M. murinus individuals should be intraspecifically clumped in space

\section{DO INTERSPECIFIC INTERACTIONS DETERMINE THE REGIONAL DISTRIBUTION OF MICROCEBUS SPP.?}

We tested interspecific interactions of each mouse lemur with its sister species, as well as with potential third agents. As the intensity of interspecific interactions is highly dependent on resource supply, interspecific associations should vary with forest productivity on both, the regional and local scale. In addition to spatial variability in carrying capacity, we considered intense temporal dynamics of resource supply.

2a. Is the distribution of Microcebus spp. determined by interspecific competition?

- Population-level distribution

As $M$. murinus is competitively superior to $M$. berthae, regional interspecific distribution patterns were tested for competitive exclusion on the population level.

$\mathrm{H}_{1+2(\mathrm{Mm}-\mathrm{Mb} . \mathrm{reg})}$ : $\quad$ M. murinus outcompetes and displaces $M$. berthae on a regional scale:

(1) across forest regions and (2) across transects

Predict.1+2A(Mm-Mb.reg): $M$. berthae's and $M$. murinus' regional distribution is negative complementary

Predict.2B(Mm-Mb.reg): Spatial exclusion should to be more pronounced in degraded habitat than in non-degraded habitat

Predicti.2C(Mm-Mb.reg): Spatial exclusion should be more pronounced during the dry than during the rainy season

- Interindividual distribution

Competitive interactions act on the individual level, so we tested for interindividual spatial associations on both, the regional and the local scale, and compared the relative strength of intra- and interspecific competition.

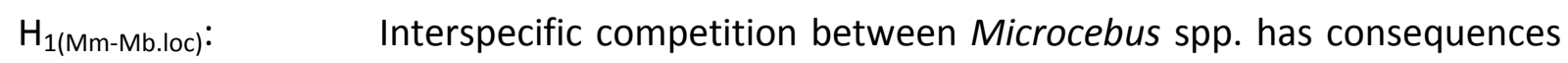
for the local distribution of individuals

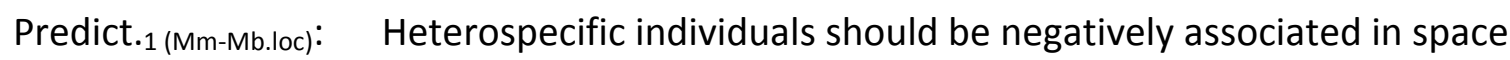




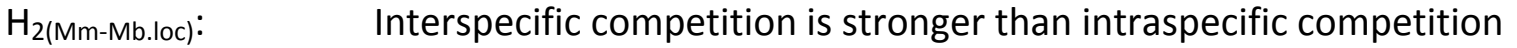

Predict.2(Mm-Mb.loc): Microcebus individuals should be found disproportionally more frequently in association with conspecifics than with heterospecifics

$\mathrm{H}_{3(\mathrm{Mm}-\mathrm{Mb} . \mathrm{loc})}$ : Interspecific competition is most intense under scarce resource supply

Predict.3 (Mm-Mb.loc): Individuals should avoid close spatial association with heterospecifics predominately in degraded habitat and during the dry season

\section{2b. Do interspecific interactions with third agents lower habitat suitability for $M$. murinus}

\section{and thereby prevent competitive exclusion of $M$. berthae?}

In order to explain coexistence despite high niche overlap, we tested for further interspecific spatial associations of Microcebus spp. with closely related, sympatric cheirogaleids. If a third agent stabilizes the coexistence of Microcebus spp., the respective species should be negatively associated in space with $M$. murinus. If $M$. berthae profits from reduced competitive pressure in consequence of the third agent's regulative impact, they should considerably overlap in space. Such contrasting interspecific associations may be reflected on the population or on the individual level and on various spatial scales. We tested interspecific interactions with $M$. coquereli and $C$. medius as potential third agents $(P$. pallescens was excluded from analyses due to pronounced ecological differentiation).

\section{M. coquereli}

Mirza coquereli overlaps with Microcebus spp. in utilized food resources, particularly during the dry season when they all rely on homopteran secretions (Kappeler 2003). Anecdotal evidence for $M$. coquereli preying upon mouse lemurs is limited to $M$. murinus (Goodman 2003, Kappeler 2003, Hart 2007, Schliehe-Diecks et al. 2010). Therefore, M. murinus should be more strongly impaired by interspecific interactions with the intraguild predator than $M$. berthae.

- Population-level distribution

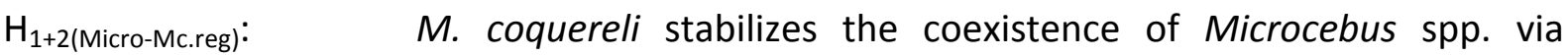
predatory interactions or intraguild predation with $M$. murinus

(1) across forest regions and (2) across transects

Predict.1+2A(Micro-Mc.reg): $M$. murinus evades $M$. coquereli as a predator or competitor, or both, resulting in negative spatial associations on a regional scale

Predict.1+2B(Micro-Mc.reg): $M$. coquereli is positively associated in space with $M$. berthae

Predict.2c(Micro-Mc.reg): Negative complementary spatial association patterns in consequence of interspecific interactions should be most distinct under scarce resource supply 
- Interindividual distribution

$\mathrm{H}_{1 \text { (Micro-Mc.loc): }}$

M. coquereli has a negative impact on $M$. murinus via predatory interactions or intraguild predation, whereas interactions of $M$. berthae with $M$. coquereli are limited to feeding competition during the dry season

Predict.1A(Micro-Mc.loc): We expect negative interspecific distribution patterns of $M$. coquereli and $M$. murinus individuals

Predict.1B(Micro-Mc.loc): Associations of $M$. coquereli individuals with those of $M$. berthae during the dry season do not need to be positive, but should be less pronounced than with $M$. murinus individuals

Predict.1C(Micro-Mc.loc): Determinative association patterns should be most prominent when resources are scarce and relax with raising productivity

\section{C. medius}

As a superior competitor, also $C$. medius has the potential to regulate the populations of $M$. murinus (Ganzhorn \& Kappeler 1996), whereas interspecific competition with M. berthae is unlikely in consequence of differential habitat utilization patterns (Schwab \& Ganzhorn 2004).

- Population-level distribution

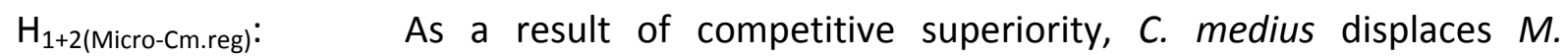
murinus; $M$. berthae profits from refuges from competition with the congener, whereas competition with $C$. medius is relaxed due to differential habitat utilization (1) across forest regions and (2) across transects

Predict.1+2A(Micro-Cm.reg): Negative spatial association of $M$. murinus and $C$. medius

Predict.1+2B(Micro-Cm.reg): C. medius should be positively associated in space with $M$. berthae Predict.1C(Micro-Cm.reg): Negative complementary spatial association patterns in consequence of interspecific interactions should be most pronounced when competition is intense, i.e. in degraded habitat $(C$. medius only active during the rainy season)

- Interindividual distribution

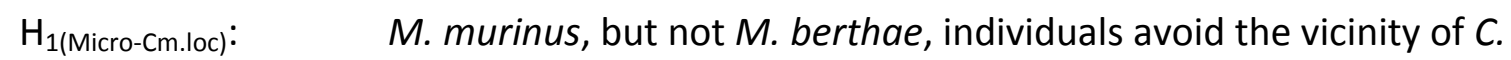
medius as a superior competitor (Schwab \& Ganzhorn 2004)

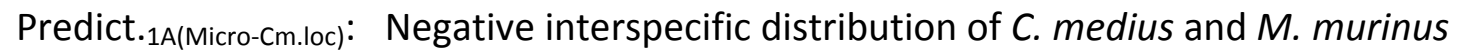
individuals

Predict.1B(Micro-Cm.loc): Positive association of $C$. medius and $M$. berthae individuals

Predict.1C(Micro-Cm.loc): Divergent associations of Microcebus and C. medius individuals should be more pronounced in degraded than in non-degraded habitat (only rainy season data) 


\section{$\underline{4 .}$ Regional community composition across heterogeneous habitats}

Finally, we explored how community structure varied with respect to anthropogenic disturbances such as habitat degradation or human frequentation. The populations of $M$. coquereli, C. medius, and P. pallescens have never been surveyed across Menabe Central, making it necessary to assess the specific regional distributions to get a glimpse on the impact of anthropogenic activities on community composition.

4a. Is the taxonomic assemblages deprived of species in consequence of anthropogenic disturbances?

$\begin{array}{ll}\mathrm{H}_{1 \text { (assembl.reg): }} & \begin{array}{l}\text { Degraded habitats do not support the entire community inhabiting } \\ \text { pristine forest }\end{array} \\ \text { Predict.1(assembl.reg): } & \begin{array}{l}\text { Species comprised in the cheirogaleid assemblage are } \\ \text { inhomogeneously distributed across degraded and non-degraded } \\ \text { habitats }\end{array} \\ \mathrm{H}_{2 \text { (assembl.reg): }} & \begin{array}{l}\text { Assemblage composition is a function of human activity, which is most } \\ \text { severe where the forest is highly frequented } \\ \text { Predict.2(assembl.reg): } \\ \text { The assemblage is deprived of one of more species in the vicinity of } \\ \text { Villages }\end{array}\end{array}$

\section{MATERIAL \& METHODS}

\section{Surveys}

In order to realistically assess specific responses to habitat alteration and threats to persistence, information on abundance and distribution of species inhabiting the forest are required (Lindenmayer et al. 2000). Patch-specific demographic data are best suited to examine consequences of anthropogenic disturbances on population and community ecology (Swihart et al. 2003). Unfortunately, these data are not easily obtained on a large spatial scale, but can be substituted by occurrence data, which are sufficient to assess population structure and tolerance to heterogeneous or fragmented habitats (Vos et al. 2001, Swihart et al. 2003). To obtain occurrence data of cheirogaleids, we established several 1-km line transects in each of the forest parts.

Systematic designs with fixed total line length and random sample size are considered superior to random sampling (Buckland et al. 2001). However, due to remoteness of some of the forest areas, transects were not evenly distributed across regions: we sampled 15 transects in Kirindy, six in Ambadira, six in the corridor connecting 
the two regions (figure 12), and four in RSA. Within the respective regions, transects were evenly distributed to the extent deemed feasible owing to low accessibility of dense dry deciduous forest (see below).

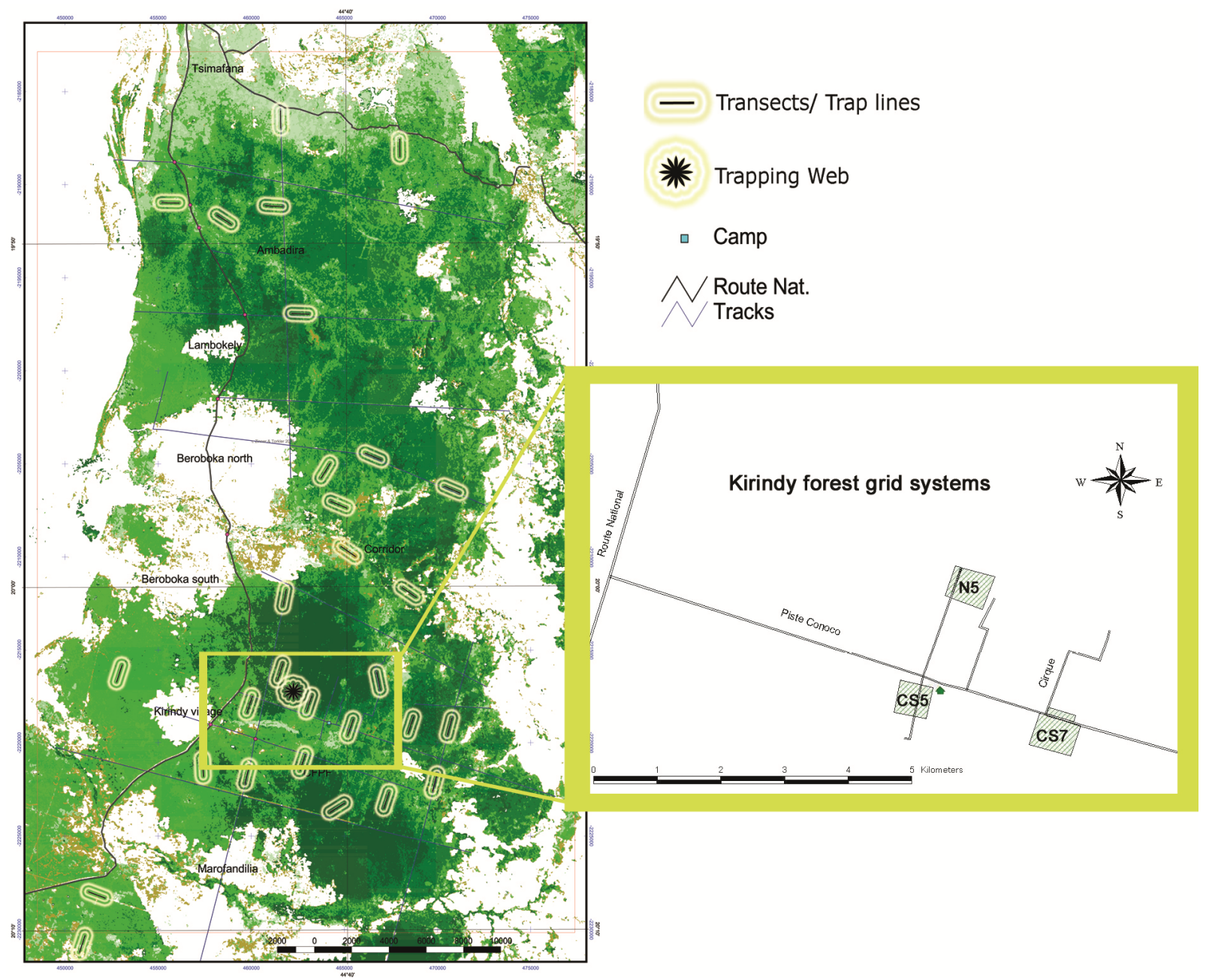

Fig. 12: Distribution of $1 \mathrm{~km}$-line transects in Ambadira $(n=6)$, the corridor $(n=6)$, and Kirindy $(n=15)$ sampled from 2003 to 2008. Only 2 of the 4 transects sampled in RSA are depicted on this map. The position of the trapping web near N5 is represented by a circle, and the map excerpt shows Kirindy forest grid systems N5, CS5, and CS7, each containing additional two $500 \mathrm{~m}$ line transects.

Three transects were situated in the grid systems N5, CS5, and CS7 (map excerpt in Figure 12), which represent long-term research areas traversed by a grid of narrow trails (every $25 \mathrm{~m}$ in north-south and east-west direction). As the grids measure $500 \mathrm{~m} \times 500 \mathrm{~m}$, transects were divided in two parts of 0.5-kilometer length and located sufficiently off each other to avoid pseudo-replication ( $175 \mathrm{~m}-275 \mathrm{~m}$ distances between the respective $500 \mathrm{~m}$ lines). We surveyed cheirogaleids over four dry $(2003,2004,2006,2007)$ and two rainy season surveys (Feb-Apr and Oct-Dec 2007). The regional distribution and abundance of all cheirogaleid species was sampled along transects via distance sampling, and we reconfirmed respective presence or absence by line trapping. 


\section{Distance Sampling}

Within each survey, 13-23 transects were sampled by distance sampling (Buckland et al. 2001). The largest part was sampled repeatedly, i.e. twice per run and over subsequent surveys, amounting to a total of 150 1-km samples (table 1).

Table 1: Distance sampling runs per transect and survey

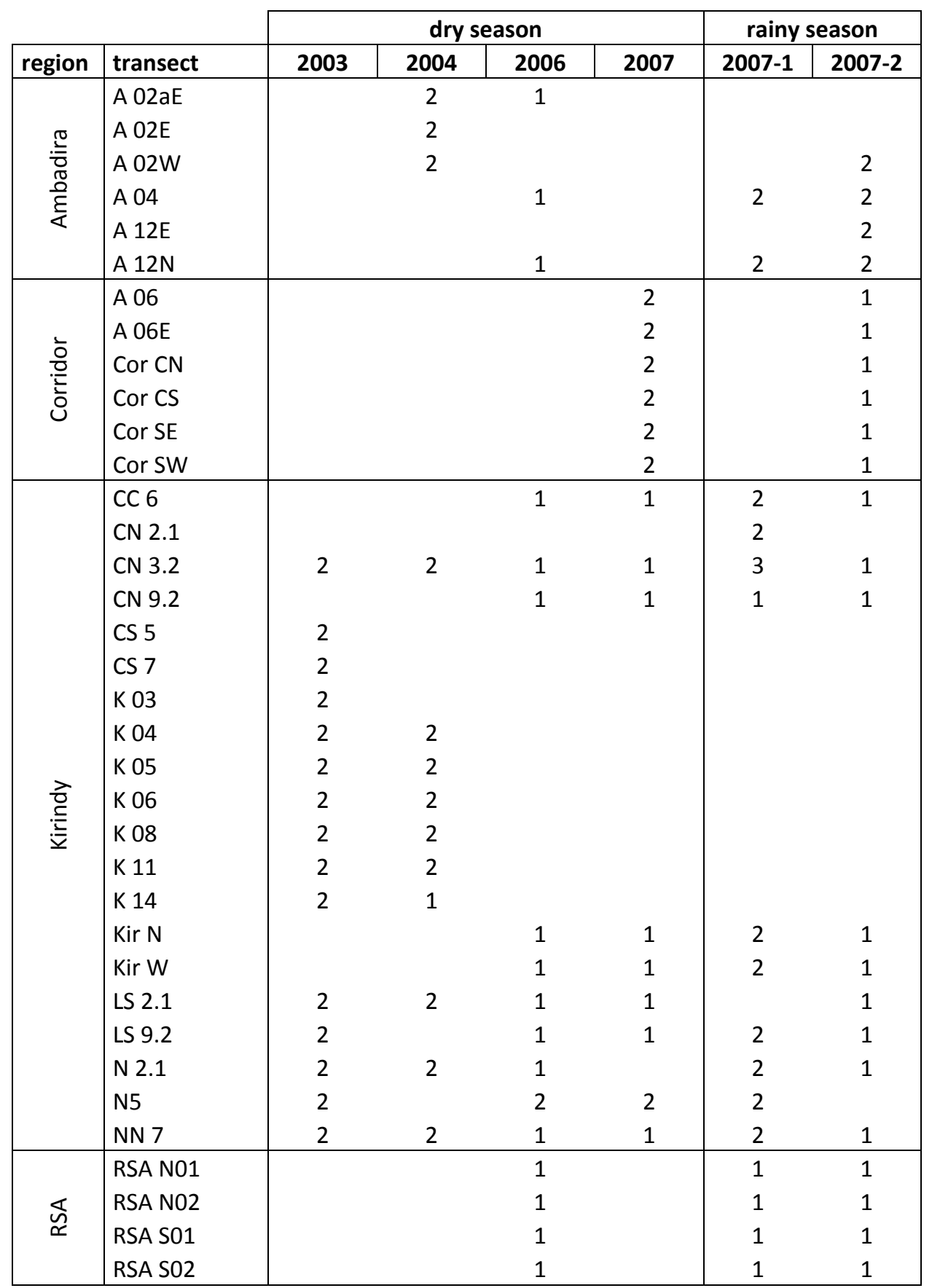

All runs were conducted between 6:00 p.m. and 8:30 p.m. in order to control for circadian variation in lemur activity. Two observers slowly walked along each transect attentively searching for lemurs. Walking pace was standardized to about $1 \mathrm{~km}$ per hour, but locally 
adjusted to habitat and sighting conditions (time spent off the trail to identify detected animals was not included in the survey time). Detected individuals were identified to the species level; when animals could not be identified with confidence, due to either dense forest or great distance of the object, sights were either recorded as 'non identified', 'Microcebus spp.' or 'greater cheirogaleids' (M. coquereli, C. medius or P. pallescens). The perpendicular distance of detected objects from the transect line was estimated independently by each of the observers to warrant reliability (Buckland et al. 2001), and if no agreement was reached, we took measurements by footsteps. Additionally, the position of detected individuals along transects was determined by a GPS-device that ensures high accuracy even under closed canopy (Garmin GPSmap 60CSx).

\section{Line Trapping}

Systematic line trapping along transects was employed in order to reconfirm species' presence/ absence, and to obtain sex-specific data. Trapping involved baiting 41 small Sherman live traps set every 25 m (type Sherman LFA: $3 \times 3.5 \times 9^{\prime \prime}$ ) and 21 larger live traps every 50m (type Sherman XLF15: $4 \times 4.5 \times 15^{\prime \prime}$ ). All 62 traps were set simultaneously on each transect for three consecutive nights. We started trapping in the dry season 2004, and subsequently trapped 11-15 transects during each survey, resulting in 63 line-trapping bouts (table 2). Trapping was limited to Ambadira, the corridor, and Kirindy, since we did not obtain the required capture permit for RSA.

\section{Trapping web}

In order examine interspecific spatial associations on a local scale, we additionally captured cheirogaleid species within a "trapping web" over three consecutive nights. Trap locations overlapped with the long-term study grid system N5 (figure 13), so that animals could be assumed to be equally familiar with traps. We trapped along four 1-km trap lines intersecting at 500 meters at an angel of $45^{\circ}$ with respect to each other. Traps were arranged in the same manner as along trap lines. We conducted trapping web captures at three different times of the year in order to get an impression of seasonal changes in the small-scale interspecific distribution of mouse lemurs (August 2006, April 2007, and December 2007). 
Table 2: Line trapping sessions per survey

\begin{tabular}{|c|c|c|c|c|c|c|}
\hline \multirow[b]{2}{*}{ region } & \multirow[b]{2}{*}{ transect } & \multicolumn{3}{|c|}{ dry season } & \multicolumn{2}{|c|}{ rainy season } \\
\hline & & 2004 & 2006 & 2007 & 2007-1 & 2007-2 \\
\hline \multirow{4}{*}{ 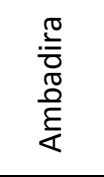 } & A 02aE & 1 & 1 & & & \\
\hline & A $02 \mathrm{~W}$ & 1 & & & & \\
\hline & A 04 & & 1 & & 1 & \\
\hline & A $12 \mathrm{~N}$ & & 1 & & 1 & \\
\hline \multirow{6}{*}{$\begin{array}{l}\frac{\overline{0}}{0} \\
\frac{0}{2} \\
\text { o }\end{array}$} & A 06 & & 1 & 1 & 1 & 1 \\
\hline & A 06E & & & 1 & & 1 \\
\hline & Cor CN & & & 1 & & 1 \\
\hline & Cor CS & & & 1 & & 1 \\
\hline & Cor SE & & & 1 & & 1 \\
\hline & Cor SW & & & 1 & & 1 \\
\hline \multirow{15}{*}{ 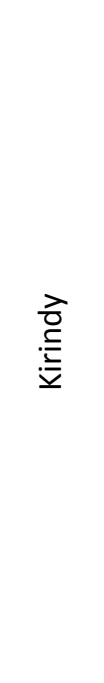 } & CC 6 & & 1 & 1 & 1 & 1 \\
\hline & CN 2.1 & & & & 1 & \\
\hline & CN 3.2 & 1 & 1 & & 1 & 1 \\
\hline & CN 9.2 & 1 & 1 & 1 & 1 & 1 \\
\hline & K 04 & 1 & & & & \\
\hline & K 05 & 1 & & & & \\
\hline & K 06 & 1 & & & & \\
\hline & K 11 & 1 & & & & \\
\hline & K 14 & 1 & & & & \\
\hline & Kir N & & 1 & 1 & 1 & 1 \\
\hline & Kir W & & 1 & & 1 & 1 \\
\hline & LS 2.1 & 1 & 1 & & & 1 \\
\hline & LS 9.2 & & 1 & 1 & 1 & 1 \\
\hline & N 2.1 & 1 & 1 & 1 & 1 & 1 \\
\hline & NN 7 & 1 & 1 & & 1 & 1 \\
\hline
\end{tabular}

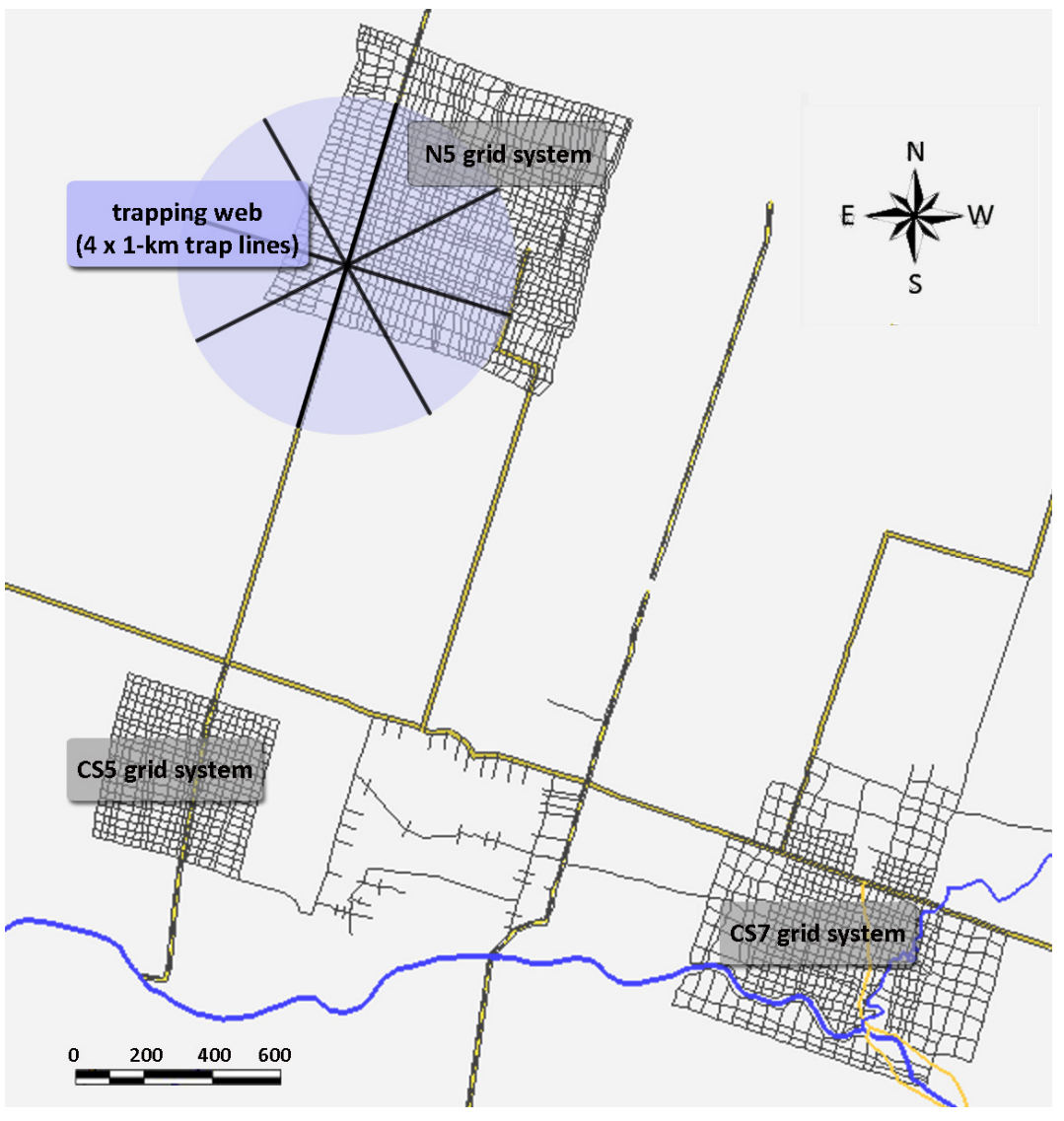

Fig. 13: Location and design of the trapping web (grid system representation by $\mathrm{M}$. Eberle) 


\section{Forest characterization}

In order to take differential habitat suitability and levels of disturbance into account, the forest surrounding each transect was roughly assessed in the field as degraded or nondegraded. Ground-based ratings were compared to forest types that have been classified based on a Landsat ETM 7 picture by Zinner \& Torkler (2005).

We exemplarily sampled forest structure along six transects (five in the corridor and one in Kirindy forest) during the late dry season 2007. Our data were complemented by the diploma work of Mevanarivo (2009), who sampled five additional line transects during early dry season 2008 (four of them in Kirindy and one in Ambadira) and resampled one in the corridor. Twenty-one sampling plots were established per transect (every 50 meters along transects), which were located 25 meters off in the forest and alternately on the left and right side of the transect line. We employed point-quarter sampling (Brower et al. 1990) in order to assess forest structure. Each sample point represents the centre of four compass directions that divide the sampling plot into quarters. For every quarter, the distance from the centre of the nearest tree to the sampling point was measured. We considered two classes of trees: mature trees (diameter at breast-height/ $\mathrm{DBH} \geq 10 \mathrm{~cm}$ ) and trees older than 10 years $(5 \mathrm{~cm} \leq \mathrm{DBH}<10 \mathrm{~cm}$; see also Ganzhorn et al. 1997, 1999, Ganzhorn \& Schmid 1998, Ganzhorn 1995, 2002, 2003). Four trees of each category were recorded at every sample point, adding up to $84(21$ plots $\times 4)$ trees per size class and transect. In addition, we recorded regenerating vegetation during the dry season 2007 by counting all trees of $1 \mathrm{~cm} \leq \mathrm{DBH}<5 \mathrm{~cm}$ within an area of $4 \mathrm{~m} \times 4 \mathrm{~m}$ around each sample point. In order to assess understory density, we systematically positioned a white cloth on four points per sampling center point in breast height and at a distance of 4 meters from the appraiser who estimated visibility in four categories (0-25\%, 25-50\%, 50-75\%, 75-100\%).

Canopy height around every sampling point was visually estimated only on the seven transects sampled in 2007. Canopy cover was estimated according to Ganzhorn (2003): at $25 \mathrm{~m}$-intervals along transects and respectively 25 meters off the trail, we recorded whether the open sky was visible through a vertically held pipe. As substantial seasonal differences in visibility between early and late dry season prohibited pooling of data, we analyzed understory density and canopy cover separately for the transects sampled in 2007 and 2008. 


\section{Data Analyses}

Statistical analyses were conducted in DISTANCE 6.0 (Thomas et al. 2010), SPSS 17.0 (Nie et al. 1975), the R-package (R Development Core Team 2005), and EcoSim v7.72 (Gotelli \& Entsminger 2010). Tests of residuals' normal distribution (Kolmogorow-Smirnow) and variance homogeneity (Levene's test) preceded parametric analyses.

\section{Multiple Covariate Distance Sampling}

\section{Test of major assumptions}

Population densities from distance data were estimated in DISTANCE 6.0 (Thomas et al. 2010). The program compensates for detection probabilities decreasing with increasing distance of the object from the observer's position, and therefore represents an advanced method to traditional strip sampling. Assuming that the detection probability right on the transect equals one, DISTANCE fits a detection function to the histogram of observed distances, which allows for compensating missed detections as well as failed identification in greater distance from the transect. As mentioned earlier, a major assumption of distance sampling is the random or systematic distribution of transects within the entire habitat, rather than exclusively along trails. Edge effects potentially corrupt density estimation (Buckland et al. 2001), but Lehman (2006b) did not find any influence of transect type on encounter rates in cheirogaleids. As sampling transversally was not feasible in the dense dry deciduous forest of Menabe Central, we needed to use former logging trails or oil exploration tracks as transects, which were abandoned and ranged between one and three meters width.

In order to achieve compliance with the model's basic assumptions we tested comparability of encounter rates among trails of various widths. For this purpose we distinguished three transect classes: "paths" represent very narrow transects (extent of less than $2 \mathrm{~m}$ ) with closed canopy, which are inaccessible by car or zebu cart ("charety") and not used to punch cattle. "Trails" resulted from abandoned logging trails that were mostly overgrown, $2-3 \mathrm{~m}$ in width, and only accessible by foot; cattle were rarely encountered on trails. Transects exceeding $3 \mathrm{~m}$ and frequented by humans with vehicles of various kinds or cattle were defined as "roads". We tested transects of various widths for differences in encounter rates per survey effort exemplarily for $M$. murinus, the only species we encountered in sufficiently high rates to allow for this statistical analysis. Encounter rates 
were calculated from total encounters per 1-km transect line and averaged over repeated runs. For each survey, we simultaneously investigated $M$. murinus encounter rates for differences in (per kilometer) between transect categories and between regions (2-factorial ANOVA). As only one region (Kirindy Forest) was sampled during the dry season 2003, encounter rates of this survey were tested for differences between transect categories by simple ANOVA.

Only individuals identified with confidence were taken into account in subsequent analyses; all others were treated as undetected objects. DISTANCE deals with non-identified objects analogously to undetected objects if the probability of identification to species level decreases with increasing distance in a similar manner as detection probability. In order to reconfirm this detection pattern we compared detection curves of identified and nonidentified objects from the dry season surveys in 2003, 2006 and 2007, as well as from early and late rainy season surveys in 2007. Non-identified objects were not expected to occur close to the transect, but rising in frequency with increasing distance from the transect. Nonidentified objects could be assigned to either "Microcebus spp." or "greater cheirogaleids" in any case. Consequently, we compared detection curves of non-identified Microcebus spp. with those of $M$. berthae and $M$. murinus during both, dry and rainy season. Detection curves of greater non-identified cheirogaleids were compared for dry and rainy season respectively to those of $M$. coquereli and $P$. pallescens, and for the rainy season to $C$. medius.

\section{Density estimation in DISTANCE}

The detection function depends on species' visual conspicuousness as well as on sighting conditions, which can differ considerably in deciduous habitats. Therefore, separate projects were run for each species. In order to account for temporal heterogeneities in distribution, which result from divergent seasonal activity patterns in some cheirogaleids ( $M$. murinus: e.g. Ganzhorn \& Schmid 1998; C. medius: e.g. Ganzhorn 1995, Dausmann et al. 2004), season was entered as a covariate in the multiple covariate distance sampling engine (MCDS: Thomas et al. 2010). Transects represented "samples" in DISTANCE, surveys were treated as "strata", and all samples across Menabe Central were embodied in the "global level". Following data exploration, distance raw data were appropriately grouped and the largest $5 \%$ of distance data discarded ("right truncation") to offset shortcomings like heaping of estimated distances on round lots and outliers detected at great distances from transect 
lines (Buckland et al. 2001). Area sizes of distinct forest regions were set to $200 \mathrm{~km}^{2}$ for Ambadira, $35 \mathrm{~km}^{2}$ for the corridor, $200 \mathrm{~km}^{2}$ for Kirindy (with the dry season 2003 survey covering only $125 \mathrm{~km}^{2}$ ), and $65 \mathrm{~km}^{2}$ for RSA.

In order to justify input of pooled encounter rates into DISTANCE, we scrutinized reliability of data from repeated runs within the same survey. For each cheirogaleid species, we analyzed variances of encounters per survey effort on repeatedly measured transects (FTest in randomized block design with transects as blocks). As pooling of repeated samples was supported (see results), transects were prolonged corresponding to sampling effort, yielding mean estimates from both samples (i.e. line transect length was prolonged to $2 \mathrm{~km}$ if sampled twice). The detection function was calculated over all samples separately for dry and rainy season by considering season as "factor covariate".

Both models provided in MCDS (half-normal and hazard-rate key functions) were tested with all available series expansions (cosine, simple polynomial and hermite polynomial). Models were chosen by visual inspection of the detection function's fit to histograms of distance data and by lowest Akaike's information criterion (AIC; Buckland et al. 2001). Population densities and encounter rates were estimated in DISTANCE by survey unit (stratum level) and for the entire survey (global level). Population densities in each of the regions represent means of stratum estimates (Thomas et al. 2010).

\section{Pooling of distance data by season}

In order to justify pooling of encounter rates per transect over surveys for subsequent analyses, we tested specific encounter rates of four consecutive surveys within Kirindy Forest (DS 2006, RS 2007-1, DS 2007, RS 2007-2) for differences between subsequent years as well as between dry and rainy season (2-factorial randomized block design (RBD) with transects as blocks). Moreover, we verified invariance of replicate samples, which resulted, first, from repeated runs within single surveys and, second, from repeated samples over subsequent surveys within the same season (F-tests in RBD with transects as blocks).

Some species are known to be partially (seasonal torpor in female $M$. murinus) or entirely inactive (hibernation in $C$. medius) during the dry season. In order to examine potential seasonal variation in other cheirogaleid populations, we tested 24 transects that were sampled at least once in each season for differences in pooled encounter rates 
between dry and rainy season (Wilcoxon signed-rank test, referred to as Wilcoxon in the following).

Aside from seasonal activity patterns, annual reproductive cycles of cheirogaleids might be reflected in encounter rates. Cheirogaleid populations show a pronounced postnuptial increase after the midpoint of the rainy season that might prohibit pooling of early and late rainy season data. In order to detect systematic differences from pre-birth to post-birth rainy season we examined encounter rates exemplarily for $M$. murinus. We tested 16 transects sampled during both, early and late rainy season, for differences in encounter rates (Wilcoxon).

Cheirogaleids' density estimation proved invariant over consecutive surveys, and were not corrupted by demographic effects (see results). Therefore, we averaged regional densities given by DISTANCE per forest area over subsequent surveys. Subsequent analyses were likewise based on average encounter rates per transect. We analyzed dry and rainy season data separately to document cheirogaleids' responses to temporal variations in food supply, and to account for seasonal differences in detection probabilities in some species (see RBD results).

\section{Forest data}

Each transect was assigned to degraded or non-degraded habitat based on ground truthing. As forest structure was recorded only along 12 transects, we could not assess habitat degradation of all transects based on habitat variables. Therefore, we tested transects assigned to non-degraded and degraded habitat for differences in habitat variables. The entire set of transects (17 in non-degraded and 19 in degraded habitat) were tested for differences in distance from the closest village (ANOVA). Seven of the transects, on which forest structure was sampled, were located within rather intact habitat and five transects were subject to anthropogenic disturbances. Forest structural data were analyzed for differences between habitat categories. We first tested mean DBH in mature trees and trees older than 10 years for differences between non-degraded and degraded habitat on all 12 transects (Mann-Whitney U-test). As a measure of forest density, we compared mean distance of the nearest tree of each class from the center point between habitat types (Mann-Whitney U-test). Moreover, canopy height and number of regenerating trees were examined based on the seven transects sampled in 2007, four of which were located in 
degraded, and three in non-degraded habitat. In the survey of Mevanarivo (2008), four transects were placed in non-degraded, and two in degraded habitat. In order to avoid seasonal bias, canopy cover and understory density were analyzed separately for each of the forest surveys, and in case of consistency data were subsequently pooled (Mann-Whitney Utest). Finally, we tested variables that significantly differed between degraded and nondegraded habitat for correlations with other forest variables (Spearman rank correlation).

\section{Regional scale: spatial population structure in cheirogaleids}

Some basic exploratory analyses provided a first impression on the significance of factors that potentially influence the distribution of cheirogaleid species. Analyses were based on encounter rates rather than on density estimates as they are less fraught with assumptions. We scrutinized the regional distribution of cheirogaleids for impacts of habitat degradation and human frequentation and considered temporal heterogeneities. In addition, we tested either mouse lemur's population structure for consequences of interspecific interactions with the congener and the greater cheirogaleids. As elaborated earlier, determinative interspecific interactions of Microcebus spp. and $P$. pallescens are not to be expected. We therefore confined analyses of Phaner's distance samples to regional population density estimates and to the description of the species' distribution in relation to anthropogenic disturbances.

\section{Test of survey design}

In order to ensure that our survey design qualifies for the analysis of factors determining lemur distribution, we analyzed the allocation of transect classes among forest regions $\left(\chi^{2}-\right.$ test) and in relation to anthropogenic pressure. Under the assumption that human impact is strongest close to villages, and declining with increasing distance from them, transect categories were tested for differences in distance from the nearest village (ANOVA). Moreover, we tested the distribution of transect categories among anthropogenically degraded and non-degraded habitat $\left(\chi^{2}\right.$-test).

\section{Regional differences in cheirogaleid encounters}

In order to describe regional distribution patterns of cheirogaleids in Menabe Central, we inspected species' distribution within the four distinct forest regions. Specific encounter 
rates were tested by season for differences between the regions (Kruskal-Wallis ANOVA). In total, 34 single transects were surveyed during dry season (Ambadira $n=5$, Corridor $n=6$, Kirindy $n=19, R S A n=4$ ), and 25 during rainy season surveys (Ambadira $n=4$, Corridor $n=6$, Kirindy $n=11, R S A n=4)$.

\section{Cheirogaleid encounters in degraded and non-degraded habitat}

In order to quantify the influence of habitat suitability on cheirogaleids' population densities, we tested specific encounter rates by season for differences between degraded and nondegraded habitat (Mann-Whitney U-test). In addition to encounter rates as a proxy for population density, we explored the proportions of transects in degraded and non-degraded habitat on which $M$. berthae and $M$. coquereli were present. Microcebus murinus encounter rates were sufficiently high to divide them into four density classes, ranging from no detection (class 0 ) to less than five individuals per kilometer (class 1 ), less than ten (class 2 ), to ten or more encounters (class 3). Cheirogaleus medius encounter rates allowed for categorizing into four classes in: absence from a transect (class 0 ), below two individuals detected per kilometer (class 1), below four (class 2), and four or more individuals (class 3 ). We analyzed 16 dry season transects in non-degraded and 18 in degraded habitat, as well as eleven non-degraded and 14 degraded forest transects surveyed within the rainy season for differences in the proportion of transects colonized $\left(\chi^{2}\right.$-test).

Moreover, we aimed to detect potential seasonal changes in lemur presence or density on transects that were surveyed in both seasons in order to recognize potential capacity tracking (cf. Roughgarden 1974). We compared the distribution of lemur populations on 13 degraded and 10 non-degraded habitat transects in a pairwise fashion between seasons (Wilcoxon). As a proxy we used (1) specific encounter rates and (2) the proportion of transects on which a species was either present or absent ( $M$. berthae, $M$. coquereli: sign test) or occurred in various density classes (M. murinus: Wilcoxon).

\section{Cheirogaleid captures in degraded and non-degraded habitat}

Density estimates based on capture rates do not reflect true population densities, as they seasonally differ in trapability, e.g. if parts of the population are hibernating or do not enter the traps (Ganzhorn \& Schmid 1998). In addition, cheirogaleids habituate to traps if frequently baited, but they can be quite timid when encountering traps for the first time. 
Our transects ranged from regularly trapped to never sampled before, and line trapping results are consequently not suited for population density estimation or interspecific comparisons of capture rates. Therefore, trapping data were employed primarily to reaffirm presence or absence of individual species and their distribution on transects in degraded and non-degraded habitat.

Specific capture rates were analyzed for sex-specific differences between degraded and non-degraded habitat in order to assess habitat suitability to Microcebus spp., $M$. coquereli, and C. medius (Mann-Whitney U-test; regardless of season). To avoid dependencies between observations, we only considered first captures of single individuals in our analyses. For Microcebus spp. and M. coquereli, tests were based on pooled data from all surveys (degraded: $n=20$; non-degraded: $n=32$ ), and on data of dry season surveys only (degraded: $n=9$; non-degraded: $n=15$ ). For $C$. medius, we analyzed capture rates of rainy season line trapping sessions (degraded: $n=11$; non-degraded: $n=17$ ), which did not yield sufficiently high samples sizes in any other species to allow for separate statistical analysis.

\section{Sex-ratio in M. murinus captures in degraded and non-degraded habitat}

Moreover, trapping data allowed for a closer examination of degraded habitat suitability to M. murinus. In order to reconfirm reduced capacity to enter energy-saving torpor in female M. murinus due to altered microclimatic conditions in secondary forest tree holes (Ganzhorn \& Schmid 1998), we tested whether dry season capture rates vary with the factors "habitat degradation" and "sex" (2-factorial ANOVA).

\section{Cheirogaleid encounters related to distance from the closest village}

In order to appraise effects of forest utilization by humans, we used the distance to the closest village as an inverse proxy. Spearman rank correlations assessed the relation of cheirogaleid encounter rates with distance of transects from the next village separately for each season (dry season $n=34$; rainy season $n=25$; table 2 ).

\section{Interspecific distribution of Microcebus populations in heterogeneous environments}

For a first impression of how interspecific competition between the mouse lemurs depends on habitat heterogeneity, we tested either species' encounter rates in degraded and nondegraded habitat and in dry and rainy season respectively for differences between transects 
with different population densities of the sister species. Microcebus berthae's encounter rates were tested for differences between transects that are colonized by $M$. murinus in the four density classes described earlier (Kruskal-Wallis ANOVA). Considerably lower population densities in $M$. berthae only afforded opportunity to test $M$. murinus encounter rates for differences between transects on which $M$. berthae was either present or absent (MannWhitney U-test).

\section{Determinants of regional mouse lemur distribution: Log-linear models}

We aimed at a comprehensive model to account for the complexity of factors that determine the regional ecological structure of coexisting Microcebus spp., including potential determinative interaction with third agents. Log-linear models were fitted to the encounter rates of Microcebus spp. in SPSS (Nie et al. 1975), which allow for appraising the relative strength of structuring factors, as well as for detecting interactions between interspecific effects and environmental variables. In order to account for seasonal differences in ecological structure, we modeled dry and rainy season data separately.

For each mouse lemur species, we fitted negative binomial distributions of detection events combined with a log-link function (overdispersion indicated that Poisson models were prone to Type I errors) to logarithmic encounter rates. Encounter rates were corrected for varying sampling effort (i.e. total transect length) entered as an offset term.

The number of transects was too low to allow for testing all potentially influential variables and factors and subsequently withdrawing those that have no effect. Therefore, explanatory variables were systematically added based on the results of our explanatory analyses. We started off with proxies for anthropogenic disturbances, the forest regions, and encounter rates of relevant coexisting cheirogaleids, to then proceed with the examination of factor interactions. As a measure of anthropogenic disturbances, we deployed the factor "habitat degradation" and the variable "distance to the next village". The low number of transects on which forest structure was sampled did not allow for including structural characteristics of the habitat. We modeled negative binomial distributions with natural loglink function, and assessed model fit based on lowest AIC. The amount of total variation in encounter rates explained by best-fitting models was quantified by the coefficient of determination pseudo- $\mathrm{R}^{2}$. 


\section{Local scale: interspecific distribution patterns on the individual level}

\section{Distance samples}

In addition to regional analyses of cheirogaleid populations, we examined our distance data for small-scale distribution patterns indicative of interspecific interactions between Microcebus individuals, as well as between each mouse lemur species and the greater cheirogaleids. If results of our regional analyses are to be attributed to interspecific interactions, the respective species pair's small scale distribution should not contradict the regional pattern. We appraised local positive or negative intra- and interspecific associations between individuals, which were quantified by the distances between detected individuals. To our knowledge, recognition distances of cheirogaleids have not been investigated so far, so that we needed to determine relevant interaction distances based on specific home ranges sizes (table 3 ). We assumed recognition to be warranted within $100 \mathrm{~m}$ distance, a value well below the additive home range radii of all intra- and interspecific pairs of individuals. Within this distance, interactions may take place that determine the distribution of individuals.

Table 3: Home range sizes in cheirogaleid species

\begin{tabular}{|l|c|c|l|}
\hline \multicolumn{1}{|c|}{ species } & max. HR size [ha] & radius [m] & \multicolumn{1}{c|}{ reference } \\
\hline M. murinus & 4 & 115 & Eberle \& Kappeler 2002 \\
\hline M. berthae & 10 & 180 & Dammhahn \& Kappeler 2005 \\
\hline C. medius & 2 & 80 & Fietz 2003 \\
\hline M. coquereli & 4 & 115 & Kappeler 2003 \\
\hline
\end{tabular}

In our intraspecific analysis, we only considered sections that generally allowed for pair formation, i.e. on which we observed more than one individual of the respective Microcebus spp. within a run. Similarly, we analyzed interspecific distances between individuals of all possible interspecific combinations of either Microcebus spp. with the respective congener, as well as with $M$. coquereli and $C$. medius on those sections, on which we detected at least one individual of either species within the same run. Using the R-package, we calculated the numbers of intra- and interspecific pairs between individuals observed during the same run within critical distance classes, and subsequently summed them up by season. To get a first impression, we explored the proportion of sections, on which we did either not observe any intra- or interspecific pairs of individuals below a distance of $100 \mathrm{~m}$, or one to three, or more than three pairs (for dry and rainy season respectively). 
We aimed to further examine the potential for interspecific exclusion between cheirogaleid species, and to recognize potential indications of a third agent's stabilizing effect on the Microcebus spp.'s coexistence. For each species combination, we calculated the numbers of intra- and interspecific pairs of individuals observed per section and season within $100 \mathrm{~m}$. Below this distance we assumed physical recognition warranted and individuals to mutually influence their spatial distribution in case of interspecific interactions. Individuals found at distances between 100 and 500m may have been engaged in interspecific interactions, but not necessarily all of them. Beyond a distance of $500 \mathrm{~m}$, individuals can be assumed not to be involved in evasive reactions as home range radii do not add up to more than $500 \mathrm{~m}$ in any species combination (table 3). From the proportion of intra- and interspecific pairs of individuals separated by more than $500 \mathrm{~m}$, we calculated the numbers of potential pairs that could have been formed on the respective section if interindividual interactions were insignificant for animal distribution.

In order to assess if interspecific exclusion is facilitated, we tested the strength of inter- vs. intraspecific competition by comparing the proportions of intra- and inter-specific pairs of mouse lemurs observed within $100 \mathrm{~m}$ distance to those expected in absence of direct interspecific interactions on the respective section (i.e. in a distance of more than $500 \mathrm{~m} ; \chi^{2}$ test). Competitors should mutually evade each other, and therefore form less pairs than would be expected in the absence of competitive interactions. If the proportion of interspecific pairs among those separated by up to $100 \mathrm{~m}$ is smaller than the proportion among long-distance pairs, we can conclude that interspecific competition is more intense than intraspecific competition. In contrast, interspecific exclusion through competition seems rather unlikely if more interspecific pairs are realized within a short distance than were expected based on the proportions of long-distance pairs, as this interindividual distribution indicates a lower level of interspecific than intraspecific competition.

In a similar manner, proportions of observed intra- and interspecific short-distance pairs of potential third agents ( $M$. coquereli and $C$. medius) and each of the mouse lemurs were compared to the proportions of intra- and interspecific pairs that would have been realized in a mutually independent distribution (corresponding to the number of pairs observed at a distance of more than 500m; $\chi^{2}$-test). In case of competition between Microcebus spp. and either of the greater cheirogaleids we interpreted distribution patterns as explained above. Interpretation of the spatial association is more difficult in the case of 
predation, as only the prey can be expected to escape, whereas the predator should approach detected prey. The distributional outcome thus depends on recognition abilities of predator and prey, and evasive reactions are expected to lead to spatial separation only if the prey's detection ability is superior to that of the predator. However, selection pressure to refine cognitive abilities should be stronger on the prey than on a generalist predator, and we therefore also expected negative spatial associations of potential intraguild-predator $M$. coquereli and mouse lemurs if they constituted prey.

Finally, we tested whether one of the mouse lemur species was "preferred" over the other by intraguild-predator $M$. coquereli or superior competitor $C$. medius, or whether Microcebus spp. are equally affected by competitive or predator-prey interactions. For this purpose, proportions of interspecific pairs of potential third agents and each of the mouse lemur species realized within $100 \mathrm{~m}$ distance were compared to the pairs observed in distances exceeding $500 \mathrm{~m}\left(\chi^{2}\right.$-test). If the number of interspecific pairs with one of the mouse lemur species fell below expected numbers, we interpreted this species as inferior competitor or preferred prey performing evasive reactions. In case realized pairs correspond to potential pairs, we considered Microcebus spp. to be equally affected by interspecific interactions with the respective third agent.

Following predictions are to be met if a third agent accounts for the coexistence of $M$. berthae and $M$. murinus. First, one mouse lemur species, but not the other, should be affected by competitive or predatory interactions with the third agent, and association may be negatively complementary. Second, determinative association patters should be most pronounced when resources are scarce or consumer density high. As we hypothesize the respective species to stabilize mouse lemur coexistence by locally mitigating competitive pressure on $M$. berthae via spatial exclusion of the superior congener, we predict individuals of $M$. coquereli, and possibly also $C$. medius, to be negatively associated only with $M$. murinus. As a third agent would lower interspecific competition between Microcebus spp. to a level allowing for coexistence, interindividual distribution patterns indicative of interspecific exclusion do not necessarily have to become apparent.

Sections with more than three short-distance pairs afforded opportunity to compare the observed distribution pattern of interindividual distances with that of a random distribution. We assumed intraspecific clumping in $M$. murinus, but not in $M$. berthae (cf. Dammhahn et al. 2009). Derived from our regional results, we predicted negative 
associations between Microcebus individuals. In case interspecific interactions stabilize the coexistence of Microcebus spp., M. murinus individuals should avoid those of $M$. coquereli in space, whereas $M$. berthae and $M$. coquereli should at least not be negatively associated. If the local interspecific patterns of Microcebus spp. and C. medius documented by Schwab \& Ganzhorn (2004) are generally valid in Menabe Central, negative associations within section strips between $C$. medius and $M$. murinus individuals are expected and positive associations between $C$. medius and $M$. berthae individuals.

In order to detect conclusive intra- and interspecific distribution patterns, we modeled random distributions of individuals and compared simulated inter-individual distances to those observed in the field. We analyzed interindividual distributions within the effective survey area, i.e. the line transect strip. The strip area corresponds to line transect length times maximum perpendicular detection distance. Line length was $1000 \mathrm{~m}$ in all transects but those located within grid systems. Our local scale analysis is therefore based on 37 dry season samples (31 1-km transects and six 500m-sections), and 26 rainy season samples (24 1-km transects and two 500m-sections), which are referred to as sections in the following.

Positions of detected animals were defined by the perpendicular distance from the section line $(x)$ and the position along it $(y)$. We calculated interindividual distances between individuals observed within a run (s. table 1), for intra- and interspecific pairs respectively. In order to detect non-random spatial patterns between observed individuals, we simulated random distributions of individuals. We then compared the distribution of observed and simulated interindividual distances below $100 \mathrm{~m}$ ( $\chi^{2}$-test) for each section, on which more than three of such short-distance pairs were observed after pooling by section and season.

Detection events in distance sampling correspond to a discrete probability distribution that expresses the probability of detections occurring within fixed periods of time with mean rate $\lambda$, but independently of the last event. For each run, we first compensated the number of observed animals for missed detections by applying the respective detection functions for dry or rainy season from DISTANCE 6.0 (Thomas et al. 2010), which estimates the number of individuals that were actually present. Under the assumption that the detection function for each season is invariant, we calculated densities of occurrence for dry $\left(\lambda_{D S}\right)$ and rainy $\left(\lambda_{R S}\right)$ season respectively. This corresponds to a mean 
rate of presence, rather than to the detection rate, and therefore corresponds to actual densities (individuals per unit area).

Second, for each species we generated the number of individuals present per run in 1000 simulations according to a Poisson process, with respective occurrence rates $\lambda_{D S}$ and $\lambda_{\text {RS }}$. Third, for each simulation, we distributed the estimated number of individuals at random within the section strip based on a spatial Poisson process. Given the simulated number and positions of all individuals present, we, fourth, simulated a survey that mirrors the distance sampling process in order to determine which individuals from the generated sample would have been detected. For this purpose, we determined the distance of each individual from the section line, for which the probability of detection ( $p$ ) is given by the DISTANCE detection functions. Detections of each individual were determined as successful or missed by generating binary random numbers with probability $p /(1-p)$, yielding a set of detected individuals in the generated sample of present individuals.

In order to quantify positive or negative spatial associations, we calculated interindividual distances between animals in observed and simulated samples, respectively. Distance distributions were analyzed intra- and interspecifically if below a critical distance of $100 \mathrm{~m}$, which was considered short enough to make interindividual interactions in all species combinations likely. The observed distribution of inter-individual distances below $100 \mathrm{~m}$ on single sections were pooled by season and compared to the distribution of the respective distances in samples of randomly distributed individuals (Kolmogorov-Smirnov goodness-offit test).

\section{Trapping web}

The trapping web provided valuable data on interspecific spatial associations on a limited spatial scale over three consecutive times of the year (dry season 2006, transition dry to rainy season 2007, rainy season 2007). Positions of captured animals of either species were depicted graphically and tested for non-random interspecific distribution patterns employing the co-occurrence module of EcoSim v7.72 (Gotelli \& Entsminger 2010). A presence-absence matrix of first captures, representing checkerboard pairs sensu Diamond (1975), was tested against the null hypothesis of random community assemblage. The appropriate metric for matrices with two species is the C-score index, which measures species segregation without 
requiring perfect checkerboards (Stone \& Roberts 1990). It quantifies the average number of "checkerboard units" (CU) between all possible pairs of species as

$$
\mathrm{CU}=\left(\mathrm{r}_{\mathrm{i}}-\mathrm{S}\right)\left(\mathrm{r}_{\mathrm{j}}-\mathrm{S}\right)
$$

where $\quad$ S: number of shared sites (= columns)

$r_{i}$ : row total for species $i$

$r_{j}$ : row total for species $j$

In a competitively structured community, the observed C-score should exceed the one expected in random assemblages, which resulted from 5000 simulations. As Microcebus spp. differ in population density, and therefore in specific trapping probabilities, we proportionally accounted for their respective abundance by matching the rank order of species in the null assemblage to the one in the original matrix. Observed differences of various trap locations in suitability were reflected by setting specific capture probabilities proportional to the actual number of individuals captured at the respective location. Those settings were preferred over "fixed sum" constraints, which are recommended due to lowest risk of Type I errors (Gotelli 2000, Gotelli \& Entsminger 2010). Proportional representation of differences in species abundance and local habitat heterogeneities are more realistic than the assumption of constant trapping rates per species and trap location. We included trap locations with no captures in the original matrices and retained degenerate matrices in simulations (matrices with empty rows or columns; Gotelli \& Entsminger 2010).

\section{RESULTS}

\section{Survey results}

We documented a gradient in regional abundance of $M$. berthae across forest regions: we encountered the species at highest rates in Ambadira, lower densities in the corridor and Kirindy Forest, and the species was virtually absent from the Réserve Spécial d'Andranomena (with only a single detection). The numbers of detections further analyses are based on are given in table 4.

Table 4: Number of cheirogaleids detected by distance sampling on line transects

\begin{tabular}{|l|l|l|l|l|l|}
\cline { 2 - 5 } \multicolumn{1}{c|}{} & M. berthae & M. murinus & M. coquereli & C. medius & P. pallescens \\
\hline n (total detections) & 109 & 576 & 98 & 163 & 155 \\
n (dry season) & 69 & 347 & 58 & - & 110 \\
n (rainy season) & 40 & 229 & 40 & 163 & 45 \\
\hline
\end{tabular}


Moreover, the species occurred only on a fraction of transects sampled. We encountered $M$. berthae only on $50.0 \%$ of transects sampled during dry season surveys (17 of 34), and on $40.0 \%$ sampled in the rainy seasons (10 of 25 transects). Proportions of transects colonized by $M$. berthae during dry and rainy season respectively, as determined by distance sampling, are given in figures $14 \mathrm{a}$ and $14 \mathrm{~b}$. Transects, on which we detected only a single individual over the whole study time, are separated from transects with repeated encounters within the graphs.
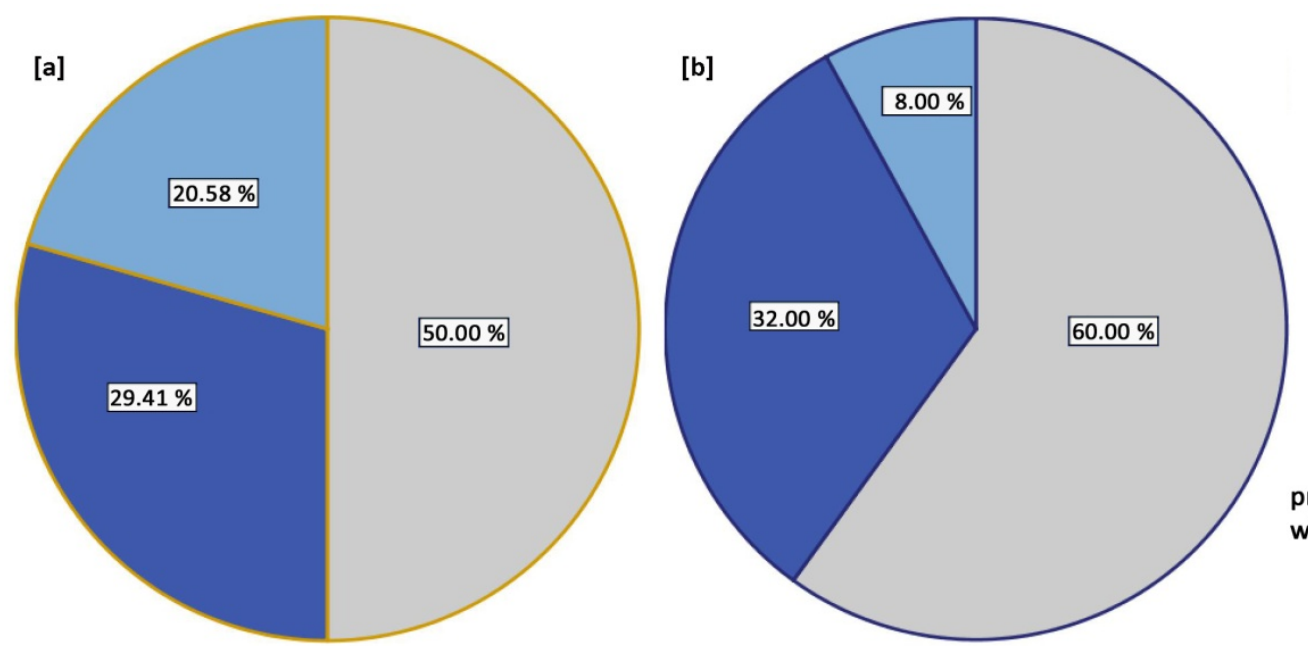

proportion of transects with $\square>1$ individual 1 individual absence

Fig. 14: Proportion of transects colonized by $M$. berthae in [a] dry $(n=34)$ and [b] rainy season $(n=25)$ surveys based on distance sampling

In contrast, $M$. murinus individuals were detected on the majority of transects, on $85.3 \%$ of dry season (29 of 34 transects) and on $88.0 \%$ of rainy season distance samples (22 of 25 transects). The proportion of transects on which M. murinus was sighted are given in figures $15 \mathrm{a}$ and $15 \mathrm{~b}$.
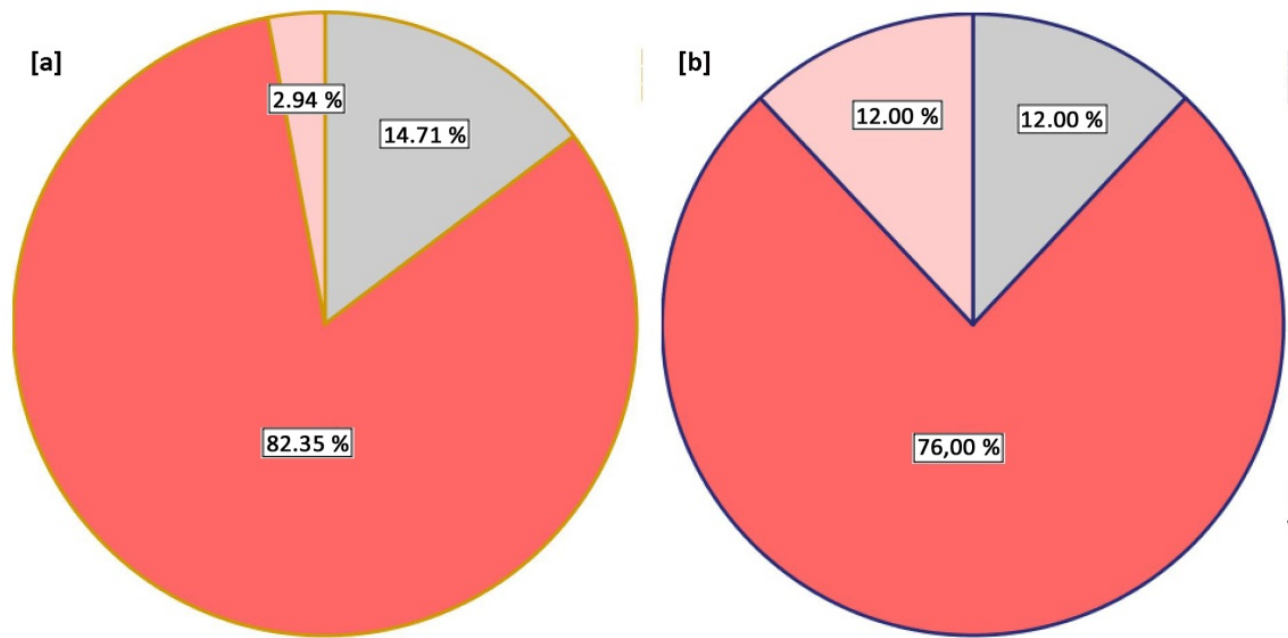

proportion of transects with $\square>1$ individual 1 individual absence

Fig. 15: Proportion of transects colonized by M. murinus in [a] dry $(n=34)$ and [b] rainy season $(n=25)$ surveys based on distance sampling 
The numbers of individuals first captured on trap lines are listed by season in table $5 \mathrm{a}$, and those of the trapping web in table $5 b$.

Table 5a: Number of cheirogaleid first captures on trap lines

\begin{tabular}{|l|l|l|l|l|}
\cline { 2 - 5 } \multicolumn{1}{c|}{} & M. berthae & M. murinus & M. coquereli & C. medius \\
\hline n (1st captures) & 52 & 168 & 11 & 57 \\
n (1st captures dry season) & 49 & 107 & 9 & - \\
n (1st captures rainy season) & 3 & 61 & 2 & 57 \\
\hline
\end{tabular}

Table 5b: Number of cheirogaleid first captures within the trapping web

\begin{tabular}{|l|l|l|l|l|}
\cline { 2 - 5 } \multicolumn{1}{c|}{} & M. berthae & M. murinus & M. coquereli & C. medius \\
\hline n (1st captures Aug 2006) & 18 & 34 & 2 & - \\
n (1st captures Apr 2007) & 10 & 45 & 3 & 1 \\
n (1st captures Dec 2007) & 5 & 61 & 0 & 0 \\
\hline
\end{tabular}

A schematic representation of Microcebus spp.'s regional distribution integrating both, distance sampling and line trapping, is presented in figure 16. A corresponding map showing the regional distribution of $M$. coquereli, $P$. pallescens, and $C$. medius is given in figure 75 in appendix. 


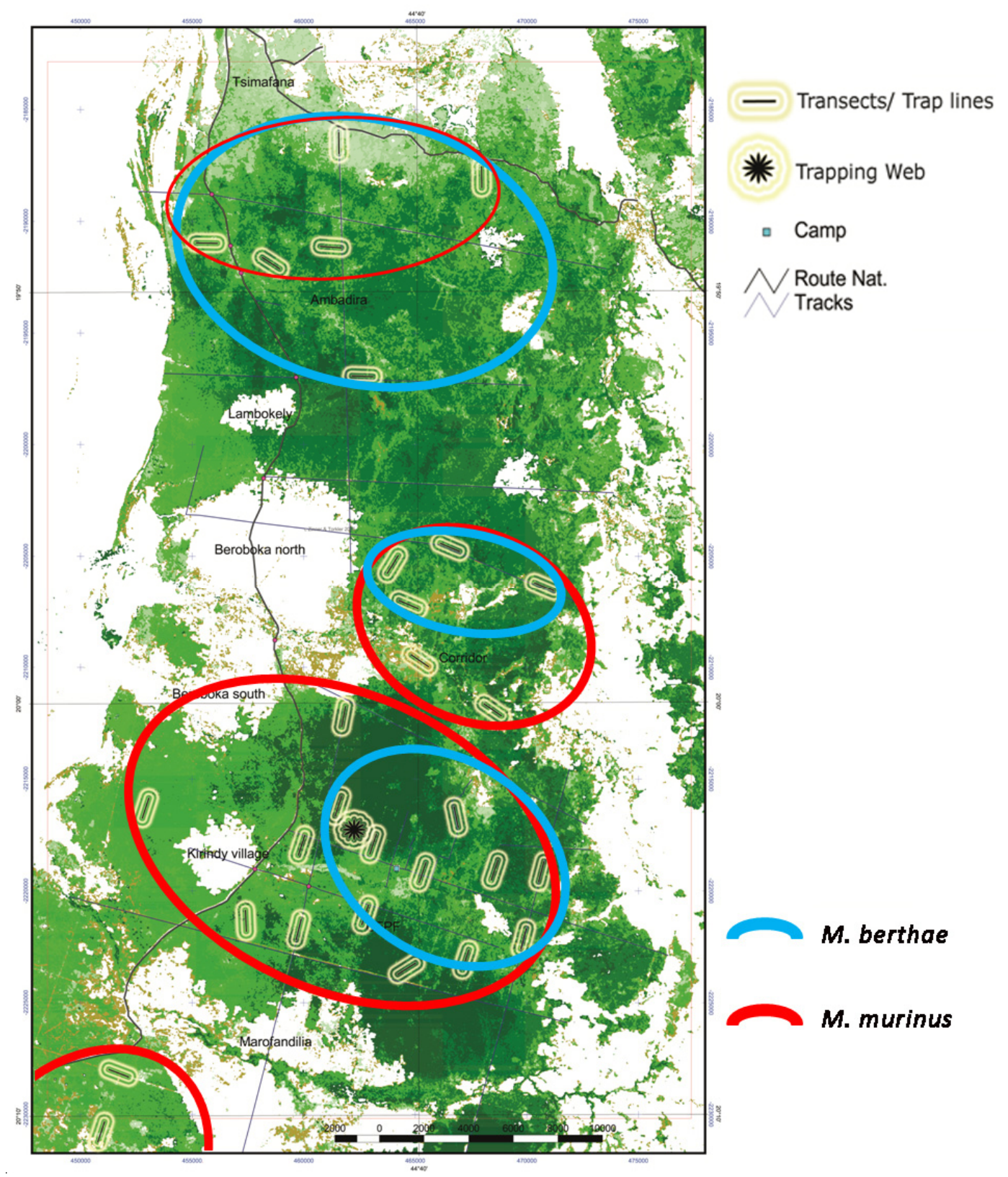

Fig. 16: Regional distributions of $M$. berthae (blue) and $M$. murinus (red), based on distance sampling and line trapping

\section{Multiple Covariate Distance Sampling}

\section{Tests of major assumptions}

Trails of different width used as transects did not cause any systematic bias in detections. No difference in $M$. murinus encounter rates between transect categories emerged in Kirindy forest during the dry season 2003 (DS 2003: $F(2,14)=0.167, p=0.848)$. Correspondingly, there were no significant main effects of transect width in any of the subsequent surveys when simultaneously considering transect category and forest region (table 13 in appendix). During dry season surveys 2006 and 2007 encounter rates of M. murinus differed between 
regions (DS 2006: $F(2,11)=4.631, d f=2, p=0.035$; DS 2007: $F(2,13)=4.802, p=0.027)$. Considerably more detections in RSA in both seasons account for this difference, which were complemented by hardly any detections in Ambadira during the dry season 2006 (figure 17a, b). Interactions between transect category and forest region were not significant in any of the surveys (table 13 in appendix).
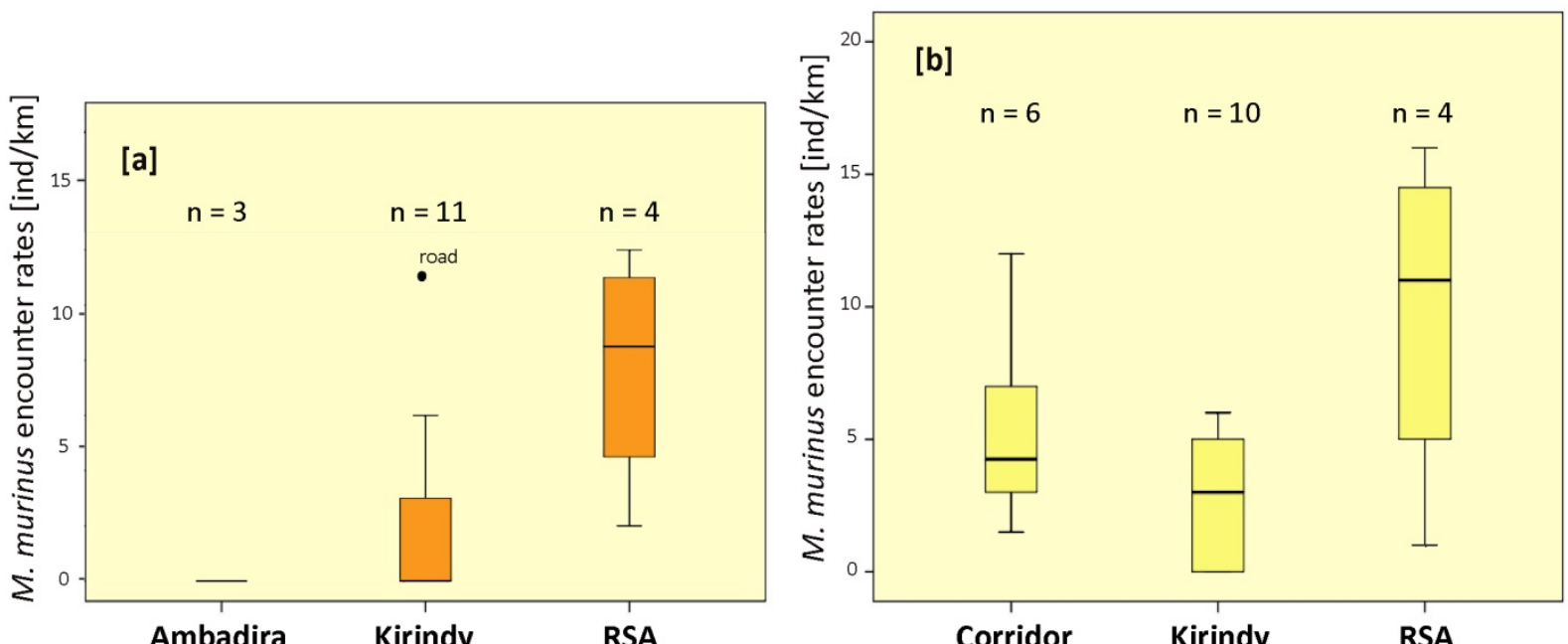

Fig. 17: Representation of regional differences in M. murinus encounter rates in [a] dry season 2006 and [b] dry season 2007

Non-identified objects did not hamper DISTANCE analyses, as the shape of respective detection curves allowed compensating failed identifications in a similar manner as missed detections. The probability of encountering an object that could not be identified to the species level equaled zero close to the transect line. During dry season surveys, detections of non-identified Microcebus individuals peaked between five and seven meters, and decreased beyond that in a similar manner as that of identified Microcebus spp. (figures 18a, b). Consequently, non-identified objects did not occur in the first distance class after grouping (see below) and DISTANCE can compensate failed identifications in a similar manner to missed detections. 

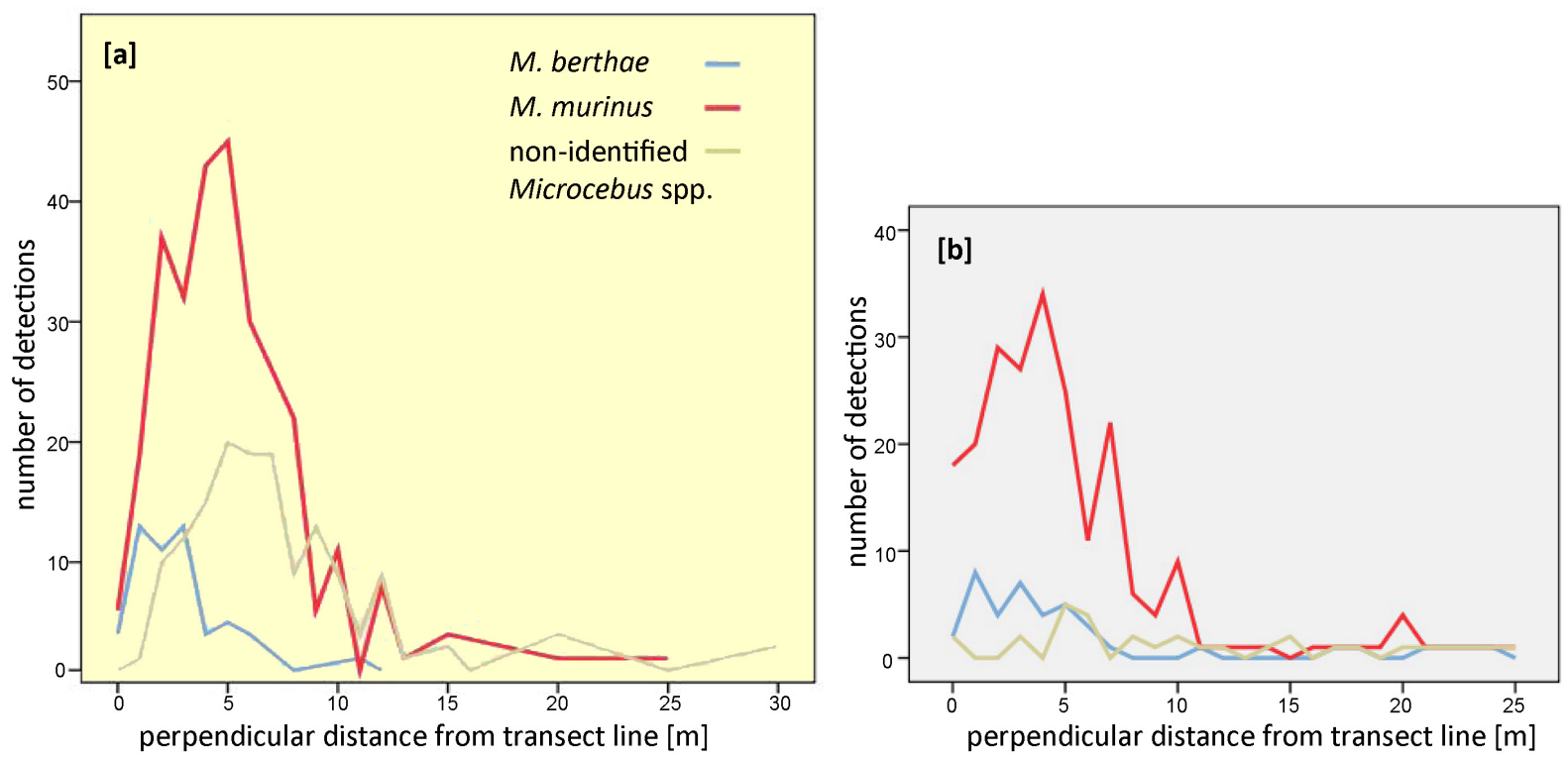

Fig. 18: Detection curves of identified and non-identified Microcebus spp. during [a] dry and [b] rainy season (dry seasons 2003, 06, 07: M. berthae $n=58, M$. murinus $n=296$, non-identified Microcebus $n=156$; rainy seasons 2007-1 and 2007-2: M. berthae $n=34, M$. murinus $n=215$, non-identified Microcebus $n=24$ )

Likewise, detections of other non-identified cheirogaleids from dry season surveys were negligible up to eight meters, then showing two peaks (5-8 $\mathrm{m}$ and $16 \mathrm{~m}$ ) before decreasing to low levels again. During rainy season surveys, detections of non-identified individuals were generally rarer, with slighter peaks somewhat closer to the transect line than in the dry season and with a steeper decline of detections with increasing perpendicular distance (figures 19a, b).
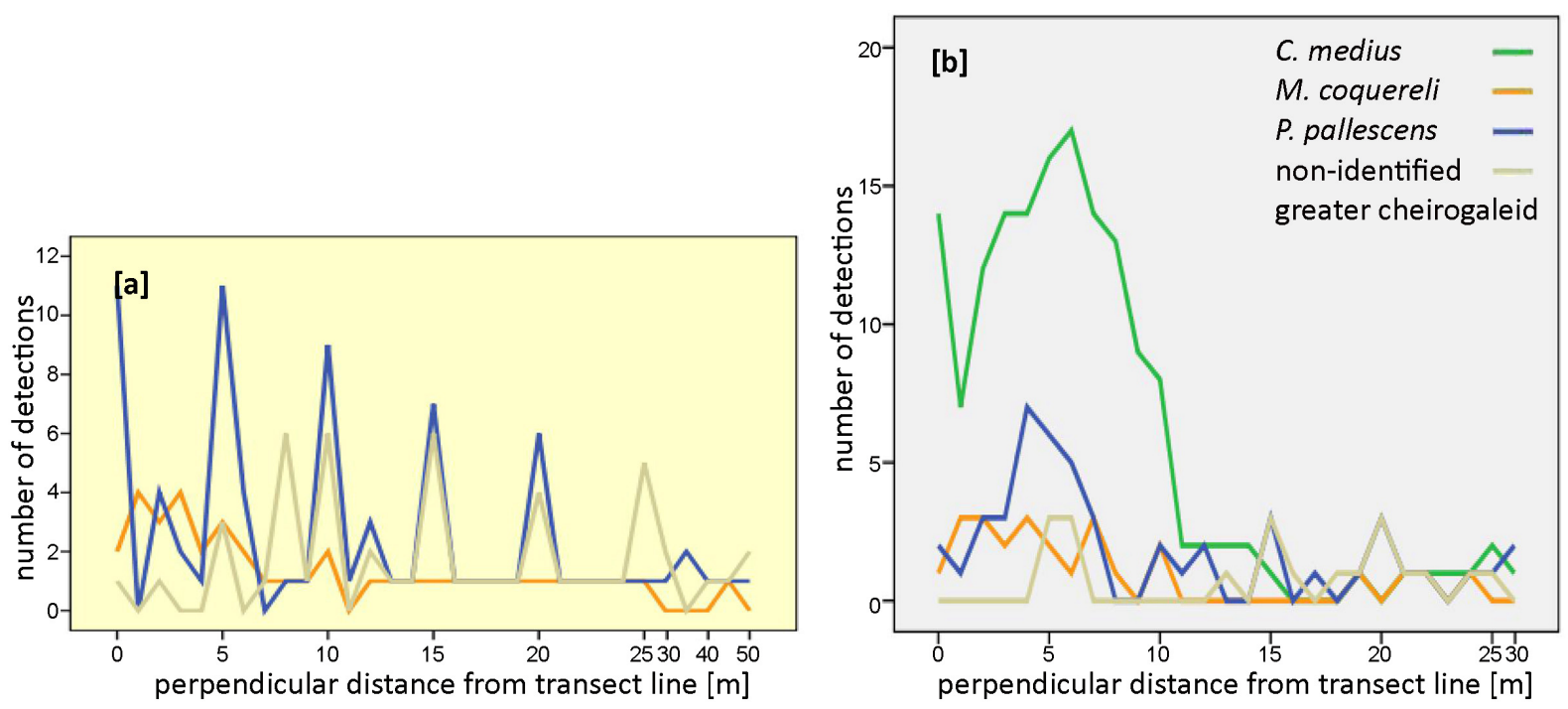

Fig. 19: Detection curves of identified and N.I. greater cheirogaleids during [a] dry and [b] rainy season surveys (DS 2003, 06, 07: M. coquereli $n=28$, $P$. pallescens $n=66$, non-identified greater cheirogaleid $n=40$; RS 2007-1 and 2007-2: $C$. medius $n=151, M$. coquereli $n=21$, $P$. pallescens $n=45$, N.I. greater cheirogaleid $n=16$ )

Shortcomings of distance data were, first, detection events not being highest right on the transect line (owing to not having sampled the forest transversally) and, second, heaping of 
detections on round lots. Appropriate grouping and right truncation effectively patched shortcomings of data, so that detection curves of identified objects conformed to the shape criterion, one of the major assumptions of distance sampling (Buckland et al. 2001; figures 20-24).

Transect-specific encounter rates proved reliable as a proxy of estimates of cheirogaleid abundances. Variances in encounter rates resulting from repeated runs within single surveys did not significantly differ in any species (tables 14 in appendix). Specific encounter rates neither differed between replicate surveys within the same season (F-Test in RBD: table 15 in appendix). Encounter rates within Kirindy forest over four subsequent surveys did not differ in any species from one year to another. Dry season encounter rates significantly exceeded those of rainy season surveys only in M. coquereli (2-factorial RBD: table 16 in appendix; $C$. medius omitted due to hibernation in the dry season). Encounter rates of the late rainy season did not exceed those of the preceding pre-birth period in any species, with merely a slight non-significant post-nuptial increase in M. murinus (Wilcoxon Z= -1.649, $n=16, p=0.099 ;$ table 17 in appendix).

\section{Density estimation in DISTANCE}

Our distance samples therefore allowed for pooling data of repeated runs by survey for subsequent analysis in DISTANCE. Sample sizes in all species exceeded the 60 to 80 detection events required for density estimation in DISTANCE (Thomas et al. 2009) and allowed for a good fit of detection functions.

Complying with impaired sighting conditions due to increased foliage, rainy season detection probabilities were generally lower than in the dry season and declined more rapidly with increasing distance from the transect line (figures 20 to 24). Best fit of the hazard-rate key function to specific distance data was affirmed by lowest AIC for the two species of Microcebus as well as for $C$. medius. Even though having a slightly higher AIC value than halfnormal keys, hazard-rate key functions were favored likewise for $M$. coquereli and $P$. pallescens. The hazard-rate model is a derived model, in contrast to models of proposed shapes, and has been shown to provide a good fit of the detection function to genuinely spiked data (Buckland et al. 2001; cp. figures 18 and 19). None of the series expansions improved model fit in any species. 
Parameter values and maximum sighting distance after truncation are given in table 6, detection functions and underlying sample sizes after truncation are represented for each species in figures 20 to 24.

Hazard-rate key function: $\quad 1-\exp \left(-\left(\frac{x}{\sigma(z)}\right)^{-b}\right)$

$x \quad$ distance from transect line

b power parameter

$\sigma \quad$ scale parameter, controlling the "width" of the detection function, is modeled as an exponential function of the covariates: $\sigma(z)=\beta_{0} * \exp \left(\beta_{1} z_{1}+\beta_{2} z_{2}+\beta_{3} z_{3}\right)$

$\beta_{0} \quad$ intercept of the scale parameter $\sigma$

z covariates

$\beta \quad$ covariate parameters

Sampled only during the rainy season, C. medius' distance function only contains two covariate parameters, therefore the scale parameter was modeled as

$$
\sigma(z)=\beta_{0} * \exp \left(\beta_{1} z_{1}+\beta_{2} z_{2}\right)
$$

Table 6: Maximum sighting distances and parameter values of distance sampling data

\begin{tabular}{|l|l|l|l|l|l|}
\cline { 2 - 6 } \multicolumn{1}{c|}{} & M. berthae & M. murinus & M. coquereli & C. medius & P. pallescens \\
\hline w [m] & 11 & 10 & 13 & 13 & 23 \\
$\boldsymbol{\sigma}$ & 3.618 & 4.976 & 5.941 & 8.270 & 9.048 \\
b & 2.519 & 4.361 & 2.752 & 7.263 & 4.652 \\
$\mathbf{z}$ & 0.02974 & 0.3968 & 0.001037 & - & 0.3867 \\
\hline
\end{tabular}




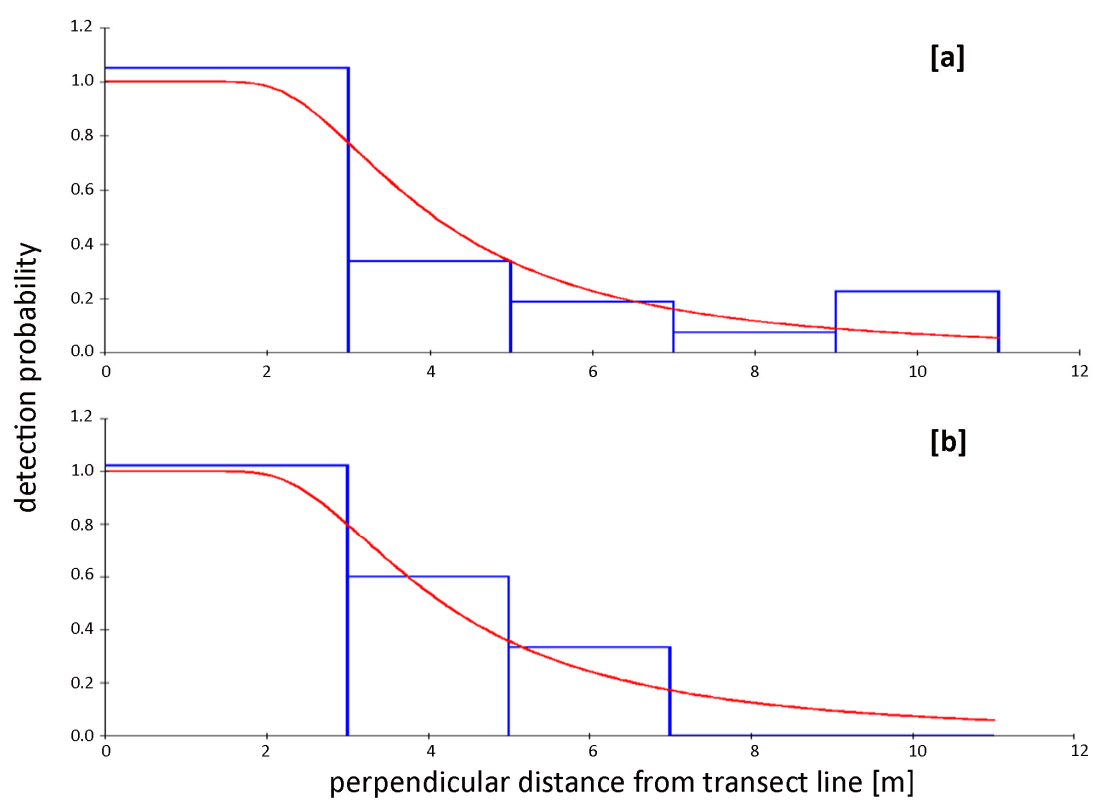

Fig. 20: Microcebus berthae distance data after truncation (blue; $n=101$ ) and detection function fitted in DISTANCE (red) in [a] dry and [b] rainy season surveys

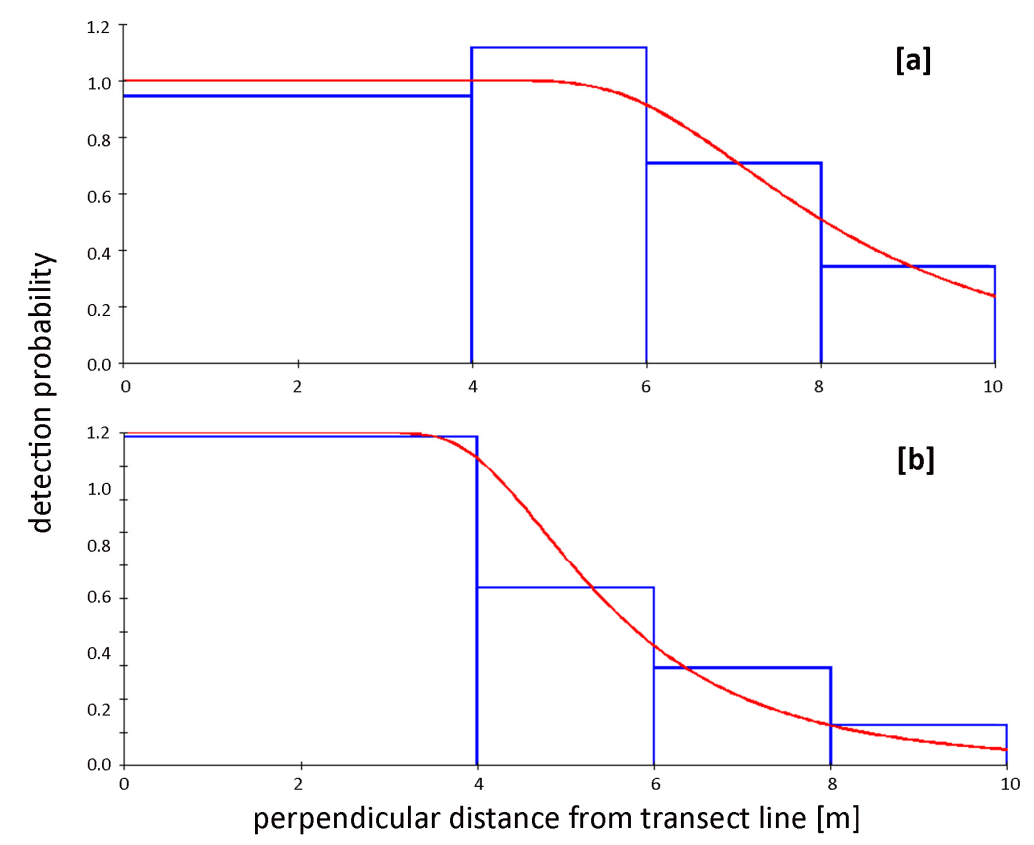

Fig. 21: Microcebus murinus distance data after truncation (blue; $n=526$ ) and detection function fitted in DISTANCE (red) in [a] dry and [b] rainy season surveys 


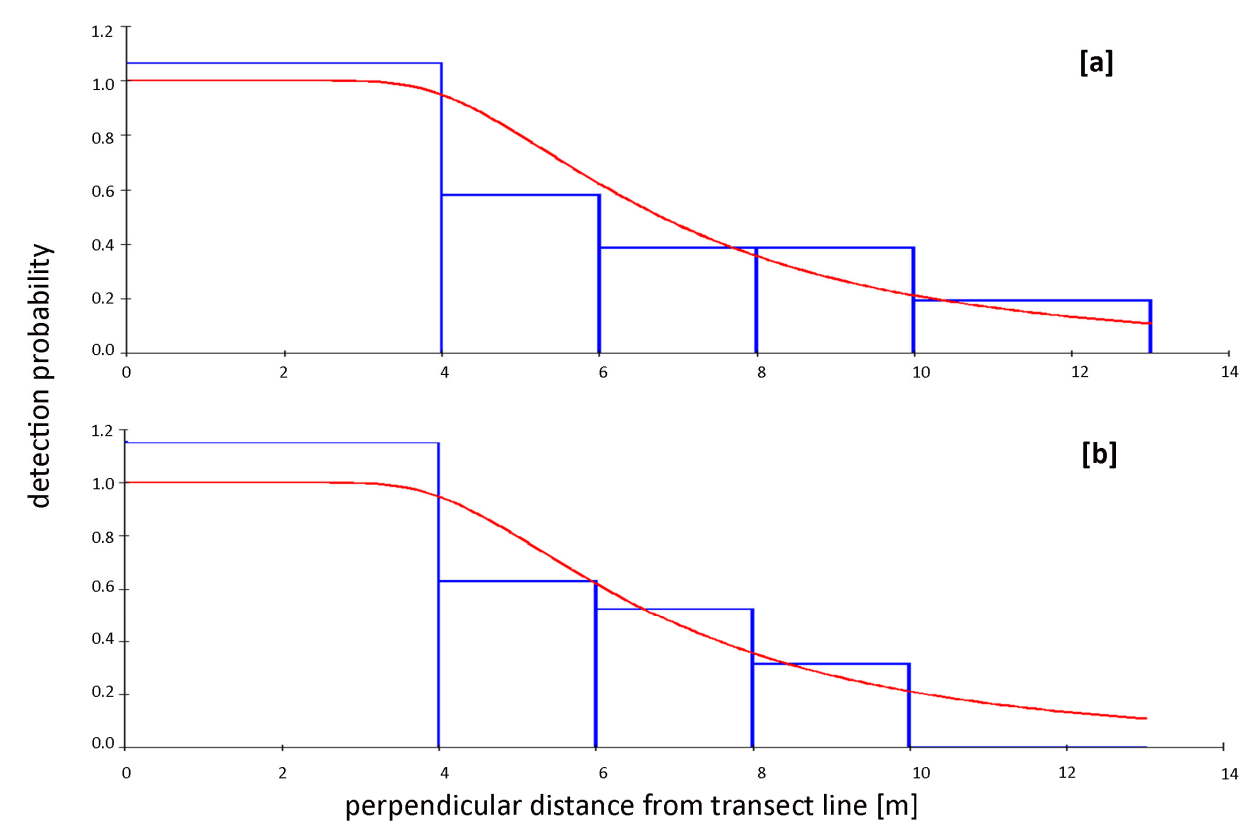

Fig. 22: Mirza coquereli distance data after truncation (blue; $n=75$ ) and detection function fitted in DISTANCE (red) in [a] dry and [b] rainy season surveys

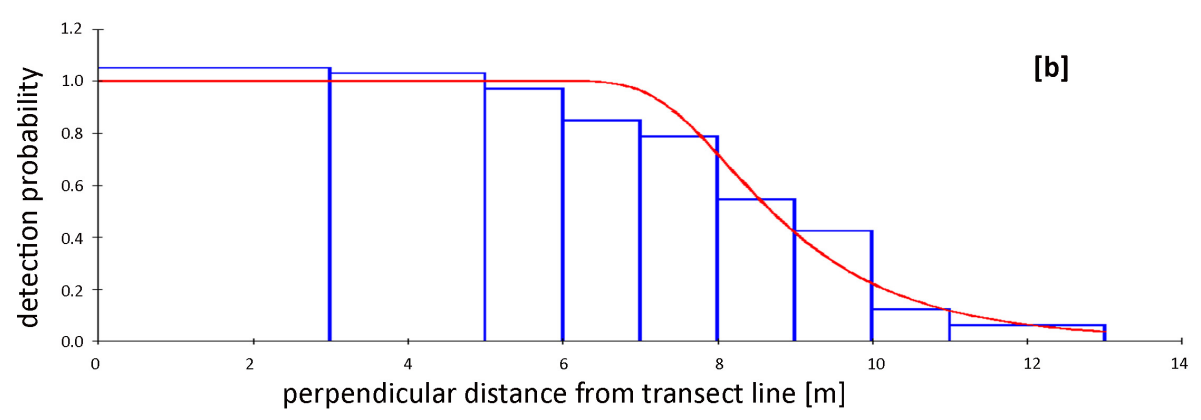

Fig. 23: Cheirogaleus medius distance data after truncation (blue; $n=149$ ) and detection function fitted in DISTANCE (red); rainy season only

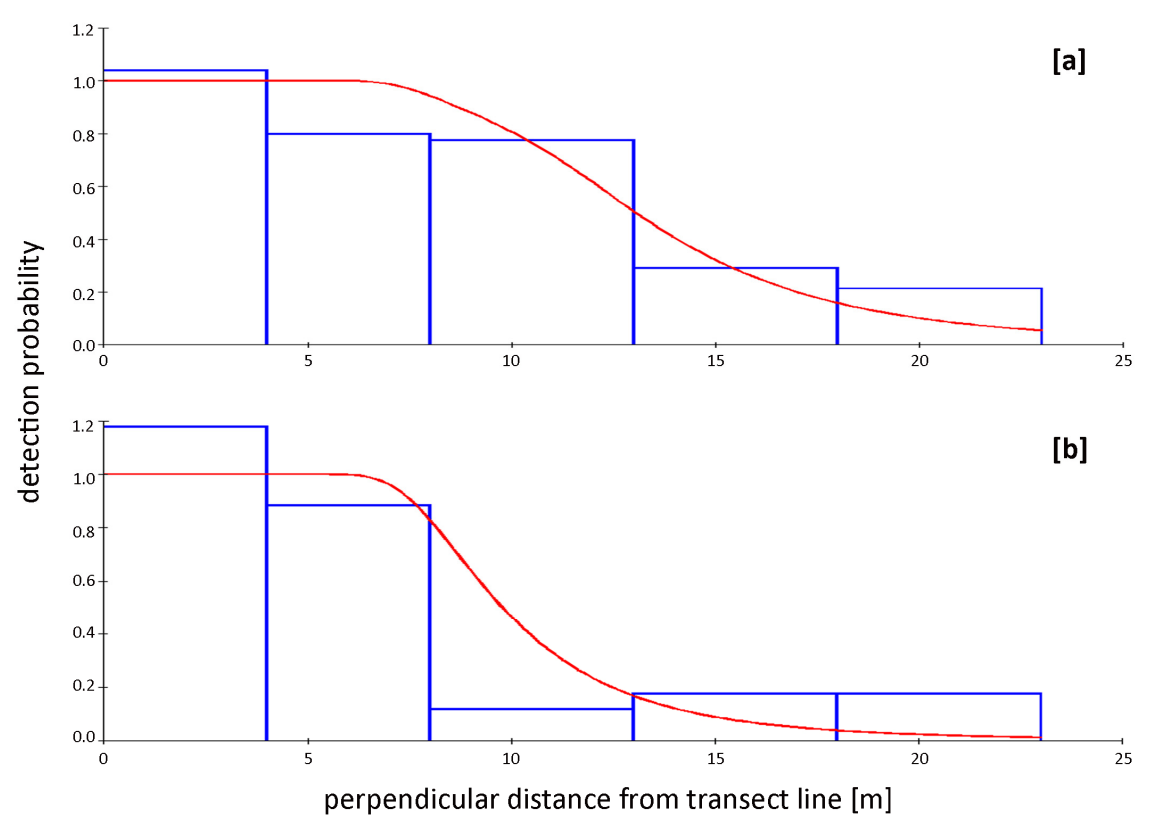

Fig. 24: Phaner pallescens distance data after truncation (blue; $n=142$ ) and detection function fitted in DISTANCE (red) in [a] dry and [b] rainy season surveys 
Population densities of $M$. berthae across Menabe Central, i.e. across the species' substantial global range, was estimated by DISTANCE to less than half the densities of $M$. murinus in that minor fraction of its biogeographic range (table 7). The only other species with regional densities exceeding 100 individuals per $\mathrm{km}^{2}$ was $C$. medius, whereas $M$. coquereli and $P$. pallescens occurred in even lower densities than $M$. berthae.

Table 7: Global density of cheirogaleids estimated in DISTANCE

\begin{tabular}{|c|c|c|c|c|c|c|c|c|c|c|}
\cline { 2 - 11 } \multicolumn{1}{c|}{} & \multicolumn{2}{c|}{ M. berthae } & \multicolumn{2}{c|}{ M. murinus } & \multicolumn{2}{c|}{ M. coquereli } & \multicolumn{2}{c|}{ C. medius } & \multicolumn{2}{c|}{ P. pallescens } \\
\cline { 2 - 11 } & ind./ km & $\% \mathrm{CV}$ & ind./ km & $\% \mathrm{CV}$ & ind./ km & $\% \mathrm{CV}$ & ind./ km & $\% \mathrm{CV}$ & ind./ km & $\% \mathrm{CV}$ \\
\hline $\begin{array}{c}\text { Menabe } \\
\text { Central }\end{array}$ & 80.334 & 39.23 & 182.47 & 11.09 & 57.814 & 24.07 & 139.97 & 13.83 & 27.562 & 19.35 \\
\hline
\end{tabular}

For a rough impression of cheirogaleids' regional distribution, we calculated mean population densities per forest part from survey-wise estimates given by DISTANCE and attained density estimates for each of the forest regions (pooled within seasons over repeated samples in table 8; underlying densities per survey yielded by DISTANCE are given in table 18 in appendix).

Table 8: Cheirogaleids' population densities by forest region [ind./ $\mathrm{km}^{2}$ ]

\begin{tabular}{|c|c|c|c|c|c|c|}
\cline { 3 - 7 } \multicolumn{2}{l|}{} & $\begin{array}{c}\text { M. berthae } \\
\text { (ind./ } \mathbf{~ k m}^{2} \text { ) }\end{array}$ & $\begin{array}{c}\text { M. murinus } \\
\text { (ind./ km } \mathbf{~}^{2}\end{array}$ & $\begin{array}{c}\text { M. coquereli } \\
\text { (ind./ } \mathbf{k m}^{2} \text { ) }\end{array}$ & $\begin{array}{c}\text { C. medius } \\
\text { (ind./ } \mathbf{k m}^{2} \text { ) }\end{array}$ & $\begin{array}{c}\text { P. pallescens } \\
\text { (ind./ } \mathbf{k m}^{2} \text { ) }\end{array}$ \\
\hline \multirow{2}{*}{ Ambadira } & DS & 94.55 & 34.70 & 115.99 & - & 30.36 \\
& RS & 180.51 & 34.70 & 136.71 & 89.99 & 9.11 \\
\hline \multirow{2}{*}{ Kirindy } & DS & 54.76 & 215.47 & 16.88 & - & 34.43 \\
& RS & 57.31 & 268.12 & 15.26 & 179.84 & 28.33 \\
\hline \multirow{2}{*}{ Corridor } & DS & 68.77 & 289.13 & 22.09 & - & 33.39 \\
& RS & 34.38 & 242.87 & 22.09 & 258.44 & 54.64 \\
\hline \multirow{2}{*}{ RSA } & DS & 25.79 & 563.81 & 33.14 & - & 31.87 \\
& RS & 0.00 & 294.91 & 16.57 & 138.45 & 27.32 \\
\hline
\end{tabular}

In contrast to global (table 7) and stratum level (table 18 in appendix) density estimates calculated reliably by DISTANCE, density estimates by region (as given in table 8 ) represent unweighted means of stratum level estimates. As multiple covariate distance sampling does not allow for reliable calculation of densities per transect, further analyses were based on encounter rates rather than on population densities anyway. 


\section{Habitat classification}

Our ground assessment of the forest only allowed for reliable classification into nondegraded and degraded habitat. This ground-based classification was broadly congruent with a forest classification based on a Landsat ETM 7 picture by Zinner \& Torkler (2005). Forest parts categorized as non-degraded corresponded to forest classes 1 and 2 and our degraded habitat transects are located in forest classes 3 to 6 (figure 3).

Degraded and non-degraded habitat types differed in canopy cover (figure 25a) and canopy height (figure 25b). Canopy cover in non-degraded habitat exceeded that of degraded habitat when considering all transects sampled $\left(\mathrm{MWU}_{7,5}=0.0, \mathrm{p}=0.004\right)$. In order to avoid seasonal effects, we separately tested forest cover from early and late dry season samples, and found corresponding differences in those subsamples (late dry season: $\mathrm{MWU}_{3,4}=0.0$, $\mathrm{p}=0.034$ / early dry season: $\mathrm{MWU}_{4,2}=0.0, \mathrm{p}=0.06$ ). Canopy height was only estimated in late dry season 2007 and found higher in the non-degraded habitat sample $\left(\mathrm{MWU}_{3,4}=0.0\right.$, $p=0.034)$.
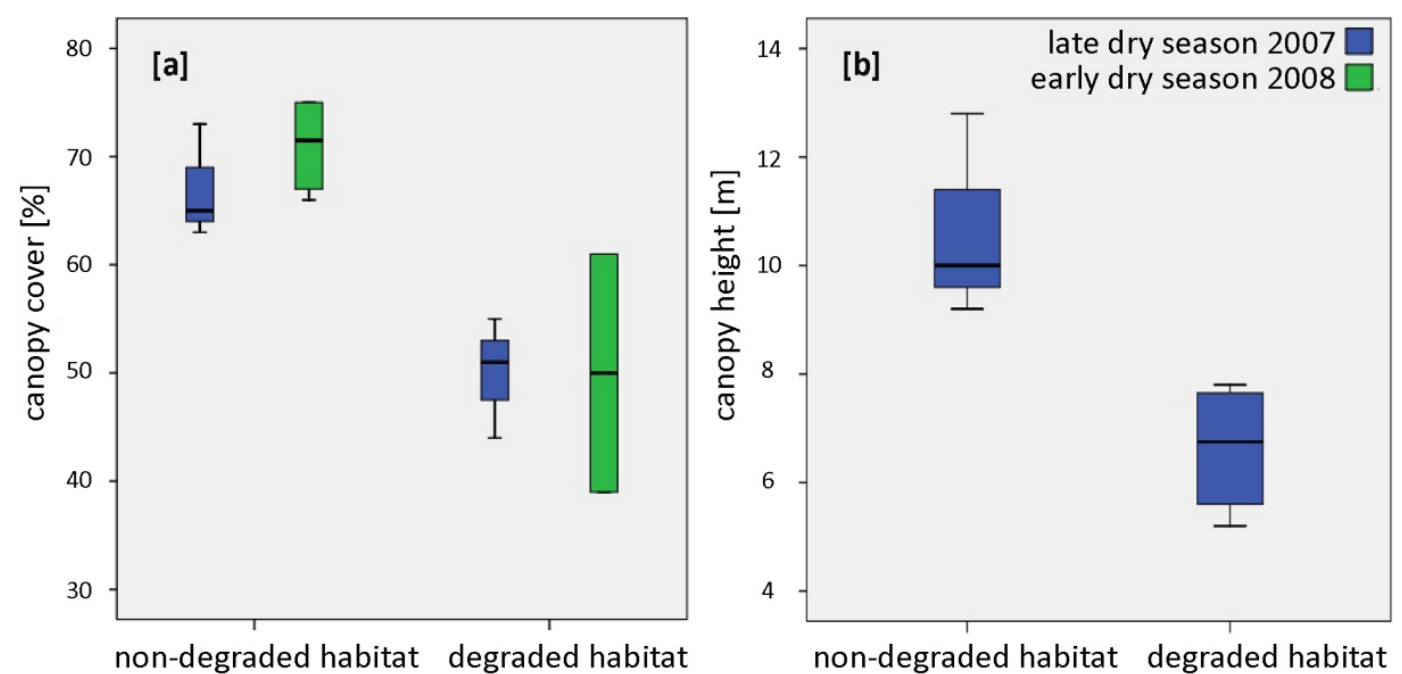

Fig. 25: [a] Canopy cover and [b] canopy height in degraded and non-degraded habitat; transect specific values represent means of 21 sampling plots respectively

Surprisingly this difference was neither associated with larger DBH, i.e. with the size of mature trees $\left(\mathrm{DBH} \geq 10 \mathrm{~cm}: \mathrm{MWU}_{7,5}=9.0, \mathrm{p}=0.167\right)$, and only a non-significant trend indicated that trees older than ten years were larger in non-degraded habitat $(5 \mathrm{~cm} \leq \mathrm{DBH}<10 \mathrm{~cm}$ : $\mathrm{MWU}_{7,5}=7.0, \mathrm{p}=0.088$; figure 26). Higher canopy in non-degraded habitats despite a lack of difference in the $\mathrm{DBH}$ of mature trees might be caused by particularly tall tree species that occur only in non-degraded habitats, but we cannot clarify this question, as we did not identify tree species. 


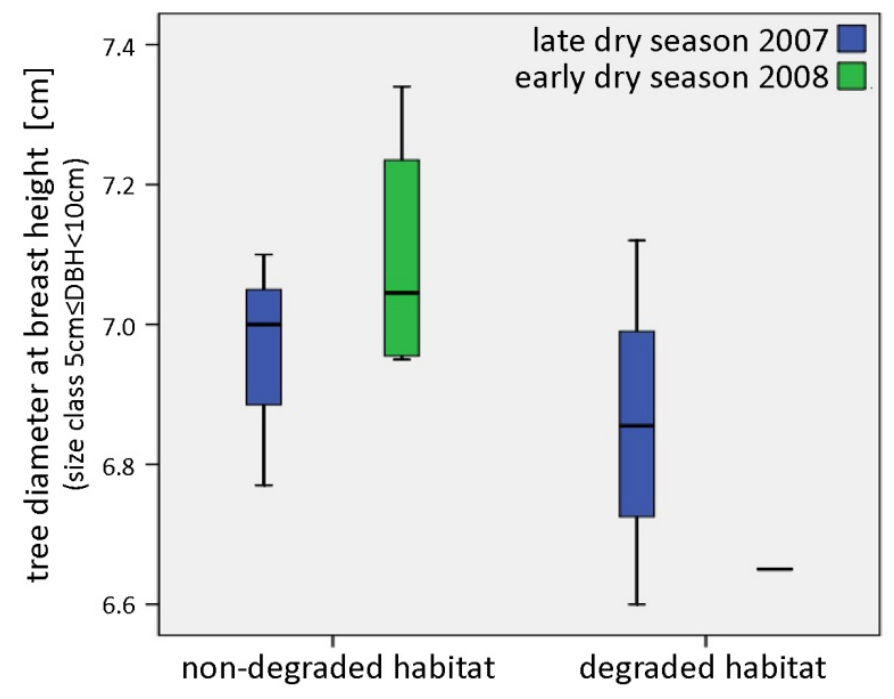

Fig. 26: $\mathrm{DBH}$ of trees of size class $5 \mathrm{~cm} \leq \mathrm{DBH}<10 \mathrm{~cm}$ in degraded and nondegraded habitat; transect specific mean values are based on means of four trees per each of 21 plots, adding up to 84 trees per transect

Degraded and non-degraded habitat did not differ in tree density of any size class $\left(\mathrm{DBH} \geq 10 \mathrm{~cm}: \mathrm{MWU}_{7,5}=8.0, \mathrm{p}=0.123 ; 5 \mathrm{~cm} \leq \mathrm{DBH}<10 \mathrm{~cm}: \mathrm{MWU}_{7,5}=11.0, \mathrm{p}=0.167\right)$. Finally, we could not detect differences in counts of regenerating trees $\left(1 \mathrm{~cm} \leq \mathrm{DBH}<5 \mathrm{~cm}: \mathrm{MWU}_{3,4}=4.0\right.$, $\mathrm{p}=0.480$ ) or understory density (late dry season: $\mathrm{MWU}_{3,4}=2.0, \mathrm{p}=0.150$ / early dry season: $\left.\mathrm{MWU}_{3,3}=2.0, \mathrm{p}=0.355\right)$. However, canopy cover was negatively related to the mean distance of trees from the center point in both size categories (trees older than ten years with $5 \mathrm{~cm} \leq \mathrm{DBH}<10 \mathrm{~cm}$ : Spearman $\mathrm{r}=-0.637, \mathrm{n}=12, \mathrm{p}=0.026$; mature trees with $\mathrm{DBH} \geq 10 \mathrm{~cm}$ : $r=-$ $0.637, n=12, p=0.026$; figure 27).

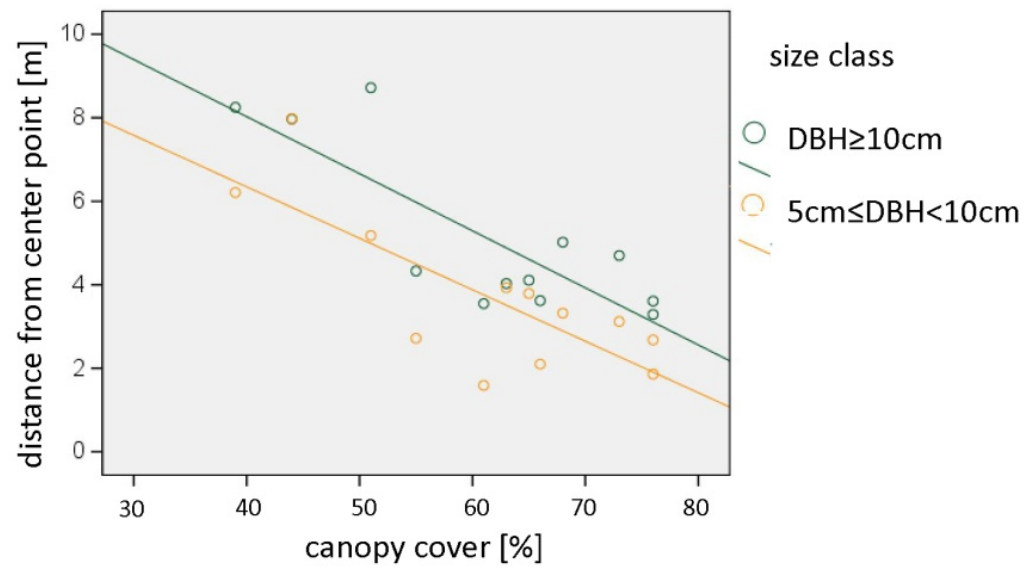

Fig. 27: Correlation mean distances of trees from center point with canopy cover; size class $\mathrm{DBH} \geq 10 \mathrm{~cm}$ (green); size class $5 \mathrm{~cm} \leq \mathrm{DBH}<10 \mathrm{~cm}$ (orange)

Moreover, mean distances from PCQM center points in trees of the two size classes were positively correlated (Spearman $r=0.846, n=12, p=0.001$; figure 28). 


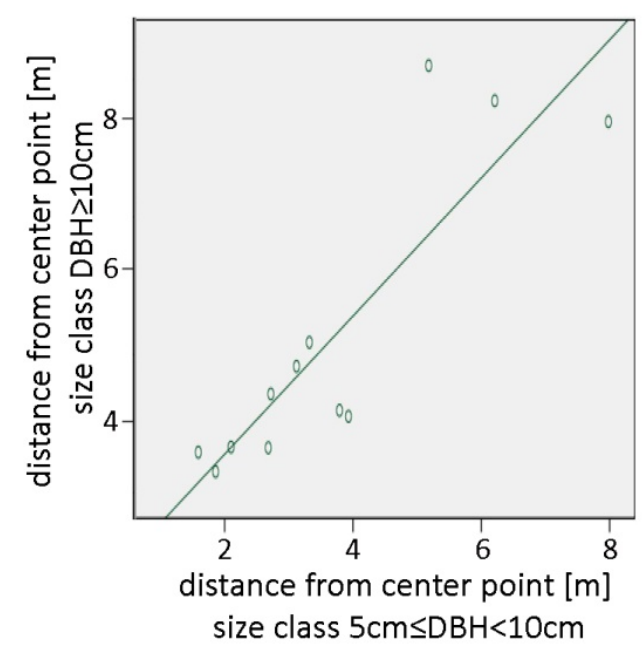

Fig. 28: Correlation of mean distances from center point of trees of size class $\mathrm{DBH} \geq 10 \mathrm{~cm}$ with those of size class $5 \mathrm{~cm} \leq \mathrm{DBH}<10 \mathrm{~cm}$

Thus, there are indirect indications that closed canopy cover in non-degraded habitat is associated with a higher density of mature trees $(\mathrm{DBH} \geq 10 \mathrm{~cm})$ as well as of trees with $5 \mathrm{~cm} \leq \mathrm{DBH}<10 \mathrm{~cm}$.

\section{Methodological tests of survey design}

To validate our survey design, we tested the distribution of transect categories among forest regions, as well as in relation to habitat characteristics. Transect categories were not equally distributed across forest regions, since transects of type "path" were sampled in Kirindy forest only. However, even distribution of transect categories among forest regions was not an assumption to be met and transect type did not affect encounter rates. Mean distances from the nearest village were not significantly different between transect classes (ANOVA $\mathrm{F}=0.889, \mathrm{df}_{1}=2, \mathrm{df}_{2}=33, \mathrm{p}=0.421$ ). Moreover, the number of transects located in degraded and non-degraded habitat did not differ in any transect category $\left(\chi^{2}=1.110, d f=2, p=0.574\right)$. Therefore, our survey design qualified well to include anthropogenic impacts by those proxies in the analysis of regional lemur distribution patterns.

\section{Determinants of regional mouse lemur distribution: data exploration}

\section{Cheirogaleid distribution across forest regions}

On a regional level, $M$. berthae population densities apparently decreased from north to south (highest in Ambadira Forest, lowest in RSA). However, regional differences in $M$. berthae encounter rates were not significant neither during the dry (Kruskal-Wallis $\mathrm{H}(3$, $n=34)=4.080, p=0.253)$, nor in the rainy season $(H(3, n=25)=4.246, p=0.236$; figure 29). 


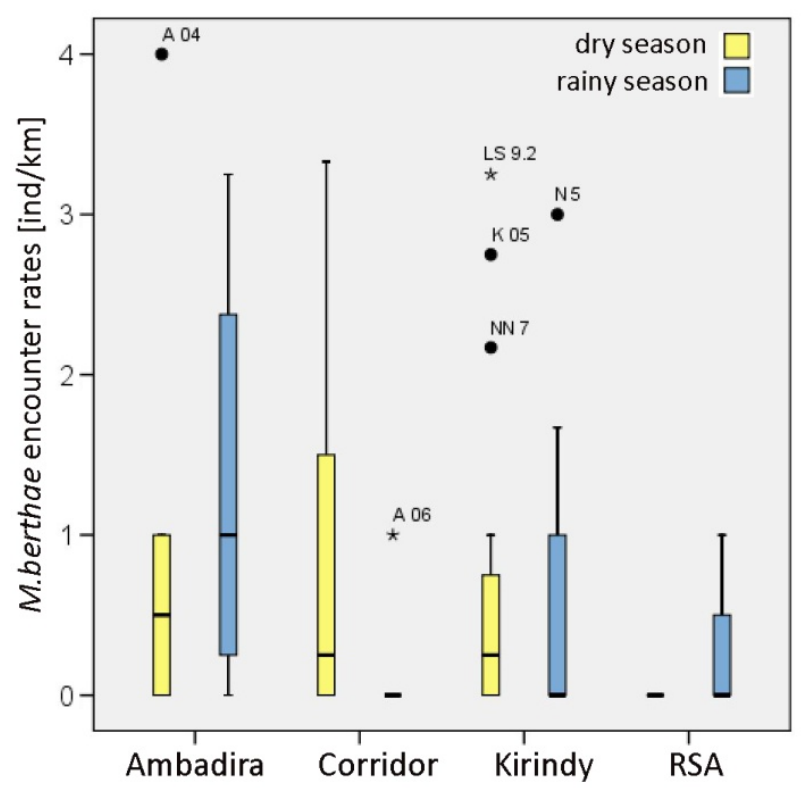

Fig. 29: Microcebus berthae encounter rates per forest region in dry (yellow) and rainy season (blue)

Microcebus murinus' regional distribution was diametrically opposed to that of $M$. berthae (population densities highest in RSA and lowest in Ambadira). Based on pooled dry season data, encounter rates differed between forest regions $(H(3, n=34)=11.392, p=0.01)$, being highest in RSA, followed by the corridor and Kirindy, and lowest in Ambadira region (figure 30). However, no differences between regions emerged when considering pooled rainy season encounters $(H(3, n=25)=4.510, p=0.211)$.

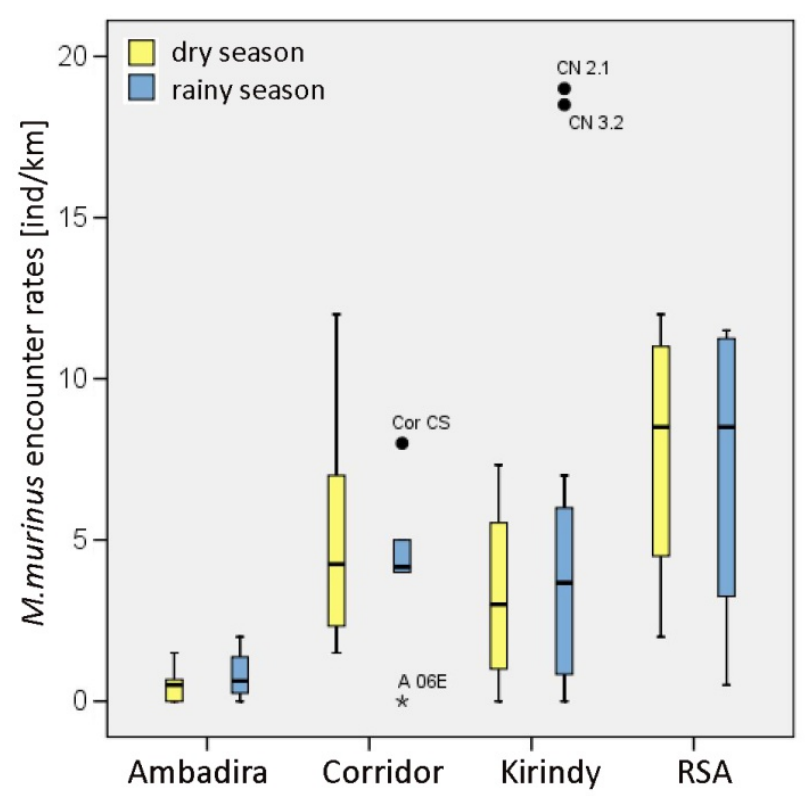

Fig. 30: Microcebus murinus encounter rates per forest region in dry (yellow) and rainy season (blue)

Encounter rates in $M$. coquereli significantly differed between forest regions during dry $(\mathrm{H}(3$, $n=34)=8.120, p=0.044$ ) as well as during rainy season surveys (Kruskal-Wallis $H(3$, 
$n=25)=10.157, p=0.017$ ). In our dry season surveys, encounter rates were highest in Ambadira and RSA, followed by the corridor and Kirindy. Rainy season encounter rates in Ambadira were slightly higher than those of the dry season, but lower in RSA, the corridor, and Kirindy (figure 31).

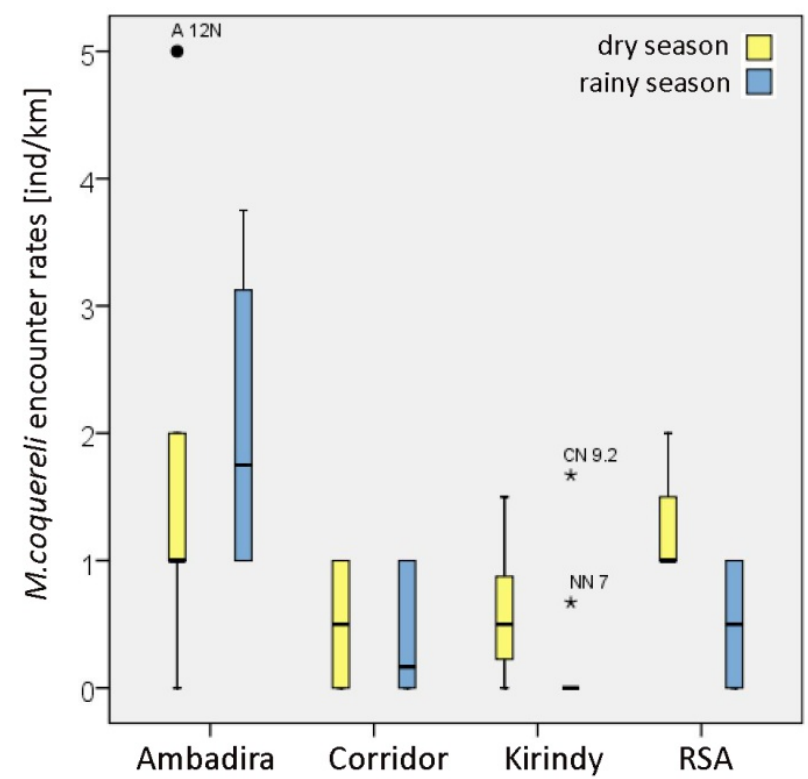

Fig. 31: Mirza coquereli encounter rates per forest region in dry (yellow) and rainy season (blue)

In contrast, no differences in encounter rates between forest regions appeared in either $P$. pallescens $(P$. pallescens, dry season: $H(3, n=34)=0.162, p=0.983$; rainy season: $H(3$, $n=25)=2.605, p=0.457$; figure 32$)$ or $C$. medius $(H(3, n=25)=5.733, p=0.125$; figure 33).

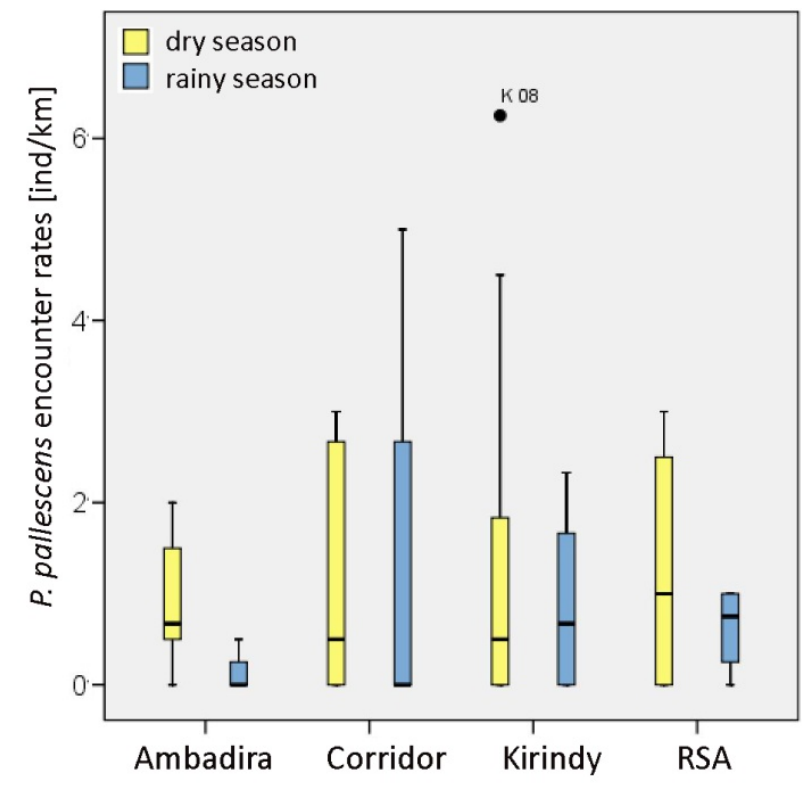

Fig. 32: Phaner pallescens encounter rates per forest region in dry (yellow) and rainy season (blue)

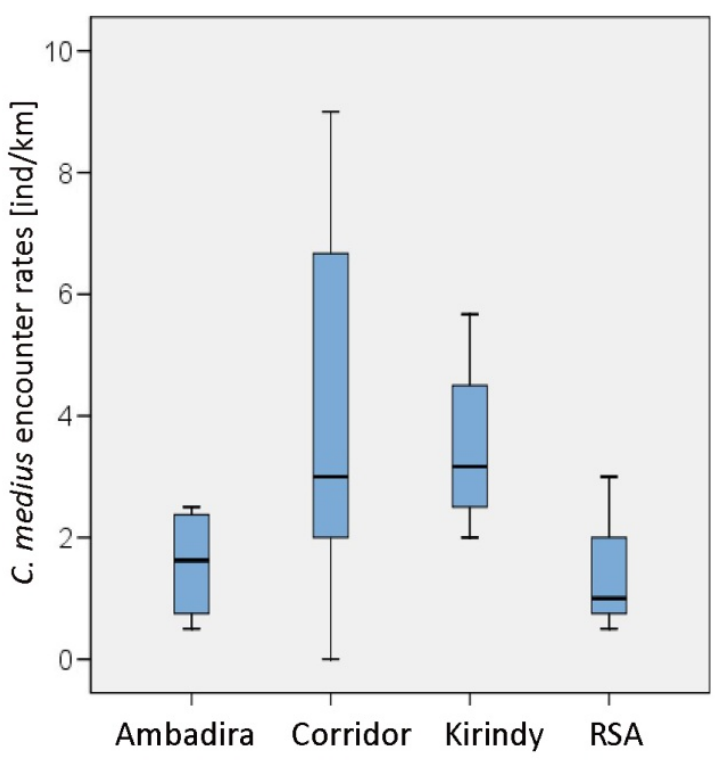

Fig. 33: Cheirogaleus medius encounter rates per forest region in the rainy season (blue) 


\section{Cheirogaleid distribution across heterogeneous habitat}

In the rainy season, we found a non-significant trend in $M$. berthae towards higher encounter rates on non-degraded than on degraded habitat transects $\left(\mathrm{MWU}_{11,14}=-2.199\right.$, $p=0.051)$, whereas dry season encounter rates did not differ between degraded and nondegraded habitat $\left(\mathrm{MWU}_{16,18}=-0.997, \mathrm{p}=0.365\right.$; figure 34).

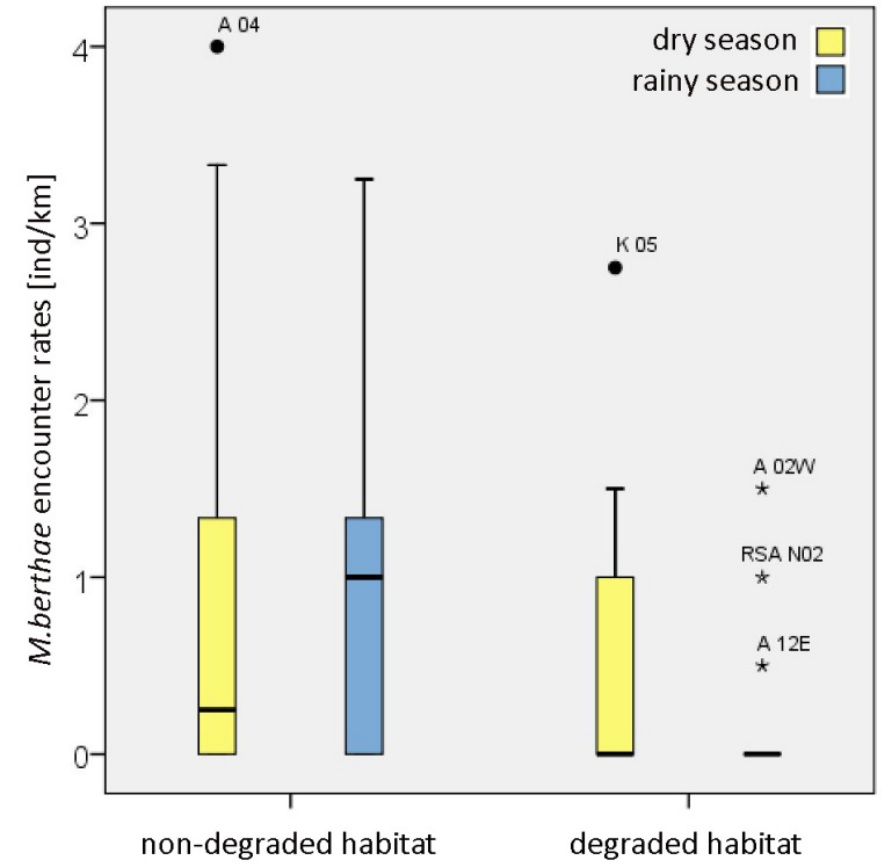

Fig. 34: Microcebus berthae encounter rates in degraded and non-degraded habitat in dry (yellow) and rainy season (blue) respectively

In either season $M$. berthae was present on the majority of transects in non-degraded forest, but only on a small number of transects in degraded habitat (figure 35). However, proportions of transects colonized by $M$. berthae in degraded and non-degraded habitat differed only during the rainy $\left(\chi^{2}=4.573, d f=1, p=0.032\right)$, but not during the dry season $\left(\chi^{2}=1.889, d f=1, p=0.169\right)$.

Regarding all transects surveyed, $M$. berthae's encounter rates seem to seasonally differ (figure 34), with rainy season rates exceeding those of the dry season in non-degraded habitat and vice versa in degraded habitat. Data for pairwise tests of seasonal changes in population densities across heterogeneous habitat were limited to those transects that were surveyed at least once in each season. This small sample did not indicate significant seasonal differences in $M$. berthae's encounter rates regardless of habitat degradation (degraded habitat: Wilcoxon $Z=0.000, n=13, p=1.0$; non-degraded habitat: Wilcoxon $Z=-0.702, n=10$, $\mathrm{p}=0.483$ ). Microcebus berthae also appeared to occur on a lower proportion of degraded habitat transects and on a slightly higher proportion of non-degraded habitat transects 
during the rainy season compared to the dry season (figure 35a, b). However, based on paired analyses of transects sampled in either season, we did not find any seasonal differences in the proportion of transects colonized (sign test, degraded habitat: $n=13$, $p=1.0$; non-degraded habitat: $n=10, p=1.0$ ).
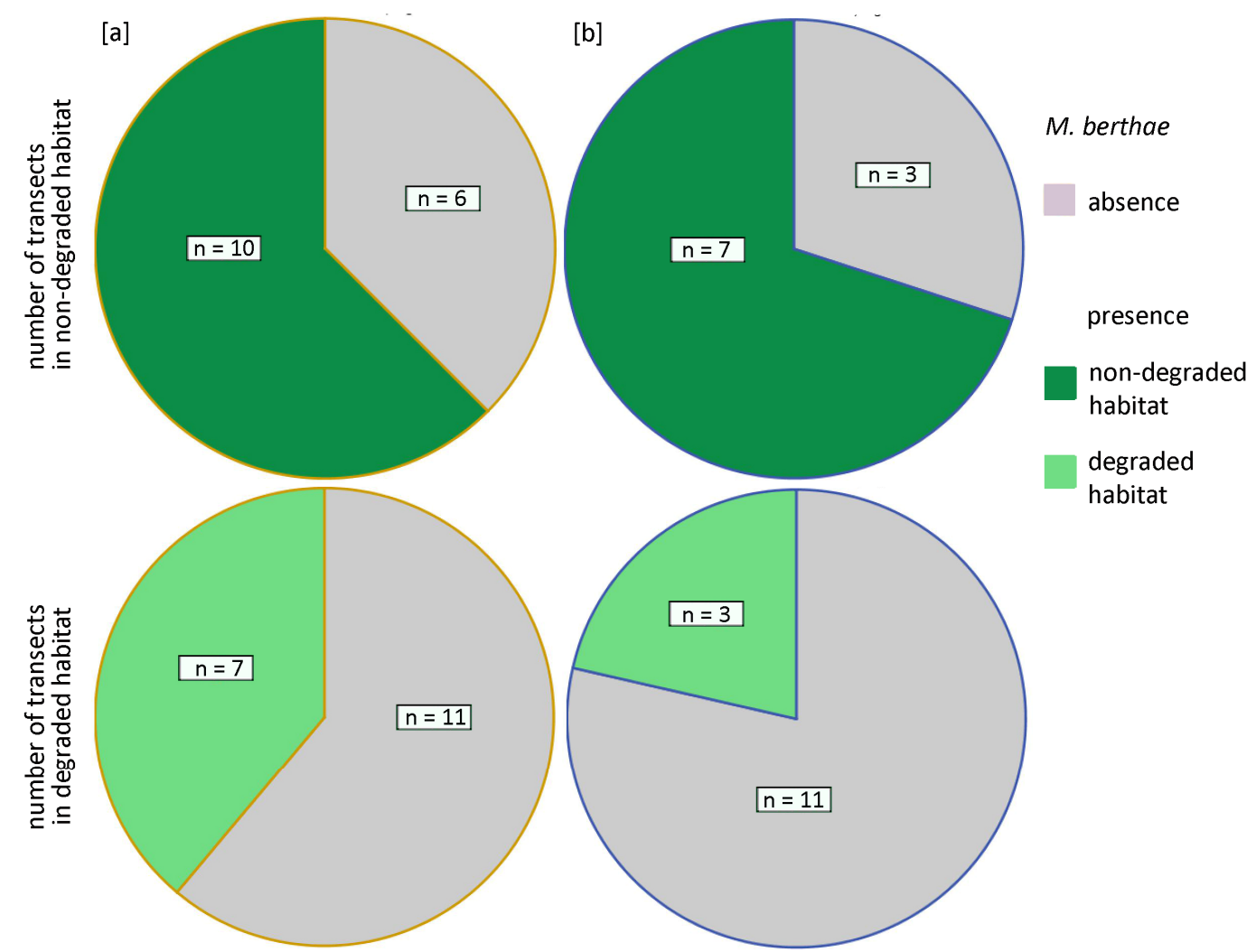

Fig. 35: Number of transects on which $M$. berthae was present/ absent in non-degraded and degraded habitat during [a] dry $(n=34)$ and [b] rainy season $(n=25)$

In contrast, we found a non-significant trend towards higher dry season encounter rates of M. murinus in degraded compared to non-degraded habitat $\left(\mathrm{MWU}_{16,18}=-1.799, \mathrm{p}=0.075\right)$. Lack of significant differences in encounter rates between degraded and non-degraded habitats during the rainy season $\left(\mathrm{MWU}_{11,14}=-1.125, \mathrm{p}=0.267\right)$ was due to two extreme outliers (figure 36), on which we encountered exceptionally high numbers of $M$. murinus individuals even though the habitat was classified as non-degraded. Omitting transects $\mathrm{CN}$ 3.2 and $\mathrm{CN} 2.1$ from analysis, we encountered M. murinus in significantly higher rates in degraded habitat also in the rainy season $\left(\mathrm{MWU}_{9,14}=-2.179, \mathrm{p}=0.029\right)$. 


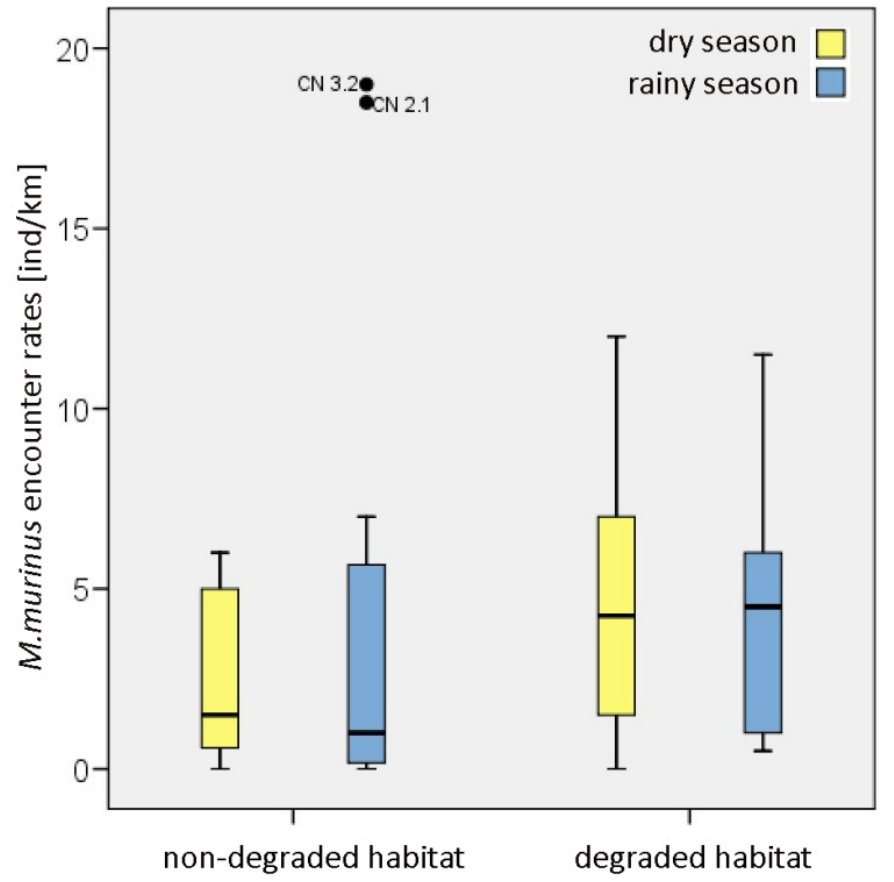

Fig. 36: Microcebus murinus encounter rates in degraded and non-degraded habitat, in dry (yellow; $\mathrm{n}=34$ ) and rainy season (blue; $\mathrm{n}=25$ ) respectively

The number of transects colonized by $M$. murinus in different density classes did not significantly differ between degraded and non-degraded habitat (dry season: $\chi^{2}=4.516, d f=3$, $p=0.211$; rainy season: $\left.\chi^{2}=5.722, d f=3, p=0.126\right)$. Based on the limited sample of transects that were surveyed at least once each season, $M$. murinus encounter rates neither differed between seasons (degraded habitat: Wilcoxon $Z=-1.299, n=13, p=0.194$, non-degraded habitat: Wilcoxon $Z=-0.356, n=10, p=0.722)$.

Likewise, the number of transects populated by $M$. murinus in one of four density classes, did not differ between seasons (degraded habitat: Wilcoxon $Z=0.0, n=13, p=1.0$; non-degraded habitat: Wilcoxon $Z=0.0, n=10, p=1.0)$. However, a striking pattern emerges when regarding the distribution of $M$. murinus density classes across transects of different quality (figure $37 \mathrm{a}, \mathrm{b}$ ). During the dry season, we did never encounter $M$. murinus in high densities in non-degraded habitat, the species rather was absent or represented by low or medium population sizes on the majority of such transects. In degraded habitat, however, we surveyed some more transects with high or medium M. murinus population densities. During the rainy season $M$. murinus colonized most non-degraded habitat transects in low densities and was even absent from some of them. In contrast, $M$. murinus was present on all degraded habitat transects during the rainy season, and occurred on some transects in high, but most frequently in low and medium population densities. Microcebus murinus may 
therefore spread out across heterogeneous habitat during the rainy season to track most pronounced increases in carrying capacity.
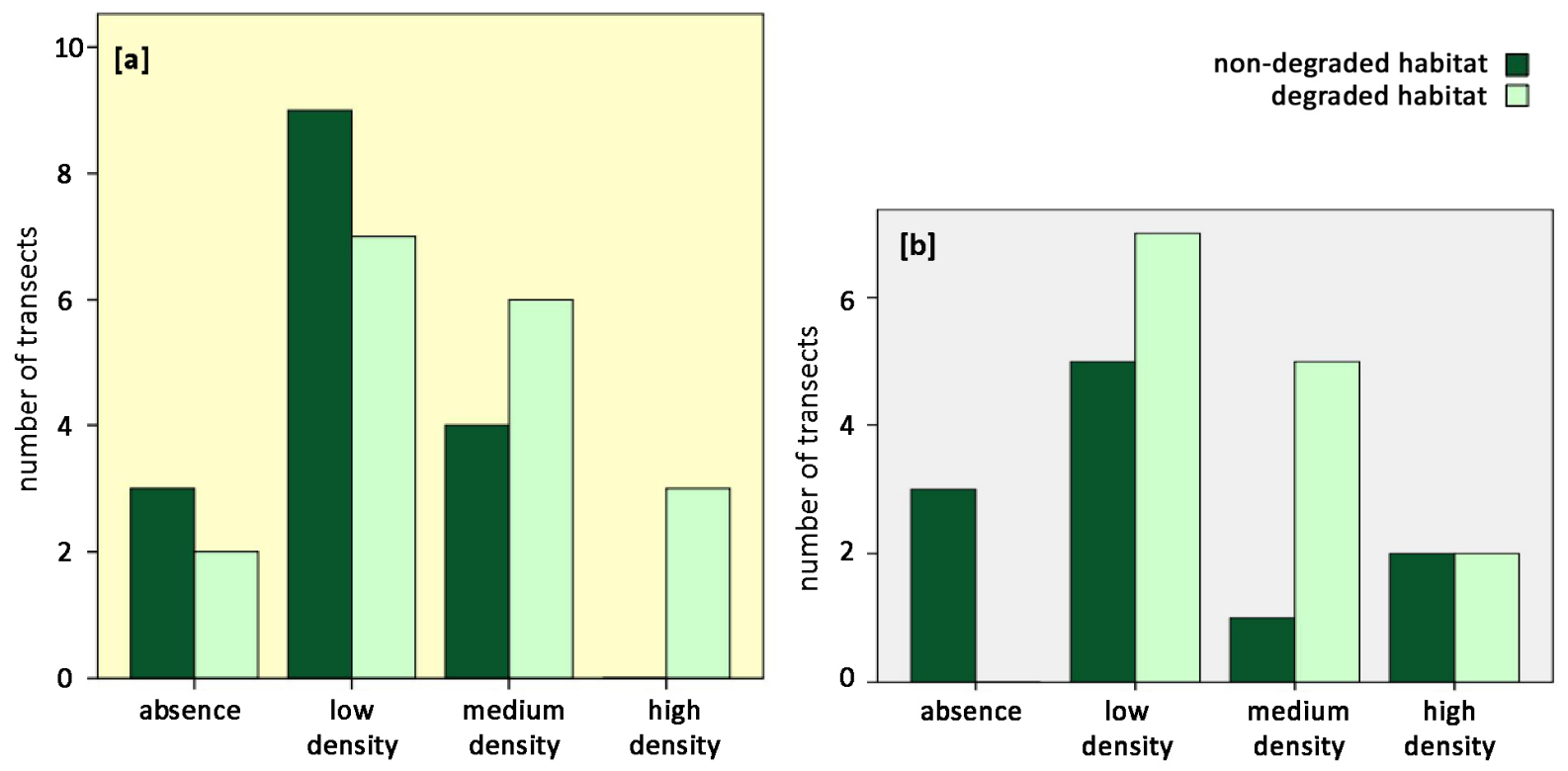

M. murinus density classes

Fig. 37: Number of transects with $M$. murinus in different density classes in non-degraded (dark green) and degraded (light green) habitat during [a] dry $(n=34)$ and [b] rainy season $(n=25)$ respectively

C. medius individuals were encountered more frequently in non-degraded than in degraded habitat during rainy season surveys $\left(\mathrm{MWU}_{11,14}=33.0, \mathrm{p}=0.015\right.$; figure 38$)$. The species was found on almost all transects in degraded as well as in non-degraded habitats, but there were differences in the number of degraded and non-degraded habitat transects harboring different density classes of $C$. medius $\left(\chi^{2}=8.992, d f=3, p=0.029\right.$; figure 39). The species occurred on all non-degraded habitat transects, most frequently in high and medium densities, and only one single transect had few $C$. medius. In contrast, most degraded habitat transects were characterized by low $C$. medius densities, followed by such with medium densities, and we found the species in high densities or absent on one transect respectively. 


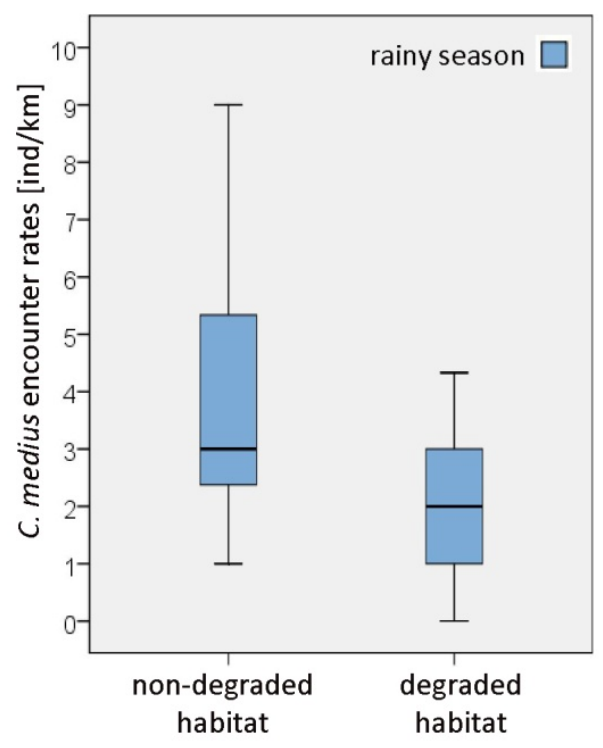

Fig. 38: Cheirogaleus medius rainy season encounter rates in non-degraded $(n=11)$ and degraded $(n=14)$ habitat

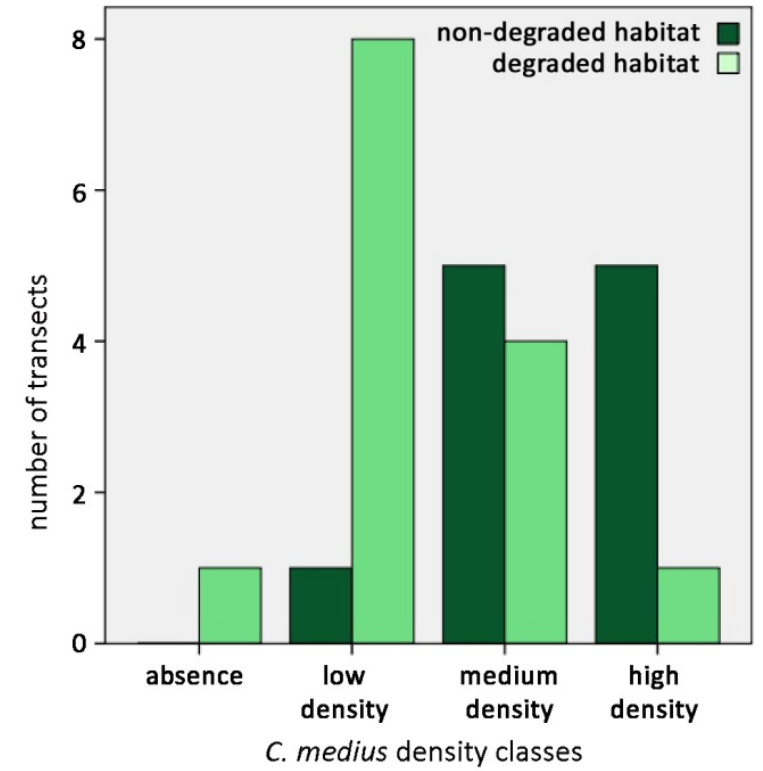

Fig. 39: Number of transects with $C$. medius in different density classes in non-degraded (dark green; $n=11$ ) and degraded (light green; $\mathrm{n}=14$ ) habitat

M. coquereli encounter rates did not differ between degraded and non-degraded habitat neither during dry season surveys ( $M$. coquereli: $\left.\mathrm{MWU}_{16,18}=135.5, \mathrm{p}=0.772\right)$, nor during those of rainy seasons ( $M$. coquereli: $\mathrm{MWU}_{11,14}=72.0, \mathrm{p}=0.809$ ). Despite $M$. coquereli being absent from some more degraded that non-degraded habitat transects during the dry season, the proportion of transects on which the species was present did not differ between degraded and non-degraded habitat ( $d r y$ season: $\chi^{2}=1.209, d f=1, p=0.271$; rainy season: $\chi^{2}=0.017$, $d f=1, p=0.897)$.

Based on only those transects that were sampled in either season, we did not find seasonal differences in $M$. coquereli encounter rates in degraded habitat (Wilcoxon $Z=-$ $1.403, n=13, p=0.161$ ), and only a non-significant trend indicating more dry than rainy season encounters in non-degraded habitat (Wilcoxon $Z=-1.757, n=10, p=0.079$ ). Accordingly, we sampled non-significantly more transects with $M$. coquereli present during the dry than during the rainy season, regardless of habitat degradation (sign test, degraded habitat: $p=0.070, n=13$; non-degraded habitat: $p=0.063, n=10$; figure 40 ). This pattern is most likely a result of improved detection probabilities during the dry season due to reduced foliage or behavioral changes (cf. Lehman 2006b). 


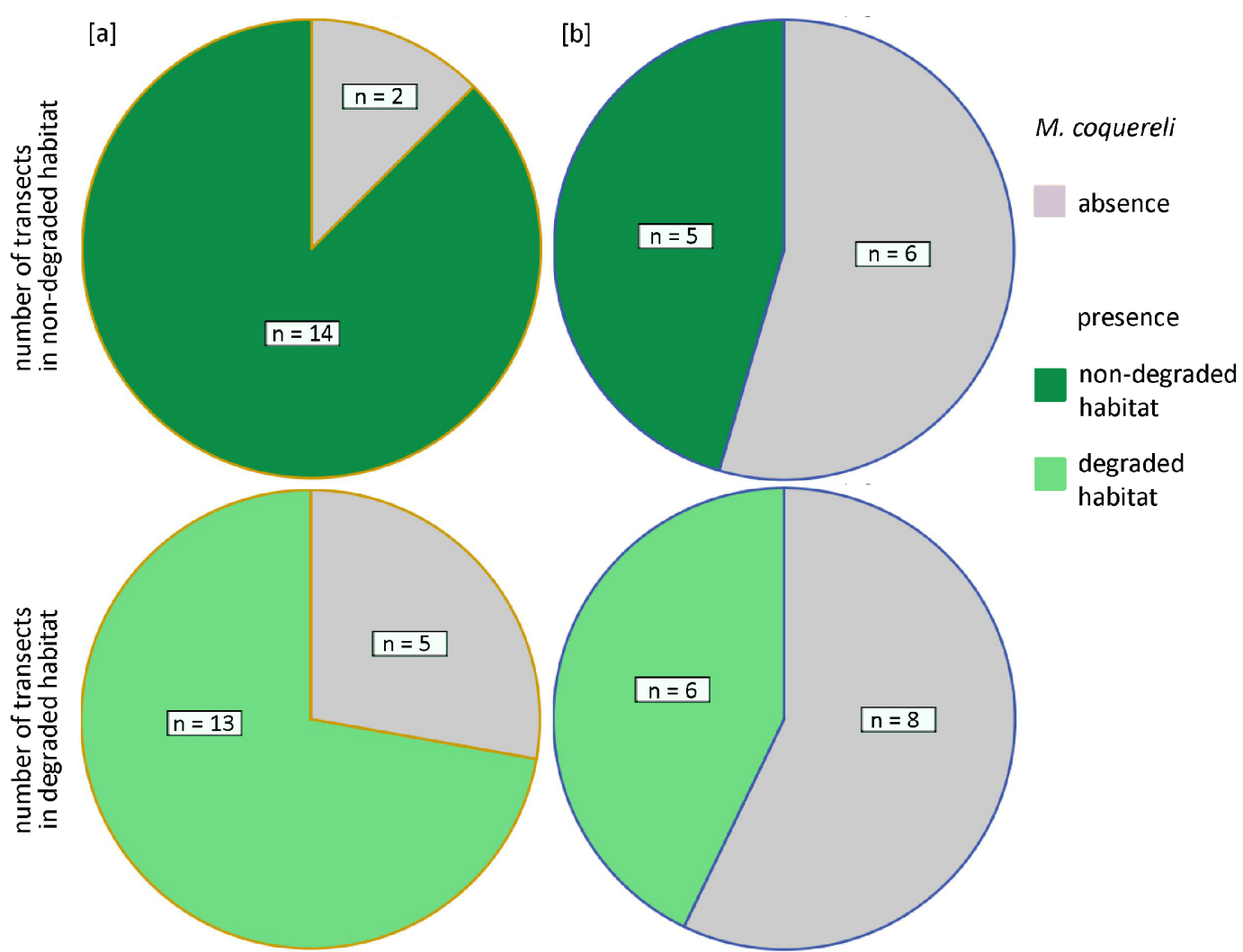

Fig. 40: Number of transects on which $M$. coquereli was present/ absent in non-degraded and degraded habitat during [a] dry $(n=34)$ and [b] rainy season $(n=25)$

Finally, we did not find differences in encounter rates of $P$. pallescens between degraded and non-degraded habitat in either season (dry season: $\mathrm{MWU}_{16,18}=135.5, \mathrm{p}=0.772$; rainy season: $\left.\mathrm{MWU}_{11,14}=63.0, \mathrm{p}=0.467\right)$.

\section{Sex-specific cheirogaleid captures in degraded and non-degraded habitat}

Captures from line trapping roughly reconfirmed these differences in cheirogaleid population densities between degraded and non-degraded habitat. We captured more $M$. berthae males in non-degraded than in degraded habitat (all seasons: $\mathrm{MWU}_{32,20}=232.0$, $p=0.041$; dry season: $\left.M \mathrm{MU}_{15,9}=39.0, \mathrm{p}=0.065\right)$, but no differences emerged in female captures (all seasons: $\mathrm{MWU}_{32,20}=276.0, \mathrm{p}=0.319$; dry season: $\mathrm{MWU}_{15,9}=61.0, \mathrm{p}=0.685$; figure $41 a, b)$. 

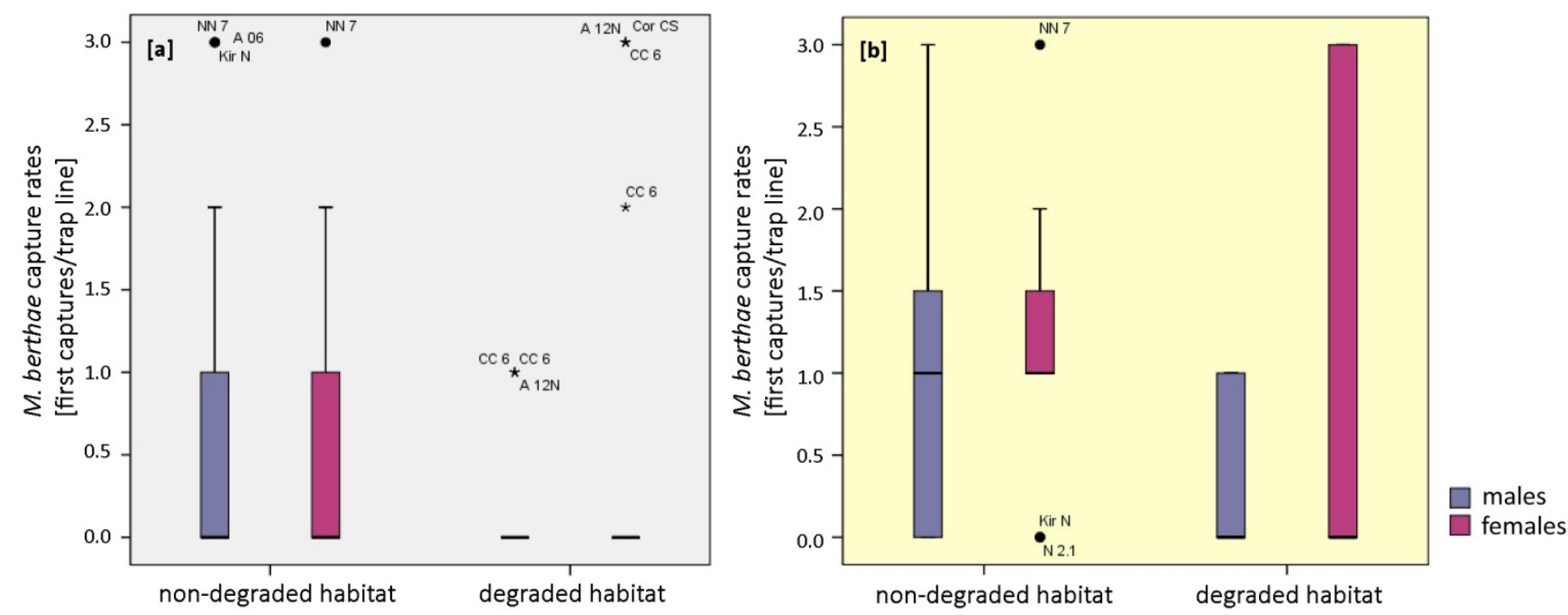

Fig. 41: Microcebus berthae male and female capture rates in degraded and non-degraded habitat; [a] all seasons and [b] in dry season

More M. murinus individuals of both sexes were captured in degraded habitat if analyzing tapping data of all seasons (males: $\mathrm{MWU}_{32,20}=219.5, \mathrm{p}=0.041$; females: $\mathrm{MWU}_{32,20}=219.5$, $p=0.033$; figure 42). Based only on dry season data, differences in first captures between habitats of different quality turned to non-significance (males: $\mathrm{MWU}_{15,9}=41.0, \mathrm{p}=0.101$; females: $\mathrm{MWU}_{15,9}=38.5, \mathrm{p}=0.084$; figure 43a/ rainy season captures are depicted in $43 \mathrm{~b}$ for comparison).

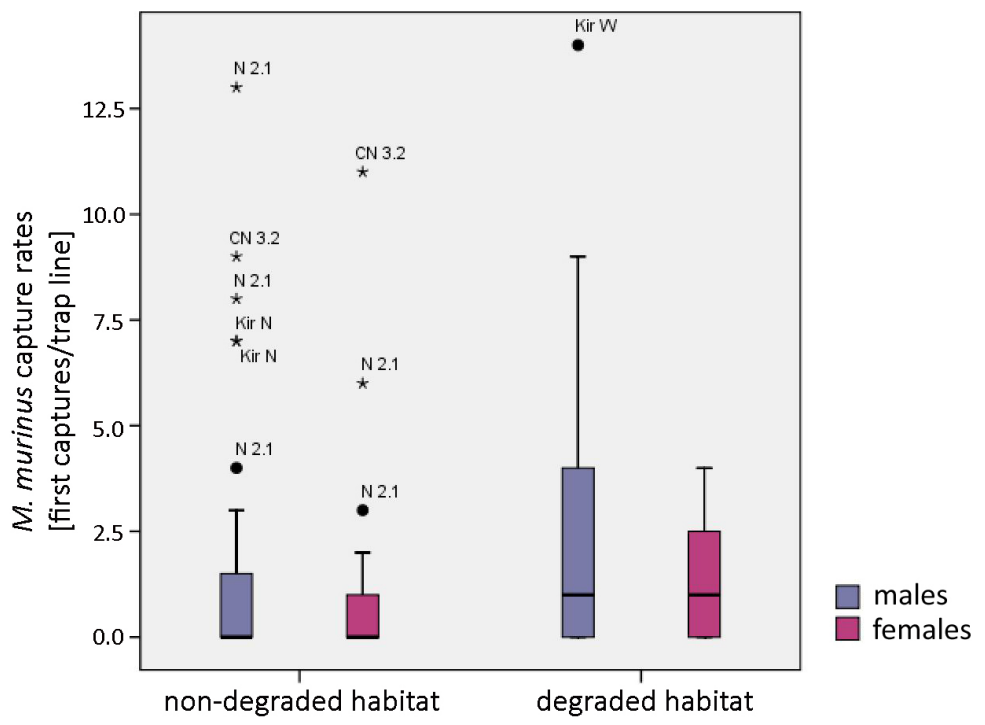

Fig. 42: Microcebus murinus male and female capture rates in degraded and non-degraded habitat pooled over seasons

Capture rates in $M$. coquereli did not differ between habitats of different quality, neither in males (all seasons: $\mathrm{MWU}_{32,20}=311.0, \mathrm{p}=0.760$; dry season: $\mathrm{MWU}_{15,9}=61.0, \mathrm{p}=0.550$ ), nor in females (all seasons: $\mathrm{MWU}_{32,20}=315.5, \mathrm{p}=0.834$; dry season: $\mathrm{MWU}_{15,9}=65.5, \mathrm{p}=0.907$ ). Neither did C. medius captures reconfirm higher population densities in non-degraded 
habitat, as indicated by distance samples (males, rainy season: $\mathrm{MWU}_{17,11}=79.5, \mathrm{p}=0.427$; females rainy season: $\left.\mathrm{MWU}_{17,11}=76.0, \mathrm{p}=0.336\right)$.

\section{Sex-ratio in M. murinus captures in degraded and non-degraded habitat}

Microcebus murinus dry season captures varied significantly with the factor sex, in that we captured more males than females in both, degraded and non-degraded habitat (2-factorial ANOVA $\left.\mathrm{F}=6.703, \mathrm{df}_{1}=35, \mathrm{df}_{2}=12, \mathrm{p}=013\right)$. Moreover, we captured non-significantly more individuals in degraded than in non-degraded habitat (2-factorial ANOVA $F=3.129, \mathrm{df}_{1}=39$, $\mathrm{df}_{2}=16, \mathrm{p}=0.084$ ). However, there were no interactions of sex and habitat degradation (2factorial ANOVA $F=1.087, d f=1, p=0.303$ ). Therefore, our data did not confirm more females to be active in secondary forest during the dry season than in primary forest (Ganzhorn \& Schmid 1998; figure 43a, b).
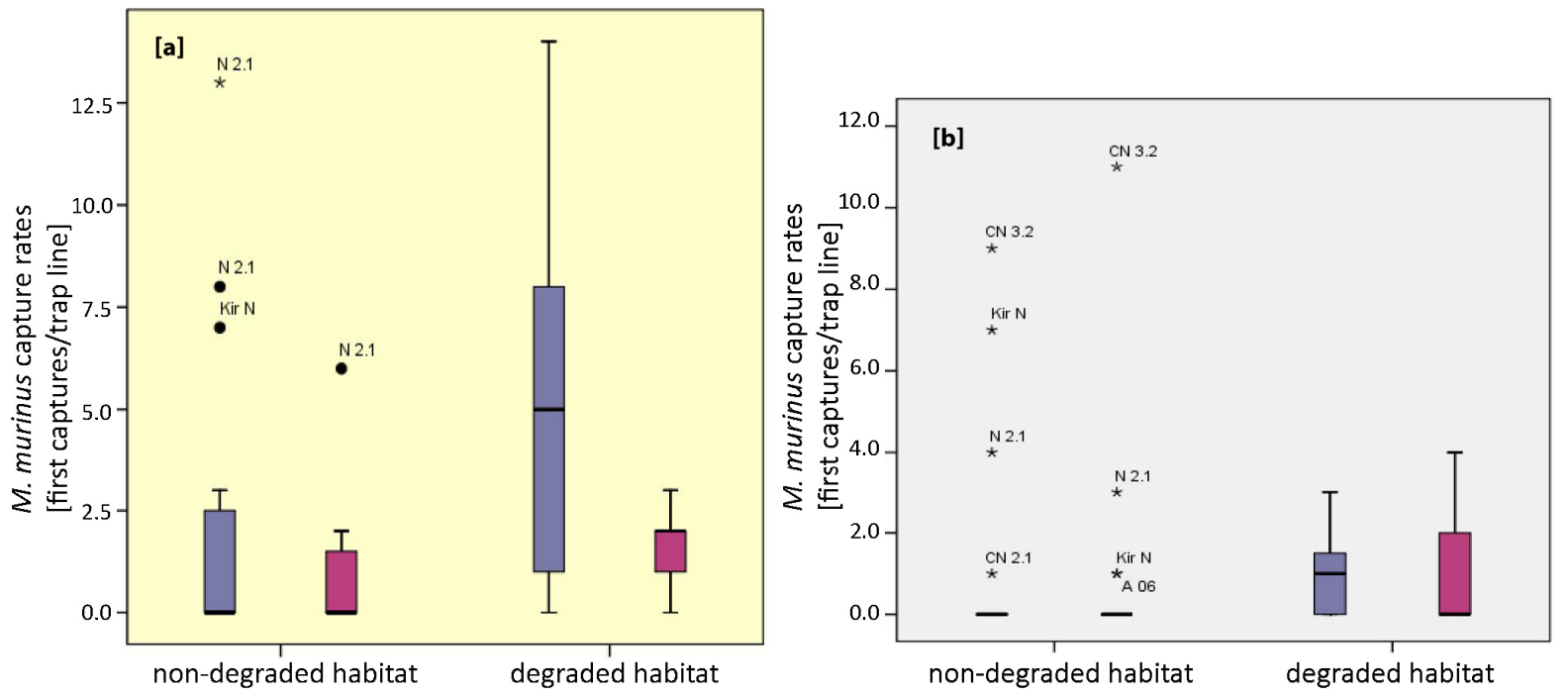

Fig. 43: Microcebus murinus male and female capture rates in degraded and non-degraded habitat in [a] dry season and $[b]$ rainy season

\section{Cheirogaleid distribution in relation to distance from the closest village}

We encountered significantly more $M$. berthae individuals with increasing distance from the closest village during the dry season (Spearman $r=0.529, n=34, p=0.001$ ), but only a nonsignificant trend appeared during the rainy season $(r=0.268, n=25, p=0.098)$. Microcebus murinus encounter rates were reverse proportionate to the distance from the closest village only during the dry season $(r=-0.442, n=34, p=0.004$; rainy season: $r=-0.074, n=25, p=0.363$; figure $44 a, b)$. 

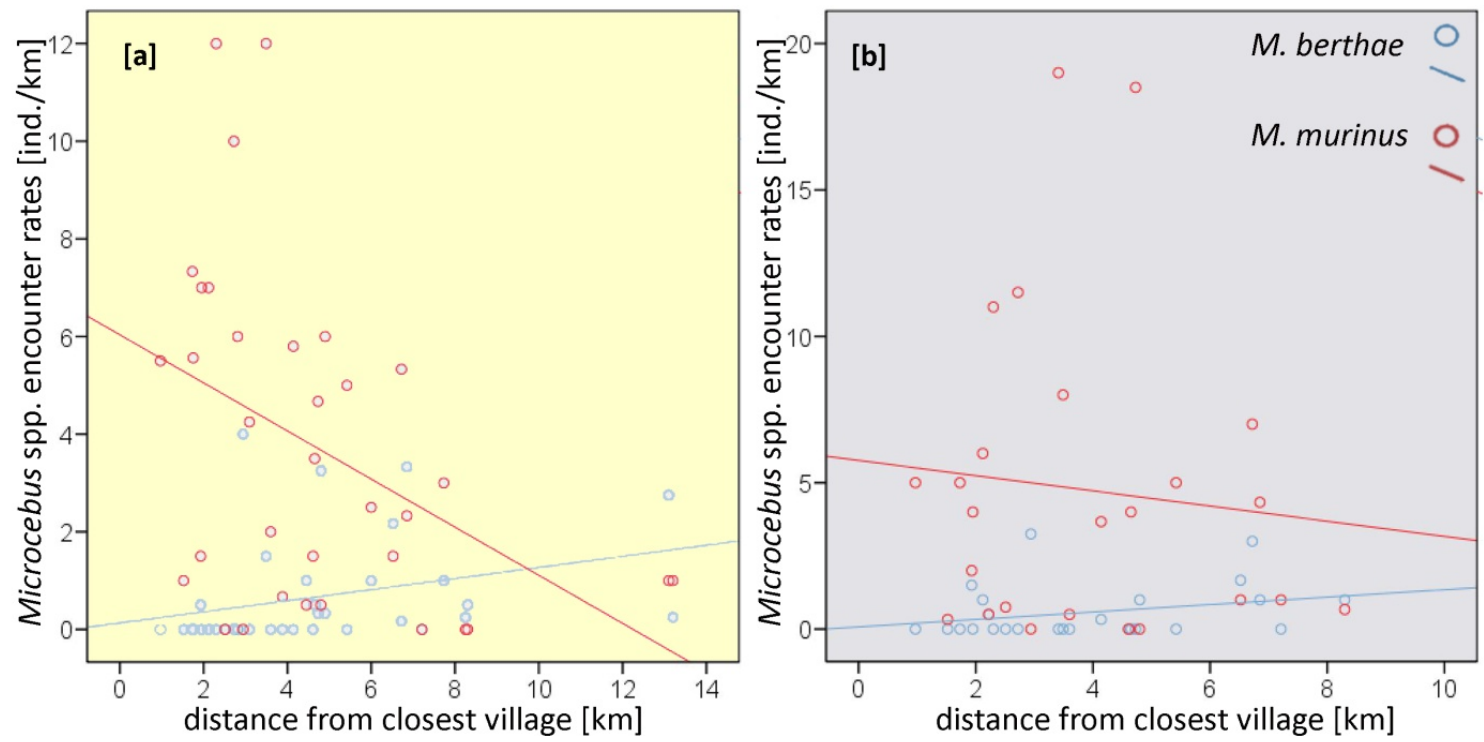

Fig. 44: Microcebus spp. encounter rates vs. distance from closest village in [a] dry and [b] rainy season

Similar to $M$. berthae, $C$. medius $(r=0.391, n=25, p=0.027)$ and $P$. pallescens (dry season: $r=0.572, n=34, p=0.000$; rainy season: $r=0.384, n=25, p=0.029$ ) seem to evade the proximity of villages. Mirza coquereli's distribution across transects, however, was not related to the distance from villages (dry season: $r=-0.052, n=34, p=0.385$; rainy season: $r=0.053, n=25$, $p=0.400$; figure $45 a, b)$.
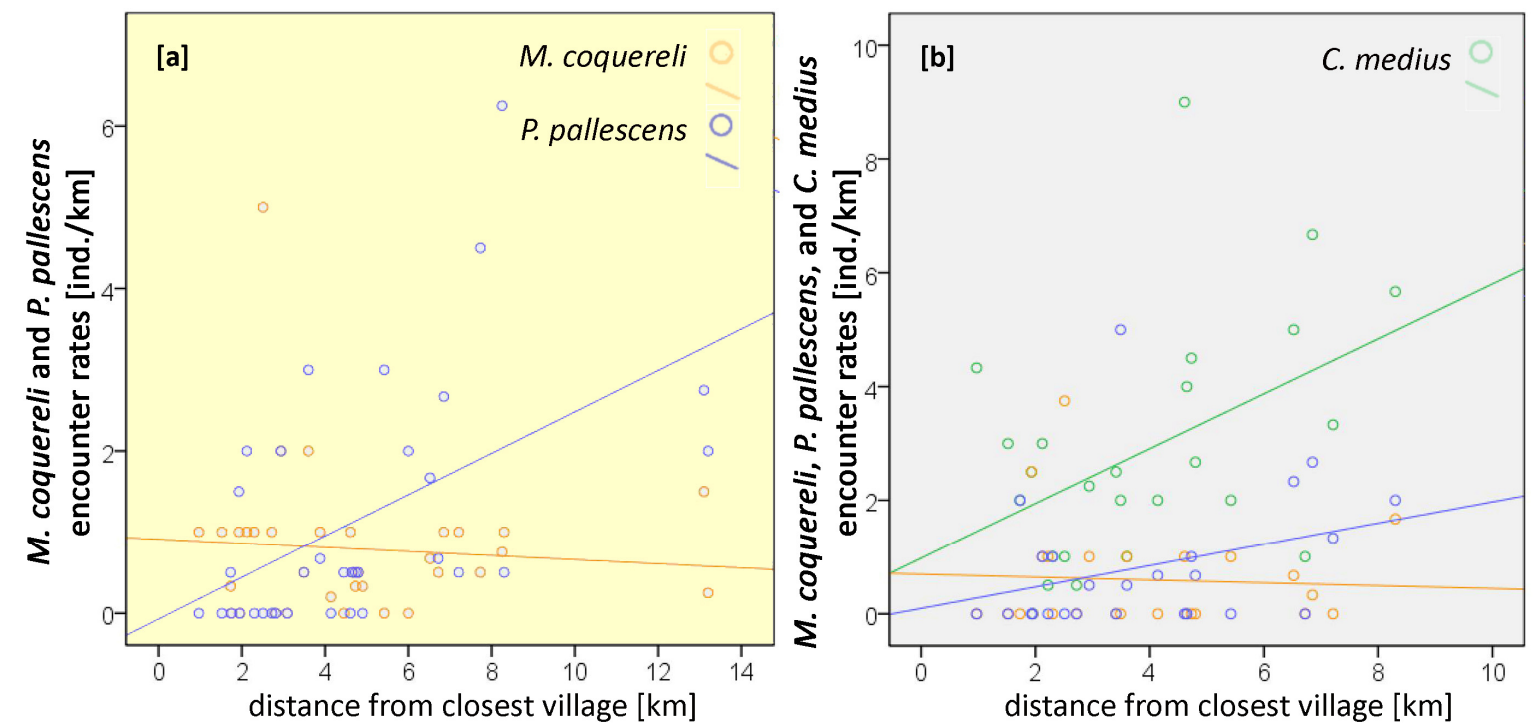

Fig. 45: Greater cheirogaleids' encounter rates in relation to the distance from the closest village in [a] dry and [b] rainy season

\section{Interactions of interspecific interactions and environmental factors}

$M$. berthae encounter rates differed between transects classified by $M$. murinus' encounters only during the dry season in degraded habitat (Kruskal-Wallis $H(3, n=18)=9.419, p=0.024)$. We did not encounter any $M$. berthae individuals in degraded habitat when $M$. murinus was absent or occurred at medium densities. On transects with low densities of the congener, $M$. 
berthae's encounter rates were highest, but the species occurred also on transect with high M. murinus encounter rates (figure $46 a, b$ ). No significant differences in encounter rates appeared in non-degraded habitat during the dry season $(H(2, n=16)=2.946, p=0.229)$ or, regardless of habitat type, during the rainy season (non-degraded: $H(3, n=11)=4.139$, $p=0.247$; degraded: $H(2, n=14)=0.697, p=0.706)$. However, figure $46 b$ makes obvious that during the rainy season $M$. berthae only occurs on transects where $M$. murinus is absent or occurs in low densities.
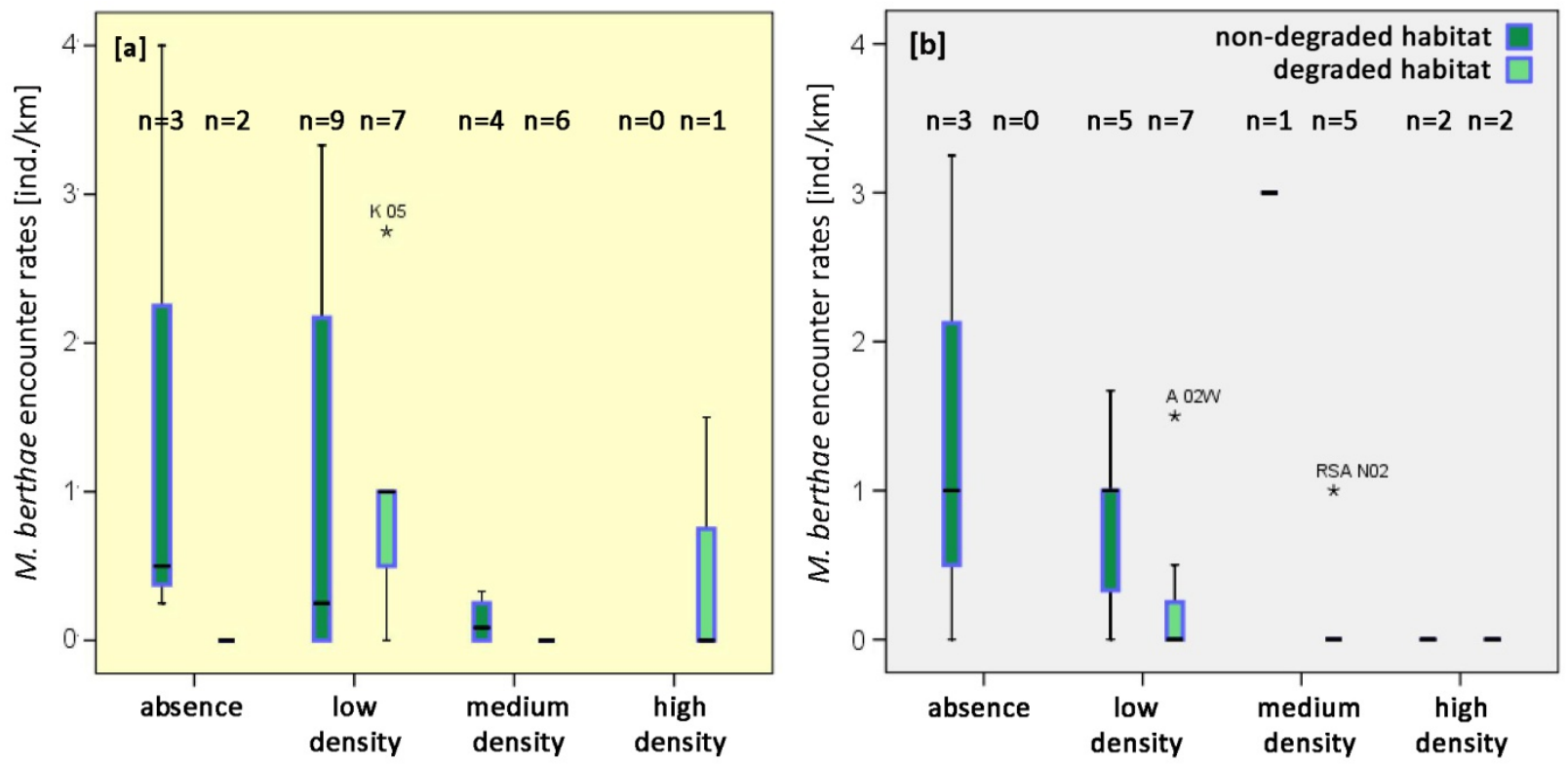

M. murinus density classes

Fig. 46: Microcebus berthae [a] dry and [b] rainy season encounter rates on transects with varying density classes of $M$. murinus, in non-degraded (dark green) and degraded (light green) habitat respectively

We did not find differences in $M$. murinus' encounter rates between transects with $M$. berthae either present or absent, regardless of season or habitat degradation (dry season, non-degraded: $\mathrm{MWU}_{6,10}=21.0, \mathrm{p}=0.327$; dry season, degraded: $\mathrm{MWU}_{11,7}=25.5, \mathrm{p}=0.238$; rainy season, non-degraded: $\mathrm{MWU}_{4,7}=11.0, \mathrm{p}=0.567$; rainy season, degraded: $\mathrm{MWU}_{11,3}=11.5$, $\mathrm{p}=0.456)$.

\section{Determinants of regional mouse lemur distribution: Log-linear models}

The distribution of $M$. berthae was influenced in both seasons by habitat degradation and $M$. coquereli, and during the dry season additionally by distance to the closest village. In the dry season, encounter rates of $M$. berthae (ER_Mb.ds) significantly rose with increasing distance from the closest village (dist.village: $B=0.211, d f=1, p=0.016$ ). Moreover, we found a positive association with M. coquereli (encounter rates: ER_Mc.ds) in non-degraded habitat 
(ER_Mc.ds*degr.0: $B=1.148, d f=1, p=0.019$ ), but not in degraded habitat (ER_Mc.ds*degr.1: $B=-0.087, d f=1, p=0.845 ;$ figure $48 b)$.

The following log-linear model fitted best to dry season encounter rates of $M$. berthae and explained $10.74 \%$ of total variance in encounter rates:

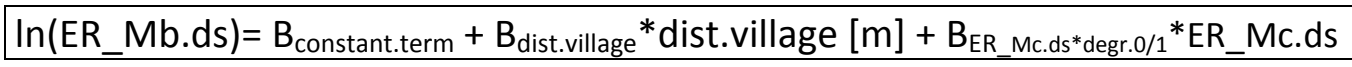

Dry season model predictions per transect for non-degraded habitat resulted from $\ln \left(E R \_M b . d s\right)=-2.075+0.211 *$ dist.village $[\mathrm{m}]+1.148 * E R \_M c . d s$ and for degraded habitat from $\ln \left(E R \_M b . d s\right)=-2.075+0.211 *$ dist.village $[m]+(-0.087) * E R \_M c . d s$ (figure 48a).
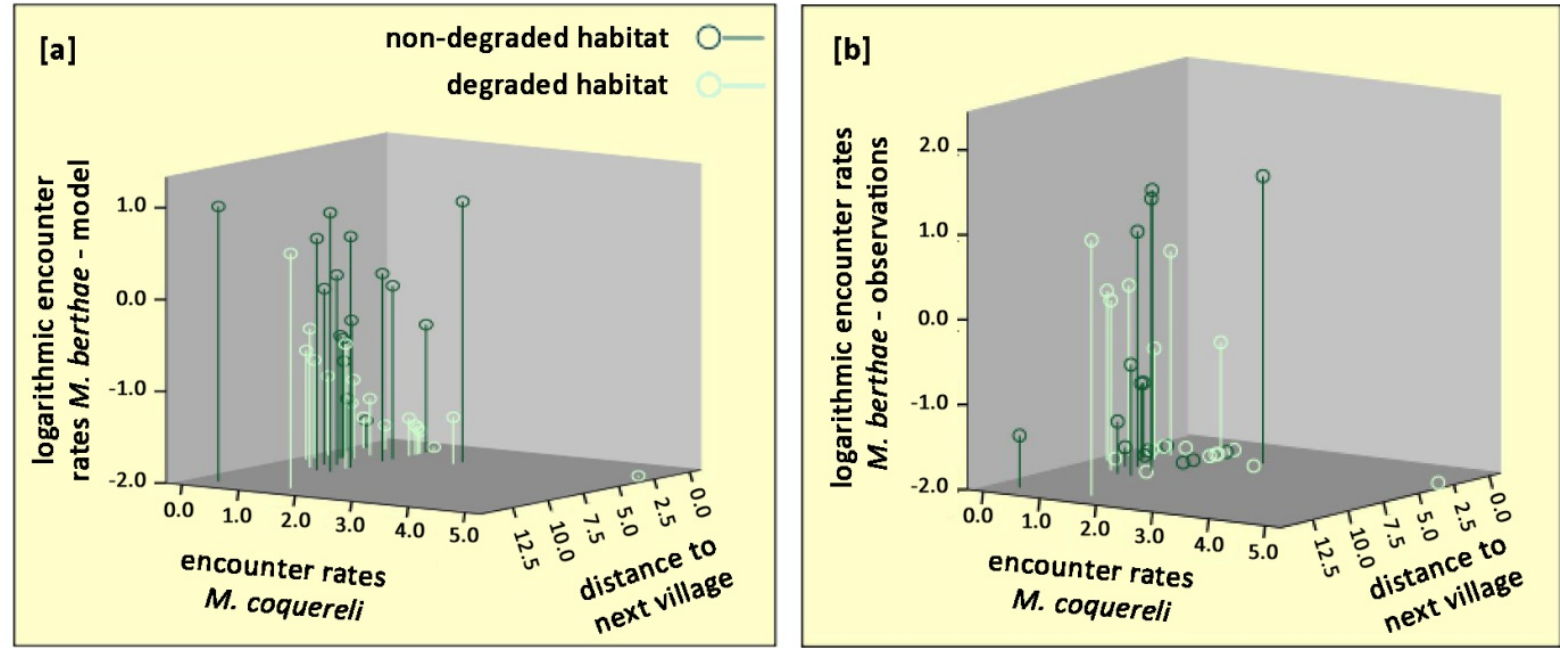

Fig. 48: [a] Log-linear model fitted to [b] observed dry season distribution of $M$. berthae in non-degraded $(n=16)$ and degraded habitat $(n=18)$

During the rainy season, we encountered more $M$. berthae in non-degraded than in degraded habitat (degrad.0: $B=2.007, d f=1, p=0.005$ ), whereas the population distribution was independent of the distance from villages. Independent of habitat degradation, $M$. berthae's encounter rates were positively related to those of $M$. coquereli (n.s. trend: ER_Mc.rs: $B=0.681, d f=1, p=0.094$; figure $49 b$ ).

The following best fitting log-linear model accounted for $13.05 \%$ of the total variance in encounter rates:

$$
\ln \left(E R \_M b . r s\right)=B_{\text {constant.term }}+B_{\text {degrad.0/1 }}+B_{\text {ER_Mc.rs }}{ }^{*} E R \_M c . r s
$$

Rainy season predictions (figure 49a) for non-degraded habitat were calculated per transect from In(ER_Mb.rs) $=-2.289+2.007+0.681 * E R \_M c . r s$ and for degraded habitat from $\ln \left(E R \_M b . r s\right)=-2.289+0+0.681 * E R \_M c . r s$. 

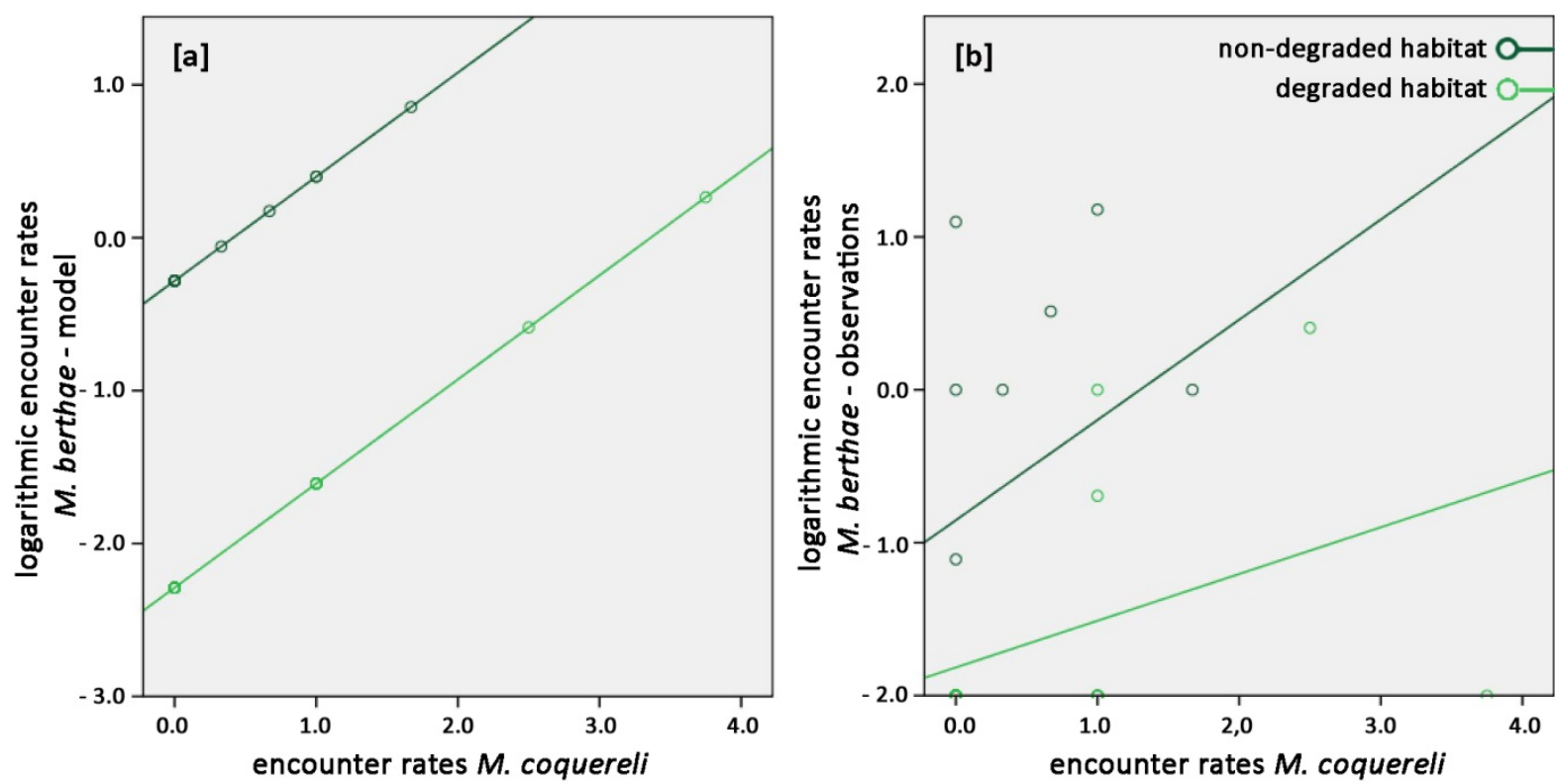

Fig. 49: [a] Log-linear model describing [b] observed rainy season distribution of $M$. berthae; as variances in $M$. coquereli encounter rates were low in both seasons (five different encounter rate values in non-degraded $(n=11)$, and four in degraded habitat $(n=14))$, model predictions of numerous transects overlap.

M. murinus distribution during the dry season regionally varied, with significantly lower encounter rates (ER_Mm.ds) in the northern forest part Ambadira compared to other forest regions (region: $B=-2.117, d f=1, p=0.021$; figures $50 a, b)$. Moreover, $M$. murinus was negatively associated with $M$. coquereli in non-degraded habitat (ER_Mc.ds: $B=-1.479, d f=1$, $p=0.023)$, and encounter rates decreased non-significantly with increasing distance from a village $(B=-0.131, d f=1, p=0.079)$.

Model predictions and observations for Kirindy Forest are separately depicted in figures 51a, b. Predictions were calculated for all regions and for non-degraded and degraded habitat respectively, with coefficients given in table 9 . The dry season model explained $10.25 \%$ of the total variance in $M$. murinus' encounter rates:

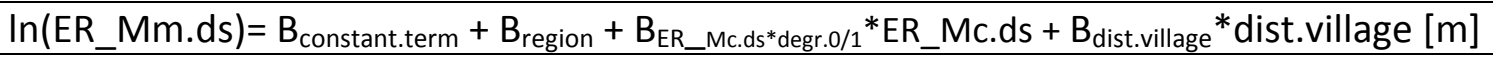

Table 9: Coefficients of dry season model for M. murinus

\begin{tabular}{|c|l|c|c|c|c|}
\hline \multirow{2}{*}{ B $_{\text {constant.term }}$} & \multicolumn{1}{|c|}{ region } & B $_{\text {region }}$ & B $_{\text {ER_Mc.ds*degr.0 }}$ & B $_{\text {ER_Mc.ds*degr.1 }}$ & B $_{\text {dist.village }}$ \\
\hline \multirow{3}{*}{3.130} & Ambadira & -2.117 & & & \\
\cline { 2 - 3 } & Corridor & -0.413 & \multirow{3}{*}{-1.479} & -0.675 & -0.131 \\
\cline { 2 - 4 } & Kirindy & -0.876 & & & \\
\cline { 2 - 4 } & RSA & 0 & & & \\
\hline
\end{tabular}



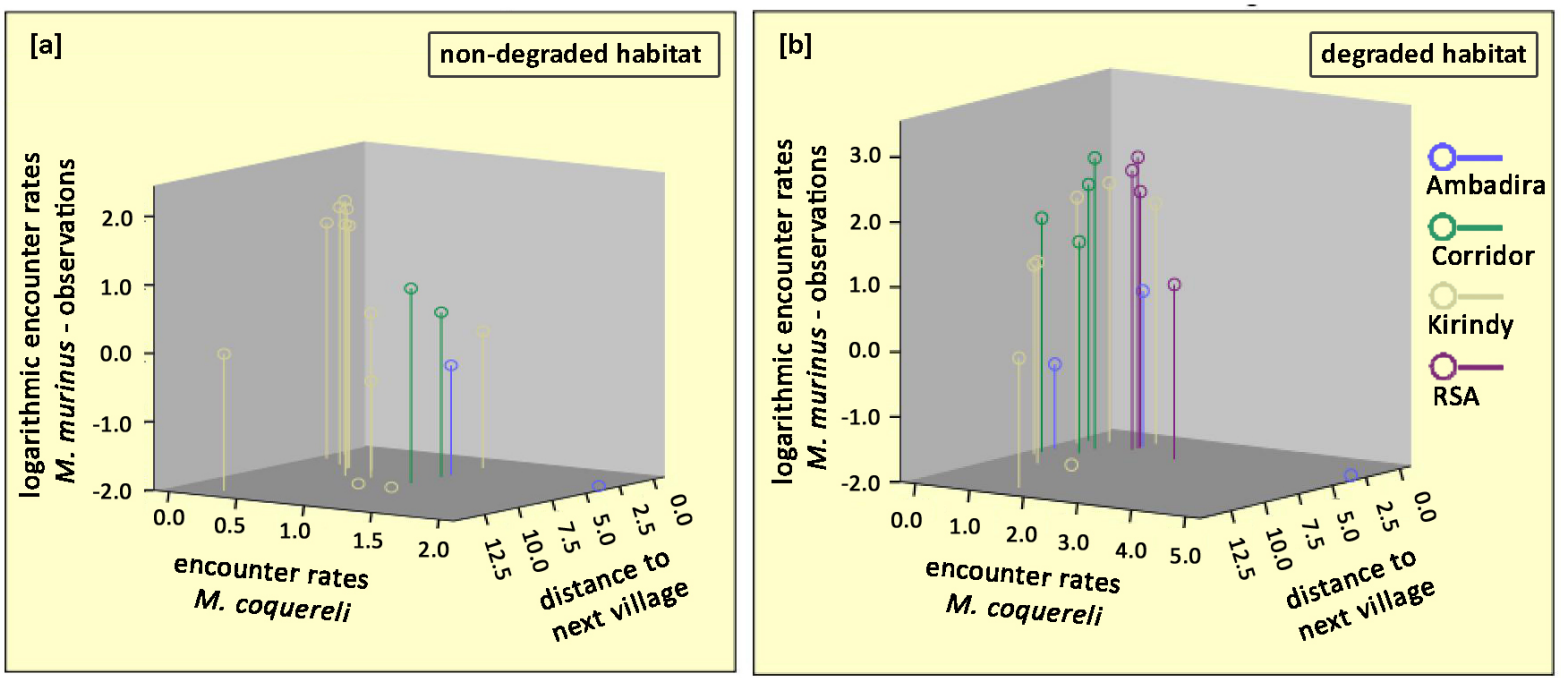

Fig. 50: Observed dry seasons distribution of M. murinus over all regions in [a] non-degraded $(n=16)$ and [b] degraded habitat $(n=18)$

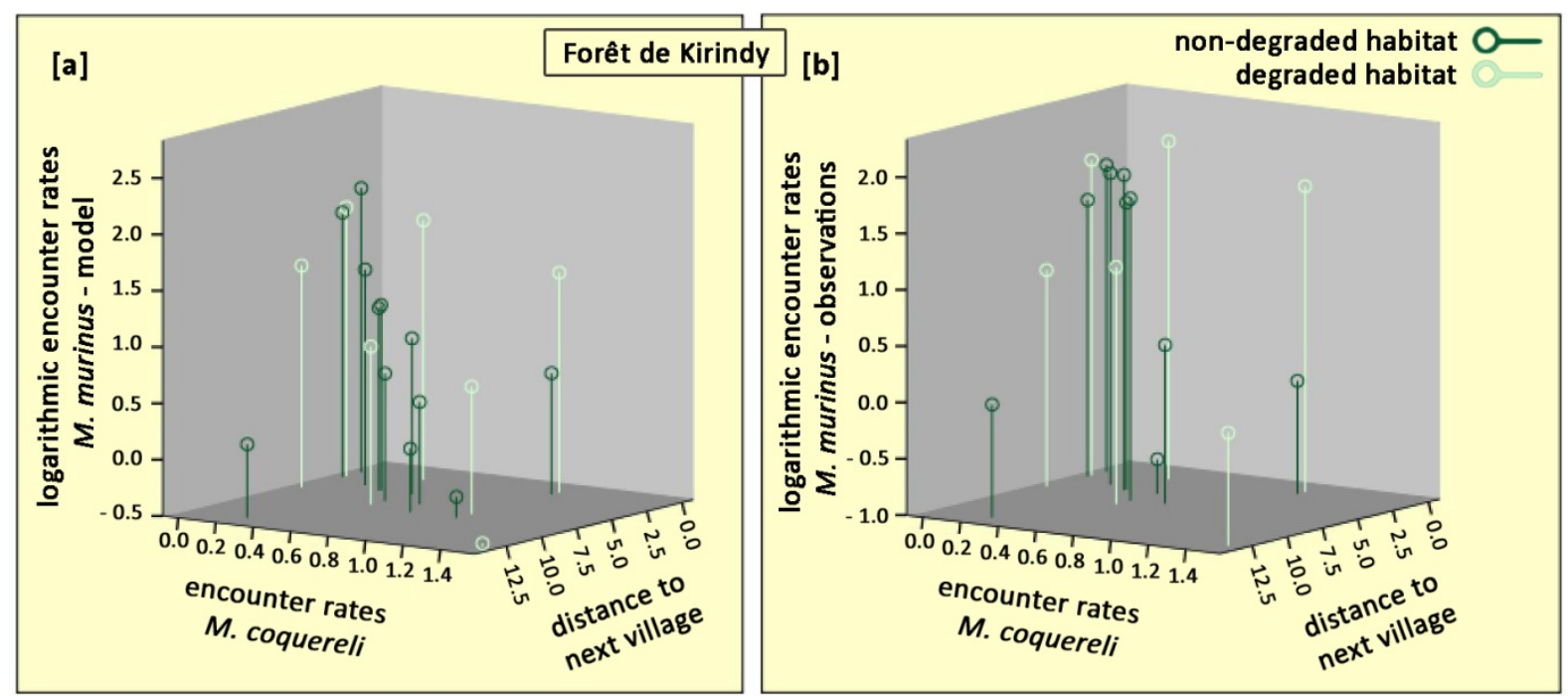

Fig. 51: [a] Log-linear model fitted to [b] observed dry season distribution of $M$. murinus in Kirindy forest in non-degraded $(n=12)$ and degraded habitat $(n=7)$

During the rainy season we found a corresponding, but non-significant, trend towards lower encounter rates (ER_Mm.rs) in Ambadira compared to the other forest regions ( $B=-1.841$, $d f=1, p=0.096$; figures $52 a, b)$. Rainy season data yielded a negative interspecific association with $M$. coquereli in non-degraded habitat only $(B=-1.841, d f=1, p=0.004)$, but vicinity to a village did not have any effect. Rainy season predictions were based on coefficients given in table 10 and the rainy season model term accounted for $9.11 \%$ of total variance:

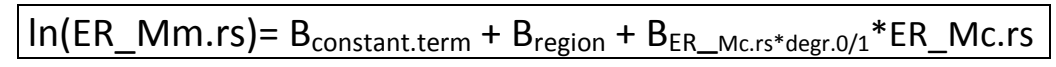


Table 10: Coefficients of rainy season model for M. murinus

\begin{tabular}{|c|c|c|c|c|}
\hline B $_{\text {constant.term }}$ & region & B $_{\text {region }}$ & B $_{\text {ER_Mc.rs*degr.0 }}$ & B $_{\text {ER_Mc.rs*degr.1 }}$ \\
\hline \multirow{4}{*}{1.878} & Ambadira & -2.128 & & \\
\cline { 2 - 3 } & Corridor & -0.320 & \multirow{3}{*}{-1.841} & 0.021 \\
\cline { 2 - 3 } & Kirindy & -0.077 & & \\
\cline { 2 - 3 } & $\mathrm{RSA}$ & 0 & & \\
\hline
\end{tabular}
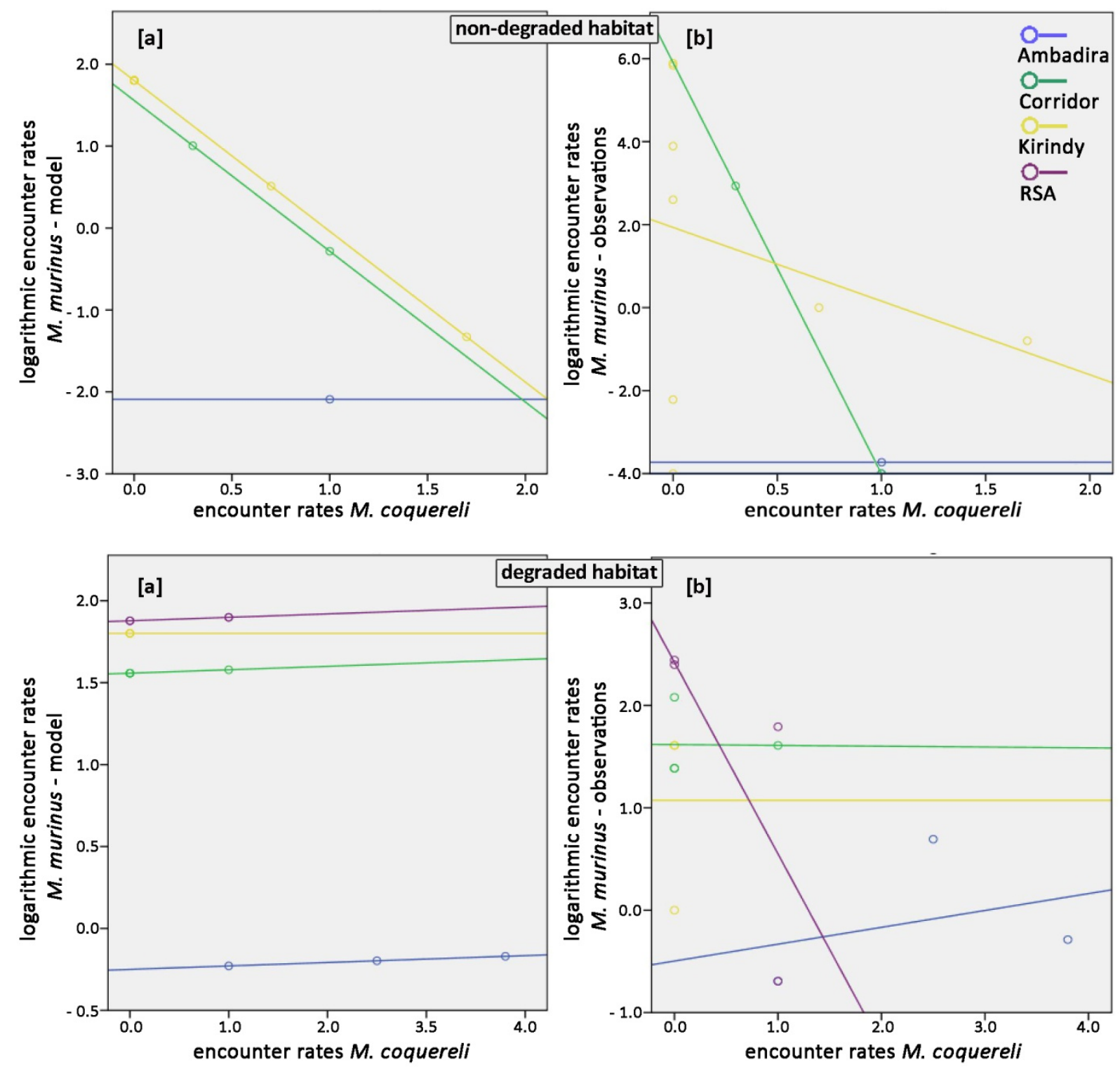

Fig. 52: [a] Log-linear model describing [b] observed rainy season distribution of $M$. murinus over all regions, in non-degraded ( $n=11$; top of fig. 52 ) and degraded habitat ( $n=14$; bottom graphs in fig. 52 ) respectively. 


\section{Determinants of local mouse lemur distribution: Interindividual modeling}

\section{Intraspecific distribution of individuals within sections}

The two species of Microcebus did not only differ in the number of sections they were found on, but also in the number of sections on which intraspecific pairs were observed. Dry season surveys revealed that more than one $M$. berthae individual was present on seven of 18 sections, i.e. $39 \%$ of the sections colonized at all by the species (corresponding to 17 transects in figure 14a), and all those seven had intraspecific pairs within $100 \mathrm{~m}$. On two of those sections (both in Kirindy forest) we found more than three pairs (figure 53a +54 ).
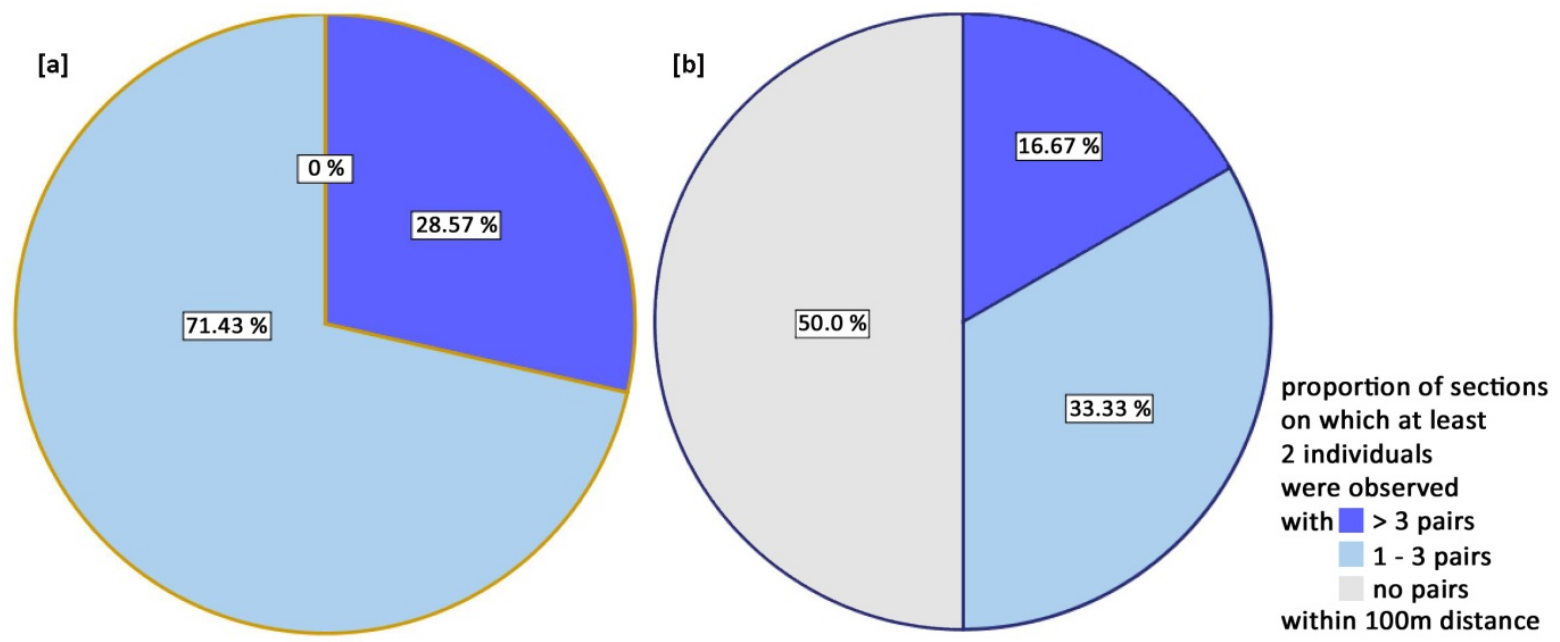

Fig. 53: Proportions of sections on which $M$. berthae formed intraspecific pairs within $100 \mathrm{~m}$ distance in [a] dry $(n=7)$ and $[b]$ rainy season $(n=6)$

Interindividual distances between observed individuals were lower than expected from random distribution on section LS $9.2(p=0.001, n=4)$, but there was no difference on section $\mathrm{K} 05$ ( $p=0.567, n=8$; figure 54). 

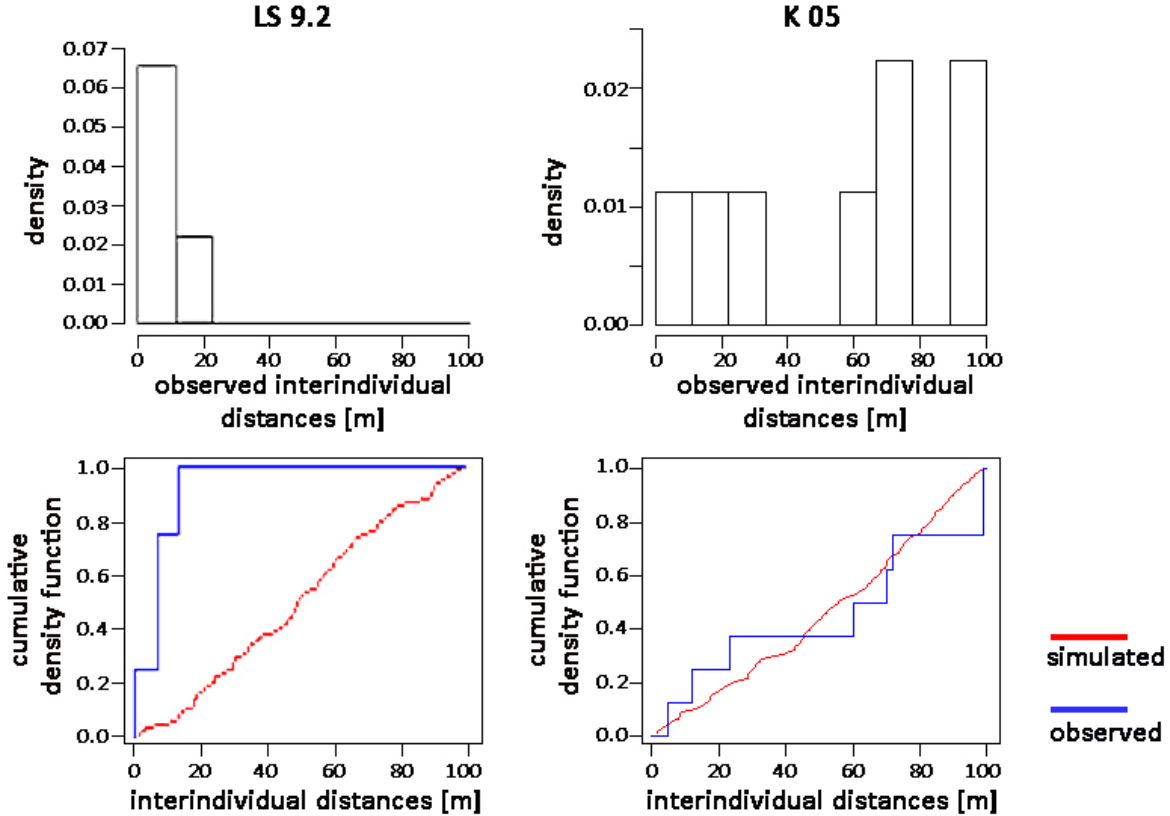

Fig. 54: Microcebus berthae intraspecific pairs (dry season) on sections LS 9.2 with individuals stronger associated in space than expected, and K 05 with interindividual distribution as expected by chance

During rainy season surveys, we encountered more than one $M$. berthae individual within a run on $55 \%$ of all sections on which the species was present (i.e. six of 11 sections colonized,

A 04
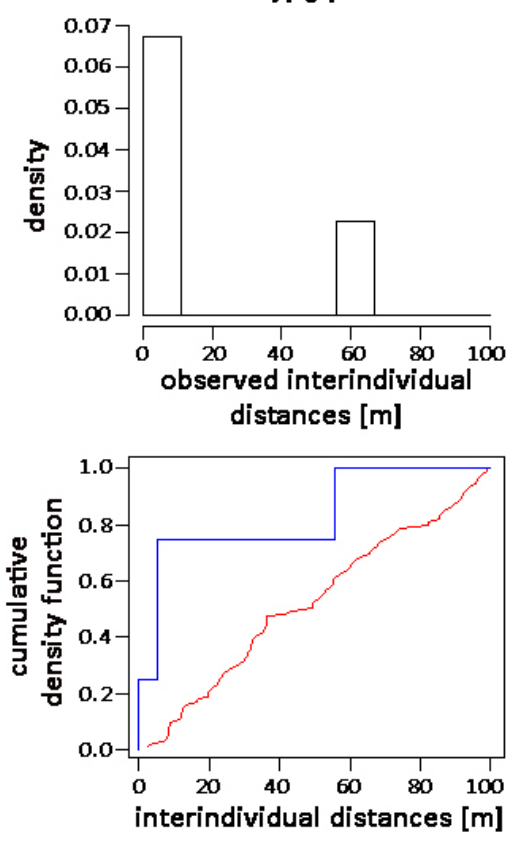

corresponding to 10 sections in figure 14b). Three of those sections had any intraspecific pairs of individuals within a distance of $100 \mathrm{~m}$, and more than three pairs were observed on one section (figure 53b). On the respective section A 04 (in Ambadira), observed individuals were found in positive association $(p=0.017, n=4$; figure 55$)$.

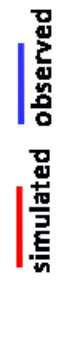

Fig. 55: Section A04 (rainy season) with M. berthae individuals stronger associated in space than expected by chance

Conspicuously, positive associations of $M$. berthae individuals were indicated only on two sections with a low number of intraspecific pairs available for analysis, whereas there were no deviations from random associations on sections with a higher number of short-distance pairs. 
More than one M. murinus individual within a run were observed on $72 \%$ of the 29 sections, on which we encountered the species during our dry season surveys (23 of 32 sections; corresponding to 29 transects in figure 15a). Intraspecific pairs within $100 \mathrm{~m}$ were observed on 22 of those sections, and 15 sections had more than three pairs (figure 56a).
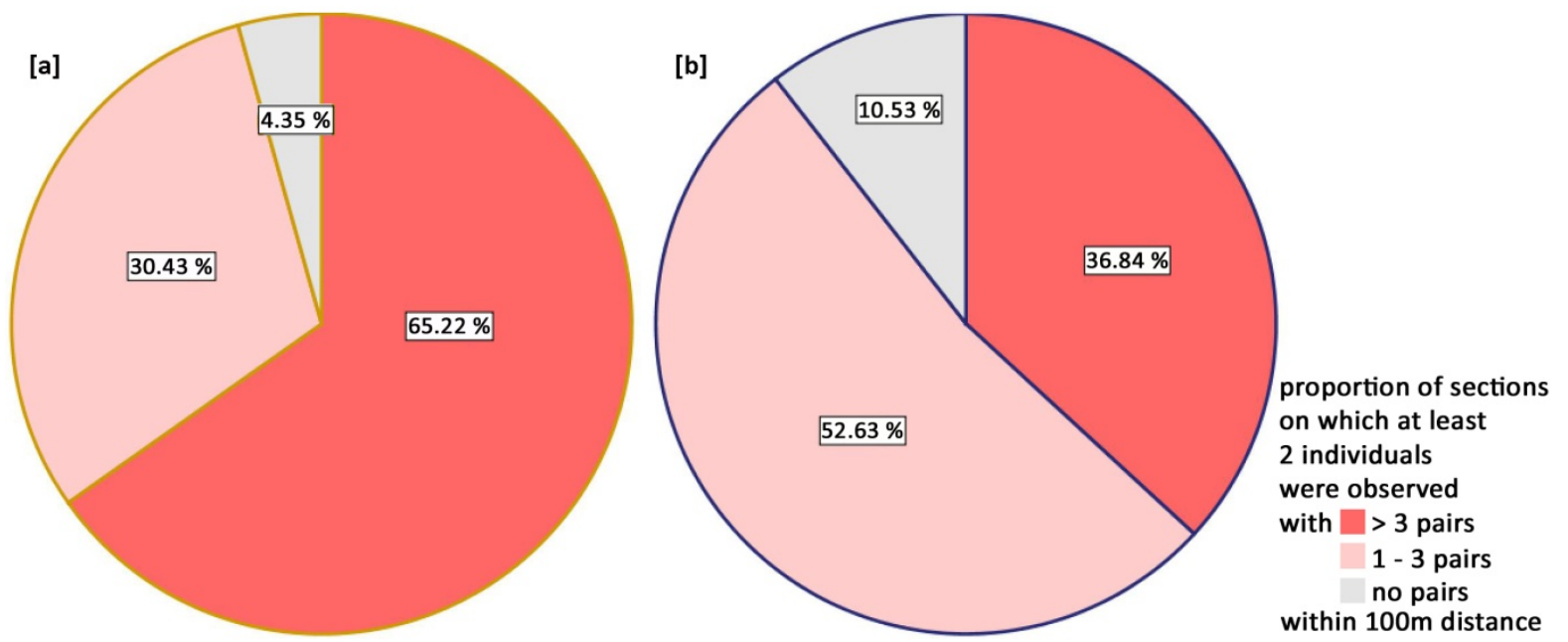

Fig. 56: Proportion of sections on which $M$. murinus formed intraspecific pairs within $100 \mathrm{~m}$ distance in [a] dry $(n=23)$ and $[b]$ rainy season $(n=19)$

On two of those sections, one situated in Kirindy forest (N5(B1-B21): $p=0.041, n=16)$, the other in the corridor (Cor SW: $p=0.017, n=6$; both in figure 57), we found individuals in closer spatial associations than would be expected by chance.
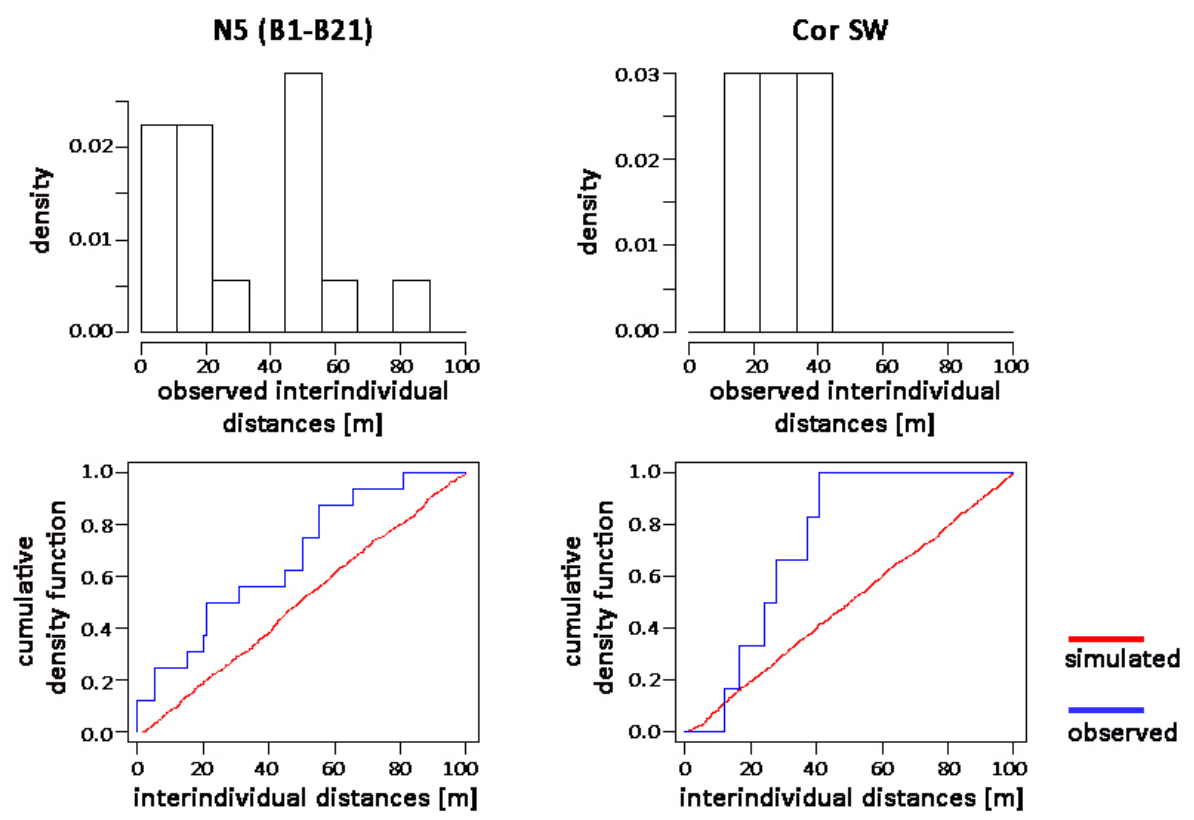

Fig. 57: Microcebus murinus intraspecific pairs (dry season) on sections N5 (B1-B21) and Cor SW with individuals closer associated in space than predicted by random distribution

However, no other section indicated the intraspecific distribution of $M$. murinus individuals to deviate from random associations (RSA S02: $p=0.848, n=44$; RSA N02: $p=0.524, n=21 ; R S A$ S01: $p=0.403, n=36$, Cor CS: $p=0.445, n=28 ; C N ~ 3.2: p=0.104, n=21 ; L S$ 2.1: $p=0.613, n=11 ; K$ 
04: $p=0.311, n=7 ; N$ 2.1: $p=0.519, n=6$; Cor SE: $p=0.786, n=6 ; K 11: p=0.842, n=6$; Kir $W$ : $p=0.294, n=5$; CS7(U7-A7): $p=0.341, n=4)$. Figure 58 exemplarily show two examples (RSA S02 and RSA N02) that had a high number of intraspecific pairs, even though these results most likely represent a type I error.

During rainy season surveys we found more than one M. murinus individuals on 19 sections, or $83 \%$ of all sections on which the species was present (23 sections, which corresponds to 22 transects in figure 15b). Intraspecific pairs within 100m were observed on 17 of those 19 sections and on seven we observed more than three pairs (figure 56b). On section N 2.1, observed interindividual distances were lower than expected from random distribution ( $p=0.024, n=6)$, but no differences emerged on any other section ( $C N$ 2.1: $p=0.101, n=54 ; C N$ 3.2: $p=0.749, n=35 ;$ RSA S02: $p=0.168, n=10$; Cor CS: $p=0.673, n=5$; Cor SE: $\mathrm{p}=0.639, \mathrm{n}=4 ; \mathrm{N} 5(\mathrm{~B} 1-\mathrm{B} 21)$ : $\mathrm{p}=0.846, \mathrm{n}=4)$. Figure 59 represents $\mathrm{N} 2.1$ with positive intraspecific association of individuals and exemplarily shows two of the sections with high numbers of intraspecific pairs, on which individuals were associated as expected by chance (CN 2.1 and CN 3.2).

RSA S02
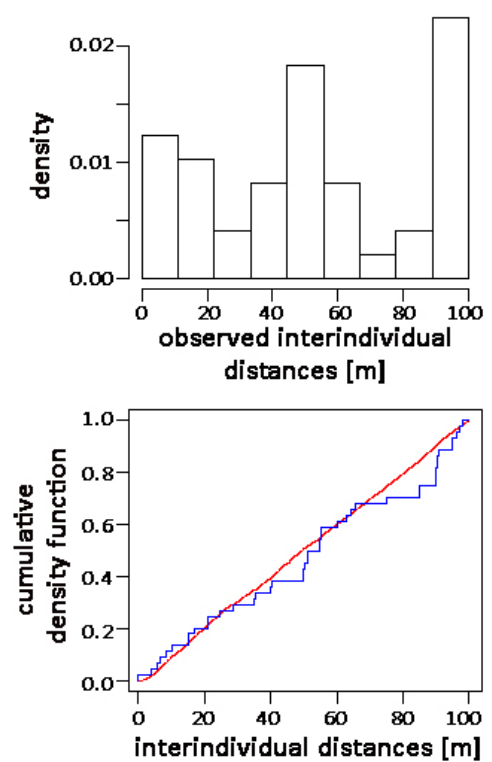

RSA N02
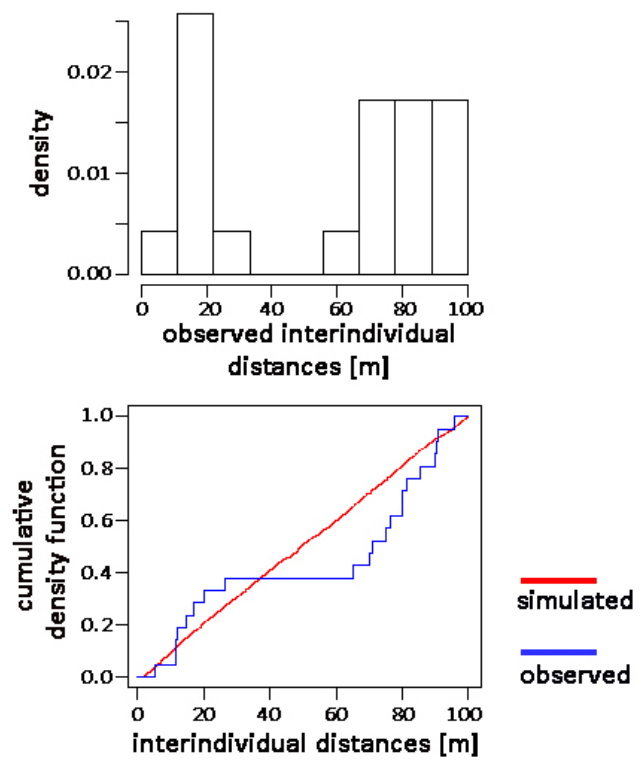

Fig. 58: Microcebus murinus intraspecific pairs (dry season) on sections RSA S02 and RSA N02 with individuals associated in space in random association 
N 2.1
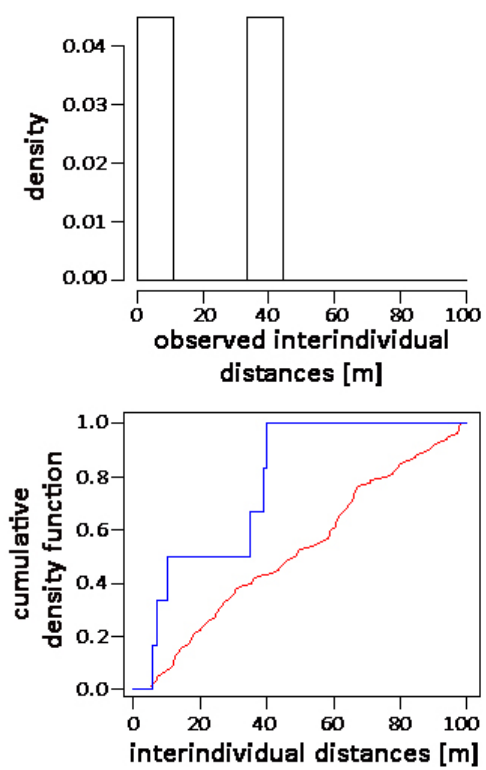

CN 2.1
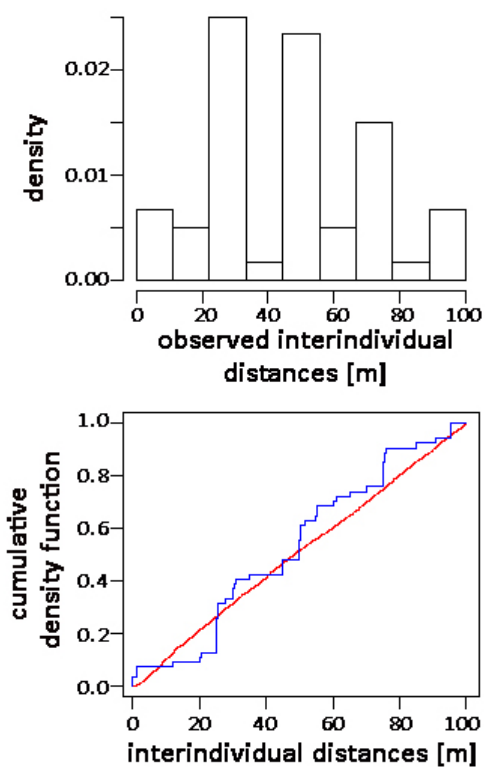

CN 3.2
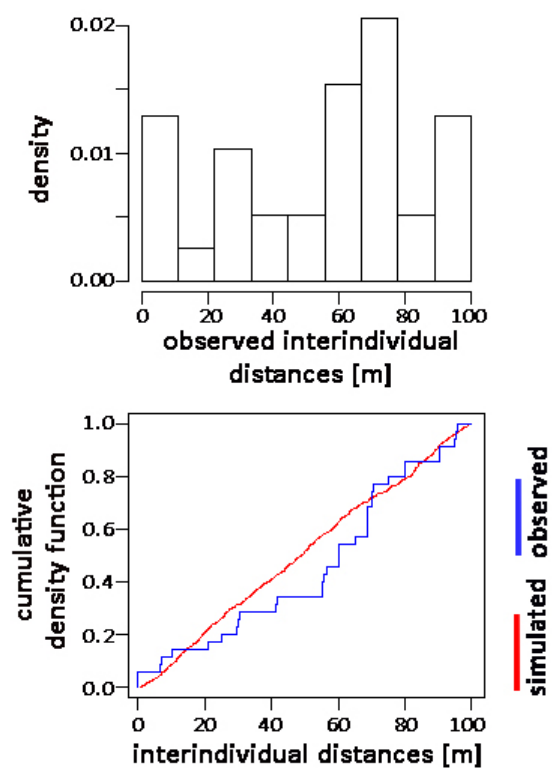

Fig. 59: Microcebus murinus intraspecific pairs (rainy season) on section N 2.1 (rainy season), where M. murinus individuals were stronger associated in space than expected by chance; in contrast, individuals on sections $\mathrm{CN}$ 2.1 and CN 3.2 were intraspecifically associated in space as expected by chance

\section{Interspecific distribution of individuals within sections}

Microcebus berthae was found in regional coexistence with $M$. murinus, as well as with $M$. coquereli and C. medius. During the dry season, we observed at least one representative of both, $M$. berthae and M. murinus within the same run on eleven of 37 sections (30\%), and on eight of 26 sections during the rainy season (31\%). Within these sections, the proportion of realized interspecific pairs did neither indicate interspecific competition between Microcebus spp. during the dry, nor during the rainy season. From M. berthae's perspective, results did not show any differences in the proportions of intraspecific pairs of individuals and pairs with $M$. murinus within 100 meters (dry season: $\chi^{2}=0.598, d f=1, p=0.439$; rainy season: $\left.\chi^{2}=1.278, d f=1, p=0.258\right)$. Considering $M$. murinus' intra- and interspecific distribution referring to $M$. berthae, we neither found differences during the dry season $\left(\chi^{2}=0.344, d f=1, p=0.558\right)$. During the rainy season we observed even more interspecific pairs than were expected $\left(\chi^{2}=4.235, d f=1, p=0.040\right)$. Based on these analyses, the level of interspecific competition between Microcebus spp. therefore does not seem to exceed that of intraspecific competition in either season. 


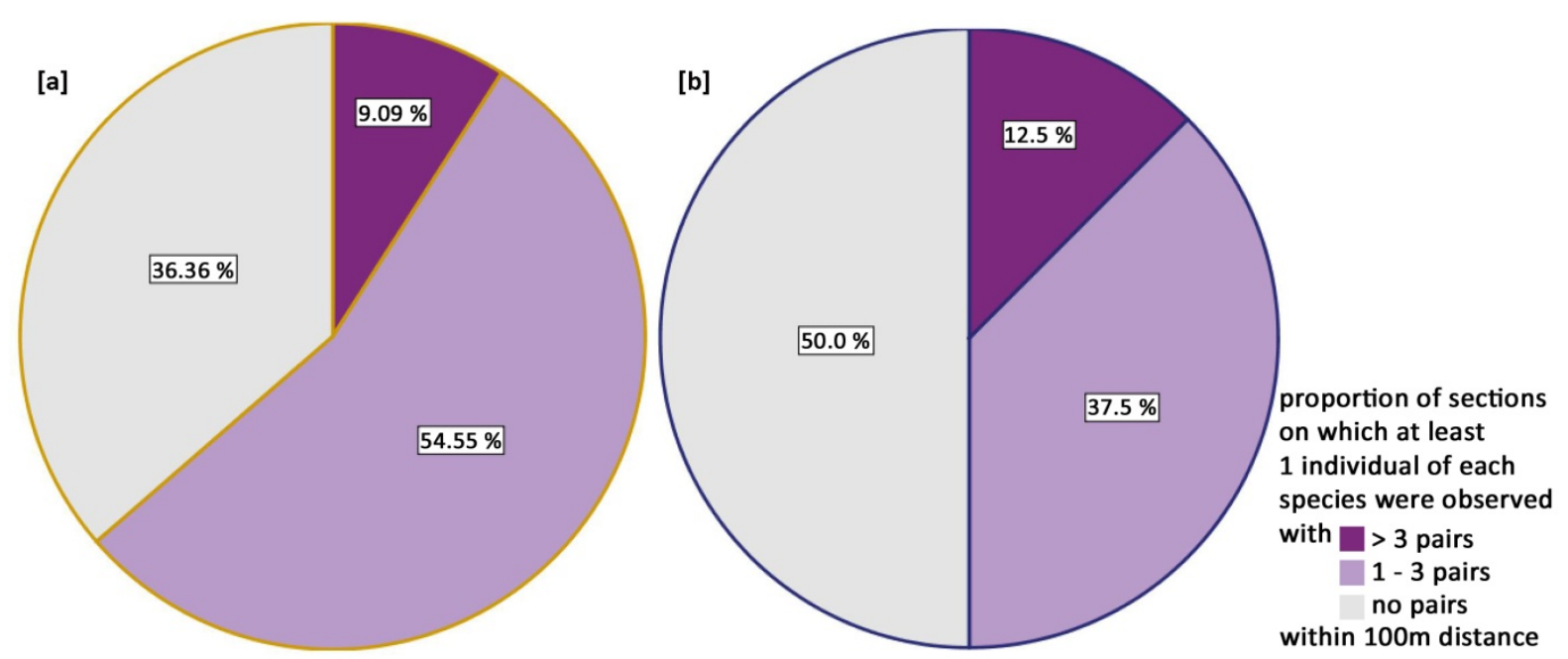

Fig. 60: Number of sections on which $M$. berthae was found in interspecific pairs with $M$. murinus during [a] dry $(n=11)$ and $[b]$ rainy season surveys $(n=8)$

More than three interspecific pairs of Microcebus spp. within a distance of $100 \mathrm{~m}$ were observed only once in either season (figure 60). Of eleven sections allowing for pair formation during the dry season, seven had any pairs within 100m (figure 60a). Interindividual associations on the one section having more than three interspecific pairs did

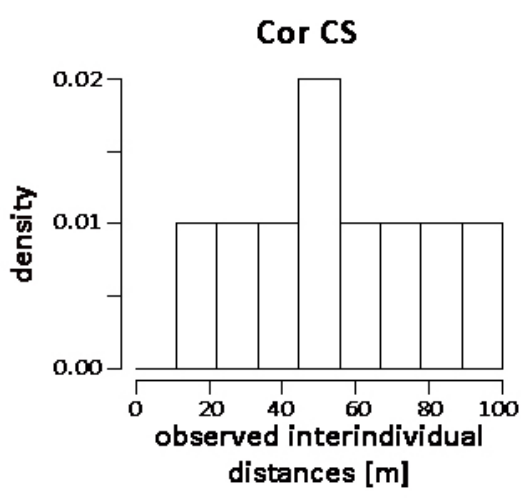
not differ from a random distribution (Cor CS: $p=0.762, n=9 ;$ figure 61).

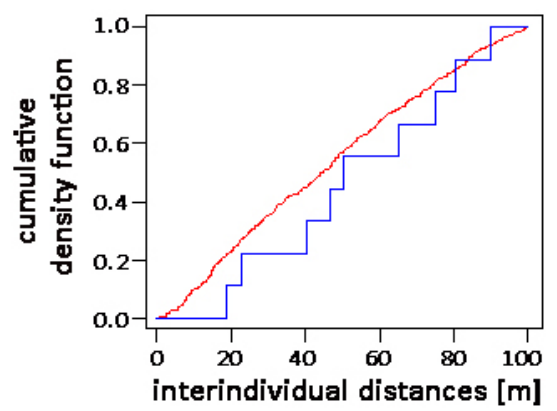

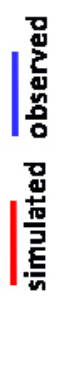

Fig. 61: Observed distances between interspecific Microcebus pairs (dry season) observed on section Cor CS were compliant with random distribution

The two mouse lemurs regionally co-occurred on $31 \%$ of sections sampled during the rainy season (eight of 26 sections), i.e. we observed at least one individual of either mouse lemur within the same run. On four of those eight sections, at least one pair occurred within $100 \mathrm{~m}$ 
(figure 60b). Interspecific distribution of individuals on the single section having more than three pairs did not differ from a random distribution (N5(B1-B21): $p=0.702, n=4$; figure 62).
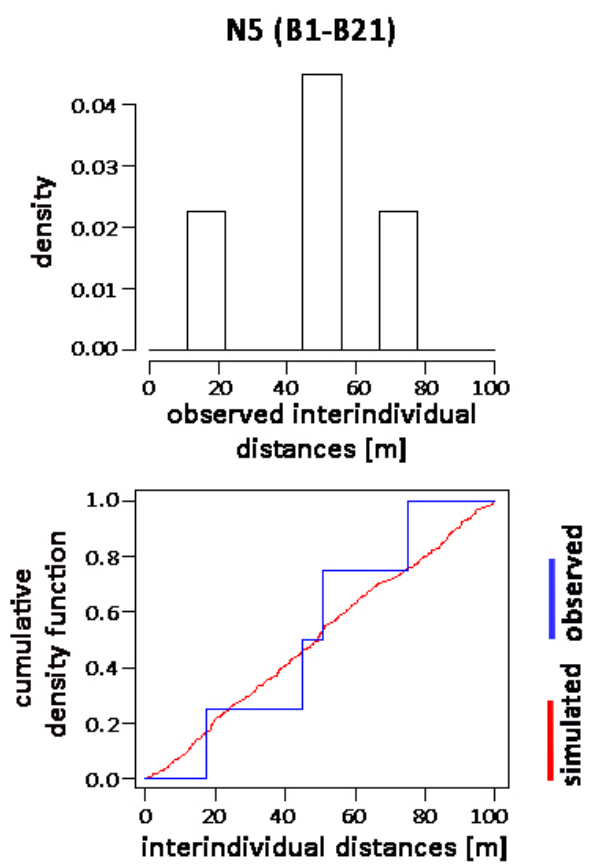

Fig. 62: Observed distances between interspecific Microcebus pairs (rainy season) observed on section N5(B1-B21) were compliant with random distribution

Individuals of both, $M$. berthae and $M$. coquereli, were observed on $19 \%$ of sections during the dry season (seven of 37 sections with minimum one individual of each species observed within a run), and five of those had pairs within 100m (figure 63a).
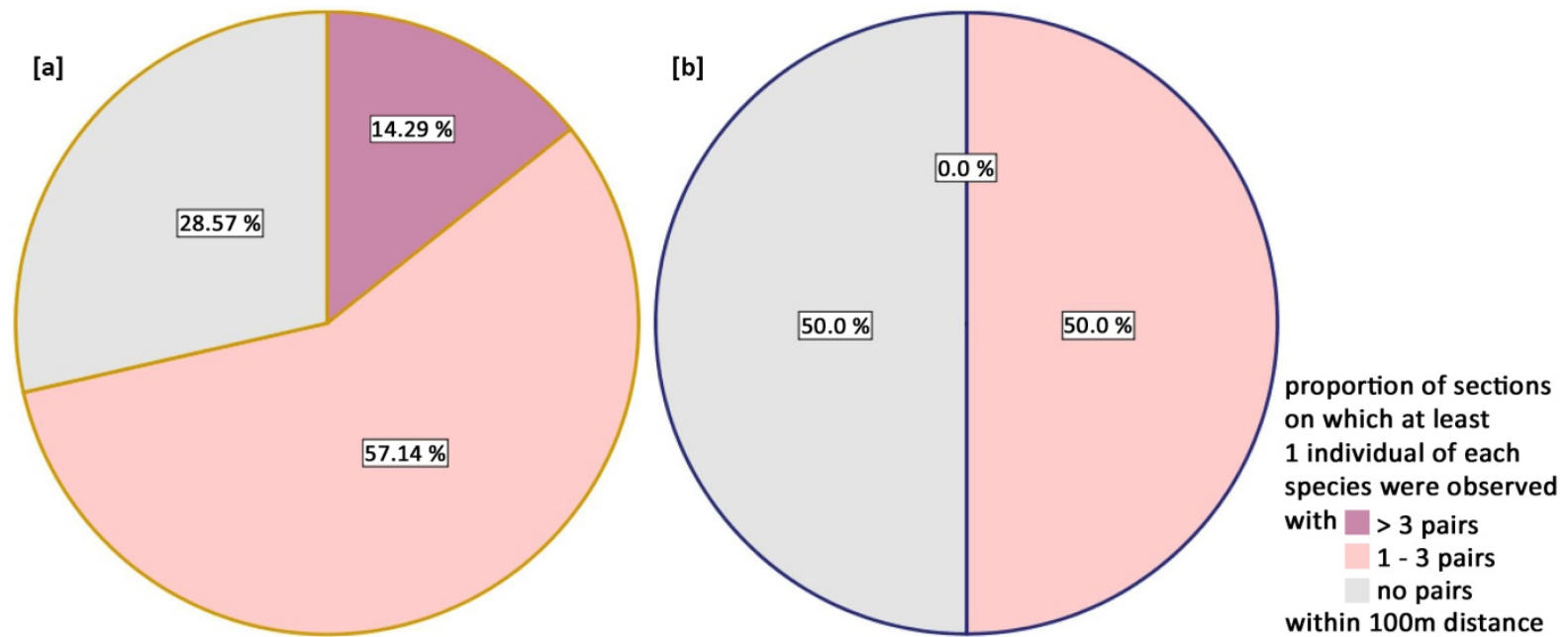

Fig. 63: Proportion of sections on which $M$. berthae was found in interspecific pairs with $M$. coquereli during [a] dry $(n=7)$ and $[b]$ rainy season surveys $(n=6)$

Within sections, no spatial association pattern emerged between $M$. coquereli and $M$. berthae. Potential and realized intra- and interspecific pairs differed neither from $M$. berthae's perspective (dry season: $\chi^{2}=0.939, \mathrm{df}=1, \mathrm{p}=0.333$; rainy season: $\chi^{2}=0.202, \mathrm{df}=1$, 
$p=0.653$ ), nor from that of $M$. coquereli (dry season: $\chi^{2}=0.079, d f=1, p=0.778$; rainy season: $\left.\chi^{2}=0.224, d f=1, p=0.636\right)$.

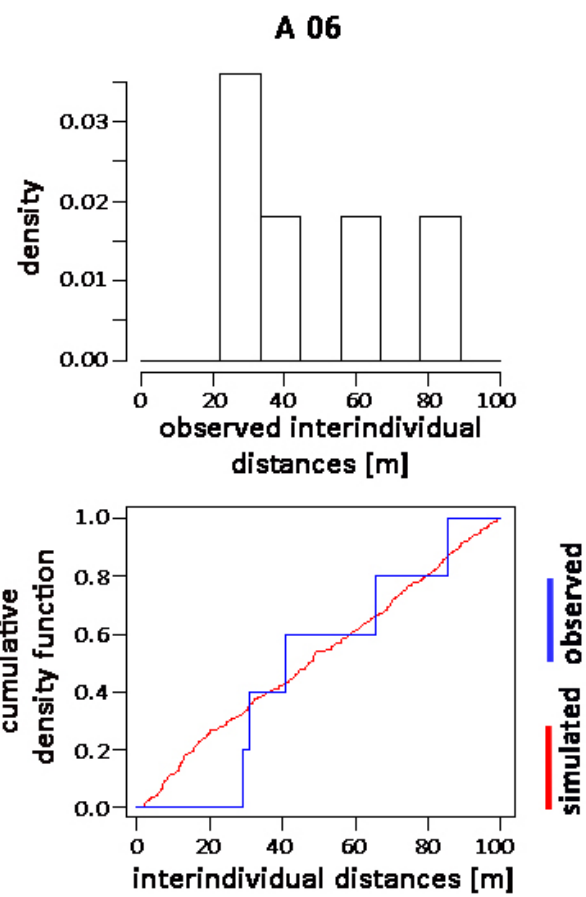

On the only one section that had more than three interspecific pairs, animals were distributed as expected by chance (A 06: $p=0.744, n=5$; fig 64).

We found at least one individual of $M$. berthae and $M$. coquereli within the same run on $23 \%$ of all sections sampled (six of 26) during the rainy season. Interspecific pairs within $100 \mathrm{~m}$ distance were found on only three of those sections and we never observed more than three pairs (figure 63b).

M. murinus individuals were found together with $M$. coquereli individuals on the same section on $24 \%$ of sections sampled during our dry season surveys (nine of 37 sections), but spatial associations with $M$. coquereli within $100 \mathrm{~m}$ distance were rarely observed. Microcebus murinus and $M$. coquereli were closely associated within $100 \mathrm{~m}$ on two sections during the dry season (figure 65a). Only during the dry season, M. murinus formed considerably fewer interspecific short-distance pairs with $M$. coquereli than expected $\left(\chi^{2}=22.727, d f=1, p=0.000\right.$; rainy season: $\left.\chi^{2}=0.951, d f=1, p=0.329\right)$. Limitation of spatial avoidance to the scarce dry season upheld when comparing potential and realized pairs $M$. coquereli formed intraspecifically and with $M$. murinus (dry season: $\chi^{2}=8.971, d f=1, p=0.003$; rainy season: $\chi^{2}=0.917, d f=1, p=0.338$ ). 


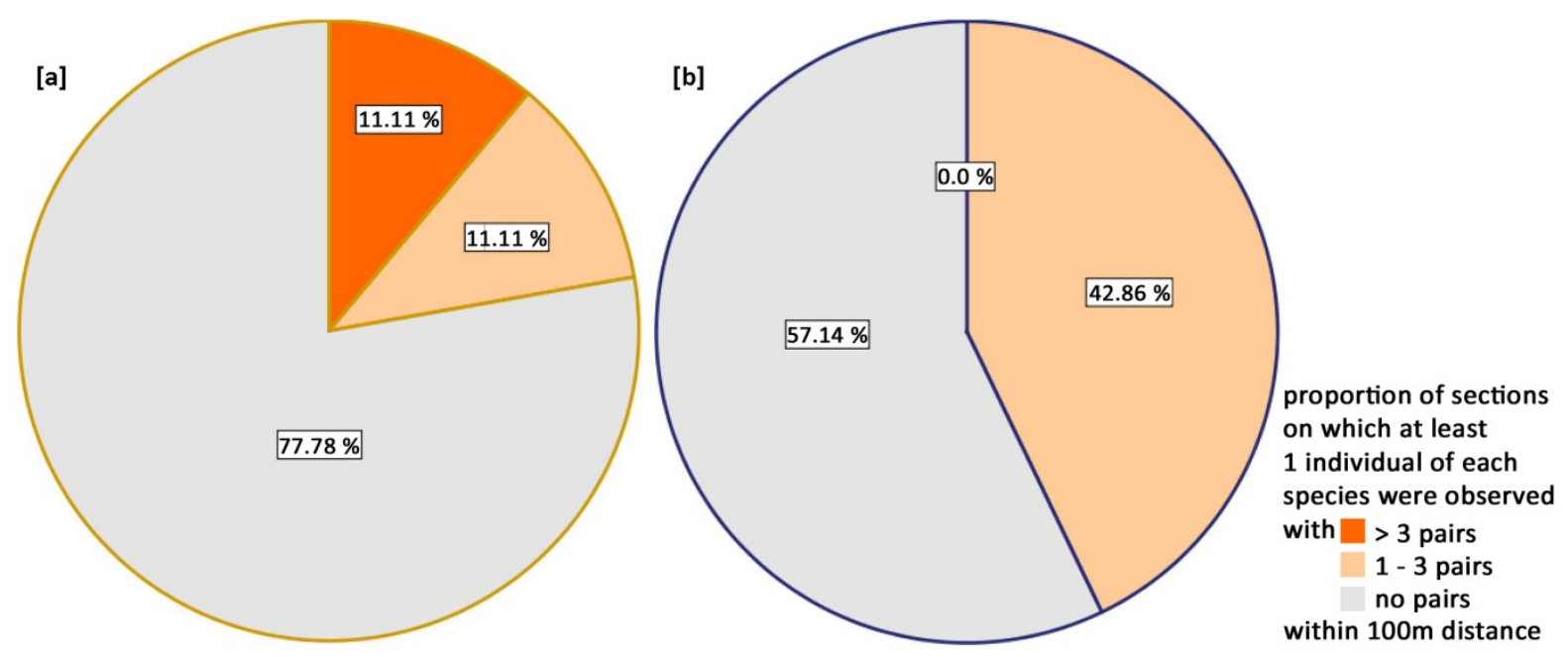

Fig. 65: Proportion of sections on which M. murinus was found in interspecific pairs with $M$. coquereli during [a] dry $(n=9)$ and $[b]$ rainy season $(n=7)$

We observed more than three pairs on a section only once during the dry season, and interspecific associations did not differ from random distribution (RSA N02: $p=0.635, n=4$; figure 66).

RSA N02
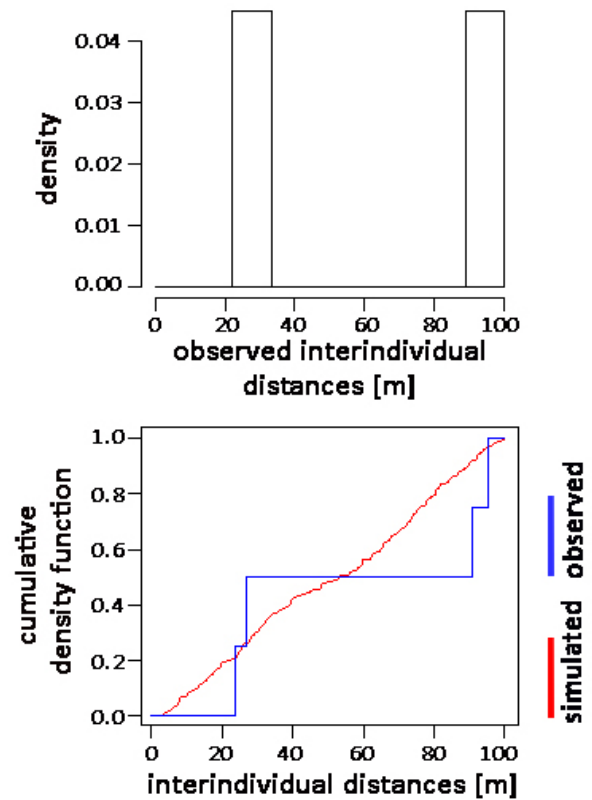

Fig. 66: Intraspecific spatial association of $M$. murinus and $M$. coquereli on section RSA N02 during the dry season as expected by chance

On $27 \%$ of sections (seven of 26 ) sampled within rainy season surveys we detected at least one $M$. murinus and one $M$. coquereli within the same run. Less than half of those (three of seven) had any pairs within $100 \mathrm{~m}$, and more than three pairs were never observed (figure 65b).

Comparing the proportions of close interspecific associations of $M$. coquereli with either of the mouse lemurs, we observed more individuals below $100 \mathrm{~m}$ distance from $M$. 
berthae individuals and fewer in association with $M$. murinus than expected during the dry season $\left(\chi^{2}=9.332, d f=1, p=0.002\right)$, whereas rainy season data did not indicate any differences $\left(\chi^{2}=0.249, \mathrm{df}=1, \mathrm{p}=0.618\right)$. Thus, $M$. coquereli appears to disproportionally affect $M$. murinus as an intraguild-predator during the scarce dry season.

Finally, we observed individuals of both, $M$. berthae and $C$. medius, within the same run on $31 \%$ (eight of 26) of sections sampled during the rainy season. Almost all of those sections (seven of eight) had pairs within $100 \mathrm{~m}$ (figure 67). We found individuals of $M$. berthae and $C$. medius in positive interspecific association during the rainy season. Microcebus berthae individuals were found more frequently in short distance to $C$. medius than was predicted for sections based on respective inter- and intraspecific long-distance proportions $\left(\chi^{2}=4.636\right.$, $d f=1, p=0.031$ ). Close spatial association was also found from the perspective of $C$. medius, i.e. we found a greater proportion of inter- and a smaller of intraspecific pairs than found in pairs of individuals $500 \mathrm{~m}$ or more apart each other $\left(\chi^{2}=16.530, \mathrm{df}=1, \mathrm{p}=0.000\right)$.

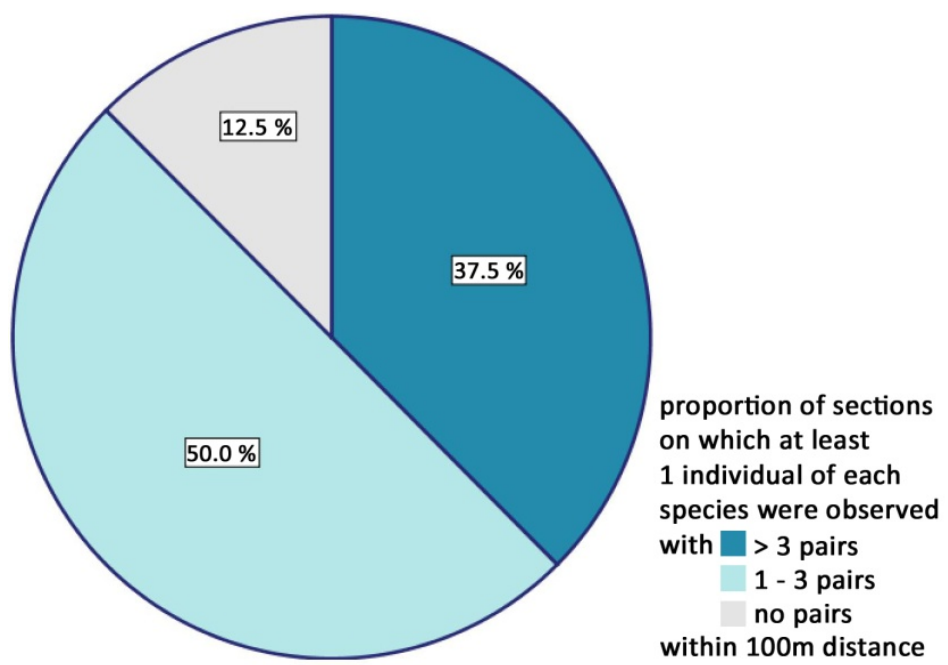

Fig. 67: Proportion of sections on which M. berthae was found in interspecific pairs with C. medius $(n=8)$

In one of three sections with more than three interspecific pairs, observed distances exceeded the simulated ones ( $A$ 06: $p=0.087, n=7$ ), but no difference between observed and simulated distributions emerged on the other two sections (A 04: $p=0.923, n=8 ; C N$ 9.2: $p=0.401, n=5 ;$ all in figure 68). 
A 06
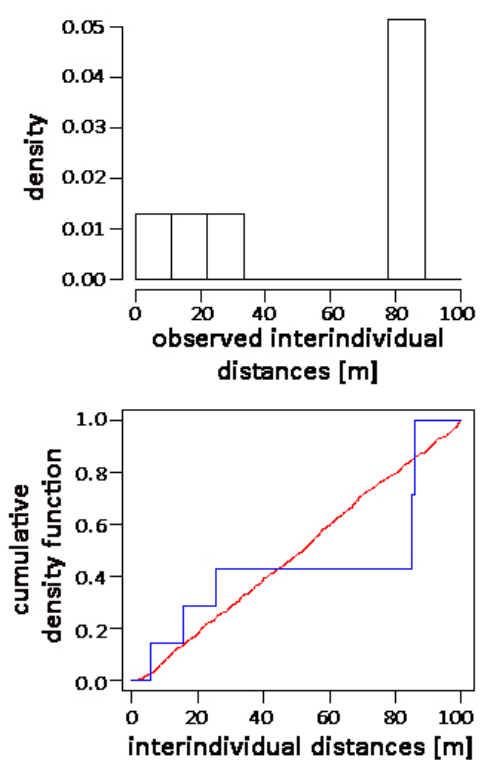

A 04
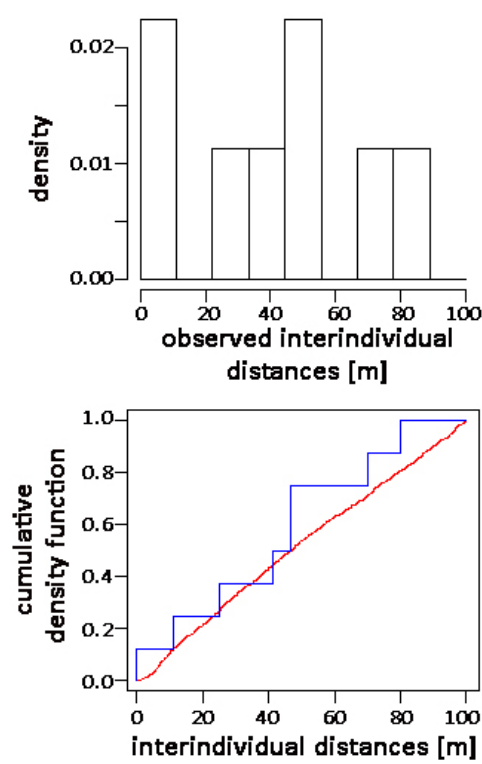

CN 9.2
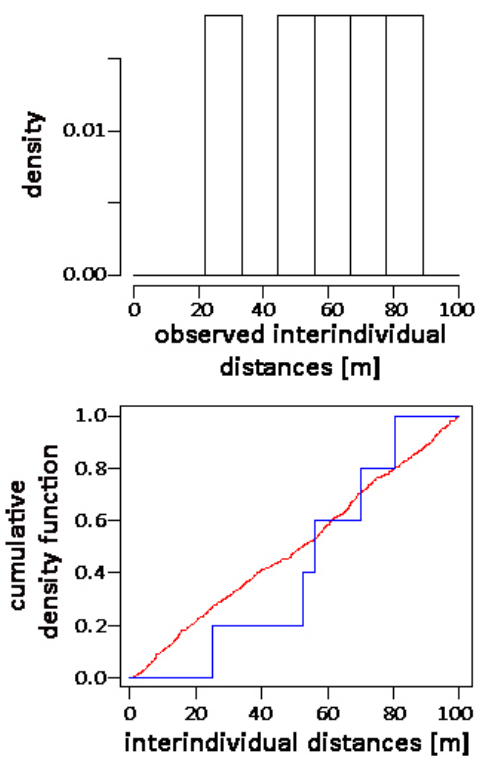

Fig. 68: Microcebus berthae interspecific pairs with C. medius (rainy season) on section A 06 with individuals stronger associated in space than expected by chance; on sections A 04 and $\mathrm{CN} 9.2$ individuals were interspecifically associated in space as expected by chance

Of all sections sampled during the rainy season, $65 \%$ (17 of 26 sections) had at least one $M$. murinus and one $C$. medius encountered within the same run. Fourteen of these sections had any pairs within $100 \mathrm{~m}$ (figure 69 ). Within sections, we observed fewer interspecific short-distance pairs of $M$. murinus and $C$. medius individuals than were predicted based on long-distance pairs $\left(\chi^{2}=73.317, \mathrm{df}=1, \mathrm{p}=0.000\right)$, indicating spatial avoidance of $C$. medius by M. murinus individuals. Correspondingly, C. medius realized fewer short-distance pairs with M. murinus than expected $\left(\chi^{2}=21.063, d f=1, p=0.000\right)$.

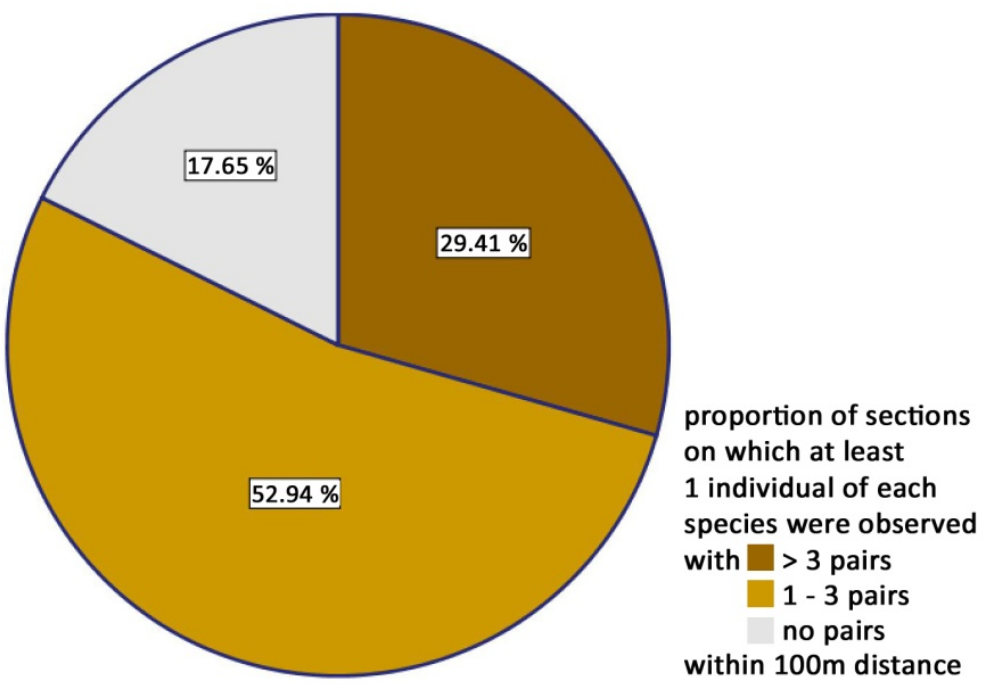

Fig. 69: Proportion of sections on which M. murinus was found in interspecific pairs with $C$. medius $(\mathrm{n}=17)$ 
On five sections we observed more than three pairs. Whilst one section indicated stronger interspecific clumping of individuals (NN 7: $p=0.020, n=7$ ), individuals of another section were interspecifically more dispersed than expected in a random distribution (Kir W:
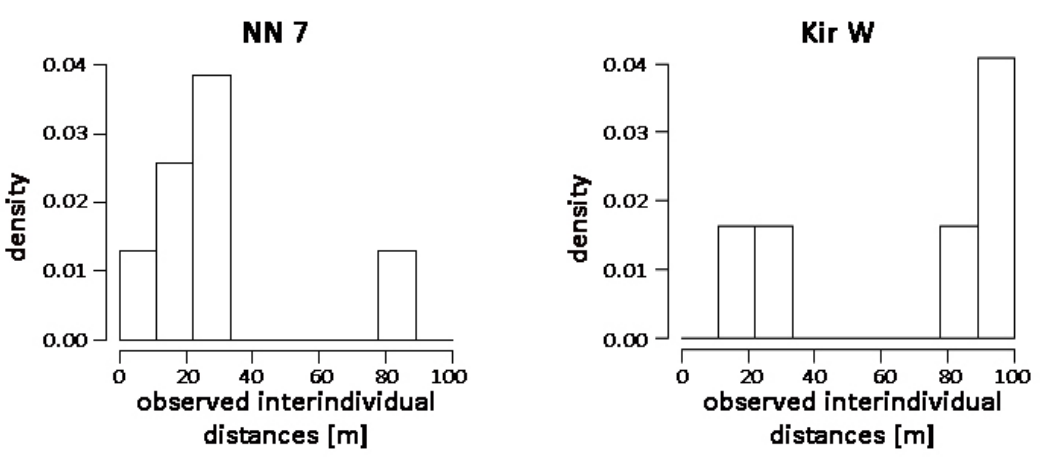

$\mathrm{p}=0.005 ; \quad \mathrm{n}=11 ;$
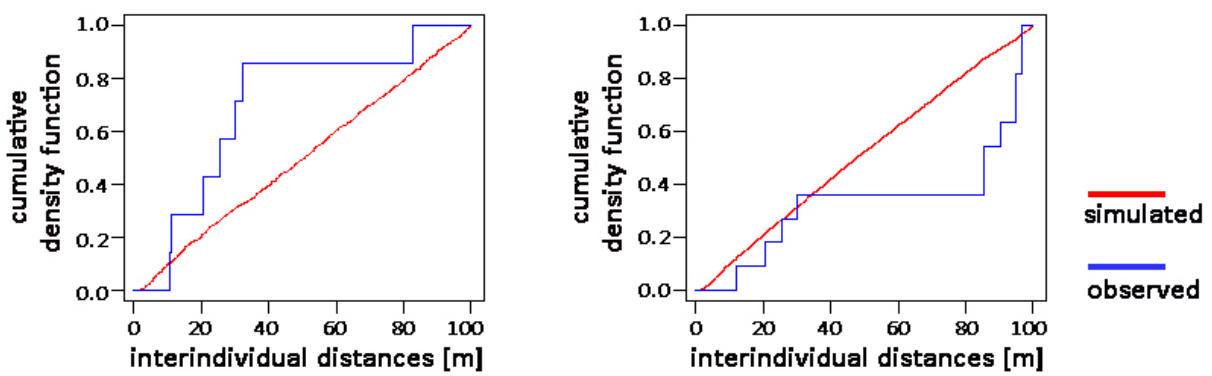

Fig. 70: Microcebus murinus - C. medius interspecific pairs (rainy season) on section NN 7 with stronger, and on section Kir W with less, spatial association than expected by chance

The remaining three sections did not indicate interspecific associations to differ from a random spatial distribution $(C N 2.1: p=0.479, n=11 ; C N$ 3.2: $p=0.435, n=20 ; A 06 p=0.721$, $\mathrm{n}=4$ ). Figure 71 graphically depicts interindividual distance distribution on only those

CN 2.1
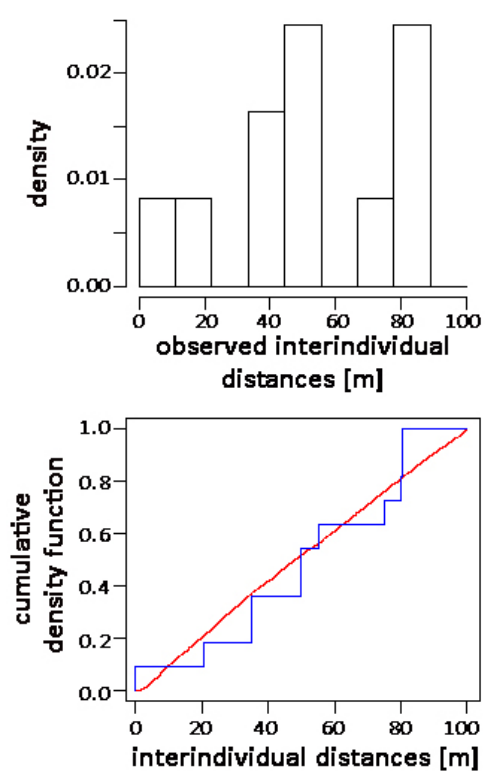

CN 3.2
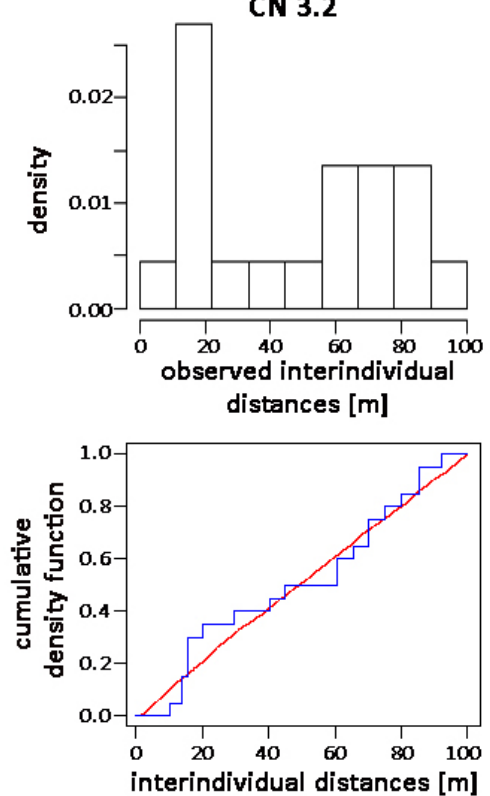

sections with high sample sizes

(section $\quad A \quad 06$ omitted).

Fig. 71: Microcebus murinus interspecific pairs with C. medius (rainy season) on sections CN 3.2 and CN 2.1, on which individuals were intraspecifically associated in space as expected by chance 
Comparing the proportion of individuals $C$. medius in close association with either of the mouse lemurs, we found significantly more individuals than expected in close vicinity to $M$. berthae, and simultaneously a smaller number of pairs within $100 \mathrm{~m}$ were realized with $M$. murinus $\left(\chi^{2}=61.842, d f=1, p=0.000\right)$. These results reveal small-scale spatial avoidance indicative for intense interspecific competition between $M$. murinus and $C$. medius.

\section{Interspecific distribution of individuals within the trapping web}

In contrast to the local distribution of individuals within sections, our trapping web captures clearly documented small-scale spatial separation of $M$. berthae and $M$. murinus individuals in non-degraded grid system N5 (figure 72). The gradual increase in the number of trapped M. murinus individuals, with simultaneously decrease in $M$. berthae over subsequent trapping web session is most likely to be attributed to divergent seasonal trapability in either species. The probability to capture $M$. berthae individuals is considerably lower during the rainy than during the dry season, whereas seasonal variation in trapping success is less pronounced in M. murinus.
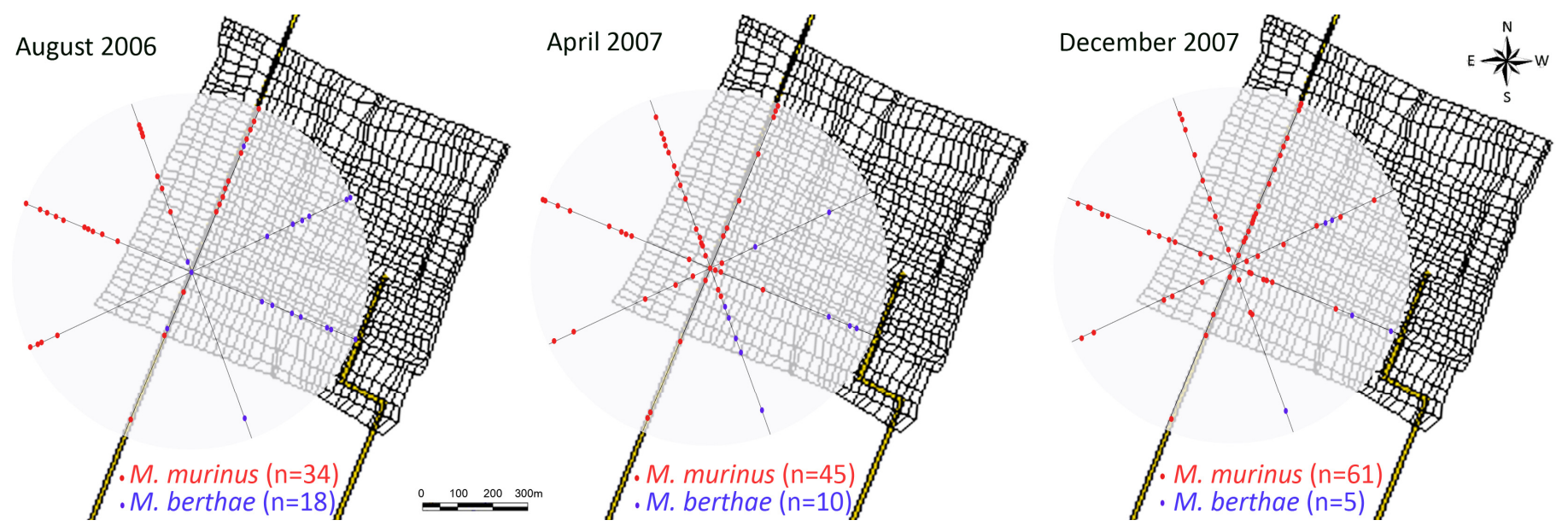

Fig. 72: Microcebus spp.'s first captures within the trapping web

On the majority of trap locations, we did not capture any Microcebus individual. Where we captured Microcebus spp., trap locations were predominately occupied by one of the species. We observed both species on the same trap location within the same session in only two cases, both during peak rainy season 2007 (figure 73), which might be ascribed to released competitive pressure, but could also be a consequence of behavioral changes in the breeding season. 


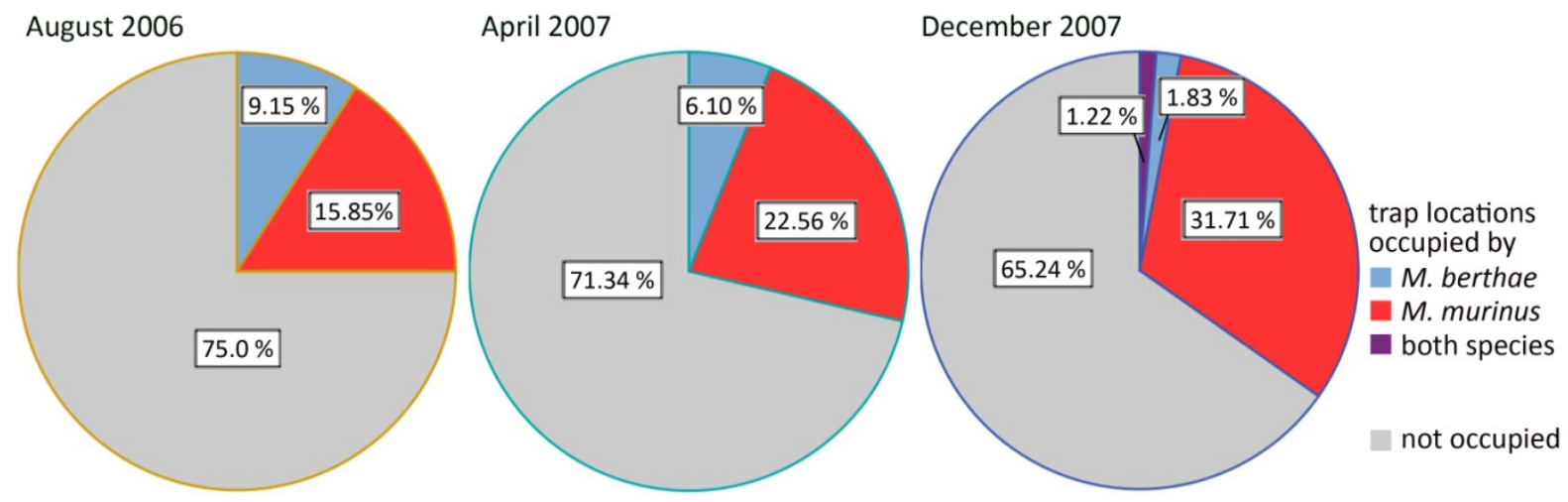

Fig. 73: Occupation of trap locations within the trapping web by Microcebus' spp. (first captures)

Co-occurrence analysis in EcoSim clearly confirmed interspecific checkerboard distributions in all trapping web sessions. The average number of checkerboard units significantly exceeded the simulated C-score values in all capture sessions (table 12).

Table 12: C-scores observed and expected based on 5000 simulations (mean \pm SD)

\begin{tabular}{|c|c|c|c|c|}
\hline year & month & C-score $_{\text {obs }}$ & C-score $_{\text {exp }}$ & p (obs > exp) \\
\hline 2006 & August & 390.00 & $97.41 \pm 1200.20$ & $<0.001$ \\
\hline 2007 & April & 370.00 & $93.72 \pm 1882.18$ & $<0.001$ \\
\hline 2007 & December & 156.00 & $54.56 \pm 2101.32$ & 0.032 \\
$*$
\end{tabular}$*$

\section{DISCUSSION}

\section{Habitat classification}

Our ground-based classification of transects into non-degraded and degraded habitat matched the forest classification by Zinner \& Torkler (2005; figure 3) and therefore appears reliable. Non-degraded habitat (roughly corresponding to forest classes 1 and 2) distinguished from degraded habitat (forest classes 3 to 6 ) in a higher and more closed canopy. Canopy cover was associated with higher tree density in mature trees, as well as in trees that older than 10 years.

\section{Cheirogaleid population structure}

\section{Microcebus berthae}

The regional distribution of $M$. berthae's across forest regions and across transects reflected high ecological specialization and confirmed the locally endemic species' sensitivity to anthropogenic disturbances. The regional distribution of Microcebus berthae complies with 
the GIS-based allocation of anthropogenic pressure by Smith et al. (1997) in that the species was virtually absent from RSA and west of the Route National. The authors proposed human impact on the forest to be most severe in the south-western part of Menabe Central, where the Route National traverses the forest and inhabitants of several villages (Marofandilia, Kirindy Village, Beroboka, Lambokely) harvest the forest and its products for subsistence or clear it for cultivation (Réau 2002). Relatively low forest accessibility in Ambadira by trafficable roads and a comparably sparse human population may explain that $M$. berthae occurs in highest population densities in the northernmost forest part despite lack of effective protection. An overall density estimate of 80 individuals per $\mathrm{km}^{2}$ for Menabe Central is likely reliable, but estimates for particular forest parts should be regarded with caution as they represent unweighted means of survey-specific results.

Complying with other vertebrate specialists that have narrow ecological niches (Swihart et al. 2003, Swihart et al. 2006), Microcebus berthae's population densities were highest in most suitable core areas in greater distance from its range boundary. Only a fraction of transects were colonized and the population was largely restricted to nondegraded habitat. During the rainy season, a majority of the population concentrated in nondegraded habitat, where increases in carrying capacity are presumably most pronounced. In addition, intact habitat provides preferable structural characteristics that are of particular significance for breeding in the rainy season (liana and a high herb layer cover to build liana or leaf nests: Rendigs et al. 2003). During the dry season, M. berthae's population spread out to degraded habitat, which is in accordance with the compensation of resource scarcity by feeding on secretions of homopteran larvae (Dammhahn \& Kappeler 2008, 2010) that aggregate along forest edges (Corbin \& Schmid 1995). Log-linear models reconfirmed seasonal differences in factors determining $M$. berthae's regional distribution. The vicinity of villages negatively affected population structure only during the dry season, whereas it did not have a significant influence $M$. berthae's distribution during the rainy season, when forest accessibility is reduced. This corresponds to Smith et al. (1997), who proposed anthropogenic disturbances to intensify when the forest is accessible (e.g. for that harvesting of forest products). Therefore, $M$. berthae may indeed track differential seasonal changes in carrying capacity as well as in human frequentation across heterogeneous habitats. 
Our captures vaguely supported $M$. berthae individuals to be rather confined to intact habitat, but results were not conclusive. As trapping success in cheirogaleids was found to increase with decreasing productivity (Lahann et al. 2006), lower population sizes might have been compensated by enhanced trapability in degraded habitats where resources are scarce.

\section{Microcebus murinus}

The hypothesized resilience against anthropogenic disturbances in widely distributed generalist $M$. murinus was supported on the population level by distance samples and captures. The gray mouse lemur was present on the vast majority of transects in an overall density of 182 individuals per $\mathrm{km}^{2}$. Population distribution was heterogeneous across forest regions, with lowest densities in the northernmost forest part (Ambadira), and highest in RSA.

Gray mouse lemur populations were heterogeneously distributed also across transects. We did not find unambiguous support for seasonal population shifts between habitats of different quality, but a characteristic pattern in the distribution of $M$. murinus density classes across degraded and non-degraded habitat transects might yet indicate capacity tracking. We encountered $M$. murinus on all degraded habitat transects during the productive rainy season, but not during the dry season. In either season, the species was absent from some transects in non-degraded habitat. Only during the rainy season, Gray mouse lemurs occurred in high population densities on some non-degraded habitat transects. Therefore, M. murinus populations may spread out by local dispersal (cf. Snyder \& Chesson 2003) during the rainy season across degraded habitats, parts of which might not be suitable during the dry season. In addition, the species may track pronounced capacity changes in non-degraded habitat during the rainy season, which then supported higher population densities. Lack of seasonal differences in overall encounter rates challenge the alternative explanation that the pattern is simply caused by dry season inactivity in most females. Moreover, log-linear models of $M$. murinus' regional distribution reconfirmed seasonal differences in population structure as spatial affiliation with villages was confined to the dry season.

Our captures also supported that $M$. murinus is widely unimpaired by human activities and resistant to habitat alteration (Ganzhorn et al. 2003). However, we did not find 
females' capacity to enter energy-saving torpor hampered in degraded habitat during the dry season (Ganzhorn \& Schmid 1998). A shortcoming of our analysis is that we only compared sex-specific capture rates, but did not test the body condition of captured individuals for differences potentially reflecting poor suitability of degraded habitat to $M$. murinus.

\section{Interspecific interactions}

\section{Direct interspecific interactions}

\section{Ecological similarity of $\mathrm{M}$. berthae and $\mathrm{M}$. murinus}

Our analysis of interindividual distributions within section supported interspecific differences in competitive strategies. In accordance with earlier results of a small-scale study (Dammhahn \& Kappeler 2005, 2008, 2009), we found intraspecific clumping of individuals less pronounced in $M$. berthae than in $M$. murinus. The wide intraspecific distribution of $M$. berthae individuals corresponds to the reliance on dispersed food resources, which leads to intense scramble competition. We observed more than one $M$. berthae individual on a greater number of sections surveyed during the rainy than during the dry season, but the sections with short-distance pairs was fewer during the rainy season. The more clustered distribution of $M$. murinus complies with intraspecific contest competition for clumped food resources. Like in $M$. berthae, sections with more than one individual were more numerous during the rainy than during dry season, but sections harboring short distance pairs were fewer. The observed association of individuals can therefore not simply be explained by mother-infant associations during the rainy season, which would be expected to stay in close vicinity of each other. Modeling of interindividual distributions within sections yielded ambiguous results in both species and did not support determinative intraspecific associations of Microcebus individuals on the local scale.

\section{Interspecific competition in Microcebus spp.}

There are clear indications for spatial separation of the two mouse lemur species on the regional scale. The sister species' regional distribution across forest regions was in parts negative complementary, a biogeographic pattern that may have arisen from interspecific competition. 
Microcebus spp. distribution across transects indicated that interspecific competition was indeed most intense during the dry season and in degraded habitat. In addition, the gray mouse lemur was attracted to villages during the dry season, whereas $M$. berthae evaded anthropogenic environments. Anthropogenic environments might provide additional dry season resources to the generalist and also homopteran larvae on plenty of forest edges (Corbin \& Schmid 1995). Population-level affiliation of M. murinus with villages would lower interspecific competition in more distantly located degraded habitat. By this means, $M$. berthae could spread to those parts of degraded habitat providing them with essential homopteran secretions along edges, but are not heavily exploited by villagers. Alternatively, M. murinus could be excluded from forest edges in greater distance from village by interspecific competition with other cheirogaleids, and crowded into anthropogenic environments that are not suitable to coexisting species. In either case, habitat heterogeneity facilitates coexistence of Microcebus spp. by a spatial storage effect: the two species are relieved from intense interspecific competition in different habitat types, and a concentration into the respective refuges by local dispersal prevents competitive exclusion (Chesson 2000b). Conspicuously, we observed an aggregation of $M$. berthae individuals during the rainy season only on the one transect on which we did not detect a single $M$. murinus individual over the entire study period (A 04 in Ambadira forest).

However, log-linear models did not reveal negative interspecific associations of Microcebus populations on the regional scale. Our models left a great proportion of the total variation in measures of abundance unexplained, which may be due to a variety of factors (Schweiger et al. 1999). A key limitation of species-oriented approaches is that not all important variables can be included in the analysis. Additional variables, such as resource distribution or interactions with additional species (e.g. members of the food web) can have a major impact on the stability of communities (e.g. Holt \& Polis 1997). We did not consider floristic diversity in our analysis, which was shown to be more important than structural diversity for lemurs, as they require a certain number of tree species that guarantee food supply despite pronounced seasonality (Ganzhorn 1999). Neither did we incorporate the distribution of homopteran insect larvae, which provide cheirogaleids with essential dry season resources (Corbin \& Schmid 1995, Dammhahn et al. 2009, 2010).

Analyses of interindividual distributions did not yield unambiguous results. Microcebus berthae individuals co-occurred with $M$. murinus only on a minor fraction of 
sections sampled during the dry as well as during the rainy season. Interspecific associations of $M$. berthae and $M$. murinus individuals did not vary with resource supply in the predicted way, as we found the two species in close association on a higher proportion of transects during the dry than during the rainy season. Interspecific competition between Microcebus individuals within sections did not appear intense and did not exceed intraspecific competition. Spatial exclusion on the individual level does therefore appear unlikely based upon the local scale analysis of interindividual distribution. In contrast, interspecific spatial separation of Microcebus individuals was clearly documented by the population examined by trapping web captures, which has been approved to be stable over several years in extensive capture-mark-recapture studies (Dammhahn \& Kappeler 2008, 2009, 2010).

The obvious disparity between our local scale observations and trapping web captures may be ascribed to the sampling methods, which may be insufficient to measure competition. Microcebus spp. compete interspecifically rather by depleting resources than by monopolizing them, as emphasized by increased home range sizes in areas of interspecific coexistence (Dammhahn \& Kappeler 2009). If the superior competitor displaces the inferior one by resource suppression rather than by physical exclusion from the habitat, interspecific competition might not become apparent in the distribution of individuals (Hardin 1960, Holt et al. 1994). According to Hastings (1987), the dynamic processes of competition are not detectable based on static observations of species distributions and abundances even under idealized conditions. Moreover, intense interspecific interactions in the past may still determine the behavior, distribution or morphology of species. The intensity of interactions, however, may have diminished over evolutionary times by adaptive responses that facilitate coexistence (Holt \& Polis 1997), so that present day interactions are hard to measure (Connell 1980, Holt 1984).

However, comprehensive examinations of feeding niches and microhabitat utilization in Microcebus spp. indicated that differentiation is insufficient to prevent interspecific exclusion. Socio-ecological aggregation patterns in $M$. murinus have been considered to predominately stabilize mouse lemur coexistence by locally increasing the intensity of intraspecific relative to interspecific competition (Dammhahn \& Kappeler 2008, 2009, 2010, but see Schwab \& Ganzhorn 2004). 


\section{Indirect interspecific interactions}

Our study shows that interspecific interactions with third agents additionally stabilize coexistence of Microcebus spp. by structuring the mouse lemur populations. Interspecific distribution patterns on various scales fulfilled our predictions for a third agent's stabilizing impact on the coexistence of Microcebus spp. Two cheirogaleid species, $M$. coquereli and $C$. medius, appear to jointly stabilize interspecific coexistence in mouse lemurs by means of interspecific interactions.

\section{Population level}

We identified $M$. coquereli as the key agent to stabilize interspecific coexistence of Microcebus spp. on the population level, most likely via intraguild predation. With the exception of RSA, population densities of $M$. murinus and $M$. coquereli were negative complementary across forest regions, whereas $M$. berthae's global distribution matched the regional distribution of $M$. coquereli. Log-linear models of the interspecific distribution across transects suggested that in either season, $M$. coquereli regulates $M$. murinus population densities particularly in non-degraded habitat, which $M$. berthae relies on. Predation represents a plausible determinant of ecological structure as its impact on the structure of species assemblages often outweighs that of resource competition (Paine 1966, Holt 1984). Predation most likely facilitates interspecific coexistence of prey species at intermediate levels of productivity (Holt \& Polis 1997, Bonsall \& Holt 2003). Opportunistic predation on $M$. murinus in non-degraded habitat under scarce resource supply is therefore intuitive during the dry season (cf. Schliehe-Diecks et al. 2010). However, during the rainy season, $M$. coquereli's regulative impact on $M$. murinus population may rather by attributed to intensified feeding competition in preferable habitat among capacity tracking cheirogaleids. Habitat selecting predators often stabilize interspecific coexistence by forcing prey into certain habitat types (Holt 1984), if landscape heterogeneity provides species with refuges from predation (Holt \& Polis 1997). In our model system, interspecific interactions with $M$. coquereli enhanced spatial heterogeneity in Menabe Central and by this means stabilize the coexistence of the two mouse lemur species. This represents an agent-mediated spatial storage effect in non-degraded habitat, where Mirza down-regulates the population of $M$. murinus and provides $M$. berthae with refuges from competition (cf. Chesson 2000b, Snyder \& Chesson 2003). In contrast, M. murinus appears to profit from anthropogenic 
environments that are of little suitability to other cheirogaleids. Gray mouse lemurs might escape negative interspecific interactions with the third agent by crowding in the vicinity of villages. In this scenario, the observed negative complementary distribution of Microcebus populations in relation to the distance from villages would be a consequence of indirect interactions (apparent competition: e.g. Holt \& Lawton 1994). Alternatively, lack of the third agent's determinative impact on $M$. murinus' population structure in anthropogenic environments might locally release the species from predation pressure and favor direct interspecific exclusion of $M$. berthae.

C. medius could be excluded as a third competitor that shapes the ecological structure of Microcebus populations in Menabe Central. Overlapping ecological requirements of $M$. murinus and $C$. medius might prevent interspecific negative associations on a large spatial scale, while $M$. berthae may not be positively associated with $C$. medius due to differential microhabitat preferences (Schwab \& Ganzhorn 2004). Interspecific exclusion might be prevented due to $M$. murinus' acceptance of habitats that are not suitable to $C$. medius and therefore represent competitor-free refuges. We cannot judge the situation during the dry season when $C$. medius hibernates, but the species presumably affects $M$. murinus' population to some extent by reducing the number of available sleeping holes.

\section{Individual level}

Interspecific coexistence in Microcebus spp. appears to be regulated by the third agents additionally on the individual level. Interaction regimes that release $M$. berthae from intense competition with the congener change with the seasons. During the dry season, spatial avoidance of $M$. coquereli individuals by $M$. murinus was more pronounced than by $M$. berthae and $M$. coquereli rather associated with $M$. berthae than with $M$. murinus. This pattern is likely a consequence of intensified intraguild predation when resources are scarce. Resource competition likely affect both mouse lemurs, explaining the lack of positive associations of $M$. coquereli and $M$. berthae individuals. Opportunistic predation, however, disproportionally affects $M$. murinus and likely accounts for the observed negative spatial associations of individuals. According to the interspecific distribution of individuals, $M$. coquereli stabilizes interspecific coexistence of Microcebus spp. also on the local scale. 
During the rainy season, the interspecific distribution of individuals strongly indicated the importance of $C$. medius for the regulation of mouse lemur competition. Microcebus murinus avoided the vicinity of $C$. medius individuals, whereas $M$. berthae and $C$. medius individuals were found in close spatial association. Microcebus berthae therefore appears to profit on a local scale from competitor-free space created by $C$. medius via interspecific competition with M. murinus (Schwab \& Ganzhorn 2004). The impact of $C$. medius is presumably most pronounced in non-degraded habitat and far from villages, where the species occurs in highest densities. This means that also $C$. medius regulates mouse lemur competition in the habitat type essential to M. berthae.

Notably, the complementary association pattern of either third agent with Microcebus spp. on a regional scale was opposed to the interspecific distribution pattern of the mouse lemurs. On the largest scale, $M$. berthae only occurs were either $M$. coquereli population densities are high (Ambadira) or those of $C$. medius (Kirindy, Corridor). The forest part, on which both third agents occurred in rather low densities (RSA) did not support $M$. berthae populations. Moreover, interspecific spatial exclusion in Microcebus spp. across transects were most pronounced during the dry season in degraded habitat, where interspecific interactions with $M$. coquereli did not regulate interspecific competition. These patterns corroborate our hypothesis that interactions with third agents stabilize interspecific coexistence of Microcebus spp. on a regional scale.

\section{System stability in view of habitat change}

\section{Community composition across heterogeneous habitat}

In consequence of differential susceptibilities of member species to habitat degradation and anthropogenic activities, the cheirogaleid assemblage in Menabe Central varied in composition across heterogeneous habitats. Low overall population densities in M. coquereli correspond to results of earlier studies (e.g. Hladik et al. 1980). Even though the species appeared to be fairly resistant to human impact according to our data, $M$. coquereli's distribution has long been recognized to be related to local changes in forest structure and composition (Petter et al. 1971, in Hladik et al. 1980). Cheirogaleus medius also occurred in comparably high population densities and was homogeneously distributed across forest regions. The overall population density estimate of $180 \mathrm{ind} . / \mathrm{km}^{2}$ in Kirindy forest (table 6) slightly deceeds the density range of 218 ind./ km $^{2}$ given by Fietz (2003). During rainy season 
activity, C. medius relied on non-degraded habitat and avoided the vicinity of villages, which corresponds to the negative edge response by congener $C$. major in south-eastern Madagascar (Lehmann et al. 2006b). Lowest over-all density and a fairly homogeneous distribution across Menabe Central were found in P. pallescens (table 6). A density estimate of 32 ind./ $\mathrm{km}^{2}$ for Kirindy Forest considerably deceeds previous estimates of $153-555$ ind./ $\mathrm{km}^{2}$ that were also based on line transect sampling (Ganzhorn \& Kappeler 1996). Encounter rates did not differ between degraded and non-degraded habitat, but the fork-marked lemur evaded the proximity of villages. Corresponding to Hladik et al. (1980), we did not document seasonal variation in encounter rates.

The observed pattern is in accordance with earlier studies, which found $\alpha$-diversity in lemurs highest at medium disturbance levels (Ganzhorn 1999) and dropping with increasing agricultural intensity (Lehman et al. 2006a). Our cheirogaleid assemblage was deprived of one or more species where anthropogenic pressure is particularly intense (west of the Route National and around Marofandilia and Kirindy Village: figures 16 and 75). Forest accessibility and intensity of exploitation plausibly explained observed variation in lemur community composition. Our results therefore reconfirm those of a rapid fauna survey in Menabe Central that suggested lemur community composition to be a function of human activity (Smith et al. 1997). The authors identified cultural variables, primarily slash and burn agriculture, root harvesting and stock grazing in village environments, as better predictors of lemur species richness and abundance than natural site variation. Consistently, we observed even those species that are susceptible to anthropogenic disturbances in the northernmost forest part Ambadira, which is rather moderately accessed by roads and only marginally populated, but has not been effectively protected. In contrast, those species are largely absent from the Réserve Spécial d'Andranomena that was supposed to be protected for several decades, but in great parts comprises of degraded and situated in vicinity of two greater villages (Marofandilia and Kirindy Village). We therefore agree with Smith et al. (1997), that this government nature reserve is not the appropriate location for lemur conservation in Menabe Central. Further conservation efforts should rather be directed to Ambadira forest, and therein to the southernmost part that borders on the corridor. In consequence of low accessibility, we could not survey this particular area, but for the same reason the region seems most promising for biodiversity conservation. 
Species in fragmented habitat experience consequences of spatial subdivision differentially (Fischer \& Lindenmayer 2007). Lemur diversity was found to decline with decreasing fragment size and species in fragments were deterministically driven to extinction, i.e. in predictable sequence (cf. Ganzhorn 1999, Ganzhorn et al. 1999, Ganzhorn \& Eisenbeiß 2001, Irwin \& Raharison 2009, Irwin et al. 2010). Of our model species, M. berthae is most sensitive to fragmentation: only fragments exceeding 30,000 hectares support viable populations in Menabe Central. On the contrary, M. murinus even occupies tiny patches of no more than one hectare. Also C. medius relies on larger habitat patches of more than 600 hectares in Menabe Central, whereas $M$. coquereli was observed in relatively small fragments of only six hectares (Ganzhorn et al. 2003).

\section{Indirect effects resulting from habitat loss and fragmentation}

Differential susceptibilities of cheirogaleids to habitat degradation and fragmentation have implications for interspecific interactions that stabilize the coexistence of the mouse lemurs. Present-day composition of the cheirogaleid assemblage in Menabe Central essentially depends on the continuity of interspecific interactions with third agents that stabilize Microcebus spp.'s interspecific coexistence. Loss of a single species, even if originally rare, may trigger an extinction cascade that potentially extends to a large number of species and therefore have dramatic consequences for community stability (Allesina \& Levine's 2011). If one of the species stabilizing the system of coexisting cheirogaleids was driven to extinction, the ecological structure of the assemblage could essentially be changed in a way threatening M. berthae's persistence.

On the individual level, interspecific competition between Microcebus spp. would likely intensify in absence of $C$. medius. However, the stability of mouse lemur coexistence most importantly depends on $M$. coquereli's regulative impact. Mirza represents the keystone species for cheirogaleid community structure in Menabe Central, as it prevents competitive exclusion during the dry season, in which minimum resource supply determines overall carrying capacity of the habitat (Hladik et al. 1980), and acts on both, the individual and the population level. In absence of the intraguild predator, M. murinus populations would likely increase and might competitively exclude $M$. berthae from non-degraded habitat it relies on. Interspecific coexistence that is stabilized by predation substantially depends on movements between habitat patches by the prey species as well as by the 
predator (Holt 1984). Even though observed in comparably small forest fragments (Ganzhorn et al. 2003), $M$. coquereli populations are subject to considerable fluctuations in size that are compensated by high migration rates (Markolf et al. 2008). Thus, continuing fragmentation may put $M$. coquereli at risk of extinctions from fragments that are too remote to allow for recolonization. In consideration of proceeding fragmentation of western dry forests, the IUCN rated $M$. coquereli as "near threatened" despite its comparably wide biogeographic distribution (Andrainarivo et al. 2008, in IUCN 2010).

\section{Recommendations for conservation}

As a highly charismatic species at risk of global extinction, Mme. Berthe's mouse lemur qualifies as a flagship species to preserve the largest remnant of dry deciduous forest in western Madagascar and the exceptional biodiversity found in this Global 200 ecoregion (Dinerstein et al. 1996). Our data support the IUCN classification of $M$. berthae as "Endangered" (EN), as it meets several of the criteria A to $E$ indicating a high risk of extinction in the wild.

Microcebus berthae's geographic range is limited to $650 \mathrm{~km}^{2}$ forested area in Menabe Central, and our study shows that $M$. berthae effectively occupies only about half of the area and no more than five locations. Combined with a continuing decline (iii) in habitat extent and quality as well as (iv) in the number of locations and subpopulations (in RSA and west of $\mathrm{RN}), M$. berthae's global range meets the IUCN range size criteria B1a and B1b as well as the area of occupancy criteria B2a and B2b for category EN. Confinement to no more than five areas does also meet population size criterion D2 for "Vulnerable" (VU), despite a comparably high population density estimate of 80 individuals per $\mathrm{km}^{2}$ across Menabe Central. In order to warrant $M$. berthae's persistence, it is imperative to prevent further habitat loss, fragmentation and degradation in Menabe Central.

Continuing anthropogenic disturbances in Menabe Central will place the cheirogaleid assemblage in jeopardy of substantial deprivation. We cannot judge population trends over the past 10 years (IUCN criteria $A 1$ and $A 2$ ), as long-term data on specific distributions and abundances are deficient. However, population declines in all species is to be expected due to $(A 3 c)$ continuous decline in extent and quality of suitable habitat and $(A 3 d)$ increasing exploitation, which will further impinge on the species' extent of occurrence and area of occupancy. 
In consequence of $M$. berthae's reliance on complex interspecific interaction regimes, its persistence depends on co-occurring key stone species $M$. coquereli and $C$. medius and on competitor M. murinus (A3e). Those species need to be included into continuous population monitoring across $M$. berthae's biogeographic range in order to detect disruptions in dynamic processes that essentially stabilize the cheirogaleid assemblage. Comprehension of local dispersal rates and distances in heterogeneous habitat are essential to understand the stability of interspecific coexistence based on an agent-mediated spatial storage effect (Chesson 2000b, Snyder \& Chesson 2003, Amarasekare et al. 2004). In addition, we need to understand if the fragmented habitat context subdivides populations or if movements between patches are sufficient to support a functional metapopulation (Hanski \& Simberloff 1997). The equilibrium state of metapopulations needs to be continuously surveyed, particularly if anthropogenic disturbances progress (Howe \& Davies 1991). Colonizationextinction dynamics may warrant regional persistence of predators and their prey, even though local interactions are unstable (Holt \& Polis 1997). Conversely, fragmentation may act synergistically with predation if spatial isolation prevents recolonization by prey after extirpation from a patch (Irwin et al. 2009).

Persistence of $M$. berthae additionally depends on the conservation of non-degraded habitat content. In tropical dry forests, succession after abandonment results in vegetation that comprises a poor subset of the original flora. Such secondary dry forests will persist and characterize the site for several hundred years. Predominately based on wind-dispersal, habitat content is relatively inhospitable to animals, poor in terms of biodiversity and more deciduous than forests that have succeeded through vertebrate seed-dispersal (Janzen 1988). The dry deciduous forest in Menabe Central is particularly vulnerable to human incursions, as the inherently arid climate does has not been favoring the natural regeneration of endemic wood taxa (Hunziker 1981, in Réau 2002). Forest regeneration will be further restrained in the future as aridity aggravates in consequence of climate change (Hannah et al. 2008).

Changes in habitat content and context may take considerable time to manifest in the structure of communities (Fischer \& Lindenmayer 2007). Isolated populations may persist for considerably time as "living dead" in unsuitable habitats even though being ultimately doomed (e.g. Turner \& Corlett 1996, Gibbons \& Harcourt 2009), or be sustained merely by directional immigration (Amarasekare et al. 2004). Therefore, viable populations 
need to be distinguished from remnant or sink populations (Irwin et al. 2010), and population viability analyses are required to estimate $M$. berthae's probability of extinction (IUCN criterion E). The great variety of dynamic processes stabilizing the present-day cheirogaleid assemblage can only be comprehensively examined by integrating population genetic studies (cf. Markolf et al. 2008, Olivieri et al. 2008).

Immediate and sound conservation practices are imperative if we are to protect the exceptional biodiversity in Menabe Central. Species collapses in Madagascar have been documented even in relatively large forest stands of more than 10,000 hectares, where fragmentation and forest edges are not yet obvious (Ganzhorn et al. 2001), and species once lost never reappeared in impoverished successive communities (Atmar \& Patterson 1993, Ganzhorn et al. 2003). In Menabe Central, another locally endemic flagship species, the Giant Jumping Rat (Hypogeomys antimena), has already been driven to the brink of extinction in consequence of habitat reduction and fragmentation combined with indirect effects of human frequentation (Sommer et al. 2002). Ganzhorn et al. (2001) considered it unlikely that biodiversity in Western Madagascar can be preserved in the long run, as forest cover has been continuously reduced and fragmented for decades. Complete isolation of Menabe Central from other large dry forest fragments will prevent the great majority of species from shifting their ranges in response to climate change (Soulé et al. 2004).

\section{ACKNOWLEDGEMENTS}

My project was coordinated at the German Primate Center, Dpt. of Behavioral Ecology \& Sociobiology/ Anthropology, and in the PhD program of the Göttingen Center of Biodiversity and Ecology of the Georg-August University of Göttingen. I owe special thanks to my advisor Peter Kappeler for many years of excellent training and broad support in my academic career and to Hans-Rolf Gregorius, Joachim Saborowski, and Walter Zucchini for many hours of fruitful discussions on my project and for statistical advice. The manuscript benefited from these persons' helpful comments and from the pictures and maps provided by Manfred Eberle and Dietmar Zinner. My work was completed with financial support from the German Primate Center (DPZ), Primate Conservation Inc. (PCI), Margot Marsh Biodiversity Foundation (Conservation International), and Durrell Wildlife Conservation Thrust (DWTC) and with authorization by the CAFF/CORE committee and the National Association for the Management of Protected Areas (ANGAP). I thank Melanie Dammhahn for introducing me to animal handling and Léon Razafindrakoto and Rodin Rasoloarison for logistic support as well as for helpful suggestions during field work. Most importantly, I am deeply indebted to the members of my high performance team Nielsen Rabarijaona, Remy Ampataka, Raza Rakotonirina, Jean-Pierre Tolonjanahary, and Tiana Andrianjanahary for their expert assistance and for their outstanding commitment to our surveys. I also yield thanks to my students, particularly to Zo Elia Mevanarivo from the Département des Eaux et Forêts of the University of Antananarivo for the invaluable collaboration. Last but not least I thank all the Malagasy staff in Kirindy Forest for their great amicability and a magnificent intercultural experience. 


\section{REFERENCES}

Allesina, S., and J. M. Levine. 2011. A competitive network theory of species diversity. PNAS 108:5638-5642.

Amarasekare, P. 2003. Competitive coexistence in spatially structured environments: a synthesis. Ecology Letters 6:1109-1122.

Amarasekare, P., M. F. Hoopes, N. Mouquet, and M. Holyoak. 2004. Mechanisms of Coexistence in Competitive Metacommunities. The American Naturalist 164:310-326.

Amarasekare, P., and R. M. Nisbet. 2001. Spatial Heterogeneity, Source-Sink Dynamics, and the Local Coexistence of Competing Species. American Naturalist 158:572-584.

Andrainarivo, C., V. N. Andriaholinirina, A. Feistner, T. Felix, J. Ganzhorn, N. Garbutt, C. Golden, B. Konstant, E. Louis Jr., D. Meyers, R. A. Mittermeier, A. Perieras, F. Princee, J. C. Rabarivola, B. Rakotosamimanana, H. Rasamimanana, J. Ratsimbazafy, G. Raveloarinoro, A. Razafimanantsoa, Y. Rumpler, C. Schwitzer, U. Thalmann, L. Wilmé, and P. C. Wright. 2008. IUCN Red List of Threatened Species. 2010. IUCN.

Antón, M., A. Galobart, and A. Turner. 2005. Co-existence of similar-toothed cats, lions and hominins in the European Pleistocene. Implications of the post-cranial anatomy of Homotherium latidens (Owen) for comparative palaeoecology. Quaternary Science Reviews 24:1287-1301.

Atmar, W., and B. D. Patterson. 1993. The measure of order and disorder in the distribution of species in fragmented habitat. Oecologia (Berlin) 96:373-382.

Aunapuu, M., and T. Oksanen. 2003. Habitat selection of coexisting competitors: a study of small mustelids in northern Norway. Evolutionary Ecology 17:371-392.

Basset, Y., O. Missa, A. Alonso, S. E. Miller, G. Curletti, M. Meyer, C. Eardley, O. T. Lewis, M. W. Mansell, V. Novotny, and W. T. 2008. Choice of metrics for studying arthropod responses to habitat disturbance: one example from Gabon. Insect Conservation and Diversity 1:55-66.

Beisner, B. E., D. T. Haydon, and K. Cuddington. 2003. Alternative Stable States in Ecology. Frontiers in Ecology and the Environment 1:376-382.

Bentley, J. M., C. P. Catterall, and G. C. Smith. 2000. Effects of Fragmentation of Araucarian Vine Forest on Small Mammal Communities. Conservation Biology 2000:1075-1087.

Bihn, J. H., M. Verhaagh, M. Brändle, and R. Brandl. 2008. Do secondary forests act as refuges for old growth forest animals? Recovery of ant diversity in the Atlantic forest fo Brazil. Biological Conservation 141:733-743.

Billick, I., and T. J. Case. 1994. Higher order interactions in ecological communities: What are they and how can they be detected? Ecology 75.

Bisseleua, D. H. B., A. D. Missop, and S. Vidal. 2009. Biodiversity Conservation, Ecosystem Functioning, and Economic Incentives under Cocoa Agroforestry Intensification. Conservation Biology 23:1176-1184.

Bonsall, M. B., and M. P. Hassell. 1997. Apparent competition structures ecological assemblages. Nature 388:371-373.

Bonsall, M. B., and R. D. Holt. 2003. The Effects of Enrichment on the Dynamics of Apparent Competitive Interactions in Stage-Structured Systems. The American Naturalist 162:780-795.

Bowers, M. A., and J. H. Brown. 1982. Body size and coexistence in desert rodents: chance or community structure? Ecology 63:391-400.

Braune, P., S. Polenz, V. Zietemann, and E. Zimmermann. 2001. Species-specific signaling in two sympatrically living nocturnal primates, the grey and the golden-brown mouse lemur (Microcebus murinus and Microcebus ravelobensis), in Northwestern Madagascar. Page 126 in R. Apfelbach, M. Fendt, S. Krämer, and B. M. Siemers, editors. XXVII International Ethological Conference. Advances in Ethology, Tübingen, Germany.

Braune, P., S. Schmidt, and E. Zimmermann. 2005. Spacing and group coordination in a nocturnal primate, the golden brown mouse lemur (Microcebus ravelobensis): the role of olfactory and acoustic signals. Behav Ecol Sociobiol 58:587-596.

Brower, J. E., J. H. Zar, and C. N. Von Ende 1990. Field and laboratory methods for general ecology. WCB/ McGraw-Hill, Dubuque, lowa.

Brown, J. H. 1973. Species Diversity of Seed-Eating Desert Rodents in Sand Dune Habitats. Ecology 54:775-787.

Brown, J. H. 1984. On the relationship between abundance and distribution of species. American Naturalist 124:255-279.

Brown, J. H., and G. A. Lieberman. 1973. Resource Utilization and Coexistence of Seed-Eating Desert Rodents in Sand Dune Habitats. Ecology 54:788-797.

Brown, J. H., D. W. Mehlman, and G. C. Stevens. 1995. Spatial Varation In Abundance. Ecology 76:2028-2043.

Brown, J. H., G. C. Stevens, and D. M. Kaufman. 1996. The Geographic Range: Size, Shape, Boundaries, and Internal Structure. Annual Review of Ecological Systems 27:597-623. 
Brown, K. A., and J. Gurevitch. 2004. Long-term impacts of logging on forest diversity in Madagascar. PNAS 101:6045-6049.

Brown, K. M. 1982. Resource Overlap and Competition in Pond Snails: An Experimental Analysis. Ecology 63:412-422.

Buckland, S. T., D. R. Anderson, K. P. Burnham, J. L. Laake, D. L. Borchers, and L. Thomas 2001. Introduction to DISTANCE SAMPLING: estimating abundance of biological populations. Oxford University Press, Oxford, New York etc.

Bunkus, E., M. Scheumann, and E. Zimmermann. 2005. Do captive-born grey mouse lemurs (Microcebus murinus) recognize their natural predators by acoustic cues? Primate Report 72.

Caswell, H. 1978. Predator-Mediated Coexistence: A Nonequilibrium Model. The American Naturalist 112:127154.

Cavender-Bares, J., D. D. Ackerly, D. A. Baum, and F. A. Bazzaz. 2004. Phylogenetic Overdispersion in Floridian Oak Communities. American Naturalist 163:823-843.

Cavender-Bares, J., and A. Wilczek. 2003. Integrating Micro- and Macroevolutionary Processes in Community Ecology. Ecology 84:592-597.

Charles-Dominique, P., and J. J. Petter. 1980. Ecology and Social Life of Phaner furcifer. Pages 75-96 in P. Charles-Dominique, H. M. Cooper, A. Hladik, C. M. Hladik, E. Pages, G. F. Pariente, A. PetterRousseaux, J. J. Petter, and A. Schilling, editors. Nocturnal Malagasy Primates. Academic Press, New York.

Chase, J. M., and M. A. Leibold 2003. Ecological niches. Linking classical and contemporary approaches. Interspecific Interactions. Univ. of Chicago Press.

Chesson, P. 2000a. Mechanisms and maintenance of species diversity. Annual Review of Ecological Systems 31:343-366.

Chesson, P. 2000b. General Theory of Competitive Coexistence in Spatially-Varying Environments. Theoretical Population Biology 58:211-237.

Chesson, P., and N. Huntly. 1997. The roles of harsh and fluctuating conditions in the dynamics of ecological communities. The American Naturalist 150:519-553.

Clements, F. E. 1936. Nature and structure of climax. Ecology 24:252-284.

Condit, R., N. Pitman, E. G. Leigh Jr., J. Chave, J. Terborgh, R. B. Foster, P. V. Núnez, S. Aguilar, R. Valencia, G. Villa, H. C. Muller-Landau, E. Losos, and S. P. Hubbell. 2002. Beta-Diversity in Tropical Forest Trees. Science 295:666-669.

Connell, J. H. 1980. Diversity and the coevolution of competitors, or the ghost of competition past. Oikos 35:131-138.

Connell, J. H., and R. O. Slatyer. 1977. Mechanisms of succession in natural communities and their role in community stability and organization. The American Naturalist 111:1119-1144.

Connor, E. F., and D. Simberloff. 1979. The Assembly of Species Communities: Chance or Competition? Ecology 60:1132-1140.

Connor, E. F., and D. Simberloff. 1983. Interspecific competition and species co-occurrence patterns on islands: Null models and the evaluation of evidence. Oikos 41:455-465.

Corbin, G. D., and J. Schmid. 1995. Insect secretions determine habitat use patterns by a female lesser mouse lemur (Microcebus murinus). American-Journal-of-Primatology 37:317-324.

Cornwell, W. K., D. W. Schwilk, and D. D. Ackerly. 2006. A trait-based test for habitat filtering: convex hull volume. Ecology 87:1465-1471.

Creel, S. 2001. Four factors modifying the effect of competition in carnivore population dynamics as illustrated by African wild dogs. Conservation Biology 15:271-274.

Creel, S., and N. M. Creel. 1996. Limitation of African wild dogs by competition with larger carnivores. Conservation Biology 10:526-538.

Dammhahn, M., and P. M. Kappeler. 2005. Social system of Microcebus berthae, the world's smallest primate. International Journal of Primatology 26:407-435.

Dammhahn, M., and P. M. Kappeler. 2008. Small-scale coexistence of two mouse lemur species (Microcebus berthae and $M$. murinus) within a homogeneous competitive environment. Oecologia (Berlin) 157:473483.

Dammhahn, M., and P. M. Kappeler. 2009. Females go where the food is: does the socio-ecological model explain variation in social organization of solitary foragers? Behavioral Ecology and Sociobiology 63:393-352.

Dammhahn, M., and P. M. Kappeler. 2010. Scramble or Contest Competition Over Food in Solitarily Foraging Mouse Lemurs (Microcebus spp.): New Insights From Stable Isotopes. American Journal of Physical Anthropology 141:181-189. 
Danielson, B. J. 1992. Habitat selection, interspecific interactions and landscape composition. Evolutionary Ecology 6:399-411.

Dausmann, K. H., J. Glos, J. U. Ganzhorn, and G. Heldmaier. 2004. Hibernation in a tropical primate. Nature 429:825-826.

Deppe, A. 2005. Visual predator recognition and response in wild brown mouse lemurs (Microcebus rufus) in Ranomafana National Park, Madagascar. ASP Conference. The American Society of Primatologists.

Deppe, A., M. Randiramiarisoa, and P. C. Wright. 2007. Mammalian predator recognition via olfactory cues in wild brown mouse lemurs (Microcebus rufus). American Journal of Physical Anthropology Suppl. 44:99.

Deppe, A., and P. C. Wright. 2009. Predator recognition in wild brown mouse lemurs (Microcebus rufus): field experiments in Ranomafana National Park, Madagascar. ASP Symposium. American Society of Primatologists.

Diamond, J. M. 1975. Assembly of species communities. Pages 342-444 in M. L. Cody, and J. M. Diamond, editors. Ecology and evolution of communities. Harvard University Press, Cambridge.

Diamond, J. M. 1980a. Species turnover in island bird communities. Acta Congressus Internationalis Ornithologici 2:777-782.

Diamond, J. M. 1980b. Why are many tropical bird species distributed patchily with respect to available habitat? Acta Congressus Internationalis Ornithologici 2:968-973.

Dinerstein, E. et al. 1996. The Global 200: Key Ecoregions for Saving Life on Earth. World Wide Fund for Nature, Washington, DC.

Eberle, M., and P. M. Kappeler. 2002. Mouse lemurs in space and time: a test of the socioecological model. Behavioral-Ecology-and-Sociobiology 51:131-139.

Eberle, M., and P. M. Kappeler. 2004a. Sex in the dark: determinants and consequences of mixed male mating tactics in Microcebus murinus, a small solitary nocturnal primate. Behavioral-Ecology-and-Sociobiology 57:77-90.

Eberle, M., and P. M. Kappeler. 2004b. Selected polyandry: female choice and inter-sexual conflict in a small nocturnal solitary primate (Microcebus murinus). Behavioral Ecology and Sociobiology 57:91-100.

Eberle, M. E., and P. M. Kappeler. 2006. Family insurance: kin selection and cooperative breeding in a solitary primate (Microcebus murinus). Behavioral Ecology and Sociobiology 60:582-588.

Engelbrecht, B. M. J., L. S. Comita, R. Condit, T. A. Kursar, M. T. Tyree, B. L. Turner, and S. P. Hubbell. 2007. Drought sensitivity shapes species distribution patterns in tropical forests. Nature 447:80-82.

Enquist, B. J., M. A. Jordan, and J. H. Brown. 1995. Connection between ecology, biogeography, and paleobiology: relationship between local abundance and geographic distribution in fossil and recent molluscs. Evolutionary Ecology 9:586-604.

Estes, J. A., and J. F. Palmisano. 1974. Sea Otter: Their Role in Structuring Nearshore Communities. Science 185:1058-1060.

Estes, J. A., J. Terborgh, J. S. Brashares, M. E. Power, J. Berger, W. J. Bond, S. R. Carpenter, T. E. Essington, R. D. Holt, J. B. C. Jackson, R. J. Marquis, L. Oksanen, T. Oksanen, R. T. Paine, E. K. Pikitch, W. J. Ripple, S. A. Sandin, M. Scheffer, T. W. Schoener, J. B. Shurin, A. R. E. Sinclair, M. E. Soulé, R. Virtanen, and D. A. Wardle. 2011. Trophic Downgrading of Planet Earth. Science 333:301-306.

Fariña, J. M., B. R. Silliman, and M. D. Bertness. 2009. Can conservation biologists rely on established community stracture rules to manage novel systems? ... Not in salt marshes. Ecological Applications 19:413-422.

Fietz, J. 1999. Monogamy as a Rule Rather than Exception in Nocturnal Lemurs: the Case of the Fat-tailed Dwarf Lemur, Cheirogaleus medius. Ethology 105:259-272.

Fietz, J. 2003. Primates: Cheirogaleus, Dwarf Lemurs or Fat-tailed Lemurs in S. M. Goodman, and J. P. Benstead, editors. Natural History of Madagascar. University Chicago Press, Chicago.

Fichtel, C. 2009. Costs of alarm calling: lemur alarm calls attract fossas. Lemur News 14:53-55.

Fischer, J., and D. B. Lindenmayer. 2007. Landscape modification and habitat fragmentation: a synthesis. Global Ecology and Biogeography 16:265-280.

Fischer, L. A., and C. C. Gates. 2005. Competition potential between sympatric woodland caribou and wood bison in southwestern Yukon, Canada. Canadian Journal of Zoology 83:1162-1173.

Fredsted, T., C. Pertoldi, J. M. Olesen, M. Eberle, and P. M. Kappeler. 2004. Microgeographic heterogeneity in spatial distribution and mtDNA variability of grey mouse lemurs (Microcebus murinus, Primates: Cheirogaleidae). Behav Ecol Sociobiol 56:393-403.

Fredsted, T., C. Pertoldi, M. H. Schierup, and P. M. Kappeler. 2005. Microsatellite analyses reveal fine-scale genetic structure in grey mouse lemurs (Microcebus murinus). Molecular Ecology 14:2363-2372. 
Fredsted, T., M. H. Schierup, G. L. Fenna, and K. P. M. 2007. Genetic structure, lack of sex-biased dispersal and behavioral flexibility in the pair-living fat-tailed dwarf lemur, Cheirogaleus medius. Behavioral Ecology and Sociobiology 61:943-954.

Fukami, T., T. M. Bezemer, S. R. Mortimer, and W. H. van der Putten. 2005. Species divergence and trait convergence in experimental plant community assembly. Ecology Letters 8:1283-1290.

Ganzhorn, A. W. 2002. Distribution of a folivorous lemur in relation to seasonally varying food resources: integrating quantitative and qualitative aspects of food characteristics. Oecologia (Berlin) 131:427435.

Ganzhorn, A. W. 2003. Habitat description and phenology. Pages 40-56 in J. M. Setchell and D. J. Curtis, editors. Field and Laboratory Methods in Primatology. Cambridge University Press, Cambridge.

Ganzhorn, A. W., and J. Schmid. 1998. Different Population Dynamics of Microcebus murinus in Primary and Secondary Deciduous Dry Forests of Madagascar. International Journal of Primatology 19:785-796.

Ganzhorn, J. 1994. Lemurs as indicators for habitat change. Pages 51-56 in B. Thierry, J. R. Anderson, U. Roeder \& N. Herrenschmidt, editors. Current Primatology: Ecology and Evolution. Universite Louis Pasteur, Strassbourg, France.

Ganzhorn, J. 1995. Low-Level Forest Disturbance Effect on Primary Production, Leaf Chemistry, and Lemur Populations. Ecology 76:2084-2096.

Ganzhorn, J. U. 1987. A Possible Role of Plantations for Primate Conservation in Madagascar. Am. J. Primatol. 12:205-215.

Ganzhorn, J. U. 1991. Tier- und Artenschutz aus der Sicht des Biotopschutzes: Eine Fallstudie aus dem Trockenwald Madagaskars. Pages 109-120 in H. Rahmann, and A. Kohler, editors. Tier- und Artenschutz. Margraf Verlag, Weikersheim.

Ganzhorn, J. U. 1999. Lemurs as indicators for assessing biodiversity in forest ecosystems of madagascar: Why it does not work. Tasks for vegetation science 34:163-174.

Ganzhorn, J. U., and B. Eisenbeiß. 2001. The the concept of nested species assemblages and its utility for understanding effects of habitat fragmentation. Basic Appl. Ecol. 2, 87-95 (2001) 2:87-95.

Ganzhorn, J. U., J. Fietz, E. Rakotovao, D. Schwab, and D. Zinner. 1999. Lemurs and the regeneration of dry deciduous forest in Madagascar. Conservation Biology 13:794-804.

Ganzhorn, J. U., A. W. Ganzhorn, J. P. Abraham, L. Andriamanarivo, and A. Ramananjatovo. 1990. The impact of selective logging on forest structure and tenrec populations in western Madagascar. Oecologia (Berlin) 84:126-133.

Ganzhorn, J. U., S. M. Goodman, and A. Dehgan. 2003. Effects of forest fragmentation on small mammals and lemurs. Pages 1228-1234 in S. M. Goodman, and J. P. Benstead, editors. The Natural History of Madagascar. University Chicago Press, Chicago.

Ganzhorn, J. U., and P. M. Kappeler. 1996. Lemurs of the Kirindy forest. Primate Report 46:257-274.

Ganzhorn, J. U., P. P. Lowry II, G. E. Schatz, and S. Sommer. 2001. The biodiversity of Madagascar: one of the world's hottest hotspots on its way out. Oryx 35:346-348.

Ganzhorn, J. U., S. Malcomber, O. Andrianantoanina, and S. M. Goodman. 1997. Habitat characteristics and lemur species richness in Madagascar. Biotropica 29:331-343.

Gardner, T. A., J. Barlow, R. Chazdon, R. M. Ewers, C. A. Hervey, C. A. Peres, and N. S. Sodhi. 2009. Prospects for tropical forest biodiversity in a human-modified world. Ecology Letters 12:561-582.

Gascon, C., T. E. Lovejoy, R. O. Bierregaard Jr., J. R. Malcom, P. C. Stouffer, H. L. Vasconcelos, W. F. Laurance, B. Zimmermann, M. Tocher, and S. Borges. 1999. Matrix habitat and species richness in tropical forest remnants. Biological Conservation 91:223-229.

Gibbons, M. A., and A. H. Harcourt. 2009. Biological correlates of extinction and persistence of primates in small forest fragments: a global analysis. Tropical Conservation Science 2:388-403.

Glasser, J. W. 1979. The role of predation in shaping and maintaining the structure of communities. American Naturalist 113:631-641.

Glasser, J. W. 1983. Variation in Niche Breadth with Trophic Position: On the Disparity between Expected and Observed Species Packing. The American Naturalist 122:542-548.

Goodman, S. M. 2003. Predation on Lemurs. Pages 1221-1228 in S. M. Goodman, and J. P. Benstead, editors. The Natural History of Madagascar. University of Chicago Press, Chicago.

Goodman, S. M., S. O'Connor, and O. Langrand. 1993. A review of predation on lemurs: implications for the evolution of social behavior in small, nocturnal primates. Pages 51-66 in -. P.-M. Kappeler, and J. U. Ganzhorn, editors. Lemur social systems and their ecological basis. Plenum Press, New York \& London.

Gotelli, N. J. 2000. Null Model Analysis of Species Co-Occurrence Patterns. Ecology 81:2606-2621. 
Gotelli, N. J., and G. L. Entsminger. 2011. EcoSim: Null models software for ecology. Version 7 in http://garyentsminger.com/ecosim.htm, editor. Acquired Intelligence Inc. \& Kesey-Bear., Jericho, VT 05465.

Gotelli, N. J., and G. L. Entsminger. 2004. EcoSim: Null models software for ecology. Version 7. Acquired Intelligence Inc. \& Kesey-Bear, Jericho, VT 05465.

Gotelli, N. J., and D. J. McCabe. 2002. Species co-occurrence: A meta-analysis of J. M. Diamond's assembly rules model. Ecology 83:2091-2096.

Green, G. M., and R. W. Sussman. 1990. Deforestation history of the eastern rain forest of Madagascar from satellite images. Science 248:212-215.

Gregorius, H.-R. 1996. The Contribution of the Genetics of Populations to Ecosystem Stability. Silvae Genetica 45:267-271.

Hannah, L., D. Radhika, P. P. Lowry II, S. Andelman, M. Andrianarisata, L. Andriamaro, A. Cameron, R. Hijmans, C. Kremen, J. MacKinnon, H. H. Randrianasolo, S. Andriambololonera, A. Razafimpahanana, H. Randriamahazo, J. Randrianarisoa, P. Razafinjatovo, C. Raxworthy, G. E. Schatz, M. Tadross, and L. Wilmé. 2008. Climate change adaptation for conservation in Madagascar. Biology Letters 4:590-594.

Hanski, I. 1997. Metapopulation dynamics: from concepts and observations to to predictive models. Pages 6992 in I. Hanski, and M. E. Gilpin, editors. Metapopulation Biology. Academic Press, San Diego, CA.

Hanski, I., and E. Ranta. 1983. Coexistence in a patchy environment: three species of Daphnia in rock pools. Journal of Animal Ecology 52:263-279.

Hanski, I., and D. Simberloff. 1997. The metapopulation approach, its history, conceptual domain and application to conservation. Pages 5-26 in I. Hanski, and M. E. Gilpin, editors. Metapopulation biology. Academic Press, San Diego.

Hardin, G. 1960. The competitive exclusion principle. Science 131:1292-1297.

Hart, D. 2007. Predation on Primates: A Biogeographical Analysis. Pages 27-59 in S. L. Gursky, and K. A. I. Nekaris, editors. Primate Anti-predator Strategies. Springer Science+Business Media, New York.

Harvey, C. A., A. Medina, D. Sánchez Merlo, S. Vílchez, B. Hernández, J. Saenz, J. Maes, F. Casanovas, and F. L. Sinclair. 2006. Patterns of animal diversity associated with different forms of tree cover retained in agricultural landscapes. Ecological Applications $\mathbf{1 6 .}$

Hastings, A. 1987. Can competition be detected using species co-occurrence data? Ecology 68:117-123.

Hattori, A. 2002. Small and large anemone fishes can coexist using the same patchy resources on a coral reef, before habitat destruction. Journal-of-Animal-Ecology 71:824-831.

Hauser, M. D., and R. W. Wrangham. 1990. Recognition of predator and competitor calls in nonhuman primates and birds: a preliminary report. Ethology 86:116-130.

Herrera, A., J. B. C. Jackson, D. J. Hughes, J. Jara, and H. Ramos. 1996. Life-history variation in three coexisting cheilostome bryozoan species of the genus Sfylopoma in Panama. Marine Biology 126:461- 469.

Hickerson, C.-A. M., C. D. Anthony, and J. A. Wicknick. 2004. Behavioral interactions between salamanders and centipedes: competition in divergent taxa. Behavioral Ecology 15:679-686.

Hladik, C. M., P. Charles-Dominique, and J. J. Petter. 1980. Feeding Strategies of five nocturnal prosimians in the dry deciduous forest of the west coast of Madagascar. Pages 41-74 in P. Charles-Dominique, H. M. Cooper, A. Hladik, C. M. Hladik, E. Pages, G. F. Pariente, A. Petter-Rousseaux, J. J. Petter, and A. Schilling, editors. Nocturnal Malagasy Primates. Academic Press, New York.

Holt, R. D. 1984. Spatial heterogeneity, indirect interactions, and the coexistence of prey species. American Naturalist 124:377-406

Holt, R. D., J. Grover, and D. Tilman. 1994. Simple rules for interspecific dominance in systems with exploitative and apparent competition. The American Naturalist 144:741-771.

Holt, R. D., and J. H. Lawton. 1994. The Ecological Consequences of Shared Natural Enemies. Annual Review of Ecology and Systematics 25:495-520.

Holt, R. D., and G. A. Polis. 1997. A theoretical framework for intraguild predation. The American Naturalist 149:745-764.

Houle, A. 1997. The role of phylogeny and behavioral competition in the evolution of coexistence among primates. Canadian Journal of Zoology 75:827-846.

Howe, R. W., and G. J. Davis. 1991. The Demographic Significance of 'Sink' Populations. Biological Conservation 57:239-255.

Hubbell, S. P. 1997. A unified theory of biogeography and relative species abundance and its application to tropical rain forests and coral reefs. Coral Reefs 16:9-21.

Hutchinson, G. E. 1959. Homage to Santa Rosalia, or why are there so many kinds of animals? American Naturalist 93:145-159. 
Ims, R. A., and N. G. Yoccoz. 1997. Studying transfer processes in metapopulations: emigration, migration, and colonization. Pages 247-265 in I. Hanski, and M. E. Gilpin, editors. Metapopulation biology. Ecology, genetics and evolution. Academic Press, San Diego, CA.

Irwin, M. T., and J.-L. Raharison. 2009. Ecosystems in decay: factors influencing primate species extinctions in forest fragments of Tsinjoarivo, Madagascar. American Journal of Physical Anthropology 138:155.

Irwin, M. T., J.-L. Raharison, and P. C. Wright. 2009. Spatial and temporal variability in predation on rainforest primates: do forest fragmentation and predation act synergistically? Animal Conservation 12:220-230.

Irwin, M. T., P. C. Wright, C. Birkinshaw, B. L. Fisher, C. J. Gardner, J. Glos, S. M. Goodman, P. Loiselle, P. Rabeson, J.-L. Raharison, M. J. Raherilalao, D. Rakotondravony, A. Raselimanana, J. Razimbazafy, J. S. Sparks, L. Wilmé, and J. U. Ganzhorn. 2010. Patterns of species change in anthropogenically disturbed forests of Madagascar. Biological Conservation *early view*.

Jensen, A. L. 1997. Limiting similarity for coexistence of lake herring (Coregonus artedii) and chubs (Coregonus hoyi). Ecological Modelling 95:11-15.

Kappeler, P. M. 2003. Mirza coquereli, Coquerel's dwarf lemur. Pages 1316-1318 in S. M. Goodman, and J. P. Benstead, editors. The Natural History of Madagascar. University Chicago Press, Chicago.

Kappeler, P. M., B. Wimmer, D. Zinner, and D. Tautz. 2002. The hidden matrilineal structure of a solitary lemur: implications for primate social evolution. Proceedings of the Royal Society: Biological Sciences - Series B 269:1755 -- 1763.

Kareiva, P. 1990. Population dynamics in a spatially complex environment: theory and data. Philosophical Transactions of the Royal Society of London, Series B 330:175-190.

Karpanty, S. M., and P. C. Wright. 2007. Predation on Lemurs in the Rainforest of Madagascar by Multiple Predator Species: Observations and Experiments. Pages 77-99 in S. L. Gursky, and K. A. I. Nekaris, editors. Primate Anti-predator Strategies. Springer Science+Business Media, New York.

Klopfer, P. H. 1977. Communication in Prosimians. Pages 841-850 in T. A. Sebeok, editor. How animals communicate. Indiana University Press, Bloomington \& London.

Kozakiewics, M. 1993. Habitat selection and ecological barriers - the effects on small mammal populations and communities. Acta Theriologica 38:1-30.

Kraft, N. J. B., R. Valencia, and D. D. Ackerly. 2008. Functional Traits and Niche-Based Tree Community Assembly in an Amazonian Forest. Science 322:580-582.

Kullberg, C., and J. Eckman. 2000. Does predation maintain tit community diversity? Oikos 89:41-45.

Lahann, P. 2008. Habitat Utilization of Three Sympatric Lemur Species in a Littorial Rain Forest of Southeastern Madagascar. International Journal of Primatology 29:117-134.

Lahann, P., J. Schmid, and J. U. Ganzhorn. 2006. Geographic Variation in Populations of Microcebus murinus in Madagascar: Resource Seasonality or Bergmann's Rule? Int. J. of Primatol. 27:983-999.

Lande, R. 1988. Genetics and Demography in Biological Conservation. Science 241:1455-1460.

Laurance, W. F. 1991. Ecological correlates of extinction proneness in Australian tropical rain forest mammals. Conservation Biology 5:79-89.

Lawes, M. J., P. E. Mealin, and S. E. Piper. 2000. Patch Occupancy and Potential Metapopulation Dynamics of Three Forest Mammals in Fragmented Afromontane Forest in South Africa. Conservation Biology 14:1088-1098.

Leaver, L. A., and M. Daly. 2001. Food caching and differential cache pilferage: a field study of coexistence of sympatric kangaroo rats and pocket mice. Oecologia (Berlin) 128:577-584.

Lehman, S. M. 2006a. Conservation Biology of Malagasy Strepsirhines: A Phylogenetic Approach. American Journal of Physical Anthropology 130:238-253.

Lehman, S. M. 2006b. Effects of Transect Selection and Seasonality on Lemur Density Estimates in Southeastern Madagascar. International Journal of Primatology 27:1041-1057.

Lehman, S. M., A. Rajaonson, and S. Day. 2006a. Edge Effects and Their Influence on Lemur Density and Distribution in Southeast Madagascar. American Journal of Physical Anthropology 129:232-241.

Lehman, S. M., A. Rajaonson, and S. Day. 2006b. Edge Effects on the Density of Cheirogaleus major. International Journal of Primatology 27:1569-1588.

Leibold, M. A., M. Holyoak, N. Mouquet, P. Amarasekare, J. M. Chase, M. F. Hoopes, R. D. Holt, J. B. Hurin, R. Law, D. Tilman, M. Loreau, and A. Gonzalez. 2004. The metacommunity concept: a framework for multi-scale community ecology. Ecology-Letters 7:601-613.

Lessard, J. P., J. A. Fordyce, N. J. Gotelli, and N. J. Sanders. 2009. Invasive ants alter the phylogenetic structure of ant communities. Ecology 90:2664-2669.

Letcher, A. J., A. Purvis, S. Nee, and P. H. Harvey. 1994. Patterns of Overlap in the Geographic Ranges of Palearctic and British Mammals. Journal of Animal Ecology 63:871-879. 
Lindenmayer, D. B., C. R. Margules, and D. B. Botkin. 2000. Indicators of Biodiversity for Ecologically Sustainable Forest Management. Conservation Biology 14:941-950.

Linnell, J. D. C., and O. Strand. 2000. Interference interactions, co-existence and conservation of mammalian carnivores. Diversity and Distributions 6:169-176.

López-Gómez, J., and M. Molina-Meyer. 2006. The competitive exclusion principle versus biodiversity through competitive segregation and further adaptation to spatial heterogeneities. Theoretical Population Biology 69:94-109.

Lotka, A. J. 1925. Elements of Physical Biology. Williams and Wilkins, Baltimore.

Lowry II, P. P., G. E. Schatz, and P. B. Phillipson. 1997. The classification of natural and anthropogenic vegetation in Madagascar. Pages 93-123 in S. M. Goodman, and B. D. Patterson, editors. Natural Change and Human Impact in Madagascar. Smithsonian Institution Press, Washington and London.

MacArthur, R. H., and R. Levins. 1967. The limiting similarity, convergence and divergence of coexisting species. American Naturalist 101:377-385.

Markolf, M., C. Roos, and P. M. Kappeler. 2008. Genetic and Demographic Consequences of a Rapid Reduction in Population Size in a Solitary Lemur (Mirza coquereli). The Open Conservation Biology Journal 2:2129.

Martin, R. D. 1972. A preliminary field study of the Lesser Mouse Lemur (Microcebus murinus, J. F. Miller 1777). Zeitschr. Tierpsych. Beiheft:43-89.

May, R. M. 1981. Models for two interacting populations. Pages 78-104 in R. M. May, editor. Theoretical Ecology: Principles \& Applications. Blackwell Scientific Publications, Oxford.

Mevanarivo, Z. E. 2009. Caractéristiques de la forêt dense sèche de Kirindy et de ses environs comme habitat naturel de Microcebus berthae. Département des Eaux et Forêts, Ecoles Supérieur des Sciences Agronomiques. Université d'Antananarivo, Antananarivo, Madagascar.

Miller, A. D., S. H. Roxburgh, and K. Shea. 2011. How frequency and intensity shape diversity-disturbance relationships. PNAS 108:5643-5648.

Mittermeier, R. A., W. R. Konstant, M. E. Nicoll, and O. Langrand 1992. Lemurs of Madagascar: An Action Plan for their Conservation, 1993-1999, Gland, Switzerland.

Mittermeier, R. A., J. U. Ganzhorn, W. R. Konstant, K. Glander, I. Tattersall, C. P. Groves, A. B. Rylands, A. Hapke, J. Ratsimbazafy, M. I. Mayor, E. E. L. Jr., Y. Rumpler, C. Schwitzer, and R. M. Rasoloarison. 2008. Lemur Diversity in Madagascar. International Journal of Primatology 29:1607-1656.

Morin, P. J., S. P. Lawler, and E. A. Johnson. 1988. Competition between Aquatic Insects and Vertebrates: Interaction Strength and Higher Order Interactions. Ecology 69:1401-1409.

Murrell, D. J., and R. Law. 2003. Heteromyopia and the spatial coexistence of similar competitors. Ecology Letters 6:48-59.

Myers, N., R. A. Mittermeier, C. G. Mittermeier, G. A. B. da Fonseca, and J. Kent. 2000. Biodiversity hotspots for conservation priorities. Nature 403:853-858.

Namgail, T., J. L. Fox, and Y. V. Bhatnagar. 2004. Habitat segregation between sympatric Tibetan argali Ovis ammon hodgsoni and blue sheep Pseudois nayaur in the Indian Trans-Himalaya. Journal of Zoology 262:57-63.

Nie, N. H., C. H. Hull, J. G. Jenkins, K. Steinbrenner, and D. H. Bent. 1975. SPSS. Statistical Package for the Social Sciences/ SPSS-Statistics. 2008. SPSS for Windows Release 17.0. SPSS Inc., Chicago.

McGraw-Hill, , New York, NY.

Nupp, T. E., and R. K. Swihart. 2001. Assessing competition between forest rodents in a fragmented landscape of midwestern USA. Mammalian Biology 66:345-356.

Olivieria, G. L., V. Sousab, L. Chikhib, and U. Radespiel. 2008. From genetic diversity and structure to conservation: Genetic signature of recent population declines in three mouse lemur species (Microcebus spp.). Biological Conservation 141:1257-1271.

Pages, E. 1980. Ethoecology of Microcebus coquereli during the dry season. Pages 97-116 in P. CharlesDominique, H. M. Cooper, A. Hladik, C. M. Hladik, E. Pages, G. F. Pariente, A. Petter-Rousseaux, J. J. Petter, and A. Schilling, editors. Nocturnal Malagasy Primates. Academic Press, New York.

Paine, R. T. 1966. Food Web Complexity and Species Diversity. American Naturalist 100:65-75.

Paine, R. T. 1974. Intertidal Community Structure. Oecologia (Berlin) 15:93-120.

Pardini, R., S. Marques de Souza, R. Braga-Neta, and J. P. Metzger. 2005. The role of forest structure, fragment size and corridors in maintaining small mammal abundance and diversity in an Atlantic forest landscape. Biological Conservation 124:253-266.

Pertraitis, P. S., E. T. Methratta, E. C. Rhile, N. A. Vidargas, and S. R. Dudgeon. 2009. Experimental confirmation of multiple community states in a marine ecosystem. Oecologia (Berlin) 161:139-148. 
Petchey, O. L., A. Gonzalez, and H. B. Wilson. 1997. Effects on population persistence: the interaction between environmental noise color, intraspecific competition and space. Proceedings of the Royal Society London, Series B 264:1841-1847.

Petter, J. J., R. Albignac, and Y. Rumpler. 1977. Mammifères lémuriens (Primates, Prosimiens) in ORSTROMCNRS, editor. Faune de Madagascar No. 44, Paris.

Petter-Rousseaux, A. 1980. Seasonal activity rythms, reproduction, and body weight variations in five sympatric nocturnal prosimians, simulated light and climatic conditions. Pages 137-152 in P. Charles-Dominique, H. M. Cooper, A. Hladik, C. M. Hladik, E. Pages, G. F. Pariente, A. Petter-Rousseaux, J. J. Petter, and A. Schilling, editors. Nocturnal Malagasy Primates:Ecology, Physiology and Behavior. Academic Press, New York.

Philpott, S. M., P. Bichler, R. A. Rice, and R. Greenberg. 2008. Biodiversity Conservation, yield, and alternative products in coffee agroecosystems in Sumatra, Indonesia. Biodiversity and Conservation 17:18051820.

Pianka, E. R. 1973. The structure of lizzard communities. Annual Review of Ecological Systems 4:53-74.

Pinkus-Rendón, M. A., J. L. Léon-Cortés, and G. Ibarra-Núnez. 2006. Spider diversity in a tropical habitat gradient in Chiapas, Mexico. Diversity and Distributions 12:61-69.

Polis, G. A., and R. D. Holt. 1992. Intraguild Predation: The Dynamics of Complex Trophic Interactions. Trends in Ecology and Evolution 7:151-155.

Polis, G. A., C. A. Myers, and R. D. Holt. 1989. The ecology and evolution of intraguild predation. Annual Review of Ecology and Systematics 20:297-330.

Pozo, R., and E. Wilmer. 2004. Habitat preferences of six non-Atelidae primates of Yasuni, Ecuador. Ecologia Aplicada 3:128-133.

Rahlfs, M., and C. Fichtel. 2010. Anti-Predator Behaviour in a Nocturnal Primate, the Grey Mouse Lemur (Microcebus murinus). Ethology 116:429-439.

Rakotonirainy, E. O. 2003. Etude de la prédation entre Mirza coquereli (A. Grandidier, 1867) et Microcebus murinus (J.F. Miller, 1777) dans la forêt de Kirindy, Morondava, Madagascar. Lemur News 8:29.

Randrianandianina, B. N., L. R. Andriamahaly, F. M. Harisoa, and M. E. Nicoll. 2003. The role of protected areas in the management of the island's biodiversity. Pages 1423-1432 in S. M. Goodman, and J. P. Benstead, editors. The Natural History of Madagascar. University Chicago Press, Chicago.

Rasoloarison, R. M., S. M. Goodman, and A. W. Ganzhorn. 2000. Taxonomic Revision of Mouse Lemurs (Microcebus) in the Western Portions of Madagascar. Int. J. of Primatol. 21:963-1019.

Rasoloarison, R. M., B. P. N. Rasolonandrasana, J. U. Ganzhorn, and S. M. Goodman. 1995. Predation on vertebrates in the Kirindy Forest, western Madagascar. Ecotropica 1:59-65.

R-Development-Core-Team. 2005. R: A language and environment for statistical computing in R-Foundationfor-Statistical-Computing, editor. 2.11.1, Vienna, Austria.

Réau, B. 2002. Burning for zebu: the complexity of deforestation issues in western Madagascar. Norwegian Journal of Geography 56:219-229.

Reichholf, J. H. 1993. Comeback der Biber. Ökologische Überraschungen. C. H. Beck'sche Buchhandlung, München.

Reimann, W. E. 2002. Koexistenz und Nahrungsökologie von eibchen des grauen und goldbraunen Mausmaki (Microcebus murinus und M. ravelobensis) in Nordwest-Madagaskar. Page 216. Institut für Zoologie. Tierärztliche Hochschule, Hannover.

Reimann, W., U. Radespiel, and E. Zimmermann. 2003. Feeding regimes of two sympatric mouse lemurs in north-western Madagascar (Microcebus murinus and M. ravelobensis): No clear evidence for niche separation. Folia Primatol. 74:215-216.

Rendigs, A., U. Radespiel, D. Wrogemann, and E. Zimmermann. 2003. Relationship Between Microhabitat Structure and Distribution of Mouse Lemurs (Microcebus spp.) in Northwestern Madagascar. Int. J. of Primatol. 24:47-64.

Roughgarden, J. 1974. Population dynamics in a spatially varying environment: How population size "tracks" spatial variation in carrying capacity. The American Naturalist 108:649-664.

Sachot, S., N. Perrin, and C. Neet. 2003. Winter habitat selection by two sympatric forest grouse in western Switzerland: implications for conservation. Biological Conservation 112.

Sanderson, J. G. 2004. Null model analysis of communities on gradients. Journal of Biogeography 31:879-883.

Sanderson, J. G., J. M. Diamond, and S. L. Pimm. 2009. Pairwise co-existence of Bismarck and Solomon landbird species. Evolutionary Ecology Research 11:771-786.

Sandersson, J. G. 2004. Null model analysis of communities on gradients. Journal of Biogeography 31:879-883.

Sarre, S., G. T. Smith, and J. A. Meyers. 1995. Persistence of two species of gecko (Oedura reticulata and Gehyra variegata) in remnant habitat. Biological Conservation 71:25-33. 
Scheumann, M., A. Rabesandratana, and E. Zimmermann. 2007. Predation, Communication, and Cognition in Lemurs. Pages 100-126 in S. L. Gursky, and K. A. I. Nekaris, editors. Primate Anti-predator Strategies. Springer Science+Business Media, New York.

Schliehe-Diecks, S., M. Markolf, and E. Huchard. 2010. When big lemurs swallow up small ones: Coquerel's dwarf lemur as a predator of grey mouse lemurs and endemic rodents. Lemur News 15:13-14.

Schmid, J. 1996. Oxygen consumption and torpor in mouse lemurs (Microcebus murinus and M. myoxinus): preliminary results of a study in western Madagascar. Pages 47-54 in F. Geiser, A. Hulbert, and S. Nicol, editors. Adaptations to the cold: Tenth International Hibernation Symposium. University of New England Press, Armidale.

Schmid, J. 1999. Sex-specific differences in activity patterns and fattening in the gray mouse lemur (Microcebus murinus) in Madagascar. Journal of Mammalogy 80:749-757.

Schmid, J. 2000a. Torpor in the tropics: the case of the gray mouse lemur (Microcebus murinus). Basic-andApplied-Ecology 1:133-139.

Schmid, J. 2000b. Daily torpor in the gray mouse lemur (Microcebus murinus) in Madagascar: energetic consequences and biological significance. Oecologia (Berlin) 123:175-183.

Schmid, J. 2001. Daily torpor in free-ranging gray mouse lemurs (Microcebus murinus) in Madagascar. International Journal of Primatology 22:1021-1031.

Schmid, J., and P. M. Kappeler. 1994. Sympatric mouse lemurs (Microcebus spp.) in Western Madagascar. Folia Primatol. 63.

Schmid, J., and P. M. Kappeler. 1998. Fluctuating sexual dimorphism and differential hibernation by sex in a primate, the gray mouse lemur (Microcebus murinus). Behavioral Ecology and Sociobiology 43:125132.

Schmid, J., T. Ruf, and G. Heldmaier. 2000. Metabolism and temperature regulation during daily torpor in the smallest primate, the pygmy mouse lemur (Microcebus myoxinus) in Madagascar. Journal of Comparative Physiology B Biochemical Systemic and Environmental Physiology 170:59-68.

Schmid, J., and J. R. Speakman. 2000. Daily energy expenditure of the grey mouse lemur (Microcebus murinus): a small primate that uses torpor. Journal-of-Comparative-Physiology-B-Biochemical-Systemic-andEnvironmental-Physiology 170:633-641.

Schoener, T. W. 1983. Field experiments on interspecific competition. American Naturalist 122:240-285.

Schoener, T. W., and D. A. Spiller. 1987. High population persistence in a system with high turnover. Nature 330:474-477.

Schreiber, S. J., and M. Kelton. 2005. Sink habitats can alter ecological outcomes for competing species. Journal of Animal Ecology 74:995-1004.

Schülke, O. 2001. Social Anti-Predator Behaviour in a Nocturnal Lemur. Folia Primatol. 72:332-334.

Schulze, C. H., M. Waltert, P. J. A. Kessler, R. Pitopang, Shahabuddin, D. Veddeler, M. Mühlenberg, S. R. Gradstein, C. Leuschner, I. Steffan-Dewenter, and T. Tscharntke. 2004. Biodiversity indicator groups of tropical land-use systems: comparing plants, birds, and insects. Ecological Applications 14:1321-1333.

Schwab, D., and J. U. Ganzhorn. 2004. Distribution, population structure and habitat use of Microcebus berthae compared to those of other sympatric Cheirogaleids. International Journal of Primatology 25:307-330.

Schweiger, E. W., J. E. Diffendorfer, R. Pierotti, and R. D. Holt. 1999. The Relative Importance of Small-Scale and Lanscape-Level Heterogeneity in Structuring Small Mammal Distributions. Pages 175-207 in G. W. Barret, and J. D. Peles, editors. Landscape Ecology of Small Mammals. Springer, New York.

Seidl, I., and C. A. Tisdell. 1999. Carrying capacity reconsidered: from Malthus' population theory to cultural carrying capacity. Ecological Economics 31:395-408.

Sfenthourakis, S., E. Tzanatos, and S. Giokas. 2005. Species co-occurrence: the case of congeneric species and a causal approach to patterns of species association. Global Ecology and Biogeography 15:39-49.

Slingsby, J. A., and G. A. Verboom. 2006. Phylogenetic relatedness limits co-occurrence at fine spatial scales: Evidence from the schoenoid sedges (Cyperaceae: Schoeneae) of the cape floristic region, South Africa. American Naturalist 168:14-27.

Smith, A. P., and J. U. Ganzhorn. 1996. Convergence in community structure and dietary adaptation in Australian possums and gliders and Malagasy lemurs. Australian-Journal-of-Ecology 21:31-46.

Smith, A. P., N. Horning, and D. Moore. 1997. Regional Biodiversity Planning and Lemur Conservation with GIS in Western Madagascar. Conservation Biology 11:498-512.

Snyder, R. E., and P. Chesson. 2003. Local dispersal can facilitate coexistence in the presence of permanent spatial heterogeneity. Ecology Letters 6:301-309.

Sokal, R. R., and F. J. Rohlf. 1981. Taxonomic congruence in the Leptopodomorpha re-examined. Systematic Zoology 30:309-325. 
Sorg, J. P., J. U. Ganzhorn, and P. M. Kappeler. 2003. Forestry and research in the Kirindy Forest/ Centre de Formation Professionnelle Forestière. Pages 1512-1519 in S. M. Goodman, and J. P. Benstead, editors. The Natural History of Madagascar. University Chicago Press, Chicago.

Sorg, J. P., and U. Rohner. 1996. Climate and tree phenology of the dry deciduous forest of the Kirindy Forest. Primate Report 46-1: Ecology and Economy of a Tropical Dry Forest in Madagascar.

Soulé, M. E., B. G. Mackey, H. F. Recher, J. E. Williams, J. C. Z. Woinarski, D. Driscoll, W. C. Dennison, and M. E. Jones. 2004. The role of connectivity in Australian conservation. Pacific Conservation Biology 10:266279.

Stacey, P. B., V. A. Johnson, and M. L. Taper. 1997. Migration within metapopulations: the impact upon local population dynamics. Pages 267-292 in I. Hanski, and M. E. Gilpin, editors. Metapopulation biology. Ecology, genetics and evolution. Academic Press, San Diego, CA.

Stokstad, E. 2009. On the Origin of Ecological Structure. Science 326:33-36.

Stone, L., and A. Roberts. 1990. The checkerboard score and species distributions. Oecologia-(Berlin) 85:74-79.

Strauss, S. Y. 1991. Indirect Effects in Community Ecology: Their Definition, Study and Importance. Trends in Ecology and Evolution 6:206-210.

Sundell, J., J. A. Eccard, and H. Ylönen. 2003. Predation rate, prey preference and predator switching: experiments on voles and weasels. Oikos 101:615-623.

Sündermann, D., M. Scheumann, and E. Zimmermann. 2008. Olfactory Predator Recognition in Predator-Naïve Gray Mouse Lemurs (Microcebus murinus). Journal of Comparative Psychology 122:146-155.

Swenson, N. G., B. J. Enquist, J. Pither, J. Thompson, and J. K. Zimmermann. 2006. The problem and promise of scale dependency in community phylogenetics. Ecology 87:2418-2424.

Swihart, R. K., Z. Feng, N. A. Slade, D. M. Mason, and T. M. Gehring. 2001. Effects of Habitat Destruction and Resource Supplementation in a Predator-Prey Metapopulation Model. Journal of theoretical Biology 210:287-303.

Swihart, R. K., T. M. Gehring, M. B. Kolozvary, and T. E. Nupp. 2003. Responses of 'resistant' vertebrates to habitat loss and fragmentation: the importance of niche breadth and range boundaries. Diversity and Distributions 9:1-18.

Swihart, R. K., J. R. Goheen, S. A. Schnelker, and C. E. Rizkalla. 2007. Testing the generality of patch and lanscape-level predictors of tree squirrel occurrence at a regional scale. Journal of Mammalogy 88:564-573.

Swihart, R. K., J. J. Lusk, J. E. Duchamp, C. E. Rizkalla, and J. E. Moore. 2006. The roles of landscape context, niche breadth, and range boundaries in predicting species responses to habitat alteration. Diversity and Distributions 12:277-287.

Swihart, R. K., and T. E. Nupp. 1998. Modeling population responses of North American tree squirrels to agriculturally induced fragmentation of forests. Pages 1-19 in M. A. Steel, J. F. Merritt, and D. A. Zegers, editors. Ecology and Evolutionary Biology of Tree Squirrels. Virginia Museum of Natural History.

Thomas, L., S. T. Buckland, E. A. Rexstad, J. L. Laake, S. Strindberg, S. L. Hedley, J. R. B. Bishop, A. Marques, and K. P. Burnham. 2010. Distance software: design and analysis of distance sampling surveys for estimating population size. Journal of Applied Ecology 47:5-14.

Thomas, L., J. L. Laake, E. Rexstad, S. Strindberg, F. F. C. Marques, S. T. Buckland, D. L. Borchers, D. R. Anderson, K. P. Burnham, M. L. Burt, S. L. Hedley, J. H. Pollard, J. R. B. Bishop, and T. A. Marques. 2009. Distance 6.0 Release 1 in Research-Unit-for-Wildlife-Population-Assessment, editor. University of St. Andrews, UK.

Thornton, D. H., M. E. Sunquist, and M. B. Main. 2004. Ecological separation within newly sympatric populations of coyotes and bobcats in south-central Florida. Journal of Mammalogy 85:973-982.

Tokeshi, M. 1999. Species Coexistence - Ecological and Evolutionary Perspectives. Blackwell Science Ltd, Oxford.

Umapathy, G., and A. Kumar. 2000. The occurrence of arboreal mammals in the rain forest fragments in the Anamalai Hills, south India. Biological-Conservation 92:311-319.

Vamosi, J. C., and S. M. Vamosi. 2007. Body size, rarity, and phylogenetic community structure: insights from diving beetle assemblages of Alberta. Diversity and Distributions 13:1-10.

Volterra. 1926. Variations and fluctuations of the numbers of individuals in animal species living together. in $\mathrm{R}$. N. Chapman, editor. Animal Ecology. McGraw Hill, New York.

Vos, C. C., J. Verboom, P. F. M. Opdam, and C. J. F. Ter Braak. 2001. Toward Ecologically Scaled Landscape Indices. The American Naturalist 183:24-41.

Wellborn, G. A. 2002. Trade-off between competitive ability and anti-predator adaptation in a freshwater amphipod species complex. Ecology 83:129-136. 
Wilbur, H. M. 1982. Competition between tadpoles of Hyla femoralis and Hyla gratiosa in Laboratory Experiments. Ecology 63:278-282.

Wilbur, H. M., and I. E. Fauth. 1990. Experimental aquatic food webs: interactions between two predators and two prey. American Naturalist 135:176-204.

Wimmer, B. 2000. Untersuchung der Paarungssysteme und der Populationsstruktur von Lemuren an Coquerels Zwergmausmaki (Mirza coquereli), dem grauen Mausmaki (Microcebus murinus), dem Rotstirnmaki (Eulemur fulvus rufus) und dem Larvensifaka (Propithecus verreauxi verreauxi). Page 131. Fakultät für Biologie. Ludwig Maximilians-Universität, München.

Wimmer, B., D. Tautz, and P. M. Kappeler. 2002. The genetic population structure of the gray mouse lemur (Microcebus murinus), a basal primate from Madagascar. Behav Ecol Sociobiol 52:166-175.

With, K. A., and A. W. King. 1999. Extinction Thresholds for Species in Fractal Landscapes. Conservation Biology 13:314-326.

Wolff, J. O. 1999. Behavioral Model Systems. Pages 11-29 in G. W. Barret, and J. D. Peles, editors. Landscape Ecology of Small Mammals. Springer, New York.

Ylönen, H., R. Pech, and S. Davis. 2003. Heterogeneous landscapes and the role of refuge on the population dynamics of a specialist predator and its prey. Evolutionary Ecology 17:349-369.

Yoder, A. D., R. M. Rasoloarison, S. M. Goodman, J. A. Irwin, S. Atsalis, M. J. Ravosa, and J. U. Ganzhorn. 2000. Remarkable species diversity in Malagasy mouse lemurs (primates, Microcebus). PNAS 97:1132511330.

Zapata, S. C., A. Travaini, M. Delibes, and R. Martinez-Peck. 2005. Food habits and resource partitioning between grey and culpeo foxes in southeastern Argentine Patagonia. Studies on Neotropical Fauna and Environment 40.

Zimmermann, E., E. Vorobieva, and D. Wrogemann. 2000. Use of Vocal Fingerprinting for Specific Discrimination of Gray (Microcebus murinus) and Rufous Mouse Lemurs (Microcebus rufus). International Journal of Primatology 21:837-852.

Zinner, D., and F. Torkler. 2005. Menabe Forest 2003. Forest classification, Landsat 7 ETM.

Ziv, Y., and B. P. Kotler. 2003. Giving-up densities of foraging gerbils: the effect of interspecific competition on patch use. Evolutionary Ecology 17:333-347.

Zollner, P. A. 2000. Comparing the landscape level perceptual abilities of forest sciurids in fragmented agricultural landscapes. Landscape Ecology 15:523-533. 


\section{APPENDIX}

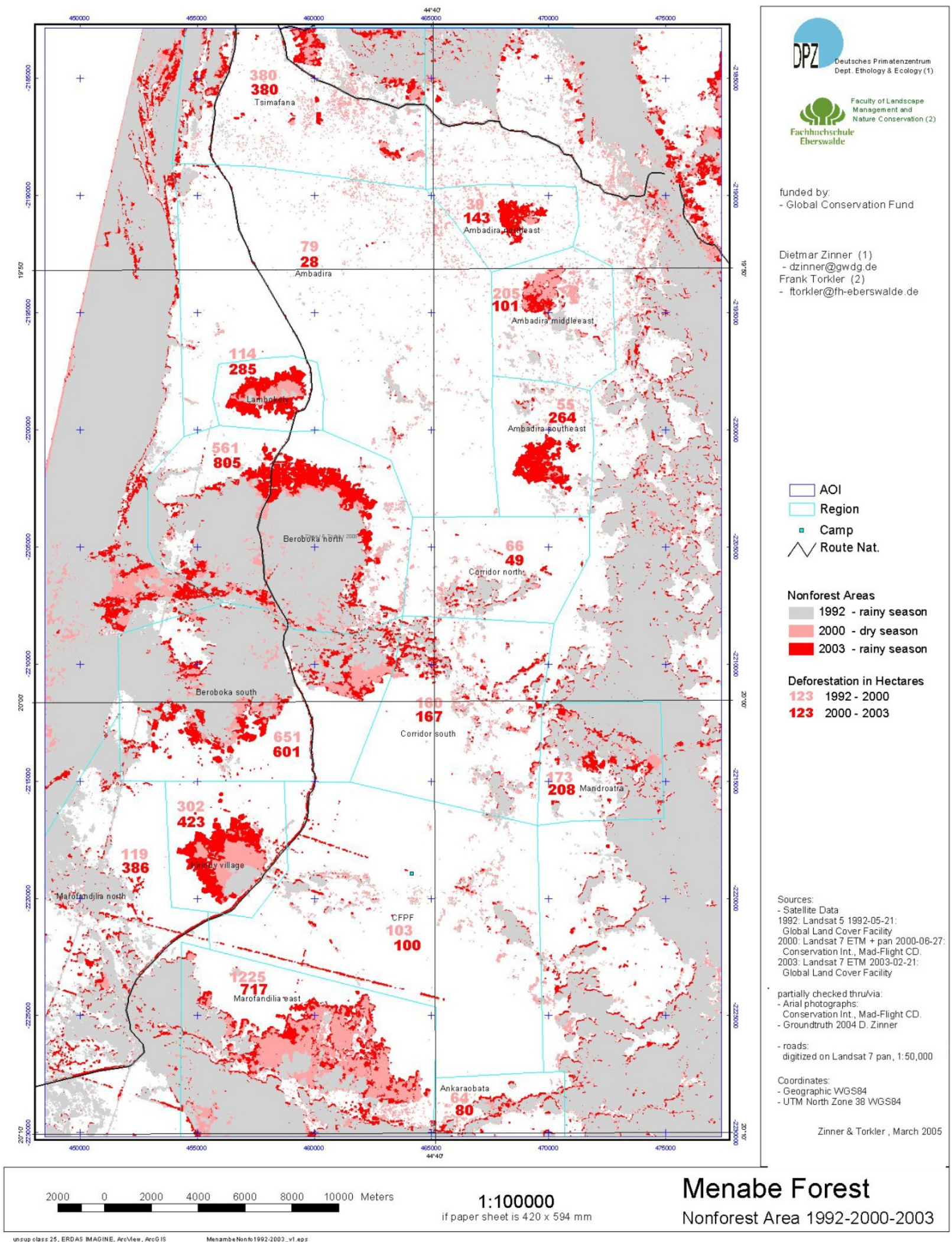

Fig. 74: Deforestation in Menabe Central from 1992-2003 (Zinner \& Torkler 2005) 


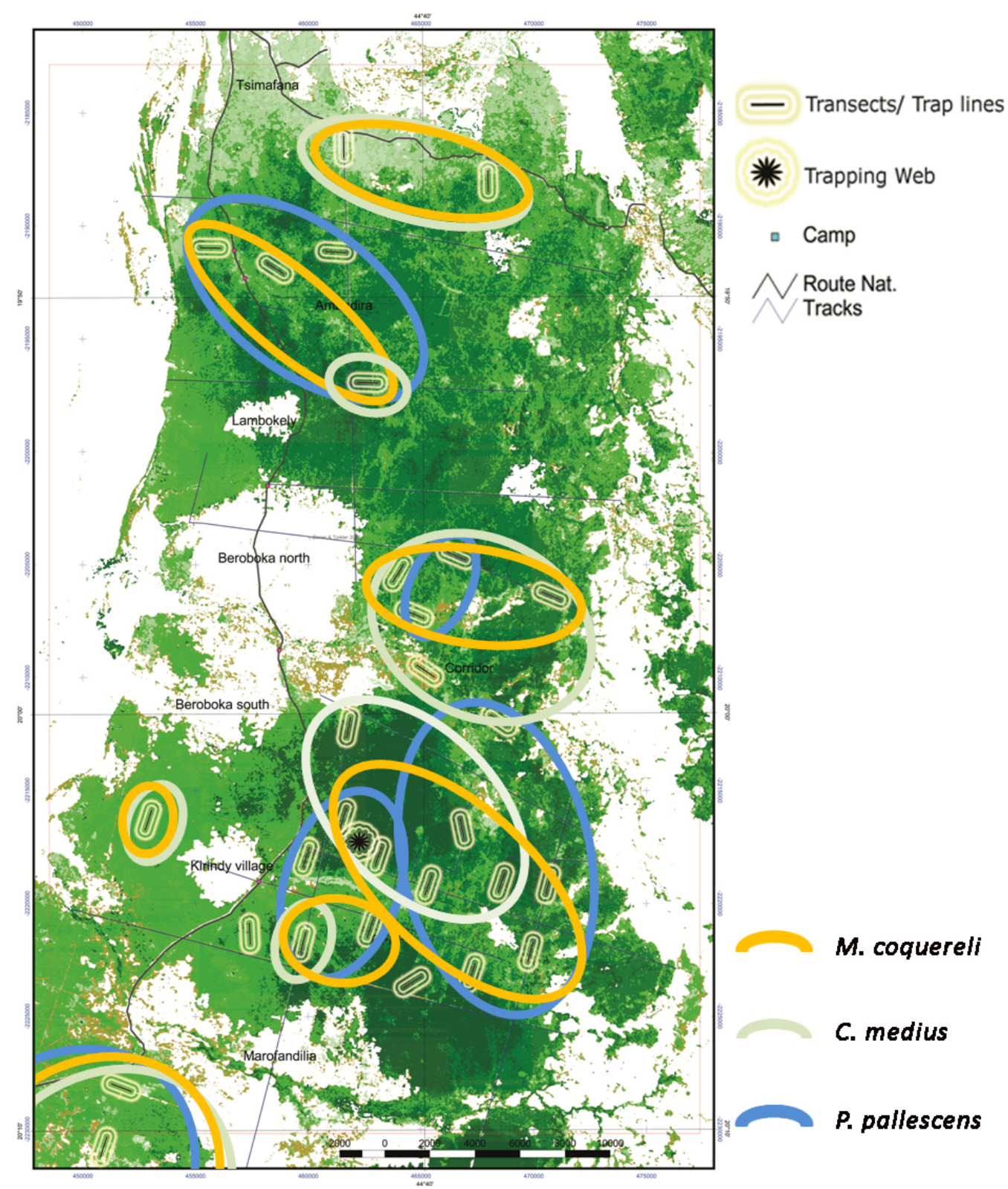

Fig. 75: regional distributions of $M$. coquereli (orange) and $C$. medius (light green), based on distance sampling and line trapping, and of $P$. pallescens (blue), based on distance sampling only. 
Table 13: variance analysis of $M$. murinus encounter rates per survey with transect category and area as explanatory variables (2-factorial ANOVA)

\begin{tabular}{|l|l|r|r|r|r|r|r|}
\hline year & season & $\mathbf{F}$ (arealD) & \multicolumn{1}{c|}{ df } & $\mathbf{p}$ (area ID) & $\mathbf{F}$ (transect.cat) & df & p (transect.cat) \\
\hline 2004 & DS & 2,177 & 1 & 0,174 & 0,171 & 1 & 0,689 \\
\hline 2006 & DS & 4,631 & 2 & $* 0,035$ & 0,123 & 2 & 0,886 \\
\hline 2007 & DS & 4,802 & 2 & $* 0,027$ & 1,191 & 2 & 0,335 \\
\hline $2007-1$ & RS & 0,277 & 1 & 0,613 & 0,261 & 2 & 0,776 \\
\hline $2007-2$ & RS & 0,660 & 3 & 0,590 & 0,233 & 2 & 0,795 \\
\hline
\end{tabular}

\begin{tabular}{|l|l|r|r|r|r|r|r|}
\hline year & season & $\mathbf{F}$ (interaction) & df & df_error & $\mathbf{p}$ (interaction) & R-square & $\begin{array}{c}\text { R-square } \\
\text { korr. }\end{array}$ \\
\hline 2004 & DS & 0,017 & 1 & 9 & 0,900 & 0,221 & $-0,039$ \\
\hline 2006 & DS & 2,110 & 2 & 11 & 0,168 & 0,602 & 0,385 \\
\hline 2007 & DS & 1,558 & 2 & 13 & 0,247 & 0,551 & 0,344 \\
\hline $2007-1$ & RS & 0,118 & 1 & 8 & 0,740 & 0,167 & $-0,249$ \\
\hline $2007-2$ & RS & 0,305 & 3 & 14 & 0,822 & 0,209 & $-0,243$ \\
\hline
\end{tabular}

Table 14: specific differences in encounter rates between repeated runs within single surveys

\begin{tabular}{|c|c|c|c|c|c|c|c|c|}
\hline \multirow[b]{2}{*}{ survey } & \multirow[b]{2}{*}{ df1 } & \multirow[b]{2}{*}{ df2 } & \multicolumn{3}{|c|}{ M. berthae } & \multicolumn{3}{|c|}{ M. murinus } \\
\hline & & & $\begin{array}{c}\text { Cronbachs } \\
\alpha\end{array}$ & $\begin{array}{c}\text { ANOVA } \\
F\end{array}$ & p & $\begin{array}{c}\text { Cronbachs } \\
\alpha\end{array}$ & $\begin{array}{c}\text { ANOVA } \\
\mathrm{F}\end{array}$ & p \\
\hline CFPF 2003 & 17 & 18 & 0.738 & 1.659 & 0.215 & 0.681 & 1.494 & 0.238 \\
\hline Kirindy 2004 & 8 & 9 & 0.423 & 1.220 & 0.302 & 0.697 & 0.029 & 0.868 \\
\hline N5+Corridor 2007 & 7 & 8 & 0.707 & 3.330 & 0.111 & 0.818 & 0.269 & 0.620 \\
\hline Kirindy 2007-1 & 10 & 11 & 0.971 & 0.000 & 1.000 & 0.964 & 0.957 & 0.351 \\
\hline Ambadira (all) & 6 & 7 & 0.962 & 1.000 & 0.356 & 0.335 & 0.300 & 0.604 \\
\hline
\end{tabular}

\begin{tabular}{|c|c|c|c|c|c|c|c|c|c|c|c|}
\hline \multirow[b]{2}{*}{ survey } & \multirow[b]{2}{*}{ df1 } & \multirow[b]{2}{*}{ df2 } & \multicolumn{3}{|c|}{ M. coquereli } & \multicolumn{3}{|c|}{ P. pallescens } & \multicolumn{3}{|c|}{ C. medius } \\
\hline & & & $\begin{array}{c}\text { Cronbachs } \\
\alpha\end{array}$ & $\begin{array}{c}\text { ANOVA } \\
F\end{array}$ & p & $\begin{array}{c}\text { Cronbachs } \\
\alpha\end{array}$ & $\begin{array}{c}\text { ANOVA } \\
F\end{array}$ & p & $\begin{array}{c}\text { Cronbachs } \\
\alpha\end{array}$ & $\begin{array}{c}\text { ANOVA } \\
F\end{array}$ & p \\
\hline CFPF 2003 & 17 & 18 & -0.055 & 1.572 & 0.227 & 0.595 & 0.777 & 0.390 & 0.688 & 0.387 & 0.548 \\
\hline $\begin{array}{l}\text { Kirindy } \\
2004\end{array}$ & 8 & 9 & 0.738 & 0.000 & 1.000 & 0.852 & 2.667 & 0.141 & 0.960 & 9.000 & 0.058 \\
\hline $\begin{array}{l}\text { N5+Corridor } \\
2007\end{array}$ & 7 & 8 & -0.516 & 1.340 & 0.285 & 0.857 & 0.000 & 1.000 & 0.351 & 0.286 & 0.621 \\
\hline $\begin{array}{l}\text { Kirindy } \\
2007-1\end{array}$ & 10 & 11 & 0.938 & 1.000 & 0.341 & 0.066 & 1.036 & 0.333 & & & \\
\hline $\begin{array}{l}\text { Ambadira } \\
\text { (all) }\end{array}$ & 6 & 7 & 0.918 & 0.300 & 0.604 & 0.596 & 0.632 & 0.457 & & & \\
\hline
\end{tabular}


Table 15: specific differences in encounter rates between repeated surveys within the same season

\begin{tabular}{|c|c|c|c|c|c|c|c|c|}
\hline \multirow[b]{2}{*}{ season } & \multirow[b]{2}{*}{ df1 } & \multirow[b]{2}{*}{ df2 } & \multicolumn{3}{|c|}{ M. berthae } & \multicolumn{3}{|c|}{ M. murinus } \\
\hline & & & $\begin{array}{c}\text { Cronbachs } \\
\alpha\end{array}$ & $\begin{array}{c}\text { ANOVA } \\
F\end{array}$ & p & $\begin{array}{c}\text { Cronbachs } \\
\alpha\end{array}$ & $\begin{array}{c}\text { ANOVA } \\
\mathrm{F}\end{array}$ & p \\
\hline dry season & 14 & 15 & 0.756 & 0.792 & 0.388 & 0.861 & 1.762 & 0.206 \\
\hline $\begin{array}{r}\text { rainy } \\
\text { season }\end{array}$ & 15 & 16 & 0.464 & 0.232 & 0.637 & 0.825 & 3.253 & 0.091 \\
\hline
\end{tabular}

\begin{tabular}{|c|c|c|c|c|c|c|c|c|c|c|c|}
\hline \multirow[b]{2}{*}{ season } & \multirow[b]{2}{*}{ df1 } & \multirow[b]{2}{*}{ df2 } & \multicolumn{3}{|c|}{ M. coquereli } & \multicolumn{3}{|c|}{ P. pallescens } & \multicolumn{3}{|c|}{ C. medius } \\
\hline & & & $\begin{array}{c}\text { Cronbachs } \\
\alpha \\
\end{array}$ & $\begin{array}{c}\text { ANOVA } \\
\mathrm{F}\end{array}$ & $p$ & $\begin{array}{c}\text { Cronbachs } \\
\alpha\end{array}$ & $\begin{array}{c}\text { ANOVA } \\
\mathrm{F}\end{array}$ & $p$ & $\begin{array}{c}\text { Cronbachs } \\
\alpha\end{array}$ & $\begin{array}{c}\text { ANOVA } \\
\mathrm{F}\end{array}$ & p \\
\hline dry season & 14 & 15 & 0.427 & 1.672 & 0.217 & 0.577 & 2.134 & 0.166 & & & \\
\hline $\begin{array}{r}\text { rainy } \\
\text { season }\end{array}$ & 15 & 16 & 0.833 & 0.024 & 0.879 & -0.049 & 0.033 & 0.858 & -0.168 & 0.828 & 0.382 \\
\hline
\end{tabular}

Table 16: RBD Kirindy DS 2006, DS 2007, RS 2007-1, RS 2007-2

\begin{tabular}{|l|r|r|r|r|r|r|}
\hline species & F(year) & df(year) & p(year) & F(season) & df(season) & p(season) \\
\hline M. berthae & 1.426 & 1 & 0.244 & 0.000 & 1 & 1.000 \\
\hline M. murinus & 0.387 & 1 & 0.540 & 1.189 & 1 & 0.286 \\
\hline M. coquereli & 0.045 & 1 & 0.834 & 16.164 & 1 & 0.001 \\
\hline P. pallescens & 0.003 & 1 & 0.956 & 1.680 & 1 & 0.207 \\
\hline
\end{tabular}

\begin{tabular}{|l|r|r|r|r|r|r|}
\hline species & F(interaction) & df(interaction) & $\mathbf{p}$ (interaction) & df_error & $\mathbf{R}^{\mathbf{2}}$ & $\mathbf{R}^{\mathbf{2}}$ corr. \\
\hline M. berthae & 0.634 & 1 & 0.434 & 24 & 0.079 & -0.036 \\
\hline M. murinus & 0.000 & 1 & 0.992 & 24 & 0.062 & -0.056 \\
\hline M. coquereli & 0.045 & 1 & 0.834 & 24 & 0.404 & -0.329 \\
\hline P. pallescens & 0.003 & 1 & 0.956 & 24 & 0.066 & -0.051 \\
\hline
\end{tabular}

Table 17: differences in encounter rates between early (RS1) and late (RS2) rainy season (Wilcoxon)

\begin{tabular}{|l|l|l|r|}
\hline \multicolumn{1}{|c|}{ species } & Z & df & p (2-sided) \\
\hline ER_Mb.rs2 - ER_Mb.rs1 & $-0.282^{\mathrm{a}}$ & 15 & 0.778 \\
\hline ER_Mm.rs2 - ER_Mm.rs1 & $-1.649^{\mathrm{a}}$ & 15 & 0.099 \\
\hline ER_Cm.rs2 - ER_Cm.rs1 & $-0.747^{\mathrm{b}}$ & 11 & 0.455 \\
\hline ER_Mc.rs2 - ER_Mc.rs1 & $-0.213^{\mathrm{a}}$ & 15 & 0.832 \\
\hline ER_Pp.rs2 - ER_Pp.rs1 & $-0.313^{\mathrm{b}}$ & 16 & 0.754 \\
\hline
\end{tabular}

\footnotetext{
${ }^{a}$ based on positive ranks
}

${ }^{b}$ based on negative ranks 
Table 18: DISTANCE population density estimates per stratum (survey unit)

\begin{tabular}{|c|c|c|c|c|c|c|c|c|c|c|c|c|}
\hline \multirow[b]{2}{*}{ area } & \multirow[b]{2}{*}{ year } & \multirow[b]{2}{*}{ season } & \multicolumn{2}{|c|}{ M. berthae } & \multicolumn{2}{|c|}{ M. murinus } & \multicolumn{2}{|c|}{ M. coquereli } & \multicolumn{2}{|c|}{ C. medius } & \multicolumn{2}{|c|}{ P. pallescens } \\
\hline & & & $\begin{array}{c}\text { ind./ } \\
\text { km }^{2}\end{array}$ & $\% \mathrm{CV}$ & $\begin{array}{l}\text { ind.// } \\
\mathrm{km}^{2}\end{array}$ & $\% \mathrm{CV}$ & $\begin{array}{l}\text { ind./ } \\
\mathrm{km}^{2}\end{array}$ & $\% \mathrm{CV}$ & $\begin{array}{l}\text { ind./ } \\
\text { km }^{2}\end{array}$ & $\% \mathrm{CV}$ & $\begin{array}{l}\text { ind./ } \\
\mathrm{km}^{2}\end{array}$ & $\% \mathrm{CV}$ \\
\hline \multirow{4}{*}{ 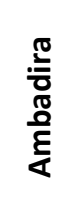 } & 2004 & DS & 51.58 & 59.15 & 69.391 & 28.97 & 33.14 & 58.25 & & & 36.426 & 29.4 \\
\hline & 2006 & DS & 137.53 & 100.82 & 0 & & 198.84 & 34.22 & & & 24.284 & 100.16 \\
\hline & 2007-1 & RS & 283.66 & 100.82 & 0 & & 115.99 & 100.3 & 96.916 & 14.97 & 18.213 & 100.16 \\
\hline & 2008 & RS & 77.36 & 44.82 & 69.391 & 45.71 & 157.42 & 27.44 & 83.071 & 49.27 & 0 & \\
\hline & & mean & 137.53 & & 34.70 & & 126.35 & & 89.99 & & 19.73 & \\
\hline
\end{tabular}

\begin{tabular}{|c|c|c|c|c|c|c|c|c|c|c|c|c|}
\hline & & & M. ber & thae & M. $m \iota$ & rinus & M. co & yuereli & C. $m e$ & dius & P. pall & scens \\
\hline area & year & season & $\begin{array}{r}\text { ind./ } \\
\mathbf{k m}^{2} \\
\end{array}$ & $\% \mathrm{CV}$ & $\begin{array}{c}\text { ind./ } \\
\text { km }^{2}\end{array}$ & $\% \mathrm{CV}$ & $\begin{array}{l}\text { ind./ } \\
\mathrm{km}^{2}\end{array}$ & $\% \mathrm{CV}$ & $\begin{array}{l}\text { ind./ } \\
\mathbf{k m}^{2}\end{array}$ & $\% \mathrm{CV}$ & $\begin{array}{c}\text { ind./ } \\
\text { km }^{2}\end{array}$ & $\% \mathrm{CV}$ \\
\hline \multirow{6}{*}{ 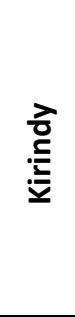 } & 2003 & DS & 103.84 & 42.34 & 251.49 & 17.94 & 13.345 & 53.79 & & & 44.005 & 30.44 \\
\hline & 2004 & DS & 59.72 & 54.59 & 284.87 & 26.73 & 34.884 & 50.38 & & & 72.852 & 49.58 \\
\hline & 2006 & DS & 46.89 & 66.58 & 145.09 & 53.24 & 6.0255 & 105.69 & & & 9.9344 & 54.72 \\
\hline & 2007 & DS & 8.60 & 105.67 & 180.42 & 28.76 & 13.256 & 71.13 & & & 10.928 & 54.29 \\
\hline & 2007-1 & RS & 68.77 & 61.57 & 343.49 & 37.49 & 15.781 & 82.96 & 168.91 & 21.9 & 24.284 & 59.69 \\
\hline & $2007-2$ & RS & 45.84 & 55.99 & 192.75 & 35.97 & 14.729 & 66.6 & 190.76 & 28.2 & 32.379 & 51.46 \\
\hline & & $n$ & 55.61 & & 233.02 & & 16.34 & & 179.84 & & 32.40 & \\
\hline
\end{tabular}

\begin{tabular}{|c|c|c|c|c|c|c|c|c|c|c|c|c|}
\hline \multirow{2}{*}{ area } & \multirow[b]{2}{*}{ year } & \multirow[b]{2}{*}{ season } & \multicolumn{2}{|c|}{ M. berthae } & \multicolumn{2}{|c|}{ M. murinus } & \multicolumn{2}{|c|}{ M. coquereli } & \multicolumn{2}{|c|}{ C. medius } & \multicolumn{2}{|c|}{ P. pallescens } \\
\hline & & & $\begin{array}{c}\text { ind./ } \\
\text { km }^{2}\end{array}$ & $\% \mathrm{CV}$ & $\begin{array}{r}\text { ind.// } \\
\mathrm{km}^{2}\end{array}$ & $\% \mathrm{CV}$ & $\begin{array}{l}\text { ind./ } \\
\text { km }^{2}\end{array}$ & $\% \mathrm{CV}$ & $\begin{array}{l}\text { ind./ } \\
\text { km }^{2}\end{array}$ & $\% \mathrm{CV}$ & $\begin{array}{c}\text { ind./ } \\
\text { km }^{2}\end{array}$ & $\% \mathrm{CV}$ \\
\hline \multirow{2}{*}{ 흔 흠 } & 2007 & DS & 68.77 & 61.57 & 289.13 & 32.83 & 22.093 & 50.60 & & & 33.391 & 51.89 \\
\hline & $2007-2$ & RS & 34.38 & 100.82 & 242.87 & 33.69 & 22.093 & 63.72 & 258.44 & 41.52 & 54.639 & 64.07 \\
\hline
\end{tabular}

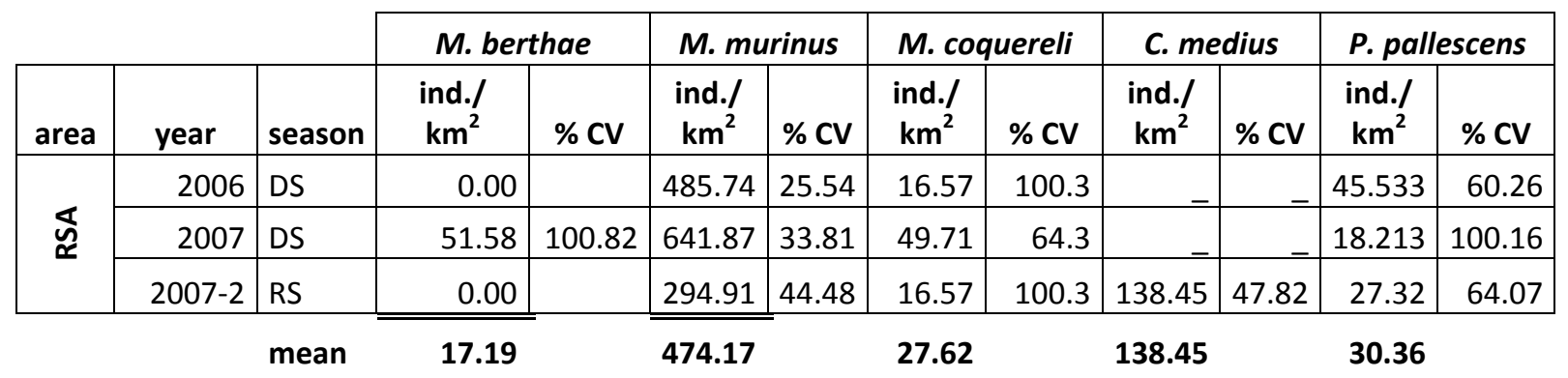




\section{Eidesstattliche Erklärung}

Ich gebe hiermit die Erklärung ab, dass ich die Dissertation mit dem Titel:

Determinants of population structure in the world's smallest primate, Microcebus berthae, across its global range in Menabe Central, Western Madagascar

am Deutschen Primatenzentrum, Abt. Für Verhaltensökologie und Soziobiologie/ Anthropologie

im Rahmen des PhD-Programms „Biodiversität und Ökologie“ am GCBE der Georg-August Universität Göttingen

unter Anleitung von Prof. P. M. Kappeler

1. selbstständig verfasst,

2. nur unter Benutzung der im Literaturverzeichnis angegebenen Arbeit angefertigt und sonst kein anderes gedrucktes oder ungedrucktes Material verwendet,

3. keine unerlaubte fremde Hilfe in Anspruch genommen,

4. $\quad$ sie weder in der gegenwärtigen noch in einer anderen Fassung einer in- oder ausländischen Fakultät als Dissertation, Semesterarbeit, Prüfungsarbeit, oder zur Erlangung eines akademischen Grades, vorgelegt habe.

Livia Schäffler 